\author{
Tesis de Doctorado
}

\title{
RADIACIÓN NO-TÉRMICA ASOCIADA A ONDAS DE CHOQUE ASTROFÍSICAS
}

\author{
Lic. Anabella T. Araudo \\ Director: Prof. Dr. Gustavo E. Romero
}

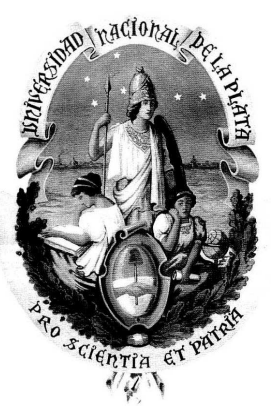

Universidad Nacional de La Plata

Facultad de Ciencias Astronómicas y Geofísicas

20 DE SEPTIEMBRE DE 2010 
Dedico esta tesis a Valentí

y a mis padres,

Susana y Arsenio. 


\section{Prefacio}

Los trabajos que, junto con mi director, presentaremos a continuación se engloban dentro de la tesis de doctorado titulada "Emisión no-térmica asociada a ondas de choque astrofísicas". La misma no podría haberse llevado a cabo sin la ayuda de muchas personas y el apoyo de algunas instituciones. Es por esto que, a modo personal, quisiera hacerles a ellas un sencillo, pero muy sincero, agradecimiento a través de las siguientes líneas.

En primer lugar quisiera agradecer a mi director, el Prof. Gustavo E. Romero, por haberme guiado en cada trabajo que hemos realizado. Le agradezco el haber compartido sus ideas conmigo, las discusiones que hemos tenido para poder implementarlas y por sobre todo sus consejos. Me enseñó a trabajar procurando un entendimiento global de los temas e inculcandomé un profundo interes ético en cada problema que hemos encarado. La siguiente frase ilustra como sus consejos me han servido para realizar la tesis, aprendiendo a salvar los inconvenientes del camino: "La casa queda lejos de aquí, pero usted no se perderá si toma ese camino a la izquierda y en cada encrucijada del camino dobla a la izquierda.'1

A los profesores Ana María Platzeck y Osvaldo Civitarese y al Dr. Felix Mirabel, todos ellos integrantes del jurado examinador de esta tesis, les agradezco los fructíferos comentarios realizados. En particular, quisiera agradecer a la Prof. Platzeck por la lectura minusciosa de cada capítulo (y por sus excelentes clases).

Al Consejo Nacional de Investigaciones Científicas y Técnicas (CONICET) le agradezco el haberme otorgado dos becas para realizar mis estudios e investigaciones doctorales.

Al Instituto Argentino de Radioastronomía (IAR) y a la Facultad de Ciencias Astronómicas y Geofísicas (FCAG) de la UNLP quisiera agradecerles el haberme facilitado las condiciones y los insumos necesarios para realizar los trabajos que forman parte de esta tesis.

I want to acknowledge the hospitality and suport of Prof. Felix Aharonian. He hosted me during three stays in the Max Plank Institut für Kernphysik in Heidelberg.

Al Prof. Josep María Paredes, le agradezco haberme invitado a exponer mis trabajos

\footnotetext{
${ }^{1}$ J.L. Borges, Extracto del cuento "El jardín de los senderos que se bifurcan", Ficciones.
} 
en la Facultat de Física de la Universitat de Barcelona.

A los integrantes del grupo de Astrofísica Relativista y Radioastronomía (GARRA) quisiera agradecerles el haberme permitido desarrollar la tesis en un ambiente cordial de trabajo. En particular, quisiera agradecer el apoyo brindado por Paula Benaglia e Ileana Andruchow.

A Federico Bareilles le agradezco la ayuda brindada para salvar diferentes (y muchos) problemas informáticos.

A todos mis amigos. En particular, voy a destacar a algunas personas muy importantes: Ceci Fariña, Vero Firpo, Andrea Fortier, Anahí Granada, Ielca Martinic, Antonella Monachesi y Claudia Scóccola. Con ellas he compartido gran parte de la carrera en la FCAG, además de charlas hermosas y discusiones de toda índole. A Javier Vasquez, a Nicolás Duronea y a Gonzalo De Elía, un agradecimiento especial por el compañerismo. A Pol Bordas, la alegría.

El agradecimiento más profundo y eterno es para mi familia. Ellos han sido (y serán siempre) el sustento emocional y la razón por la cual he podido realizar muchas acciones. A mis sobrinos (Bianca, Ernestina, Román e Iván) les quiero agradecer que desde que están en este mundo han llenado mi vida de ternura.

L'agraïment més especial de tots és per a una persona extraordinària, en Valentí BoschRamon. Com a col-laborador, el Dr. Bosch-Ramon $\mathrm{m}^{\prime}$ ha ajudat en cadascun dels treballs que hem realitzat plegats (i també en aquells en què ell no hi participava directament!). Amb ell he après molta astrofísica, $\mathrm{m}^{\prime}$ ha assistit fins al més mínim detall de cada article, i $\mathrm{m}^{\prime}$ ha aconsellat sobre com desenvolupar-me en diverses situacions. Però més enllà de tot això, gràcies Valentí per fer-me part de la teva vida. Gràcies per donar-me tant d'amor i fer de la vida quotidiana una bellíssima obra d’art. Les següents línies són d’una cançó que coneixes bé, i m'ajuden per a dir-te per que et necessito al meu costat:

Para decidir si sigo poniendo esta sangre en tierra, Este corazón que va de su parte, sol y tinieblas Para continuar caminando al sol por estos desiertos Para recalcar que estoy viva en medio de tantos muertos. Para decidir, para continuar, para recalcar y considerar Sólo me hace falta que estés aquí..2

\footnotetext{
${ }^{2}$ Razón de vivir, Victor Heredia.
} 
Finalmente, con la ayuda de todas las personas que mencioné antes, he encontrado la casa que quedaba tan lejos. Aludiendo una vez más a J.L. Borges, los invito a leer la tesis.

"Alguna vez, los senderos de ese laberinto convergen: por ejemplo, usted llega a esta casa, pero en uno de los pasados posibles usted es mi enemigo, en otro mi amigo. Si se resigna usted a mi pronunciación incurable, leeremos unas páginas. '33

Anabella Araudo

${ }^{3}$ J.L. Borges, Extracto del cuento "El jardín de los senderos que se bifurcan", Ficciones. 


\section{Índice general}

Resumen 3

Abstract 6

1. Introducción general 9

I Procesos no térmicos

2. Ondas de choque y aceleración de partículas 15

2.1. Introducción . . . . . . . . . . . . . . . . . . . . . . 15

2.2. Definición y estructura de las ondas de choque . . . . . . . . . . . 17

2.3. Ondas de choque no relativistas . . . . . . . . . . . . . 20 20

2.3.1. Ondas de choque hidrodinámicas . . . . . . . . . . . . 20

2.3.2. Ondas de choque magnetohidrodinámicas . . . . . . . . . . 25

2.4. Ondas de choque relativistas . . . . . . . . . . . . . . . . 26

2.5. Producción de ondas de choque . . . . . . . . . . . . . 27

2.5.1. Ondas de choque por perturbaciones locales . . . . . . . . 28

2.5.2. Ondas de choque por interacciones globales . . . . . . . . . . 30

2.6. Aceleración de partículas en choques . . . . . . . . . . . . . 32

2.6.1. Población de partículas no térmicas . . . . . . . . . . . 36

3. Procesos radiativos 39

3.1. Conceptos básicos . . . . . . . . . . . . . . . . . . . . . . 39

3.2. Procesos radiativos . . . . . . . . . . . . . . . . . . 41

3.2.1. Radiación sincrotrón . . . . . . . . . . . . . . . . . 41

3.2.2. Radiación Compton inversa . . . . . . . . . . . . . 43

3.2.3. Bremsstrahlung relativista . . . . . . . . . . . . . 46

3.2.4. Interacciones protón-protón . . . . . . . . . . . . 47

3.3. Absorción . . . . . . . . . . . . . . . . . . . . . . . . . 49

3.3.1. Absorción por creación de pares electrón-positrón ........ 50 


\section{Estudios de fuentes a diferentes escalas}

4. Objetos estelares jóvenes 55

4.1. Introducción . . . . . . . . . . . . . . . . . . . . . 55

4.2. La fuente IRAS $16547-4247$. . . . . . . . . . . . . . . . . . . . . 59

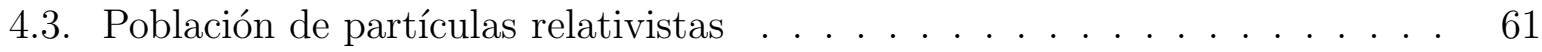

4.3.1. Choques terminales . . . . . . . . . . . . . . 62

4.3.2. Aceleración de partículas y pérdidas radiativas . . . . . . . . . 63

4.4. Distribuciones espectrales de energía . . . . . . . . . . . . . 70

4.4.1. Interacciones leptónicas . . . . . . . . . . . . . . . . . . . . . 70

4.4.2. Interacciones hadrónicas . . . . . . . . . . . . . . . . 70

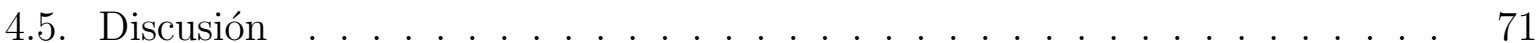

5. Microcuasares $\quad 75$

5.1. Introducción . . . . . . . . . . . . . . . . . . 75

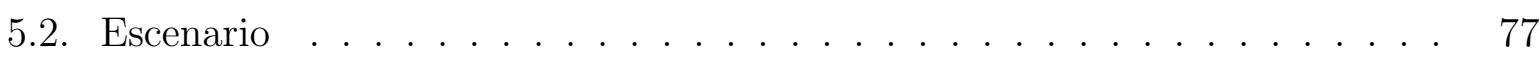

5.2.1. Interacción jet-clump . . . . . . . . . . . . . . . . 79

5.2.2. Emisión térmica del clump ................. 81

5.3. Población de partículas relativistas . . . . . . . . . . . . . . . 83

5.3.1. Aceleración de partículas y pérdidas radiativas . . . . . . . . . . 83

5.4. Distribuciónes espectrales de energía . . . . . . . . . . . . . 87

5.4.1. Emisión asociada al bow shock . . . . . . . . . . . . . 87

5.4.2. Emisión asociada al clump . . . . . . . . . . . . . . 88

5.5. Interacciones simultáneas . . . . . . . . . . . . . . . . . . . . . . 89

5.6. Discusión ........................ . . . . . . . . . . . .

6. Núcleos de galaxias activas

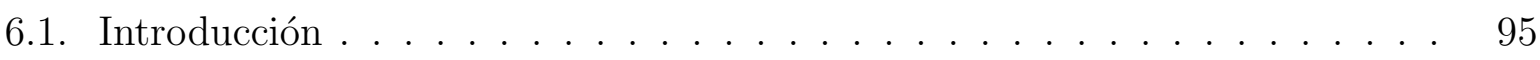

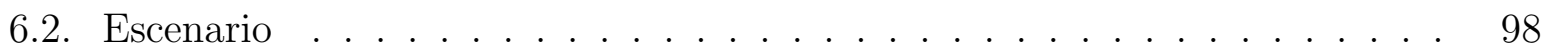

6.2.1. Interacción jet-nube . . . . . . . . . . . . . . . . 99

6.3. Población de partículas relativistas . . . . . . . . . . . . . . . . 103

6.3.1. Aceleración de partículas y pérdidas radiativas . . . . . . . . . 105

6.4. Distribuciones espectrales de energía . . . . . . . . . . . . . . . . . 109

6.4.1. Emisión asociada al bow shock . . . . . . . . . . . . . . . . . . 109

6.4.2. Emisión asociada a la nube . . . . . . . . . . . . . . . 110

6.5. Interacciones múltiples . . . . . . . . . . . . . . . . . . . . . . . . 110

6.6. Aplicaciones . . . . . . . . . . . . . . . . . 112

6.6.1. Galaxias FR I: Cen A . . . . . . . . . . . . . . . 112

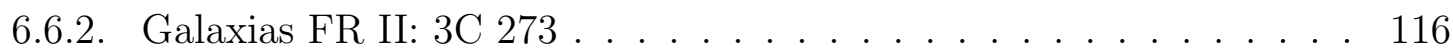

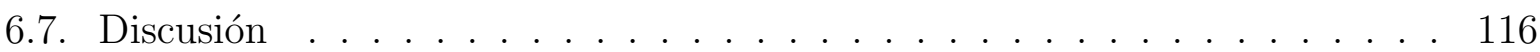


7. Cúmulos de galaxias $\quad \mathbf{1 1 9}$

7.1. Introducción . . . . . . . . . . . . . . . . . . . . . . . 119

7.2. El cúmulo Abell 3376 . . . . . . . . . . . . . . . . . . . . . . . 121

7.3. Población de partículas relativistas . . . . . . . . . . . . . . 125

7.3.1. Aceleración de partículas y pérdidas radiativas . . . . . . . . . . . 126

7.4. Distribuciones espectrales de energía . . . . . . . . . . . . . . . . 131

7.4.1. Interacciones leptónicas . . . . . . . . . . . . . . . 132

7.4.2. Interacciones hadrónicas . . . . . . . . . . . . . . . . 132

7.5. Discusión . . . . . . . . . . . . . . . . . . . . . . 134

8. Conclusiones y perspectivas 135

Referencias 140

A. Lista de acrónimos 147

B. Lista de publicaciones 149 



\section{Resumen}

El objetivo principal de esta tesis es investigar los procesos físicos que dan lugar a emisión no-térmica a altas energías en objetos astrofísicos capaces de acelerar partículas hasta velocidades relativistas. En particular, se ha estudiado la emisión de rayos gamma producida en fuentes cósmicas con diferentes escalas espaciales, desde objetos estelares jóvenes hasta cúmulos de galaxias, pasando por microcuasares y núcleos de galaxias activas. En los dos primeros tipos de objetos se ha modelado la emisión de rayos gamma a partir de los datos obtenidos en frecuencias radio de las fuentes IRAS 16547-4247 y Abell 3376. En los dos últimos, se ha desarrollado un modelo específico de emisión basado en la interacción de inhomogeneidades del medio externo con los jets producidos por el objeto compacto. Específicamente, se han considerado clumps o grumos del viento de la estrella compañera en los microcuasares y nubes de la región de formación de líneas anchas en las galaxias activas, interactuando con los jets de las fuentes. En todos los casos, los modelos desarrollados permiten realizar predicciones contrastables por la nueva generación de instrumentos que operan en altas energías, tales como los satélites Fermi y AGILE y los telescopios Cherenkov HESS, MAGIC y el planeado CTA.

Palabras claves:

- Rayos gamma: general

- Ondas de choque: general

- Ondas de choque: aceleración de partículas

- Procesos radiativos: no térmicos

- Estrellas de gran masa: formación: emisión no térmica

- Estrellas de gran masa: vientos

- Sistemas binarios: microcuasares: general

- Galaxias activas: general

- Cúmulos de galaxias: Abell 3376 



\section{Abstract}

The main goal of this thesis is to study the physical processes that can produce nonthermal emission at high energies in astrophysical objects capable to accelerate particles up to relativistic velocities. In particular, we have studied the gamma-ray emission produced in cosmic sources with different spatial scales, from young stellar objects to clusters of galaxies, going through microquasars and active galactic nuclei. In the former cases, we have modeled the gamma-ray emission using the radio data from the sources IRAS 165474247 and Abell 3376. In the latter, we have developed a specific radiation model based on the interaction of the inhomogeneities of the external medium with the jets generated by the compact object. Specifically, we have considered clumps of the massive stellar wind in microquasars, and clouds of the broad line region in active galactic nuclei, interacting with the jets of the sources. In all cases, the developed models allow us to make predictions testables with the new generation of instruments operating at high energies, such as the satellites Fermi and AGILE, and the Cherenkov telescopes HESS, MAGIC, and the forthcoming CTA.

Key words:

- Gamma-rays: general

- Shock waves: general

- Shock waves: particle acceleration

- Radiative processes: non-thermal

- Massive stars: formation: non-thermal emission

- Massive stars: winds

- Binary systems: microquasars: general

- Active galaxies: general

- Clusters of galaxies: Abell 3376 


\section{Capítulo 1}

\section{Introducción general}

La astronomía es la ciencia que estudia los fenómenos que ocurren fuera de nuestro planeta. La tecnología actual ha permitido estudiar in situ los planetas y satélites naturales cercanos, accediendo a ellos a través de sondas y naves tripuladas. Sin embargo, para los procesos que ocurren fuera del sistema solar, la astronomía aún sigue investigando casi como en la antiguedad, es decir, a través de la luz que llega a nuestros detectores desde los objetos celestes. Esta radiación, en cada banda de frecuencia, nos ofrece una fenomenología distinta, ya que los procesos físicos subyacentes pueden ser muy variados. En particular, la emisión de rayos gamma da cuenta de los procesos no térmicos, es decir, fuera del equilibrio termodinámico, que puedan tener lugar en la fuente. El Universo en rayos gamma es puramente no térmico, ya que la temperatura requerida para emitir fotones gamma térmicamente es extremadamente alta $\left(\sim 10^{13} \mathrm{~K}\right)$ y difícilmente puedan tenerla los sistemas físicos conocidoł1.

Los rayos gamma forman la última banda del espectro electromagnético, abarcando más de 14 órdenes de magnitud en energía:

$$
5 \times 10^{5} \mathrm{eV} \lesssim E_{\gamma} \lesssim 10^{20} \mathrm{eV}
$$

El límite inferior, $E_{\gamma} \sim m_{e} c^{2} \sim 5 \times 10^{5} \mathrm{eV}$, corresponde a la emisión de líneas, como la de aniquilación de los pares electrón-positrón $e^{ \pm}$, mientras que el valor superior, $E_{\gamma} \sim$ $10^{20} \mathrm{eV}$, corresponde a los rayos cósmicos más energéticos que han sido detectados. Dada la gran amplitud de esta banda de energía, resulta conveniente subdividirla en las siguientes regiones:

- $E_{\gamma}<30 \mathrm{MeV}$ : Baja energía (LE, por Low Energy).

- $30 \mathrm{MeV}<E_{\gamma}<30 \mathrm{GeV}$ : Alta energía (HE, por High Energy).

- $30 \mathrm{GeV}<E_{\gamma}<30 \mathrm{TeV}:$ Muy alta energía (VHE, por Very High Energy).

\footnotetext{
${ }^{1}$ Temperaturas $\sim 10^{13} \mathrm{~K}$ podrían alcanzarse por períodos de tiempo cortos en eventos explosivos muy energéticos como el Big Bang o los eruptores de rayos gamma (GRBs, por Gamma Ray Bursts).

${ }^{2} 1 \mathrm{TeV}=10^{12} \mathrm{eV}, 1 \mathrm{PeV}=10^{15} \mathrm{eV}$ y $1 \mathrm{EeV}=10^{18} \mathrm{eV}$
} 
- $30 \mathrm{TeV}<E_{\gamma}<30 \mathrm{PeV}$ : Ultra alta energía (UHE, por Ultra High Energy).

- $E_{\gamma}>30 \mathrm{PeV}$ : Extremadamente alta energía (EHE, por Extremely High Energy).

La observación en las bandas LE y HE debe realizarse desde satélites espaciales, ya que la atmósfera terrestre es opaca para las frecuencias correspondientes. Por otro lado, los rayos gamma de energías $\gtrsim 30 \mathrm{GeV}$ pueden detectarse indirectamente desde la superficie de la Tierra mediante telescopios Cherenkov. Los rayos gamma, al penetrar en la atmósfera interactúan con los campos allí presentes (magnéticos, de materia y de fotones) produciendo pares $e^{ \pm}$. Estos leptones, interactuando con los mismos campos, pueden crear más fotones y pares menos energéticos, desarrollando así una cascada electromagnética. La luz (visible) Cherenkov producida por los leptones relativistas en la atmósfera puede detectarse desde la superficie terrestre. Reconstruyendo la cascada se puede determinar la energía del fotón gamma original y la dirección de arribo, que nos indica la localización de la fuente. Sin embargo, sólo hay detección de fuentes hasta la región de las UHE y por lo tanto solo para $E_{\gamma}<\mathrm{PeV}$ puede hablarse de una astronomía de rayos gamma.

Los telescopios de rayos gamma que actualmente están en funcionamiento son los siguientes:

- Telescopios espaciales $\left(E_{\gamma} \lesssim 30 \mathrm{GeV}\right)$

- Fermi: Es el instrumento más nuevo que se ha puesto en funcionamiento. Fue lanzado por la NASA en el año 2008 y puede detectar fotones con energías $20 \mathrm{MeV} \lesssim E_{\gamma} \lesssim 100 \mathrm{GeV}$.

- AGILE: Es un satélite italiano que funciona en el rango de energías $0.1 \mathrm{GeV}$ $\lesssim E_{\gamma} \lesssim 30 \mathrm{GeV}$.

- Swift: Lanzado por la NASA en el año 2004, puede detectar fotones gamma con energías de $\sim 150 \mathrm{keV}$. Además del instrumento BAT de rayos X duros (pero que llega a detectar algunos fotones gamma blandos), a bordo del satélite hay un telescopio ultravioleta (UV) y otro óptico, con el fin de poder detectar GRBs en diferentes longitudes de onda.

- Telescopios Cherenkov $\left(E_{\gamma} \gtrsim 30 \mathrm{GeV}\right)$

- HESS: Es un arreglo de 4 telescopios franco-alemanes de $12 \mathrm{~m}$ de diámetro cada uno ubicados en Gamsberg, Namibia. Opera en el rango de energías $50 \mathrm{GeV}$ $\lesssim E_{\gamma} \lesssim 10 \mathrm{TeV}$

- MAGIC: Es un único receptor germano-español de $17 \mathrm{~m}$ de diámetro ubicado en La Palma y detecta fotones gamma de energías $50 \mathrm{GeV} \lesssim E_{\gamma} \lesssim 10 \mathrm{TeV}$.

- VERITAS: Es un arreglo de 4 telescopios estadounidenses de $12 \mathrm{~m}$ de diámetro cada uno, ubicados en Arizona, y que opera en la banda de energías $100 \mathrm{GeV}$ $\lesssim E_{\gamma} \lesssim 50 \mathrm{TeV}$. 
- Cangaroo III: Es una colaboración japonesa-australiana que opera un arreglo de 4 telescopios de $10 \mathrm{~m}$ cada uno ubicados en Woomera, en el sur de Australia. Detecta rayos gamma de $E_{\gamma} \sim 100 \mathrm{GeV}$.

Todos estos instrumentos detectan fuentes, y de ahí que los llamemos telescopios. Estas fuentes pueden ser puntuales o extendidas, como por ejemplo la región central de la Galaxia. Entre las fuentes detectadas por los telescopios listados anteriormente, hay núcleos de galaxias activas (AGN, por Active Galactic Nuclei), pulsares, remanentes de supernovas, binarias de rayos X, GRBs y algunas regiones de formación estelar. Hay otras fuentes que han sido observadas, pero cuya detección 3 no ha sido clamada aún como ocurre con los cúmulos de galaxias y algunas regiones de formación estelar. Por otro lado, hay muchas fuentes que aún no han sido identificadas. En la Figura 1.1 se muestra un mapa del cielo con las fuentes detectadas en el rango de las VHE por diferentes telescopios.

La detección de fuentes gamma es importante porque nos provee información sobre procesos físicos extremos. La astrofísica de rayos gamma es una disciplina única para estudiar los fenómenos fuera del equilibrio que involucran las enormes cantidades de energía requeridas para emitir los fotones más energéticos del espectro electromagnético. Así, los emisores de rayos gamma deben ser regiones del espacio en las cuales las partículas relativistas allí presentes pueden enfriarse eficientemente debido a interacciones con grandes densidades de materia y/o radiación.

En esta tesis nos hemos propuesto estudiar los procesos no térmicos que puedan desarrollarse en una variedad de fuentes astrofísicas con diferentes escalas espaciales, desde objetos estelares jóvenes (YSOs, por Young Stellar Objects) hasta cúmulos de galaxias, pasando por microcuasares (MQs, por Microquasars) y AGNs. En particular, nos hemos concentrado en estos cuatro tipos de objetos para analizar como las ondas de choque pueden acelerar partículas cargadas hasta velocidades relativistas y como luego estas partículas pueden interactuar con el medio y producir fotones mediante procesos radiativos no térmicos. Hemos calculado el espectro de fotones producido, haciendo énfasis en el análisis de la emisión de rayos gamma.

- En los YSOs masivos, estudiamos la aceleración de partículas en los choques terminales de los jets, producidos cuando éstos son frenados por el medio circundante. Hemos calculado el espectro de emisión de rayos gamma de la fuente IRAS 16547-4247 obteniendo niveles de luminosidad detectables con los telescopios que actualmente están funcionando.

- En los microcuasares de alta masa (HMMQ, por High Mass Microquasar), el viento de la estrella compañera puede tener estructura (inhomogeneidades en la densidad). Estas inhomogeneidades al llegar al jet pueden penetrar en él y producir ondas de choque. Las partículas allí aceleradas pueden producir emisión variable y, en rayos gamma detectable por los telescopios actuales. Este mecanismo podría explicar cierta variabilidad observada en fuentes como Cygnus X-1, LS 5039 y LSI +31 603.

\footnotetext{
${ }^{3}$ En frecuencias gamma, para que una detección sea confiable se requiere una relación se nal (S) ruido (N) tal que $S / N \geq 6$.
} 


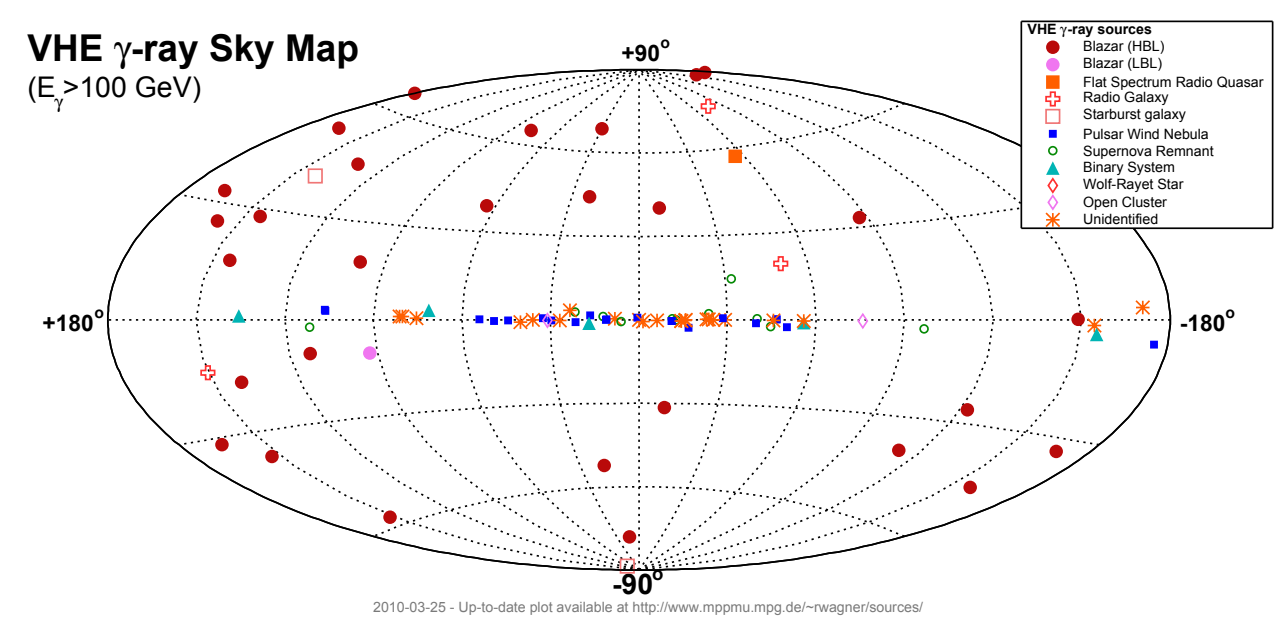

Figura 1.1: Mapa del cielo en rayos gamma de VHE. Se muestran las fuentes puntuales detectadas hasta la actualidad por diferentes telescopios. Entre las fuentes identificadas, las más comunes son los AGN, en particular, los blazares (http://www.mppmu.mpg.de/ rwagner/sources/).

- En los AGNs estudiamos una situación similar a la analizada en los HMMQs. Sin embargo, en los primeros, sería la interacción de las nubes que se encuentran orbitando al agujero negro central con el jet la que daría como resultado la emisión de rayos gamma. Este mecanismo podría explicar la emisión observada en algunas galaxias.

- Finalmente, en el cúmulo de galaxias Abell 3376 estudiamos el espectro en rayos gamma producido por la población de partículas relativistas generada en choques detectados en el borde de la fuente.

Los resultados de nuestra investigación sobre "Radiación no térmica asociada a las ondas de choque astrofísicas", temática que da nombre a esta tesis, serán presentados de la siguiente manera: en los Capítulos 2 y 3 haremos una somera descripción de los procesos no térmicos: ondas de choque y radiación. Los cuatro capítulos siguientes están dedicados a cada una de las fuentes estudiadas en esta tesis: YSOs (Capítulo 4), HMMQs (Capítulo 5), AGNs (Capítulo 6) y cúmulos de galaxias (Capítulo 7). Finalmente, en el Capítulo 8, discutiremos los resultados y las conclusiones. A lo largo de toda la tesis, los valores de las magnitudes físicas consideradas estarán dados en unidades cgs y las unidades electromagnéticas seran Gauss $(G)$ y esu. 


\section{Parte I}

\section{Procesos no térmicos}





\section{Capítulo 2}

\section{Ondas de choque y aceleración de partículas}

\subsection{Introducción}

En un plasma que se mueve a una velocidad $\vec{v}$, tiene un campo magnético $\vec{B}$, una presión $P$ y una densidad $\rho$, en el cual pueda aplicarse la aproximación magnetohidrodinámica 1 (MHD), las ecuaciones que describen el estado del sistema pueden separarse en 3 grupos: mecánicas, electromagnéticas y termodinámicas.

- Las mecánicas incluyen la conservación de la masa

$$
\frac{\mathrm{D} \rho}{\mathrm{Dt}}+\rho \vec{\nabla} \cdot \vec{v}=0
$$

y la ecuación de movimiento

$$
\rho \frac{\mathrm{D} \vec{v}}{\mathrm{D} t}=-\vec{\nabla} P+\vec{I} \times \vec{B}+\vec{F}^{\prime}
$$

donde $\vec{F}^{\prime}$ incluye a todas las demás fuerzas, además del gradiente de $P$ y de la fuerza de Lorentz $(\vec{I} \times \vec{B})$, que actúan sobre el sistema. La fuerza $\vec{F}^{\prime}$ podría ser la gravedad, la fuerza viscosa, etc. Por otro lado, la derivada convectiva $\mathrm{D} / \mathrm{D} t$ se define como $\mathrm{D} / \mathrm{D} t \equiv \partial / \partial t+\vec{v} \cdot \vec{\nabla}$. En la aproximación MHD, la relación entre $\vec{I}$ y $\vec{B}$ está dada por la ecuación de Ampere-Maxwell, $\left(4 \pi / c^{2}\right) \vec{I}=\vec{\nabla} \times \vec{B}$.

- Dentro de las ecuaciones electromagnéticas incluimos a la de divergencia nula:

$$
\vec{\nabla} \cdot \vec{B}=0
$$

\footnotetext{
${ }^{1}$ La aproximación magnetohidrodinámica considera que el campo magnético es relevante en la dinámica del plasma. En las ecuaciones de Maxwell, esto se introduce despreciando la corriente de desplazamiento.
} 
y a la ecuación de inducción

$$
\frac{\partial \vec{B}}{\partial t}=\vec{\nabla} \times(\vec{v} \times \vec{B})+\frac{c^{2}}{4 \pi \sigma_{\mathrm{B}}} \nabla^{2} \vec{B},
$$

donde $\sigma_{\mathrm{B}}$ es la conductividad magnética.

- Finalmente las ecuaciones termodinámicas son la ecuación de estado, por ejemplo la de gas ideal,

$$
P=n K_{\mathrm{B}} T,
$$

y la ecuación de conservación de la energía:

$$
\frac{\rho^{\gamma_{\mathrm{ad}}}}{\gamma_{\mathrm{ad}}-1} \frac{\mathrm{D}}{\mathrm{Dt}}\left(\frac{P}{\rho^{\gamma_{\mathrm{ad}}}}\right)=-\mathcal{L},
$$

donde $\gamma_{\text {ad }}$ es el índice adiabático y toma el valor $5 / 3$ para gases monoatómicos no relativistas y $4 / 3$ para los relativistas. Las pérdidas de energía $\mathcal{L}$ pueden estar debidas a la viscosidad, a la difusión térmica o a la radiación. En la ecuación (2.5) $n=\rho / m$ es la densidad numérica de partículas de masa $m, T$ es la temperatura y $K_{\mathrm{B}}$ la constante de Boltzmann.

Si provocamos una pequeña perturbación en el movimiento del plasma, las propiedades del sistema se verán modificadas de la siguiente manera:

$$
\xi=\xi_{0}+\xi_{1}(\vec{r}, t)
$$

donde $\xi_{1}(\vec{r}, t)$ es la perturbación respecto del estado de equilibrio $\xi_{0}$. La magnitud $\xi$ puede ser la presión, la densidad, la velocidad o el campo magnético. Si la perturbación es chica, es decir, $\left|\xi_{1}\right| \ll\left|\xi_{0}\right|$, entonces las ecuaciones de la MHD perturbadas se pueden linealizar considerando sólo los términos de primer orden en las perturbaciones $\xi_{1}$. Si no hay campos magnéticos, la perturbación satisface la ecuación de onda:

$$
\nabla^{2} \xi_{1}-\frac{1}{C^{2}} \frac{\partial^{2} \xi_{1}}{\partial t^{2}}=0
$$

donde $C$ es (el módulo de) la velocidad de propagación de la perturbacióñ2.

La ecuación de movimiento (2.2) depende de las fuerzas que actúen sobre el sistema: el gradiente de presión, la fuerza de Lorentz u otro tipos de fuerzas $\vec{F}^{\prime}$. Dependiendo de la importancia relativa de unas respecto de las otras, tendremos diferentes tipos de ondas:

- Si $|\vec{\nabla} P| \gg|\vec{I} \wedge \vec{B}|$ y $|\vec{\nabla} P| \gg\left|\vec{F}^{\prime}\right|$, y consideramos un fluido inicialmente en reposo, entonces tendremos variaciones en $P, \rho$ y $v$. Estas son las llamadas ondas de sonido y se propagan a la velocidad del sonido $C_{\mathrm{s}}=\sqrt{\gamma_{\mathrm{ad}} P / \rho}$ en el medio no perturbado.

\footnotetext{
${ }^{2} \mathrm{~A}$ los módulos de las magnitudes vectoriales los representamos a través de la letra usada para representar a la magnitud pero sin la flecha que indica la naturaleza vectorial de la misma. Por ejemplo, $|\vec{A}|=A$.
} 
- Si $|\vec{I} \wedge \vec{B}| \gg|\vec{\nabla} P|$ y $|\vec{I} \wedge \vec{B}| \gg\left|\vec{F}^{\prime}\right|$, entonces tendremos las llamadas ondas magnetohidrodinámicas o de Alfvén. Estas tienen diferentes modos dependiendo de que la variación sea en la dirección o en la intensidad de $\vec{B}$. Las ondas de Alfvén de corte son transversales (se propagan perpendicularmente a $\vec{B}_{0}$ ) y varían sólo la dirección de $\vec{B}$. La velocidad de fase de estas ondas es $\vec{v}_{\mathrm{f}}= \pm v_{\mathrm{A}} \cos \left(\theta_{\mathrm{B}}\right) \vec{k} / k$, donde $\vec{k}$ es el vector de onda y $\theta_{\mathrm{B}}$ es el ángulo entre $\vec{k}$ y $\vec{B}_{0}$, y la velocidad de grupo es $\vec{v}_{\mathrm{g}}=\vec{v}_{\mathrm{A}}$. La velocidad de Alfvén se define de la siguiente manera: $v_{\mathrm{A}}=\sqrt{\gamma_{\mathrm{ad}} B_{0} / \rho_{0}}$. Por otro lado, las ondas de Alfvén de compresión son longitudinales; modifican tanto la dirección como la intensidad de $\vec{B}$ y se propagan con una velocidad $\vec{v}_{\mathrm{f}}=\vec{v}_{\mathrm{g}}= \pm v_{\mathrm{A}} \vec{k} / k$.

- $\mathrm{Si}|\vec{I} \wedge \vec{B}| \sim|\vec{\nabla} P|$ y $\left|\vec{F}^{\prime}\right| \sim 0$, entonces tenemos las llamadas ondas magnetoacústicas. La velocidad $C_{\mathrm{sA}}$ de propagación (de fase) de estas ondas es $0 \leq C_{\mathrm{sA}} \leq \sqrt{V_{\mathrm{A}}^{2}+C_{\mathrm{s}}^{2}}$. Cuando máx $\left\{v_{\mathrm{A}}, C_{\mathrm{s}}\right\} \leq C_{\mathrm{sA}} \leq \sqrt{V_{\mathrm{A}}^{2}+C_{\mathrm{s}}^{2}}$ se dice que la onda está en el modo rápido, mientras que si $0 \leq C_{\mathrm{sA}} \leq \operatorname{mín}\left\{v_{\mathrm{A}}, C_{\mathrm{s}}\right\}$ se dice que está en el modo lento.

Notemos que en todos estos casos, la velocidad de fase, $\vec{v}_{\mathrm{f}}$, y por lo tanto la de grupo, son constantes. Esto implica que no hay dispersión, es decir, que el perfil de las ondas no cambia, contrariamente a lo que ocurre, como veremos luego, en las ondas de choque. Hasta aquí hemos realizado un breve resumen de lo que sucede cuando las perturbaciones son pequeñas. ¿Qué ocurre cuando las perturbaciones ya no son tan chicas, comenzando a hacerse importantes los términos no lineales?

\subsection{Definición y estructura de las ondas de choque}

Dada una onda sonora o una onda magnetoacústica (de compresión), cuando la amplitud es grande, es decir cuando los términos no lineales de las ecuaciones son importantes, el perfil de la onda se modifica con el tiempo. Esto se debe a que las perturbaciones de mayor amplitud viajan más rápidamente que las de menor amplitud, es decir $\vec{v}_{\mathrm{g}}$ no es constante, y el perfil de la onda se modifica empinándose cada vez más a medida que la onda avanza, como se muestra en la Figura 2.1 (Priest 1982, Platzeck 2009).

Este comportamiento es similar tanto en $\vec{v}$ como en $P$ y en $\rho$. Los gradientes de estas cantidades se hacen cada vez más grandes de tal manera que efectos disipativos como la viscosidad o el término disipativo de la ecuación de inducción no pueden despreciarse. Son estos efectos los que impiden que esta situación continúe indefinidamente. Cuando la fuerza viscosa (no consideraremos disipación magnética) se equilibra con los gradientes de $\vec{v}, P$ y $\rho$ se alcanza el estado estacionario y se frena el empinamiento del perfil de la onda. Luego continúa propagándose con este perfil constante y empinado. Esto es lo que llamamos una onda de choque. Cuando se llega a esta situación, la velocidad de propagación, que llamaremos $v_{\mathrm{ch}}$, es mayor que la velocidad de la onda linealizada correspondiente, $C_{\mathrm{s}}$ o $C_{\mathrm{sA}}$.

El equilibrio entre la fuerza viscosa y los gradientes ocurre en una región muy peque na comparada con el tamaño del sistema. El ancho $\Delta x$ de esta región de transición se estima que es del orden de algunos caminos libres medios $\lambda$ (longitud que recorre una partícula 


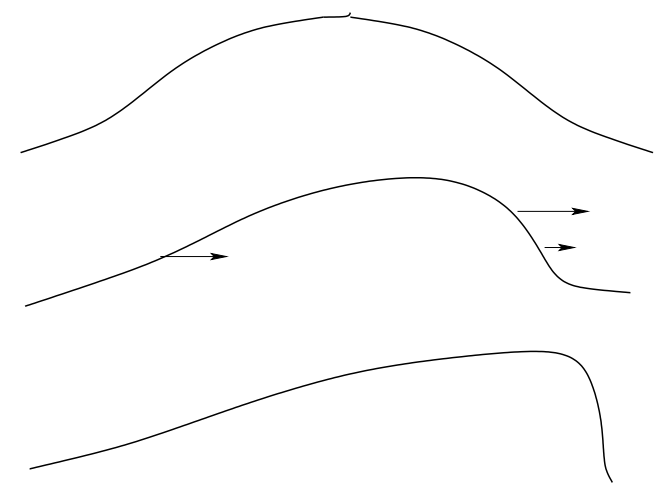

Figura 2.1: Empinamiento del frente de la onda a medida que los términos no lineales de las ecuaciones perturbadas se hacen más importantes.

antes de desviarse significativament $\left.3^{3}\right)$ y decrece a medida que $\vec{v}_{\mathrm{ch}}$ crece. De esta manera, si $v_{\mathrm{ch}} \gg C_{\mathrm{s}}$ el choque se dice fuerte y la región de transición puede considerarse como una "superficie" de discontinuidad. El tiempo que tarda el fluido en atravesar la región de transición es muy chico comparado con el tiempo de los cambios en las regiones fuera de ella. De esta manera podemos considerar que el material que se encuentra fuera de la región de transición está en un estado estacionario, como se muestra en la Figura 2.2. En la aproximación hidrodinámica (HD) y MHD $\Delta x \sim 0$. Podemos definir a las ondas de choque de la siguiente manera:

- Onda de choque: Onda de gran amplitud que produce una discontinuidad en los valores de las funciones que representan a las propiedades del plasma. El frente de choque divide al medio en dos regiones: chocada y no chocada, en las cuales los valores de $P, \rho, T, \vec{v}$ y $\vec{B}$ son muy distintos.

Denominamos con el subíndice 0 a las propiedades en el medio sin perturbar (delante del frente de onda) y con el subíndice 1 a aquellas en el medio chocado (detrás del frente de onda). Suponemos que ambos medios son uniformes. En el sistema de referencia (SR) con el medio 0 en reposo, el choque se mueve con una velocidad $\vec{U}$ y el medio perturbado con una velocidad $\vec{U}_{1}$. En el SR fijo al frente de choque, el medio no perturbado se acerca al choque con una velocidad $\vec{v}_{0}$ mientras que el material perturbado se aleja del choque con una velocidad $\vec{v}_{1}$. En la Figura 2.3 se esquematizan ambas situaciones. Notar que

$$
\vec{v}_{0}=-\vec{U} \quad \text { y } \quad \vec{v}_{1}=\vec{U}_{1}-\vec{v}_{0}
$$

Los módulos de las velocidades se relacionan de la siguiente manera: $v_{0}=U$ y $v_{1}=$ $v_{0}-U_{1}=U-U_{1}$.

\footnotetext{
${ }^{3} \mathrm{Si}$ el mecanismo de interacción dominante entre las partículas es colisional, entonces $\lambda$ es la distancia recorrida por la partícula antes de interactuar con otra. En el próximo capítulo (ver ecuación (3.6)) definiremos $\lambda$ de una manera más apropiada.
} 

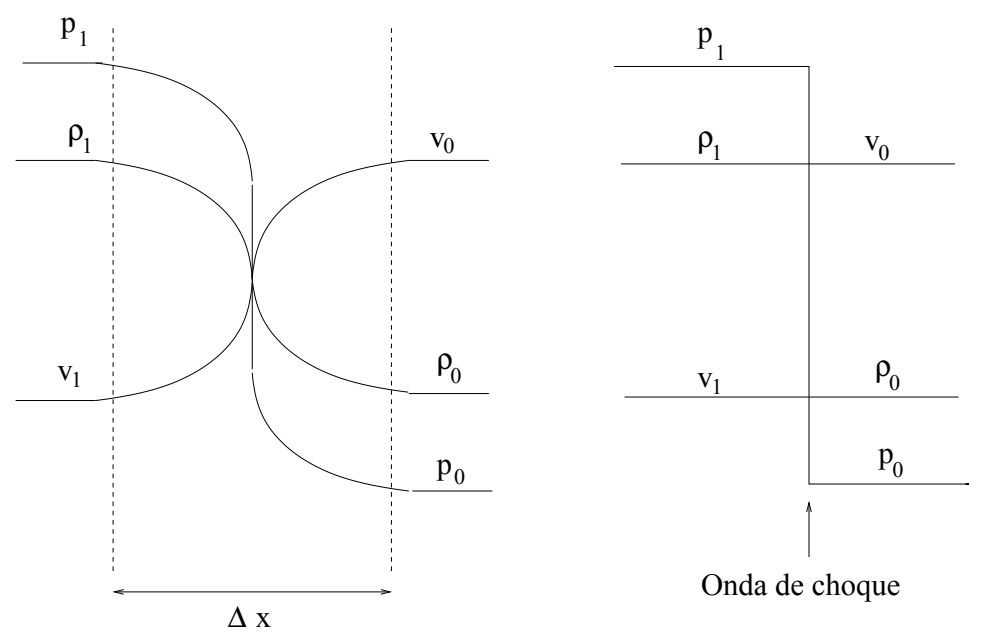

Figura 2.2: Esquema de las variaciones en la presión $(P)$, la densidad $(\rho)$, y la velocidad $(\vec{v})$ del fluido. Con los subíndices 0 y 1 indicamos las magnitudes en la región no perturbada y perturbada, respectivamente. En la figura de la izquierda se muestra la region de transición $\Delta x$ ampliada con respecto a la figura de la derecha. En HD y MHD, $\Delta x \rightarrow 0$, es decir, la región de transición puede considerarse como superficie de discontinuidad de las magnitudes graficadas.
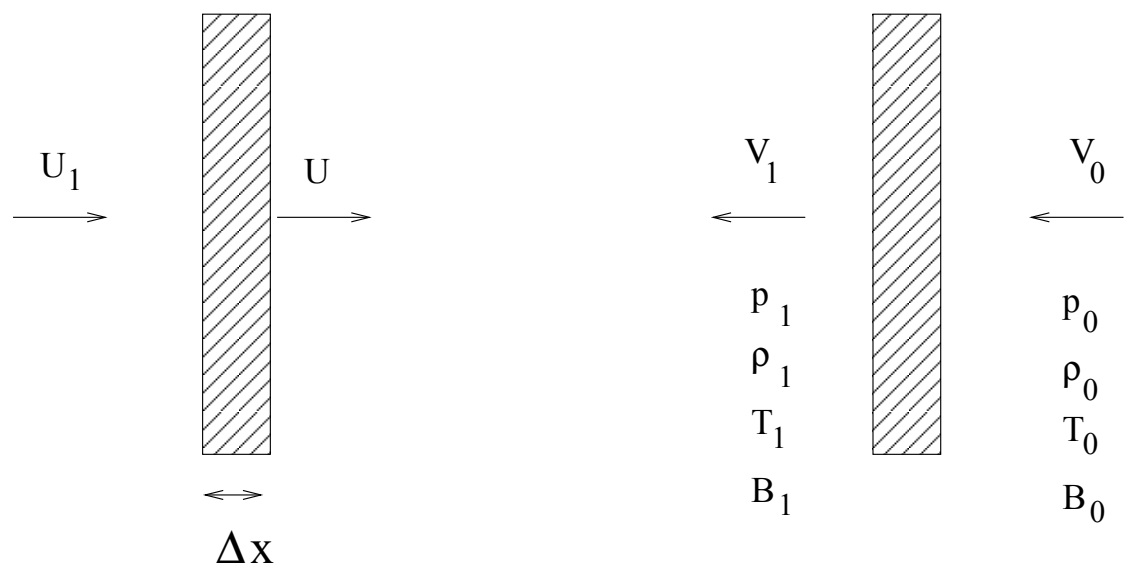

Figura 2.3: Velocidades en el SR con el medio no perturbado en reposo (izquierda) y en el SR solidario al choque (derecha). 


\subsection{Ondas de choque no relativistas}

Las ondas de choque no relativistas (NR) son aquellas tales que $v_{\text {ch }} \lesssim 0.1 c$, siendo $c$ la velocidad de la luz en el vacío. Veremos a continuación que en el caso de choques no relativistas los valores de la velocidad, la presión, la densidad y el campo magnético en los medios chocado y no chocado pueden relacionarse teniendo en cuenta las ecuaciones (2.1)-(2.6) .

\subsubsection{Ondas de choque hidrodinámicas}

Las ondas de choque hidrodinámicas son aquellas que se producen en un medio en el cual la fuerza dinámicamente relevante es $\vec{\nabla} P$. Esto es $|\vec{\nabla} P| \gg|\vec{I} \wedge \vec{B}|$ y $|\vec{\nabla} P| \gg\left|\vec{F}^{\prime}\right|$. Así, las ondas de choque hidrodinámicas se propagan a una velocidad mayor que las ondas de sonido, es decir, $v_{\mathrm{ch}}>C_{\mathrm{s}}$.

Consideremos un medio no conductor y en estado estacionario, con densidad $\rho_{0}$, presión $P_{0}$, campo magnético $\vec{B}_{0} \sim 0 \mathrm{y}$ un choque propagandose con una velocidad $\vec{v}_{\text {ch }}$. En el SR solidario al frente de choque, la velocidad del medio no chocado es $\vec{v}_{0}$ y debido a que hemos tomado convenientemente el eje $x$ como la dirección de propagación del choque, sólo habrá variaciones espaciales en tal dirección. En este caso de ondas de choque propagándose en un medio con $\vec{B} \sim 0$, las ecuaciones que describen el estado del sistema son la ecuación (2.1) de conservación de la masa, la ecuación (2.2) de movimiento y la de conservación de la energía (2.6).

Debido a que el sistema está en estado estacionario, la ecuación (2.1) se simplifica a

$$
\frac{\mathrm{d}(\rho \vec{v})}{\mathrm{d} x}=0 .
$$

Esta condición debe cumplirse a lo largo de cualquier línea de fluído, por lo cual, si la integramos entre un punto en la región $1\left(x_{1}\right)$ y otro en la región no perturbada $\left(x_{0}\right)$ obtenemos:

$$
\rho_{1} \vec{v}_{1}=\rho_{0} \vec{v}_{0} .
$$

Esta ecuación describe que el flujo de masa $\rho \vec{v}$ que pasa a través de la onda de choque se conserva. Por otro lado, la ecuación de movimiento en un medio no conductor $(\vec{I} \wedge \vec{B}=0)$ y estacionario se reduce a

$$
\rho \vec{v} \cdot \frac{\mathrm{d} \vec{v}}{\mathrm{~d} x}=-\frac{\mathrm{d} p}{\mathrm{~d} x}+\frac{4}{3} \frac{\mathrm{d}}{\mathrm{d} x}\left(\lambda_{\mathrm{v}} \cdot \frac{\mathrm{d} \vec{v}}{\mathrm{~d} x}\right)
$$

La fuerza viscosa $\vec{F}^{\prime}=-(4 / 3) \mathrm{d}\left(\lambda_{v} \mathrm{~d} v / \mathrm{d} x\right) / \mathrm{d} x \grave{i}$, donde $\lambda_{v}$ es una constante, está presente en la región de transición $\Delta x$ pero es nula tanto en el medio chocado como en el no chocado. Por lo tanto, si integramos la ecuación (2.12) entre $x_{1}$ y $x_{0}$ y usamos que $\rho_{1} v_{1}=\rho_{0} v_{0}$, obtenemos

$$
\rho_{0} v_{0}^{2}+P_{0}=\rho_{1} v_{1}^{2}+P_{1} .
$$


La magnitud $(\rho \vec{v}) \cdot \vec{v}$, conocida como "presión cinética", es la cantidad de impulso transportado por unidad de tiempo y por unidad de área transversal al movimiento. Por otro lado, $P$ es la fuerza que actúa sobre dicha área.

Finalmente, en la ecuación de conservación de la energía consideraremos las pérdidas por conducción térmica $\mathcal{L}_{\mathrm{c}}=\vec{\nabla}\left(-\lambda_{\mathrm{c}} \vec{\nabla} T\right)$, donde $\lambda_{\mathrm{c}}$ es una constante, y por viscosidad $\mathcal{L}_{\mathrm{v}}=(-2 / 3) \rho \lambda_{\mathrm{v}}(\vec{\nabla} \cdot \vec{v})^{2}$, ambas presentes solo en la región de transición. La ecuación (2.6) escrita en función de la energía interna por unidad de masa $\epsilon$ y teniendo en cuenta la viscosidad y la conducción de calor toma la forma

$$
\rho v\left(\frac{\mathrm{d} \epsilon}{\mathrm{d} x}-\frac{p}{\rho^{2}} \frac{\mathrm{d} \rho}{\mathrm{d} x}\right)=\frac{\mathrm{d}}{\mathrm{d} x}\left(\lambda_{\mathrm{c}} \frac{\mathrm{d} T}{\mathrm{~d} x}\right)+\frac{2}{3} \rho \lambda_{\mathrm{v}}\left(\frac{\mathrm{d} v}{\mathrm{~d} x}\right)^{2} .
$$

Integrando como antes entre $x_{1}$ y $x_{0}$, donde $\mathrm{d} T / \mathrm{d} x=0 \mathrm{y} \mathrm{d} \vec{v} / \mathrm{d} x=0$, y usando las relaciones (2.11) y (2.13), obtenemos:

$$
\left(\frac{1}{2} \rho_{1} v_{1}^{2}+\epsilon_{1} \rho_{1}\right) \vec{v}_{1}+P_{1} \vec{v}_{1}=\left(\frac{1}{2} \rho_{0} v_{0}^{2}+\epsilon_{0} \rho_{0}\right) \vec{v}_{0}+P_{0} \vec{v}_{0},
$$

donde $\left((1 / 2) \rho v^{2}+\epsilon \rho\right)$ es la cantidad densidad de energía cinética e interna transportada por unidad de superficie y de tiempo; $p v$ es el trabajo por unidad de tiempo y de área realizado por la presión del gas. Si consideramos que $\rho_{1} \vec{v}_{1}=\rho_{0} \vec{v}_{0}$ tenemos

$$
\epsilon_{1}+\frac{P_{1}}{\rho_{1}}+\frac{v_{1}^{2}}{2}=\epsilon_{0}+\frac{P_{0}}{\rho_{0}}+\frac{v_{0}^{2}}{2} .
$$

Teniendo en cuenta que para un gas ideal $\epsilon=p / \rho(\gamma-1)$, la ecuación de conservación de la energía nos da la relación

$$
\frac{\gamma_{\text {ad }}}{\gamma_{\text {ad }}-1} \frac{P_{1}}{\rho_{1}}+\frac{v_{1}^{2}}{2}=\frac{\gamma_{\text {ad }}}{\gamma_{\text {ad }}-1} \frac{P_{0}}{\rho_{0}}+\frac{v_{0}^{2}}{2} .
$$

Así hemos obtenido las relaciones de Rankine-Hugoniot, o relaciones de salto:

$$
\begin{aligned}
\rho_{0} \vec{v}_{0} & =\rho_{1} \vec{v}_{1} \\
\rho_{0} v_{0}^{2}+P_{0} & =\rho_{1} v_{1}^{2}+P_{1} \\
\frac{\gamma_{\text {ad }} P_{0}}{\left(\gamma_{\text {ad }}-1\right) \rho_{0}}+\frac{1}{2} v_{0}^{2} & =\frac{\gamma_{\text {ad }} P_{1}}{\left(\gamma_{\text {ad }}-1\right) \rho_{1}}+\frac{1}{2} v_{1}^{2} .
\end{aligned}
$$

Resolviendo estas ecuaciones podemos obtener información sobre como la onda de choque modifica el medio. De la ecuación (2.18) obtenemos que

$$
\frac{v_{1}}{v_{0}}=\frac{\rho_{0}}{\rho_{1}} .
$$

Por otro lado, de las ecuaciones (2.19) y (2.20) podemos hallar expresiones para $\rho_{0} / \rho_{1}$ y para $P_{0} / P_{1}$ en función del número de Mach $M_{0}$ en el medio no perturbado:

$$
M_{0}=\frac{v_{0}}{C_{\mathrm{s}_{0}}}=\frac{v_{0}}{\sqrt{\gamma_{\mathrm{ad}} P_{0} / \rho_{0}}} .
$$


Se obtienen las siquientes relaciones (Landau \& Lifshitz, 1959):

$$
\begin{gathered}
\frac{\rho_{1}}{\rho_{0}}=\frac{v_{0}}{v_{1}}=\frac{\left(\gamma_{\mathrm{ad}}+1\right) M_{0}^{2}}{\left(\gamma_{\mathrm{ad}}-1\right) M_{0}^{2}+2} \\
\frac{P_{1}}{P_{0}}=\frac{2 \gamma_{\mathrm{ad}} M_{0}^{2}-\left(\gamma_{\mathrm{ad}}-1\right)}{\gamma_{\mathrm{ad}}+1} .
\end{gathered}
$$

Si además consideramos la ecuación de estado (2.5), entonces la temperatura del medio chocado es $T_{1}=P_{1} /\left(K_{\mathrm{B}} n_{1}\right)$ y en función de parámetros conocidos el cociente $T_{1} / T_{0}$ resulta:

$$
\frac{T_{1}}{T_{0}}=\frac{\left[2 \gamma_{\mathrm{ad}} M_{0}^{2}-\left(\gamma_{\mathrm{ad}}-1\right)\right]\left[\left(\gamma_{\mathrm{ad}}-1\right) M_{0}^{2}+2\right]}{\left(\gamma_{\mathrm{ad}}+1\right)^{2} M_{0}^{2}} .
$$

Recordemos que todas estas expresiones (2.23)-(2.25) han sido deducidas en el SR que se mueve con el frente de choque. Teniendo en cuenta que $v_{1} \leq v_{0}$, podemos decir:

- La onda de choque comprime el gas: $\rho_{1}>\rho_{0}$.

- La velocidad de la onda de choque es supersónica: $U=v_{0}>C_{\mathrm{s}_{0}}$.

- El material chocado es subsónico: $M_{1}<1$.

- La onda de choque aumenta la presión del medio: $P_{1}>P_{0}$.

- La entropía aumenta: $S_{1}>S_{0}$.

- La onda de choque calienta el plasma: $T_{1}>T_{0}$.

Si bien la presión crece si la velocidad del choque aumenta, el incremento de la densidad está acotado:

$$
\lim _{M_{0} \rightarrow \infty} \frac{\rho_{1}}{\rho_{0}}=\frac{\gamma_{\mathrm{ad}}+1}{\gamma_{\mathrm{ad}}-1} .
$$

Para el caso particular de un gas monoatómico, $\gamma_{\text {ad }}=5 / 3$ y se obtiene $\rho_{1} / \rho_{0}=4$. Cuando $M_{0} \gg 1$ se dice que el choque es fuerte y en este caso las relaciones de salto se expresan de una manera muy sencilla:

$$
\begin{gathered}
\rho_{1}=4 \rho_{0} \quad \text { y } \quad v_{1}=\frac{v_{0}}{4}, \\
P_{1}=\frac{3}{4} \rho_{0} v_{0}^{2} \quad \text { y } \quad T_{1} \sim 2 \times 10^{-9} v_{0}^{2} \mathrm{~K},
\end{gathered}
$$

y estas relaciones valen en toda la región chocada.

Los choques que hemos estudiado en esta sección se conocen como choques adiabáticos, ya que hemos considerado que no hay pérdidas de energía ni en la región chocada ni en la no chocada, es decir, $\mathcal{L}_{1}=\mathcal{L}_{0}=0$. Sin embargo, hay situaciones en las cuales las condiciones en la región 1 (originalemente adiabática) son tales que las pérdidas radiativas comienzan a hacerse relevantes. En este caso, la propagación del choque se modifica disminuyendo $v e c v_{\mathrm{ch}} \mathrm{y}$ el estado del medio 1 cambia, haciéndose mucho más denso y frío, como veremos a continuación. 


\section{Choques radiativos}

Dado un choque que se propaga a una velocidad $\vec{v}_{\text {ch }}$, y cuyas perturbaciones en el medio están descriptas a través de las ecuaciones (2.23), (2.24) y (2.25), estas últimas pueden no valer en toda la región chocada si las pérdidas radiativas en dicho medio son importantes. Si bien en la región más cercana al choque el estado del medio es aquel descripto por las condiciones de un choque adiabático, a mayores distancias la densidad crece y la temperatura disminuye. La región 1 es entonces muy densa y fría.

Para estimar la importancia de las pérdidas radiativas en el medio chocado debemos comparar el tiempo de enfriamiento $t_{\text {ter }}$ con otros tiempos característicos de cada sistema. Si consideramos las pérdidas por emisión térmica (de líneas y continuo) $\mathcal{L}_{\text {ter }} \sim n^{2} \Lambda(T)$, donde $\Lambda(T)=A_{\mathrm{t}} T^{\alpha_{\mathrm{t}}}$ (Myasnikov et al. 1998):

$$
\Lambda(T)= \begin{cases}7 \times 10^{-19} T^{-0.6} & \text { si } 10^{4}<T<4 \times 10^{7} \mathrm{~K} \\ 3 \times 10^{-27} T^{0.5} & \text { si } T>4 \times 10^{7} \mathrm{~K},\end{cases}
$$

entonces $t_{\text {ter }}=(5 / 2) P_{1} / \mathcal{L}_{\text {ter }}$. Por otro lado, si consideramos como tiempo característico aquel en el cual el material chocado recorre una distacia característica $X$, entonces $t_{\mathrm{d}} \sim$ $X / U_{1}$ y el choque será radiativo si $t_{\text {ter }}<t_{\mathrm{d}}$.

En este caso, debido a las pérdidas radiativas, la energía no se conserva a lo largo de una línea de fluído en la región chocada. La ecuación (2.6) se escribe ahora como (Zhekov \& Palla, 2007)

$$
\frac{\mathrm{d}}{\mathrm{d} x}\left(\frac{\gamma_{\mathrm{ad}}}{\gamma_{\mathrm{ad}}-1} P_{1} v_{1}+\frac{\rho_{1} v_{1}^{3}}{2}\right)=-\mathcal{L}_{\text {ter }},
$$

y si consideramos que $P_{1}$ se mantiene constante, entonces la ecuación anterior puede reescribirse como

$$
\frac{5}{2} n_{1} v_{1} K_{\mathrm{B}} \frac{\mathrm{d} T}{\mathrm{~d} x} \sim-\mathcal{L}_{\text {ter }}=-n_{1}^{2} \Lambda\left(T_{1}\right) .
$$

Resolviendo esta ecuación obtenemos el siguiente perfil de temperatura en la región chocada:

$$
T_{1}(x)=\left(-\frac{2 P_{1}^{2}}{5 n_{0} v_{0} K_{\mathrm{B}}^{3}} A_{\mathrm{t}}\left(3-\alpha_{\mathrm{t}}\right) x+\left(T_{1}^{\mathrm{ad}}\right)^{3-\alpha_{\mathrm{t}}}\right)^{1 /\left(3-\alpha_{\mathrm{t}}\right)},
$$

donde $P_{1}$ y $T_{1}^{\text {ad }}$ son la presión y la temperatura obtenidas con las relaciones de RankineHugoniot. En la Figura 2.4 se muestra el perfil de temperatura en la región chocada de un choque radiativo obtenido bajo la aproximación de presión constante en dicha región. Conociendo $T_{1}(x)$, la densidad puede hallarse a partir de la ecuación de estado. Para fluidos ideales la densidad en la región 1 varía de la siguiente manera:

$$
n_{1}(x)=\frac{P_{1}}{K_{\mathrm{B}} T_{1}(x)} .
$$

De las ecuaciones (2.32) y (2.33) vemos que a medida que el material se aleja del frente de choque, $T_{1}$ disminuye drásticamente (ver la Figura 2.4) y $n_{1}$ crece. De esta manera, el medio chocado se enfría en un tiempo menor que el tiempo en el cual el choque recorre una distancia del orden del tamaño del sistema. La región adiabática del medio 1 es pequeña y luego el material se enfría hasta que se llega a un estado de alta densidad; muy compacto. 


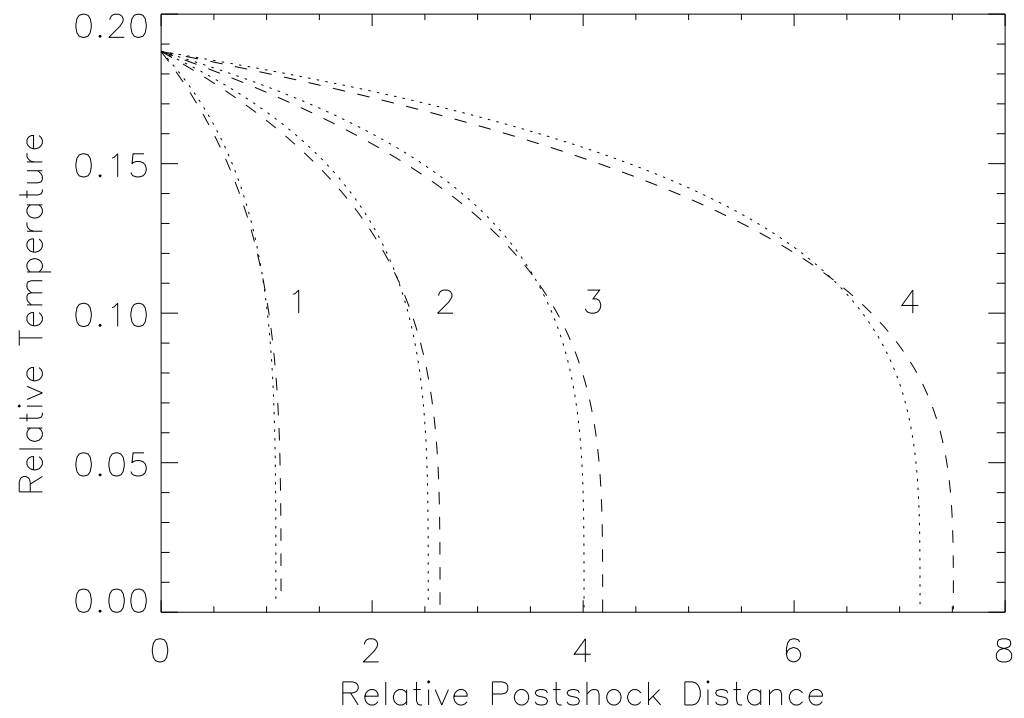

Figura 2.4: Perfil de temperatura en el medio chocado. Se muestran las soluciones exactas (línea punteada) y las calculadas con la aproximación de presión constante (línea de rayas). Los números 1, 2, 3 y 4 indican que las velocidades de los choques consideradas han sido $v_{\mathrm{ch}}=500,600,750$ y $900 \mathrm{~km} \mathrm{~s}^{-1}$ (Zhekov \& Palla, 2007). 


\subsubsection{Ondas de choque magnetohidrodinámicas}

En el caso en que $\vec{B} \neq 0$ y relevante dinámicamente, entonces tendremos ondas de choque MHD. Las hay de diferentes tipos dependiendo de la geometría de $\vec{B}$.

\section{Choques transversales}

Los choques transversales son aquellos en los cuales el campo magnético en la región no perturbada, $\vec{B}_{0}$, es perpendicular a la velocidad de propagación del choque. Es decir, $\vec{B}_{0} \perp \vec{v}_{\mathrm{ch}}$. En este caso, además de considerar las ecuaciones de movimiento, de conservación de la masa y de conservación de la energía, se considera también la ecuación de inducción. Así, aparece en las relaciones de Rankine-Hugoniot (2.19) y (2.20) el término $B^{2} / 8 \pi$ correspondiente a la presión y a la densidad de energía magnética. Se tiene la relación adicional

$$
\frac{B_{1}}{B_{0}}=\frac{\rho_{1}}{\rho_{0}} .
$$

Definiendo el parámetro $\zeta \equiv \rho_{1} / \rho_{0}$, se tiene que $\zeta$ satisface una ecuación cúbica. La solución $\zeta=1$ corresponde a una onda de Alfvén de corte si $v_{0}^{2}=v_{\mathrm{A}_{1}}^{2}$, que no son ondas de choque. (Las ondas de Alfvén de corte son ondas MHD que no se deforman aunque la amplitud de la pertubación sea grande.) La otra solución es $\zeta<0$, pero se descarta por no tener sentido físico. Finalmente, la solución $\zeta>0$ corresponde a una onda de choque. En esta situación se tiene un choque propagándose perpendicularmente a $\vec{B}$ y el efecto de este último es reducir el valor de $\zeta$ respecto del caso hidrodinámico. Es decir, en presencia de un campo magnético, el aumento de la densidad de la región chocada es menor que en el caso hidrodinámico.

\section{Choques oblicuos}

Este caso corresponde a aquel en el cual $\vec{B}_{0}$ es oblicuo a $\vec{v}_{\mathrm{ch}}$. Aquí, para obtener las relaciones de salto, además de tener en cuenta las ecuaciones consideradas en el caso de los choques transversales, usamos también la ecuación $\vec{\nabla} \cdot \vec{B}=0$.

Resolviendo como antes estas ecuaciones se llega nuevamente a una relación cúbica para $\zeta$. Una de las soluciones posibles es la trivial, $\zeta=1$, en la cual no hay onda de choque. La solución $\zeta=1$ corresponde a una onda de Alfvén si $v_{0}^{2}=v_{\mathrm{A}_{1}}^{2}$ que, como mencionamos antes, no son ondas de choque. Se las llama ondas intemedias porque se propagan a la velocidad de Alfvén en la dirección $x$, es decir, $v_{\mathrm{ch}}=v_{\mathrm{A}_{0 x}}$. Las otras dos soluciones, dadas por $\zeta>1$, corresponden a las ondas de choque asociadas a las ondas magnetoacústicas rápida $\left(v_{0 x}>v_{\mathrm{A}_{0 x}}\right)$ y lenta $\left(v_{0 x}<v_{\mathrm{A}_{0 x}}\right)$. 

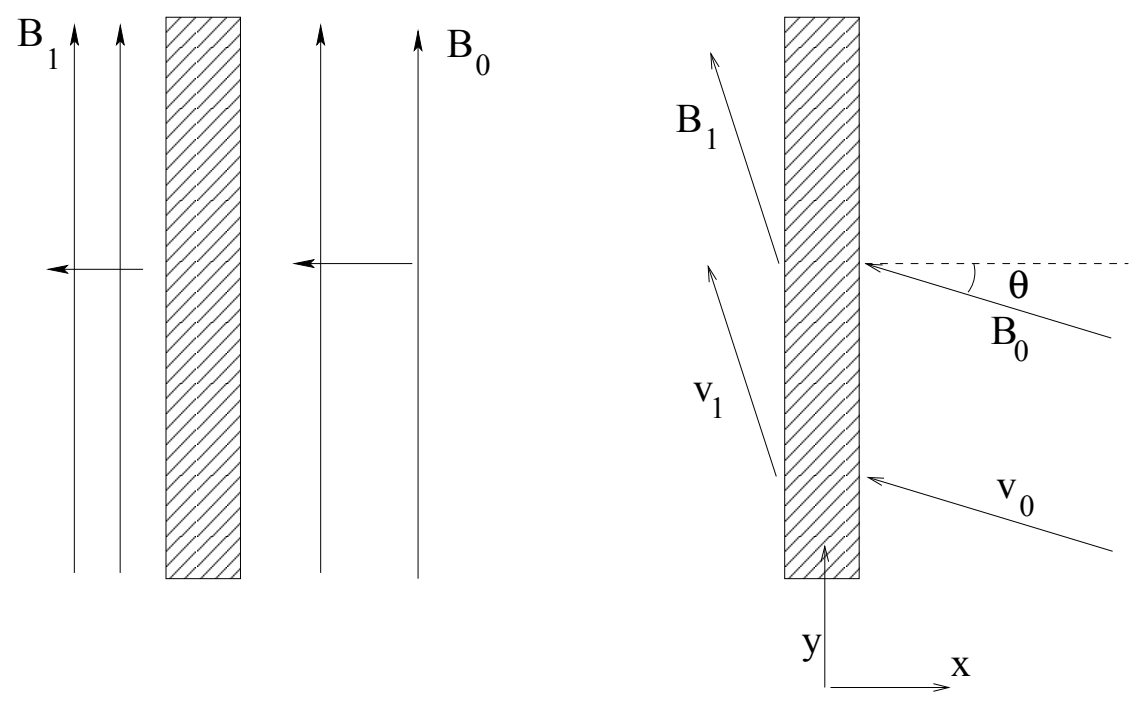

Figura 2.5: Ondas de choque transversales (izquierda) y oblicuas (derecha). El ancho del choque es $\Delta x \rightarrow 0$ y $\vec{B}_{0}$ y $\vec{B}_{1}$ son el campo magnético en la región no perturbada y perturbada, respectivamente.

\subsection{Ondas de choque relativistas}

Un fluido que se mueve con una velocidad $U \gtrsim 0.1 c$ es relativista. La información sobre su estado esta contenida en el tensor de energía-impulso $T_{\mu \nu}$. Para un fluido ideal:

$$
T_{\mu \nu}=\frac{1}{c^{2}} \omega u_{\mu} u_{\nu}+P g_{\mu \nu},
$$

donde $u_{\mu}=\Gamma(c, \vec{u})$ es la tetra-velocidad, $\beta=u / c$ y $\Gamma=1 / \sqrt{1-\beta^{2}}$ es el factor de Lorentz y $\omega=e_{\mathrm{t}}+P$ es la entalpía. La densidad de energía total $e_{\mathrm{t}}$ de un fluido relativista se escribe $e_{\mathrm{t}}=n m c^{2}+e$, donde $n m c^{2}$ es la densidad de energía en reposo y e es la cinética. Finalmente, $g_{\mu \nu}$ es la métrica del espacio-tiempo. En esta tesis consideraremos la métrica de Minkowski, con $g_{t t}=-1$ y $g_{x x}=g_{y y}=g_{z z}=1$. En el SR propio, es decir, que se mueve con el fluido, $T_{\mu \nu}$ es una matríz diagonal:

$$
T_{\mu \nu}=\left(\begin{array}{cccc}
e_{\mathrm{t}} & 0 & 0 & 0 \\
0 & P & 0 & 0 \\
0 & 0 & P & 0 \\
0 & 0 & 0 & P
\end{array}\right) .
$$

El índice adiabático para gases monoatómicos varía de la siguiente manera: $4 / 3<\gamma_{\text {ad }}<$ $5 / 3$. En el caso no relativista (NR) $\gamma_{\text {ad }}=5 / 3$ mientras que en el caso ultra-relativista (UR) $\gamma_{\text {ad }}=4 / 3$. En esta última situación, la ecuación de estado es $P=e / 3$ y la velocidad del sonido resulta $C_{\mathrm{s}}=c / \sqrt{3}$. Las ecuaciones de movimiento y de conservación de la energía 
están contenidas en la derivada $\partial T_{\mu}^{\nu} / \partial x^{\mu}=0$ mientras que la ecuación de continuidad de la masa, o de conservación de la densidad de partículas $n$, se obtiene a partir de $\partial\left(n u^{\nu}\right) / \partial x^{\nu}=$ 0 .

La teoría de las ondas de choque en fluidos relativistas se construye de la misma manera que para las NR presentadas en la Sección 2.3 (Taub 1948). Si consideramos un choque que se propaga en la dirección $x$, las condiciones de salto se obtienen de considerar la conservación del número de partículas $(n \Gamma \beta)$, y las densidades de flujo de momento $\left(\omega \Gamma^{2} \beta^{2}+P\right)$ y de energía $\left(\omega \Gamma^{2} \beta\right)$. En el SR del choque, estas ecuaciones se escriben de la siguiente manera:

$$
\begin{aligned}
n_{0} \Gamma_{0} \beta_{0} & =n_{1} \Gamma_{1} \beta_{1}, \\
\omega_{0} \Gamma_{0}^{2} \beta_{0}^{2}+P_{0} & =\omega_{1} \Gamma_{1}^{2} \beta_{1}^{2}+P_{1}, \\
\omega_{0} \Gamma_{0}^{2} \beta_{0} & =\omega_{1} \Gamma_{1}^{2} \beta_{1} .
\end{aligned}
$$

Para resolverlas consideraremos el caso UR, en el cual la ecuación de estado es $P=e / 3$ y la entalpía se reduce a $\omega=4 P$. Además consideraremos que $P_{0} \ll \omega_{0} \Gamma_{0}^{2} \beta_{0}^{2}$, es decir, un choque fuerte. En el SR del choque, el medio no chocado llega al frente de choque con una velocidad $v_{0} \sim c$, con lo cual $\beta_{0} \sim 1 \mathrm{y} \Gamma_{0} \rightarrow \infty$. El medio chocado se aleja del choque a una velocidad menor que la del medio no chocado, pero que aún es moderadamente relativista:

$$
\beta_{1}=\frac{1}{3} \quad \longrightarrow \quad \Gamma_{1}=\frac{3}{2 \sqrt{2}} \sim 1.06 .
$$

Por otro lado, la densidad chocada resulta $n_{1}=2 \sqrt{2} n_{0} \Gamma_{0}$. Pero recordemos que $n_{0}$ está medida en el SR del fluído. Si transformamos esta magnitud al SR del observador a través de la relación $n^{\prime}=\Gamma n$, donde las magnitudes primadas son las medidas en este SR, obtenemos

$$
n_{1}^{\prime} \sim 3 n_{0}^{\prime} .
$$

Finalmente, la presión en el medio chocado resulta

$$
P_{1}=\frac{2}{3} m n_{0}^{\prime} \Gamma_{0} c^{2}
$$

Al ser la presión un invariante relativista, $P_{1}=P_{1}^{\prime}$.

Si consideramos campos magnéticos, entonces, al igual que en el caso no relativista, sólo debemos hacer los siguientes reemplazos en las ecuaciones (2.38) y (2.39): $P \rightarrow P+B^{2} /(8 \pi)$ $\mathrm{y} e \rightarrow e+B^{2} /(8 \pi)$.

\subsection{Producción de ondas de choque}

Hay muchas maneras de producir ondas de choque en sistemas astrofísicos. En esta tesis, hemos estudiado aquellas que se forman por perturbar un fluido que se mueve con una velocidad mayor que la del sonido. Esto es, dado un choque que se propaga por un medio, estudiamos de que manera la interacción con el medio externo puede producir otros choques. 


\subsubsection{Ondas de choque por perturbaciones locales}

Una de las formas más comunes de perturbar un fluido en movimiento es interponiendolé un obstáculo. Si el fluído es supersónico y el obstáculo es rígido, entonces se produce una única onda de choque que se propaga en el sentido opuesto al movimento del fluido. Si el obstáculo no es rígido, se puede producir además un choque que se propaga a través del obstáculo, como se muestra en la Figura 2.6. Un escenario posible para esta interacción es un jet al cual se le interpone una inhomogeneidad del medio circundante. En esta tesis hemos estudiado dos situaciones similares: en un HMMQ y en un AGN. En el primer caso, estudiamos la interacción de grumos presentes en el viento de la estrella compañera con el jet del objeto compacto y en el segundo hemos estudiado la interacción de nubes que circundan el agujero negro del centro de la galaxia con el jet del AGN.

A fin de saber cuales son los procesos físicos más relevantes en este tipo de interacciones, haremos un análisis de las escalas de tiempo de los procesos que tienen lugar debido a la interacción de un obstáculo con un jet.

\section{Escalas de tiempo}

Supongamos un obstáculo esférico, de radio $R_{\mathrm{o}}$, con una densidad homogénea $n_{\mathrm{o}}$ y que se mueve con una velocidad $\vec{v}_{\mathrm{o}}$ en la dirección perpendicular a la dirección de propagación del jet, que designaremos $z$. Por otro lado supondremos un jet que se propaga con una velocidad $v_{\mathrm{j}}$ y con un factor de Lorentz $\Gamma_{\mathrm{j}}$. Considerando un ángulo de abertura del jet $\phi \sim 6^{\circ}$ (valor típico de jets colimados de MQs y AGNs), la relación entre el radio y la altura es $R_{\mathrm{j}} \sim 0.1 z_{\mathrm{j}}$. Considerando que la luminosidad cinética del $j e t, L_{\mathrm{j}}$, es constante, la densidad, $n_{\mathrm{j}}$ (en el SR del laboratorio), de éste varía con $z_{\mathrm{j}}$ a través de la relación

$$
L_{\mathrm{j}}=\left(\Gamma_{\mathrm{j}}-1\right) m_{p} c^{2} \sigma_{\mathrm{j}} n_{\mathrm{j}} v_{\mathrm{j}},
$$

donde $\sigma_{\mathrm{j}}=\pi r_{\mathrm{j}}^{2}$ es la sección del jet a la altura $z_{\mathrm{j}}=10 r_{\mathrm{j}}$. Un parámetro importante en nuestro estudio será el cociente entre la densidad del obstáculo y la del jet: $\chi \equiv n_{\mathrm{o}} / n_{\mathrm{j}}$.

El tiempo de penetración, $t_{\mathrm{o}}$, del obstáculo en el jet puede estimarse a través de la ecuación:

$$
t_{\mathrm{o}} \sim \frac{2 R_{\mathrm{o}}}{v_{\mathrm{o}}} .
$$

Por otro lado, si suponemos que dentro del jet el obstáculo se mueve siguiendo su trayectoria original, el tiempo que tardaría en cruzar el jet (recorriendo su ancho igual a $2 R_{\mathrm{j}}$ ) está dado por

$$
t_{\mathrm{j}} \sim \frac{2 R_{\mathrm{j}}}{v_{\mathrm{o}}} \sim \frac{2\left(0.1 z_{\mathrm{j}}\right)}{v_{\mathrm{o}}} .
$$

Una vez que el obstáculo ha entrado en el jet, la interacción con el material de este último producirá dos choques: uno en el jet y otro (o una onda sónica) en el obstáculo, como se muestra en la Figura 2.6. El choque en el primero se mueve a una velocidad $\sim v_{\mathrm{j}}$ y lo llamaremos bow shock ("choque de proa"). Al cabo de recorrer una distancia $Z$ en el jet, este choque alcanza el estado estacionario, es decir, la separación entre el bow shock y 

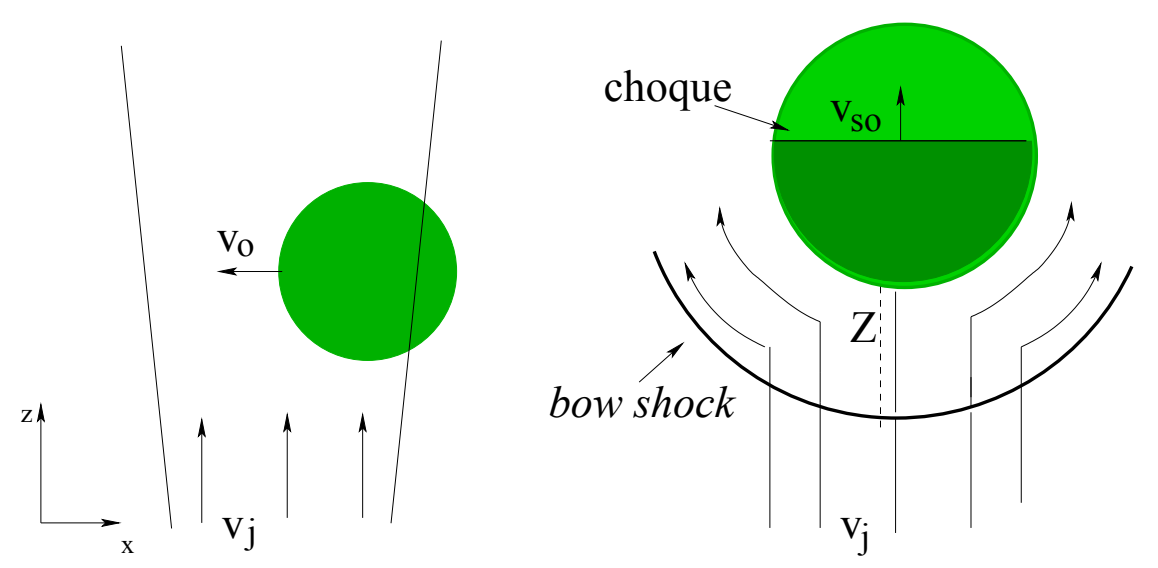

Figura 2.6: Esquema de la interacción de un jet con un obstáculo (verde). Izquierda: el obstáculo penetra en el jet moviendosé a una velocidad $\vec{v}_{\mathrm{o}}$. Derecha: Una vez que el obstáculo está completamente adentro del jet, se forma un bow shock simétrico en el jet. Mientras, un choque se propaga en el obstáculo a una velocidad $\vec{v}_{\mathrm{co}}$. El bow shock está a una distancia $Z$ del obstáculo. (El gráfico no está a escala.)

el obstáculo es fija e igual a $Z$. Para estimar esta distancia consideramos que el número de partículas del jet que por unidad de tiempo atraviesan el bow shock $\left(\sim n_{\mathrm{j}} v_{\mathrm{j}} \sigma_{\mathrm{o}}\right.$, donde $\sigma_{\mathrm{o}}=\pi R_{\mathrm{o}}^{2}$ es la sección del obstáculo) y luego escapan siendo advectadas de la región chocada a la velocidad $v_{\mathrm{j} 1}$ del medio chocado del jet $\left(v_{\mathrm{j} 1} \sim v_{\mathrm{j}} / 4\right.$ ó $v_{\mathrm{j}} / 3$, para los casos NR y UR, respectivamente) se conserva. De esta manera, de la igualdad $n_{\mathrm{j}} v_{\mathrm{j}} \sigma_{\mathrm{o}}=n_{\mathrm{j} 1} v_{\mathrm{j} 1} 2 \pi R_{\mathrm{o}} Z$, obtenemos que $Z \sim 0.2 R_{\mathrm{o}} \mathrm{y} \sim 0.3 R_{\mathrm{o}}$ en los casos de choques UR y NR, respectivamente. Luego, el tiempo en el cual el bow shock alcanza el estado estacionario es

$$
t_{\mathrm{bs}} \sim \frac{Z}{v_{\mathrm{j} 1}} .
$$

Respecto del choque en el obstáculo, la velocidad de propagación puede determinarse asumiendo que se establece el equilibrio de presiones en la superficie de separación entre la región chocada del jet y del obstáculo y usando las relaciones de salto. Se obtiene la siguiente expresión para la velocidad:

$$
v_{\mathrm{co}} \sim \frac{v_{\mathrm{j}}\left(\Gamma_{\mathrm{j}}-1\right)}{\sqrt{\chi}} .
$$

Luego el tiempo en el cual el choque recorre todo el obstáculo es

$$
t_{\mathrm{co}} \sim \frac{2 R_{\mathrm{o}}}{v_{\mathrm{co}}} \sim \frac{2 R_{\mathrm{o}} \sqrt{\chi}}{v_{\mathrm{j}}\left(\Gamma_{\mathrm{j}}-1\right)} .
$$

Esta magnitud $t_{\text {co }}$ puede considerarse un tiempo característico (o de vida) del sistema, ya que como veremos a continuación, las inestabilidades pueden destruir el obstáculo en un 
tiempo $\sim t_{\text {co }}$ o éste podría ser acelerado y comenzar a moverse con el jet antes de escapar del mismo (en un tiempo $t_{\mathrm{j}}$ ).

La aceleración $\vec{g}$ que el material del jet ejerce contra el obstáculo puede determinarse considerando conservación de la entalpía y suponiendo que toda la presión cinética del jet se convierte en presión térmica del material chocado en la superficie de contacto con el obstáculo. Así obtenemos que

$$
g \sim \frac{\left(\Gamma_{\mathrm{j}}-1\right) v_{\mathrm{j}}^{2}}{\chi R_{\mathrm{o}}},
$$

con lo cual el tiempo de aceleración del obstáculo hasta la velocidad del jet es

$$
t_{\mathrm{g}} \sim \frac{v_{\mathrm{j}}}{g} \sim \sqrt{\chi} t_{\mathrm{co}}
$$

Sin embargo, en un tiempo $\sim t_{\text {co }}$ el obstáculo se acelera hasta la velocidad $v_{\text {co }}$ del choque que se propaga en su interior. La aceleración $\vec{g}$ además de acelerar el obstáculo, hace que se desarrollen inestabilidades de Rayleigh-Taylor (RT) en la parte de éste que se encuentra más cerca de la base del jet, como se muestra en la Figura 2.7 El tiempo en el cual estas inestabilidades crecen hasta una longitud de escala $l \sim R_{\mathrm{o}}$ está dado por

$$
t_{\mathrm{RT}} \sim \sqrt{\frac{l}{g}} \sim \sqrt{\frac{R_{\mathrm{o}}}{g}} \sim t_{\mathrm{co}} .
$$

Por otro lado, la diferencia relativa $v_{\text {rel }}$ entre la velocidad del material chocado del jet que rodea al obstáculo, $v_{\mathrm{j} 1}$, y la velocidad del material chocado del obstáculo, $v_{\mathrm{o} 1}$, desarrolla inestabilidades de Kelvin-Helmholtz $(\mathrm{KH})$, con una escala de tiempo dada por:

$$
t_{\mathrm{KH}} \sim \frac{l \sqrt{\chi}}{v_{\mathrm{rel}}} \sim \frac{R_{\mathrm{o}} \sqrt{\chi}}{v_{\mathrm{rel}}},
$$

donde nuevamente hemos considerado $l \sim R_{\mathrm{o}}$. Notar que como $v_{\mathrm{rel}} \sim v_{\mathrm{j} 1}, t_{\mathrm{KH}} \sim t_{\mathrm{co}}$. En las estimaciones de $t_{\mathrm{RT}} \mathrm{y}$ de $t_{\mathrm{KH}}$ no hemos tenido en cuenta al campo magnético, que podría estabilizar el sistema (Blake 1972, Romero 1995).

De las diferentes escalas de tiempo estimadas anteriormente, podemos resumir que una vez que el obstáculo penetra en el jet, el bow shock alcanza la distacia $Z$ rápidamente, en un tiempo $t_{\mathrm{bs}} \ll t_{\mathrm{co}}$ y luego el obstáculo podría ser destruido por las inestabilidades, acelerarse y comenzar a moverse conjuntamente con el jet o escapar de éste. Sin embargo, para hacer un análisis más exaustivo de cuales serán los procesos más relevantes necesitamos especificar la localización en el jet a la cual se produce la interacción. La determinación de este parámetro, conjuntamente con otras magnitudes del sistema (jet y obstáculo), serán expuestas en los Capítulos 5y 6, donde los escenarios considerados serán HMMQs y AGNs, respectivamente.

\subsubsection{Ondas de choque por interacciones globales}

Cuando un fluido supersónico choca con un medio externo, la onda de choque se disipa a medida que se propaga si la inyección de energía es puntual en el tiempo, como ocurre 

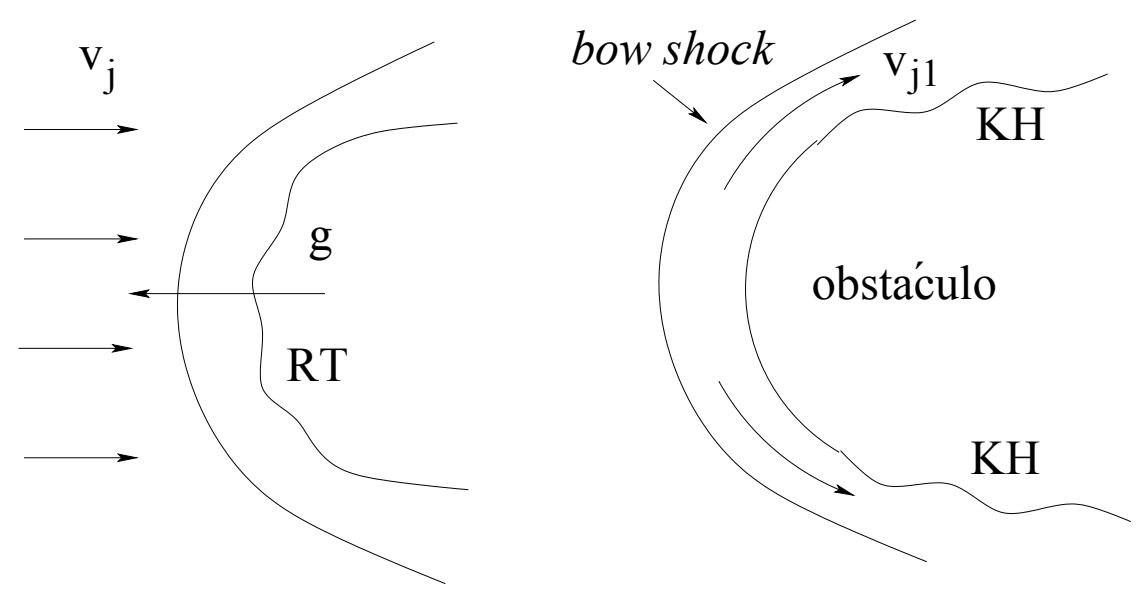

Figura 2.7: Diagrama del obstáculo y del bow shock. Las inestabilidades de RT se desarrollan en la superficie frontal del obstáculo (figura izquierda), mientras que las de KH lo hacen en las superficies laterales (figura derecha).

en las explosiones de supernovas. Por otro lado, si la inyección de energía es continua el choque se frena, pero más lentamente que en el caso anterior. Este choque llega a un estado estacionario en el caso radiativo. En las dos situaciones descriptas anteriormente se produce un choque reverso que se propaga en el sentido opuesto al movimiento del fluido. En el caso del evento puntual, el choque reverso al cabo de un cierto tiempo se disipará. Si la inyección inicial de energía es continua, se formará un choque estacionario.

Situaciones en las cuales pueden producirse choques de las maneras descriptas anteriormente pueden darse cuando los jets son frenados por el medio en el cual se propagan y se producen los llamados choques terminales, como ocurre por ejemplo en los jets de YSOs masivos y en los hot-spots de las radio-galaxias. Otra situación en la cual se desarrollan choques es cuando dos cúmulos de galaxias colisionan. Estos choques a gran escala se propagan hasta una cierta distancia del centro de gravedad de la fusión de ambos cúmulos y luego se disipan.

A diferencia de lo expuesto en la sección 2.5.1, aquí estudiaremos dos sistemas con propiedades muy diferentes: el jet de un YSO y un cúmulo de galaxias. Es por esto que la descripción de las escalas de tiempo que haremos a continuación será muy general y luego, en los capítulos correspondientes a cada fuente particular: YSOs (Capítulo 4) y cúmulos de galaxias (Capítulo 7), haremos un estudio más detallado de las escalas de tiempo con fórmulas pertinentes a cada fuente.

\section{Escalas de tiempo}

Supongamos un choque que se propaga a una velocidad $\vec{v}_{\text {ch }}$ en un medio externo. El choque, a medida que se propaga empuja y apila material delante de él, y así el frente de choque es ahora la "cabeza" de ese material que se mueve a la velocidad de la onda. Defini- 
mos el tiempo de propagación $t_{\mathrm{p}}$ como aquél en el cual el choque se frena sustancialmente, es decir, podemos suponer que durante $t_{\mathrm{p}}$ éste se propaga "libremente" por el medio una distancia $Z_{\mathrm{p}}$ en un tiempo:

$$
t_{\mathrm{p}} \sim \frac{Z_{\mathrm{p}}}{v_{\mathrm{ch}}}
$$

Sin embargo, mientras más material se haya acumulado, más dificil será moverlo y así la velocidad del choque va disminuyendo. Si la velocidad decrece, entonces la luminosidad también (de acuerdo a la ecuación (2.43)) y es así como el choque se va debilitando. Si $\vec{v}_{\text {ch }}$ disminuye, entonces hay fuertes variaciones de presión en la región chocada y se produce una onda. Si ésta se propaga a una velocidad mayor que $C_{\mathrm{s}}$ (ó $C_{\mathrm{sA}}$ ) entonces será una onda de choque y la llamaremos choque reverso. Este choque reverso se disipa o llega al estado estacionario si la inyección de energía es puntual o continua, respectivamente. En ambos casos, el choque reverso recorre una distancia $Z_{\mathrm{r}}$ a la velocidad $\vec{v}_{\mathrm{r}}$ en un tiempo

$$
t_{\mathrm{r}} \sim \frac{Z_{\mathrm{r}}}{v_{\mathrm{r}}}
$$

La velocidad $\vec{v}_{\text {r }}$ puede estimarse a través del contraste de densidades entre ambos medios chocados. Entre las regiones de ambos medios perturbados (el externo y el chocado por el choque reverso) se genera una superficie de discontinuidad, como se muestra en la Figura 2.8. En esta superficie, las inestabilidades de RT pueden ser importantes y mezclarse material de ambos medios chocados. La escala temporal de esta inestabilidad está determinada por la ecuación (2.51): $t_{\mathrm{RT}} \sim(l / g)^{1 / 2}$, pero considerando $l \sim Z_{\mathrm{r}}$ y que la aceleración es ejercida o bien por el material chocado del jet o bien por el centro del cúmulo de galaxias (en los casos particulares que estudiamos en esta tesis).

Finalmente, el choque reverso puede ser radiativo si $l_{\text {ter }}<Z_{\mathrm{r}}$, siendo $l_{\text {ter }} \sim t_{\text {ter }} v_{\mathrm{r}}$ y el tiempo de enfriamiento por emisión térmica

$$
t_{\text {ter }} \sim \frac{5}{2} \frac{P_{1}}{\mathcal{L}_{\text {ter }}}
$$

La función $\mathcal{L}_{\text {ter }}$ depende de la temperatura del medio chocado a través de la función $\Lambda(T)$, como se indica en la ecuación (2.29).

\subsection{Aceleración de partículas en choques}

Las ondas de choque, además de modificar las propiedades termodinámicas del medio por el cual se propagan, pueden también acelerar partículas hasta energías relativistas. Estas partículas aceleradas son llamadas partículas no térmicas, ya que su distribución en energía no es Maxweliana. El número de estas partículas no térmicas es una fracción pequeña del número de partículas termalizadas que forman el medio en el cual se propaga el choque. Es decir, el choque acelera sólo una fracción peque na de las partículas térmicas del medio sacándolas del estado de equilibrio termodinámico y convirtiéndolas en no térmicas. 


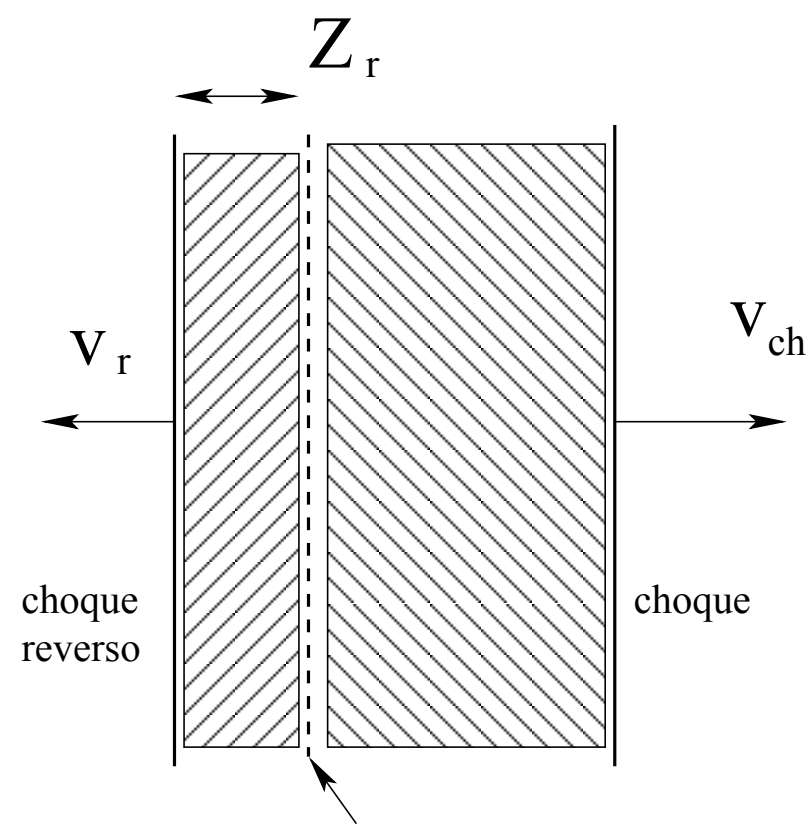

Superficie de discontinuidad

Figura 2.8: Esquema de un choque que se propaga en un medio externo con una velocidad $\vec{v}_{\text {ch }}$. Se forma un choque reverso que se propaga con una velocidad $\vec{v}_{\text {r }}$ en sentido opuesto a $\vec{v}_{\mathrm{ch}}$, y entre ambos medios chocados se forma una superficie de discontinuidad en la cual pueden desarrollarse inestabilidades de RT. 
La presencia de estas partículas en fuentes astrofísicas es usualmente detectada a través de la emisión no térmica en radio y de la radiación gamma (la cual no puede ser térmica).

El mecanismo propuesto originalmente por Fermi (1949) describe como las partículas podrían ser aceleradas por rebotes sucesivos entre centros dispersores con una distribución aleatoria de velocidades. Este proceso resulta poco eficiente ya que la ganancia de energía $E$ cada vez que la partícula rebota es $\langle\Delta E\rangle / E \propto \beta^{2}$, y como $\beta \ll 1$ la ganancia es muy poca ( $\beta c$ es la velocidad de la partícula). Por otro lado, además de ser poco eficiente, la situación en la cual tiene lugar el mecanismo es poco frecuente en la naturaleza. Sin embargo, es fácilmente adaptable a un escenario astrofísico si reemplazamos los centros dispersores por un choque e inhomogeneidades magnéticas en el medio. La teoría original de Fermi fue modificada en los años 70 por diversos autores, entre ellos Axford, Lear \& Skadron (1977) y Bell (1978) para describir un mecanismo más eficiente $(\langle\Delta E\rangle / E \propto \beta)$ y que tuviese lugar en sistemas astrofísicos con choques. Para que este proceso tenga lugar, es necesario que las partículas en el medio perturbado puedan difundir y alcanzar el choque. A continuación describiremos someramente el mecanismo de aceleración de Fermi modificado y que se conoce como mecanismo de Fermi de tipo I. Este proceso también se conoce como mecanismo de aceleración difusiva ya que la difusión juega un rol importante en el desarrollo del mismo.

En un plasma en el cual el campo magnético es uniforme, las partículas cargadas se mueven siguiendo trayectorias helicoidales alrededor de las líneas de $\vec{B}$. El radio de giro $\left(r_{\mathrm{g}}\right)$ está determinado por la energía $E$ de las partículas y por la intensidad del campo magnético:

$$
r_{\mathrm{g}}=\frac{E}{Z_{e} q_{e} B},
$$

donde $Z_{e}$ es el número atómicd y $q_{e}$ es la carga eléctrica del electrón. Sin embargo, en los sistemas astrofísicos que nos interesan el campo $\vec{B}$ presenta irregularidades que perturban el movimiento helicoidal de las partículas produciendo una reorientación de las mismas, y por lo tanto un movimiento desordenado. Las partículas con velocidad $\vec{v}$ difunden en el medio con un camino libre medio $\lambda=3 D / v$, donde $D$ es el coeficiente de difusión 5 . Si el movimiento de la partícula es en una dirección que forma un ángulo $\theta$ con $\vec{B}$, entonces $D=D_{\|} \cos ^{2}(\theta)+D_{\perp} \operatorname{sen}^{2}(\theta)$, siendo $D_{\|}$y $D_{\perp}$ los coeficientes de difusión en la dirección paralela y perpendicular a $\vec{B}$, respectivamente. Debido a que $D$ es desconocido en la mayoría de los sistemas astrofísicos, se suele considerar que $D_{\|}$es un número $\eta$ veces el coeficiente de difusión mínimo o de Bohm, $D_{\mathrm{B}}=r_{\mathrm{g}} c / 3$, y que significa considerar que el camino libre medio en la dirección paralela a $\vec{B}$ es $\lambda_{\|} \sim r_{\mathrm{g}}$. Luego resulta $D_{\perp} \approx D_{\|} /\left(1+\eta^{2}\right)$ (Jokipii 1987).

Con cada ciclo en el cual la partícula va del medio no chocado al chocado y vuelve al medio original, la ganancia de energía es $\langle\Delta E\rangle / E \sim(4 / 3) \beta$. Al cabo de $k_{\mathrm{c}}$ ciclos, la energía

\footnotetext{
${ }^{4}$ En todas las aplicaciones realizadas durante esta tesis $Z_{e}=1$.

${ }^{5}$ En el Capítulo 3 se dará la definición del camino libre medio.
} 
de la partícula, que inicialmente era $E_{i}$, resulta

$$
E=E_{i}\left(1+\frac{\Delta E}{E}\right)^{k_{\mathrm{c}}}
$$

El tiempo en el cual se realiza cada ciclo está determinado por como las partículas difunden en cada medio, es decir, de los coeficientes de difusión $D_{0}$ y $D_{1}$, a través de la expresión $t_{\text {ciclo }} \sim(4 / c)\left(D_{0} / v_{0}+D_{1} / v_{1}\right)$ (Protheroe 1999). El "tiempo de aceleración" es el tiempo requerido para que las partículas alcancen una energía $E$ a través de un mecanismo de aceleración dado; para el mecanismo de Fermi de tipo I resulta:

$$
t_{\mathrm{ac}} \equiv\left(\left.\frac{1}{E} \frac{\mathrm{d} E}{\mathrm{~d} t}\right|_{\mathrm{ac}}\right)^{-1}=\frac{E}{\langle\Delta E\rangle} t_{\text {ciclo }} \sim \frac{3 \zeta}{(\zeta-1) v_{0}}\left(\frac{D_{0}}{v_{0}}+\frac{D_{1}}{v_{1}}\right) .
$$

Así, el tiempo de aceleración depende de la geometría de $\vec{B}$. A continuación damos expresiones sencillas para $t_{\text {ac }}$ correspondientes a configuraciones particulares de $\vec{B}$.

- Choques paralelos $\left(\vec{B} \| \vec{v}_{\mathrm{ch}}\right)$ : suponiendo que $D_{0}=D_{1}=D_{\|}$y que $\vec{B}_{0}=\vec{B}_{1}$ se tiene que el tiempo de aceleración es

$$
t_{\mathrm{ac}}^{\|} \sim \frac{20}{3} \frac{\eta E}{q_{e} B_{0} v_{\mathrm{ch}}^{2}}
$$

- Choques transversales $\left(\vec{B} \perp \vec{v}_{\mathrm{ch}}\right)$ : suponiendo que $D_{0}=D_{1}=D_{\perp}$ y que $\vec{B}_{1} \sim 4 \vec{B}_{0}$ se tiene que el tiempo de aceleración es

$$
t_{\mathrm{ac}}^{\perp} \sim \frac{8}{3} \frac{E}{\eta q_{e} B_{0} v_{\mathrm{ch}}^{2}} .
$$

Por otro lado, debido a que en los choques relativistas el cálculo de $D$ es aún más complicado que en los NR, se puede considerar que en los primeros $t_{\mathrm{ac}} \sim E /\left(0.1 q_{e} B_{0} c\right.$ ) (como en el caso de la supernova del Cangrejo - $C r a b-)$, sin especificar la geometría de $\vec{B}$.

El número de ciclos que realiza la partícula depende básicamente del tamaño $L_{\mathrm{ac}}$ del acelerador. Las partículas pueden cruzar el choque sucesivas veces antes de que $r_{\mathrm{g}}(\propto E)$ crezca lo suficiente, es decir, $r_{\mathrm{g}} \sim L_{\mathrm{ac}}$, como para escapar del acelerador (Hillas 1984). Al cabo de $k_{\mathrm{c}}$ ciclos el espectro de las partículas aceleradas e inyectadas en el medio chocado será $Q(E)=K_{0} E^{-p}$, donde

$$
p=\frac{\ln \left(E / E_{i}\right)}{\ln (1+\Delta E / E)}
$$

$\mathrm{y}[Q]=\operatorname{erg}^{-1} \mathrm{~s}^{-1}$. En la ecuación anterior la dependencia con $k_{\mathrm{c}}$ está implícita en $E / E_{i}$. Esta inyección $Q(E)$ de partículas por unidad de tiempo en una determinada región de la fuente dará lugar, al cabo de un tiempo $t$, a una distribución $N(E, t)$ de partículas no térmicas. 


\subsubsection{Población de partículas no térmicas}

Dada una distribución $Q(E)$ de partículas aceleradas e inyectadas en una región, nos interesa conocer cual será la distribución de partículas $N(E, t)$ en un tiempo $t$.

A lo largo de esta tesis consideraremos que tanto la región donde se aceleran las partículas, el acelerador, como aquella en la cual radían, el emisor, son homogénea:6. Es decir, las magnitudes físicas no dependerán de las coordenadas espaciales. Bajo esta hipótesis, la ecuación que describe la evolución temporal de $N(E, t)$ es la siguiente (Ginzburg \& Syrovatskii, 1964):

$$
\frac{\partial N(E, t)}{\partial t}=\frac{\partial}{\partial E}\left(\left.\frac{\mathrm{d} E}{\mathrm{~d} t}\right|_{\mathrm{rad}} N(E, t)\right)-\frac{N(E, t)}{t_{\mathrm{esc}}}+Q(E)
$$

donde $[N(E, t)]=\mathrm{erg}^{-1}$. Esta ecuación tiene en cuenta la inyección de partículas $Q(E)$ que en un tiempo $t$ nos da la distribución $N(E, t)$. Las partículas desde que se inyectaron en el tiempo $t_{0}=0$ hasta el tiempo $t$ sufren pérdidas radiativas que están contempladas en el término $\mathrm{d} E /\left.\mathrm{d} t\right|_{\mathrm{rad}} \equiv \dot{E}_{\mathrm{rad}}$. Además, puede haber pérdidas de energía en el sistema debido a que las partículas pueden escapar del acelerador. El escape de las partículas está considerado en el término $N(E, t) / t_{\text {esc }}$, donde

$$
t_{\mathrm{esc}}^{-1}=\left(\frac{1}{t_{\mathrm{conv}}}+\frac{1}{t_{\mathrm{dif}}}\right)
$$

Esta escala de tiempo tiene en cuenta el escape por convección $\left(t_{\text {conv }}\right)$ y por difusión $\left(t_{\text {dif }}\right)$. Mientras que el primero contempla el arrastre de las partículas por el movimiento del medio chocado (que se mueve a una velocidad $\sim v_{\mathrm{ch}} / 4$ ), el segundo considera las pérdidas de energía por difusión de las partículas.

La solución más general de la ecuación (2.62) considerando que $t_{\mathrm{esc}}=t_{\mathrm{conv}}$, es decir, $t_{\text {conv }} \ll t_{\text {diff }}$, es (Khangulyan et al. 2007)

$$
N(E, t)=\frac{1}{|\dot{E}|} \int_{E}^{E_{\mathrm{eff}}} Q\left(t, E^{\prime}\right) \exp \left(-\frac{\tau\left(E, E^{\prime}\right)}{t_{\mathrm{esc}}}\right) \mathrm{d} E^{\prime},
$$

donde $E_{\text {eff }}$ es la energía máxima de las partículas que en el tiempo $t$ pueden enfriarse hasta una energía $E$, es decir,

$$
t(E)=\int_{E}^{E_{\text {eff }}} \frac{\mathrm{d} E^{\prime}}{\left|\dot{E}^{\prime}\right|} \quad \text { y } \quad \tau\left(E, E^{\prime}\right)=\int_{E}^{E^{\prime}} \frac{\mathrm{d} E^{\prime \prime}}{\left|\dot{E}^{\prime \prime}\right|} .
$$

Dada una energía $E^{\prime}, \tau$ es el tiempo en el cual una partícula de energía $E^{\prime}$ se enfría hasta obtener una energía $E$. Notemos que $\tau \leq t$. La ecuación (2.64) toma una expresión muy sencilla en dos casos límites que veremos a continuación, si $p>1$ :

\footnotetext{
${ }^{6}$ Notamos que el campo magnético debe tener una componente inhomogénea para que se el mecanismo de Fermi pueda desarrollarse.
} 
- Si $t(E) \ll t_{\text {esc }}$ entonces $\tau\left(E, E^{\prime}\right) \ll t_{\text {esc }}$ y entonces el término $\exp \left(-\tau / t_{\text {esc }}\right) \sim 1$, con lo cual $N(E, t) \sim\left(-Q(E)+Q\left(E_{\text {eff }}\right)\right)\left(E_{\text {eff }}-E\right) /|\dot{E}| \sim Q(E) t(E)$ y las partículas se acumulan a medida que $t$ crece. Este proceso de acumulación de partículas de energía $E$ continúa hasta que $E_{\text {eff }}$ coincide con la energía máxima de la distribución $Q(E)$ o el tiempo de escape se hace menor que el tiempo de enfriamiento.

- Si $t_{\text {esc }} \ll t(E): N(E) \sim Q(E) t_{\text {esc }}$ y el sistema llega a un estado estacionario.

Para conocer la distribución $N(E)$ necesitamos conocer las pérdidas radiativas que sufren las partículas. Para esto, en el próximo capítulo repasaremos los procesos radiativos que hemos estudiado durante esta tesis. 


\section{Capítulo 3}

\section{Procesos radiativos}

Todo lo que conocemos de la mayoría de las fuentes astrofísicas es gracias a que ellas radían y a que es posible detectar parte de los fotones emitidos con instrumentos adecuados. Por esta razón, conocer los procesos a través de los cuales se genera la emisión es indispensable para modelizar correctamente las fuentes que nos interesa estudiar.

Como mencionamos en el Capítulo 1, la emisión de rayos gamma no es posible mediante procesos en equilibrio termodinámico. La producción de estos fotones tan energéticos ocurre a través de procesos no térmicos, esto es, por la interacción de partículas relativistas con campos de materia, de fotones y magnéticos. Todas estas interacciones pueden describirse como dispersiones de las partículas relativistas por la interacción con otras partículas (en ocaciones virtuales) y que como resultado producen fotones. Por esto, para estudiar la producción de radiación (gamma, en particular), es conveniente definir algunos conceptos básicos que son comunes a todos los procesos radiativos no térmicos y que usaremos a lo largo de toda la tesis.

\subsection{Conceptos básicos}

Dada una población de partículas de tipo $i$ contenidas en un volumen $V$, si todas ellas tienen energías $E_{i}$ diferentes tales que $E_{i}^{\min } \leq E_{i} \leq E_{i}^{\max }$, la densidad total de estas particulas será

$$
n_{i}=\int_{E_{i}^{\min }}^{E_{i}^{\max }} \frac{\mathrm{d} n_{i}}{\mathrm{~d} E_{i}} \mathrm{~d} E_{i},
$$

donde $\left[n_{i}\right]=\mathrm{cm}^{-3}$. La densidad de energía de estas partículas es

$$
u_{i}=\int_{E_{i}^{\min }}^{E_{i}^{\max }} \frac{\mathrm{d} n_{i}}{\mathrm{~d} E_{i}} E_{i} \mathrm{~d} E_{i},
$$

siendo $\left[u_{i}\right]=\mathrm{erg} \mathrm{cm}^{-3}$. Si la distribución fuese monoenergética, es decir, $E_{i}$ constante, entonces $u_{i}=n_{i} E_{i}$. Si la velocidad de las partículas es $v_{i}$, la luminosidad de éstas será

$$
L_{i}=u_{i} v_{i} S,
$$


donde la superficie $S=\partial V$ y $\left[L_{i}\right]=\mathrm{erg} \mathrm{s}^{-1}$. Si las partículas son relativistas, con una energía cinética $\left(\Gamma_{i}-1\right) m_{i} c^{2}$, la ecuación (3.3) se escribe como (2.43).

Supongamos que inyectamos una partícula relativista, con energía $E_{i}$, en un medio en el cual existe una densidad $n_{\mathrm{b}}$ de partículas que llamaremos blanco y cuya energía es $E_{\mathrm{b}}$. En el SR de la partícula relativista, las partículas del medio se mueven a una velocidad $\sim c$ y el número de interacciones que ocurren por unidad de tiempo es

$$
N_{\mathrm{int}}=\sigma\left(E_{i}\right) n_{\mathrm{b}} c
$$

donde $\sigma\left(E_{i}\right)$ es una medida de la superficie efectiva $\left([\sigma]=\mathrm{cm}^{2}\right)$ de interacción del proceso de dispersión y conocida como sección eficazl. Mientras mayor sea $\sigma$, mayor es la probabilidad de dispersión. A la energía de los fotones creados en la interacción la llamaremos $E_{\mathrm{ph}}$ y depende de la energía inicial $E_{\mathrm{b}}$, de la energía de la partícula relativista, $E_{i}$, y del ángulo de interacción. Sin embargo, $E_{\text {ph }}$ no está completamente determinada ya que depende de las características de la colisión. Por lo tanto, el estado final, caracterizado por $E_{\text {ph }}$ y por el ángulo de dispersión $\Omega$, tendrá asociada una función de probabilidad. Esta información está contenida en la sección eficaz diferencial $\mathrm{d} \sigma\left(E_{\mathrm{ph}}, E_{i}, \Omega\right) / \mathrm{d} E_{\mathrm{ph}} \mathrm{d} \Omega$, de tal manera que la sección eficaz total es

$$
\sigma\left(E_{i}\right)=\int \frac{\mathrm{d}^{2} \sigma\left(E_{\mathrm{ph}}, E_{i}, \Omega\right)}{\mathrm{d} E_{\mathrm{ph}} \mathrm{d} \Omega} \mathrm{d} E_{\mathrm{ph}} \mathrm{d} \Omega .
$$

Debido a la naturaleza del proceso, la distribución energética de los fotones creados o dispersados dependerá fuertemente del ángulo de interacción. Sin embargo, ya que en la mayoría de las situaciones astrofísicas este ángulo no se conoce, se suelen tomar las magnitudes isotropizadas, es decir, integradas en todos los posibles ángulos de interacción (o de dispersión). En esta tesis no consideraremos las dependencias angulares (ni espaciales: emisor homogéneo) de las magnitudes. De la ecuación (3.4), si $x=c t=\left(\sigma n_{\mathrm{b}}\right)^{-1}$, entonces se tiene que ocurre solo una interacción en el tiempo $t$. Se define el camino libre medio como la distancia $\lambda$ que las partículas recorren entre dos interacciones sucesivas:

$$
\lambda \equiv \frac{1}{\sigma\left(E_{i}\right) n_{\mathrm{b}}}
$$

El tiempo de enfriamiento $t_{\mathrm{rad}}$ es una medida de la eficiencia de un proceso de interacción de una partícula relativista con un blanco de densidad $n_{\mathrm{b}}$. Si en este proceso la partícula relativista pierde una cantidad de energía $E_{i}-E_{f}$, donde $E_{i}$ y $E_{f}$ son las energías inicial y final, respectivamente, y definimos la inelasticidad $f$ del proceso como $f=\left(E_{i}-E_{f}\right) / E_{i}$, entonces

$$
t_{\mathrm{rad}}=\frac{1}{f \sigma\left(E_{i}\right) n_{\mathrm{b}} c} .
$$

\footnotetext{
${ }^{1}$ Debido a que los valores de $\sigma$ son muy chicos, la unidad de medida que se suele usar es el Barn: 1b $=10^{-24} \mathrm{~cm}^{2}$.
} 
Las pérdidas de energía que mencionamos en el capítulo anterior, en la ecuación (2.62), se definen ahora como

$$
\left.\frac{\mathrm{d} E_{i}}{\mathrm{~d} t}\right|_{\mathrm{rad}} \equiv-\frac{E_{i}}{t_{\mathrm{rad}}}
$$

Supongamos que inyectamos una distribución $Q_{i}\left(E_{i}\right)$ de partículas relativistas en el medio. Al cabo de un tiempo $\geq t_{\text {rad/esc }}$, la distribución de éstas será $N_{i}\left(E_{i}\right) \sim Q_{i}\left(E_{i}\right) t_{\text {rad/esc }}$. El número de éstas partículas acumuladas en el volumen $V$ serå2:

$$
N_{i}^{\mathrm{rel}}=\int_{E_{i}^{\min }}^{E_{i}^{\max }} N_{i}\left(E_{i}\right) \mathrm{d} E_{i}
$$

Para conocer el espectro de fotones producidos en la interacción de las $N_{i}^{\text {rel }}$ partículas relativistas y cuya distribución en energías es $N_{i}\left(E_{i}\right)$, necesitamos conocer la emisividad $J_{\text {ph }}$ de la fuente. Si las partículas son relativistas podemos suponer que se mueven a una velocidad $\sim c$ y entonces

$$
J_{\mathrm{ph}}\left(E_{\mathrm{ph}}\right) \sim \frac{c}{4 \pi} \int_{E_{i}=E_{\mathrm{ph}}}^{E_{i}^{\max }} n_{\mathrm{b}} \frac{\mathrm{d} \sigma\left(E_{\mathrm{ph}}, E_{i}\right)}{\mathrm{d} E_{\mathrm{ph}}} N_{i}\left(E_{i}\right) \mathrm{d} E_{i},
$$

donde $\left[J_{\mathrm{ph}}\right]=\operatorname{erg}^{-1} \mathrm{~s}^{-1}$. Si conocemos $J_{\mathrm{ph}}$ luego la luminosidad específica en el caso de un emisor homogéneo e isotrópico es

$$
L_{E_{\mathrm{ph}}} \sim E_{\mathrm{ph}} J_{\mathrm{ph}}
$$

$\mathrm{y}\left[L_{E_{\mathrm{ph}}}\right]=\mathrm{s}^{-1}$.

A continuación haremos una somera descripción de los cuatro procesos radiativos que hemos considerado en esta tesis: radiación sincrotrón, dispersión Compton inversa (IC), Bremsstrahlung relativista e interacciones protón-protón $(p p)$.

\subsection{Procesos radiativos}

Existen varias maneras de producir fotones. Una de ellas es a través de la aceleración de partículas cargadas a través de la fuerza ejercida sobre ellas por algún campo. Otra manera es a través del decaimiento de partículas.

\subsubsection{Radiación sincrotrón}

Las partículas cargadas sienten la fuerza de Lorentz que ejerce sobre ellas el campo electromagnético. Esta fuerza hace que las partículas describan un movimiento helicoidal

\footnotetext{
${ }^{2} \mathrm{~A}$ las magnitudes intensivas las llamamos con letras minúsculas y a las extensivas con mayúsculas. Por ejemplo, las distribuciones de energía de las partículas serán $n_{i}\left(E_{i}\right)$ si son por unidad de volumen y $N_{i}\left(E_{i}\right)$ en caso contrario. De esta manera, bajo la suposición de que $n_{i}\left(E_{i}\right)$ no depende de las coordenadas espaciales, $N_{i}\left(E_{i}\right)=n_{i}\left(E_{i}\right) V$.
} 


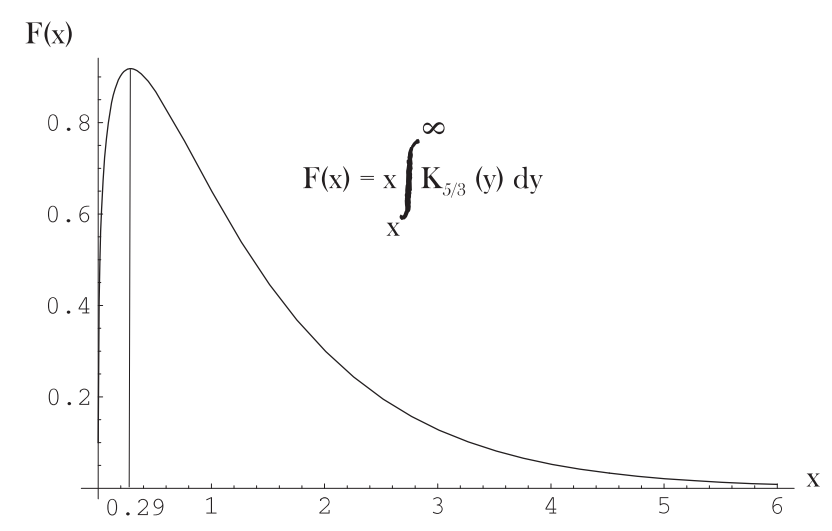

Figura 3.1: Gráfico de la función $F(x)$, donde $x=E_{\mathrm{ph}} / E_{\mathrm{c}}$. Como puede apreciarse, el máximo corresponde a $x=0.3$, esto es, $E_{\mathrm{ph}}=0.3 E_{\mathrm{c}}$. Gracias a esta forma tipo función $\delta$ que muestra $P_{\sin }\left(E_{\mathrm{ph}}, E_{e}\right)$ es posible adoptar la aproximación de que los electrones de energía $E_{e}$ emiten fotones de energía $\sim 0.3 E_{\mathrm{c}}\left(E_{e}\right)$.

alrededor de las líneas de campo magnético $\vec{B}$. Las partículas relativistas que son aceleradas por la fuerza de Lorentz producen la llamada radiación sincrotrón. Como veremos luego, este proceso radiativo es mucho más eficiente para leptones que para hadrones, por lo cual en esta tesis sólo consideraremos la radiación sincrotrón producida por los primeros. Otra característica de este proceso es que los fotones son emitidos en una dirección preferencial, aquella correspondiente al movimiento de la partícula.

La radiación es emitida en un cono cuyo ángulo de apertura llamaremos $\theta \sim 1 / \Gamma_{e}$, donde $\Gamma_{e}=E_{e} /\left(m_{e} c^{2}\right)$ es el factor de Lorentz de un electrón con energía $E_{e}$. La distribución en energía de la potencia sincrotrón por partícula es

$$
P_{\mathrm{sin}}\left(E_{e}, E_{\mathrm{ph}}\right)=\frac{1}{h} \frac{\sqrt{3} e^{3}}{m_{e} c^{2}} B_{\perp} \frac{E_{\mathrm{ph}}}{E_{\mathrm{c}}} \int_{E_{\mathrm{ph}} / E_{\mathrm{c}}}^{\infty} K_{5 / 3}(\zeta) \mathrm{d} \zeta
$$

donde $B_{\perp}$ es la componente de $\vec{B}$ perpendicular a la dirección del movimiento de la partícula. Si asumimos que $\vec{B}$ es isotrópico, entonces $B_{\perp}=\sqrt{2 / 3} B$. La energía característica de los fotones producidos es $E_{\mathrm{c}}\left(E_{e}\right)=5.1 \times 10^{-8} B E_{e}^{2}$ erg. La función de Bessel de segunda especie y de orden $5 / 3, K_{5 / 3}$, tiene una forma tal que la integral de ella por el cociente $E_{e} / E_{c}$ puede aproximarse de la siguiente manera

$$
\frac{E_{\mathrm{ph}}}{E_{\mathrm{c}}} \int_{E_{\mathrm{ph}} / E_{\mathrm{c}}}^{\infty} K_{5 / 3}(\zeta) \mathrm{d} \zeta \sim 1.85\left(\frac{E_{\mathrm{ph}}}{E_{\mathrm{c}}}\right)^{1 / 3} \exp \left(\frac{-E_{\mathrm{ph}}}{E_{\mathrm{c}}}\right) .
$$

La función $P_{\sin }\left(E_{e}, E_{\mathrm{ph}}\right)$ tiene un máximo muy pronunciado en la energía $E_{\mathrm{ph}} \sim 0.3 E_{\mathrm{c}}$, como se muestra en la Figura 3.1 .

La pérdida total de energía radiada por un electrón de energía $E_{e}$ se obtiene integrando 
la distribución (3.12) en todas las energías radiadas $E_{\mathrm{ph}}$ :

$$
\left.\frac{\mathrm{d} E_{e}}{\mathrm{~d} t}\right|_{\sin } \equiv-P_{\sin }\left(E_{e}\right)=-\int P_{\sin }\left(E_{e}, E_{\mathrm{ph}}\right) \mathrm{d} E_{\mathrm{ph}} .
$$

Luego, el tiempo de enfriamiento resulta

$$
t_{\mathrm{sin}}=\frac{2 \pi}{3 c \sigma_{\mathrm{T}}} \frac{\left(m_{e} c^{2}\right)^{2}}{E_{e} B^{2}} \sim \frac{4.1 \times 10^{2}}{B^{2} E_{e}} \mathrm{~s} .
$$

Siendo $t_{\sin } \propto m^{4}$, donde $m$ es la masa de la partícula que está radiando, el tiempo de enfriamiento por radiación sincrotrón será $\sim 10^{12}$ veces más corto para electrones que para protones, ya que $m_{e} \sim 10^{-3} m_{p}$.

Si ahora consideramos que inyectamos una población $Q_{e}\left(E_{e}\right) \propto E_{e}^{-p}$ de electrones relativistas, las pérdidas que sufrirán por radiación sincrotrón modificarán $Q_{e}\left(E_{e}\right)$ dando lugar a la distribución de electrones

$$
N_{e}\left(E_{e}\right) \sim Q_{e}\left(E_{e}\right) t_{\sin } \propto E_{e}^{-p-1}
$$

Dada la distribución $N_{e}\left(E_{e}\right)$, para obtener la potencia radiada por todos estos electrones lo que debemos hacer es integrar el siguiente producto:

$$
P_{\mathrm{sin}}\left(E_{\mathrm{ph}}\right)=\int_{E_{e}^{\min }}^{E_{e}^{\max }} P\left(E_{e}, E_{\mathrm{ph}}\right) N_{e}\left(E_{e}\right) \mathrm{d} E_{e},
$$

donde $E_{e}^{\min }$ y $E_{e}^{\max }$ son las energías mínima y máxima, respectivamente, de la distribución de electrones relativistas. Sin embargo, debido a que cada electrón radiará la mayor parte de su energía $E_{e}$ en fotones de energía $E_{\mathrm{ph}} \sim 0.3 E_{\mathrm{c}}\left(E_{e}\right)$, podemos calcular la integral (3.17) considerando una aproximación tipo $\delta$. De esta manera, utilizando una aproximación $\delta\left(E_{\mathrm{ph}}-0.3 E_{\mathrm{c}}\right)$ podemos hallar una solución analítica aproximada de $P_{\sin }\left(E_{\mathrm{ph}}\right)$.

Para obtener la luminosidad a la energía $E_{\mathrm{ph}}$ calculamos $E_{\mathrm{ph}} L_{\mathrm{ph}}=E_{\mathrm{ph}} P_{\mathrm{sin}}\left(E_{\mathrm{ph}}\right)$. Es interesante notar que el espectro de fotones emitidos tiene una distribución en energía tipo ley de potencias de índice $\alpha\left(E_{\mathrm{ph}} L_{\mathrm{sin}} \propto E_{\mathrm{ph}}^{-\alpha}\right)$, si el espectro de electrones también es una ley de potencias $N_{e} \propto E_{e}^{-p^{\prime}}$. Las relaciones entre ambos índices es $\alpha=\left(p^{\prime}-1\right) / 2$.

\subsubsection{Radiación Compton inversa}

Consideremos un gas de fotones de energía $E_{\text {phm }}$ cuya densidad de energía es $u_{\mathrm{phm}} \mathrm{y}$ un electrón relativista que atraviesa dicho gas. Si en el SR del laboratorio los fotones son menos energéticos que el electrón, entonces los primeros serán dispersados por el segundo. Como producto de esta interacción los fotones ganan energía, a diferencia de lo que ocure en la interacciones Compton (directas), y de aquí que se las llame dispersiones Compton inversas.

En el SR del electrón, si se acerca un fotón poco energético, éste sufrirá un cambio mayor en su energía y momento que uno con más energía. Esto se refleja en la sección 
eficaz de la interacción, $\sigma_{\mathrm{IC}}$. Si integramos en todos los posibles ángulos de incidencia, $\sigma_{\mathrm{IC}}$ depende de la energía de los fotones semilla y del electrón a través de la expresión (Vila \& Aharonian 2009)

$$
\sigma_{\mathrm{IC}}=\frac{3 \sigma_{\mathrm{T}}}{8 y}\left[\left(1-\frac{2}{y}-\frac{2}{y^{2}}\right) \ln (1+2 y)+\frac{1}{2}+\frac{8}{y}-\frac{1}{2(1+2 y)^{2}}\right],
$$

donde $y \equiv E_{\mathrm{phm}} E_{e} /\left(m_{e}^{2} c^{4}\right)$ y $\sigma_{\mathrm{T}} \sim 0.67$ b es la sección eficaz de Thomson. Notamos que para $y \ll 1, \sigma_{\mathrm{IC}} \sim \sigma_{\mathrm{T}} \mathrm{y}$ se dice que la interacción ocurre en el régimen de Thomson (Th), mientras que si $y \gg 1$ entonces $\sigma_{\mathrm{IC}} \sim\left(3 \sigma_{\mathrm{T}} / 8\right) \ln (4 y) / y$ y se dice que interacción se desarrolla en el régimen de Klein-Nishina (KN). Como se muestra en la Figura 3.2 . en el régimen de KN $\sigma_{\mathrm{IC}}$ cae abruptamente. Sin embargo, las pérdidas de energía por interacción son mayores en el régimen KN que en el Th, siendo en el primero catastróficas, esto es, $E_{\mathrm{ph}} \sim E_{e}$. En el régimen Th, la energía máxima que pueden alcanzar los fotones es $E_{\mathrm{ph}}^{\max } \sim(4 / 3) \Gamma_{e}^{2} E_{\mathrm{phm}}$.

Dado un electrón relativista inmerso en un gas de fotones, el número de interacciones por unidad de tiempo puede calcularse a través de la ecuación (3.4), considerando que $n_{\mathrm{b}}=n_{\mathrm{phm}}$, con lo cual obtenemos que el número de interacciones por unidad de tiempo es $N_{\text {int }}=n_{\text {phm }} \sigma_{\text {IC }} c$. El número de interacciones es igual al número de fotones dispersados. $\mathrm{Si}$ ahora queremos conocer la distribución energética de los fotones dispersados por un electrón, debemos tener en cuenta que la energía $E_{\mathrm{ph}}$ de estos fotones no está fija sino que es una distribución y esto se muestra en la sección eficaz diferencial (Blumenthal \& Gould, 1970)

$$
\frac{\mathrm{d} \sigma_{\mathrm{IC}}}{\mathrm{d} E_{\mathrm{ph}}}\left(E_{\mathrm{ph}}, E_{e}\right)=\frac{3 \sigma_{\mathrm{T}}}{4 E_{\mathrm{ph}} \Gamma_{e}^{2}} f\left(x_{\mathrm{ic}}\right)
$$

con

$$
f\left(x_{\mathrm{ic}}\right)=\left[2 x_{\mathrm{ic}} \ln x_{\mathrm{ic}}+x_{\mathrm{ic}}+1-2 x_{\mathrm{ic}}^{2}+\frac{\left(4 \epsilon_{\mathrm{ph}} \Gamma_{e} x_{\mathrm{ic}}\right)^{2}\left(1-x_{\mathrm{ic}}\right)}{2\left(1+4 \epsilon_{\mathrm{ph}} \Gamma_{e} x_{\mathrm{ic}}\right)}\right] P_{\mathrm{ic}}\left(\frac{1}{4 \Gamma_{e}^{2}}, 1, x_{\mathrm{ic}}\right),
$$

donde $\epsilon_{\mathrm{ph}}=E_{\mathrm{ph}} / m_{e} c^{2}$. La función $P_{\mathrm{ic}}$ es igual a 1 cuando $1 / 4 \Gamma_{e}^{2} \leq x_{\mathrm{ic}} \leq 1$ y $P_{\mathrm{ic}}=0$ en cualquier otro caso. Siendo $\epsilon_{\mathrm{phm}}=E_{\mathrm{phm}} / m_{e} c^{2}$, el parámetro adimensional $x_{\mathrm{ic}}$ se define de la siguiente manera

$$
x_{\mathrm{ic}}=\frac{\epsilon_{\mathrm{ph}}}{4 \epsilon_{\mathrm{phm}} \Gamma_{e}^{2}\left(1-\epsilon_{\mathrm{ph}} / \Gamma_{e}\right)} .
$$

La interacción de un electrón con un campo de fotones dispersa a éstos dando un espectro de fotones dispersados con energías $E_{\mathrm{ph}}^{\mathrm{min}} \leq E_{\mathrm{ph}} \leq E_{\mathrm{ph}}^{\max }$. Para calcular la potencia radiada por un electrón por interacciones IC debemos integrar sobre todas las energías $E_{\mathrm{phm}}$ a través de la expresión

$$
P_{\mathrm{IC}}\left(E_{e}\right)=-\left.\frac{\mathrm{d} E}{\mathrm{~d} t}\right|_{\mathrm{IC}}=c \int_{E_{\mathrm{phm}}^{\min }}^{E_{\mathrm{phm}}^{\max }} \int_{E_{\mathrm{ph}}^{\min }}^{E_{\mathrm{ph}}^{\max }} \frac{\mathrm{d} \sigma_{\mathrm{IC}}\left(E_{\mathrm{ph}}, E_{e}\right)}{\mathrm{d} E_{\mathrm{ph}}} \frac{\mathrm{d} n_{\mathrm{phm}}\left(E_{\mathrm{phm}}\right)}{\mathrm{d} E_{\mathrm{phm}}} E_{\mathrm{ph}} \mathrm{d} E_{\mathrm{phm}} \mathrm{d} E_{\mathrm{ph}} .
$$




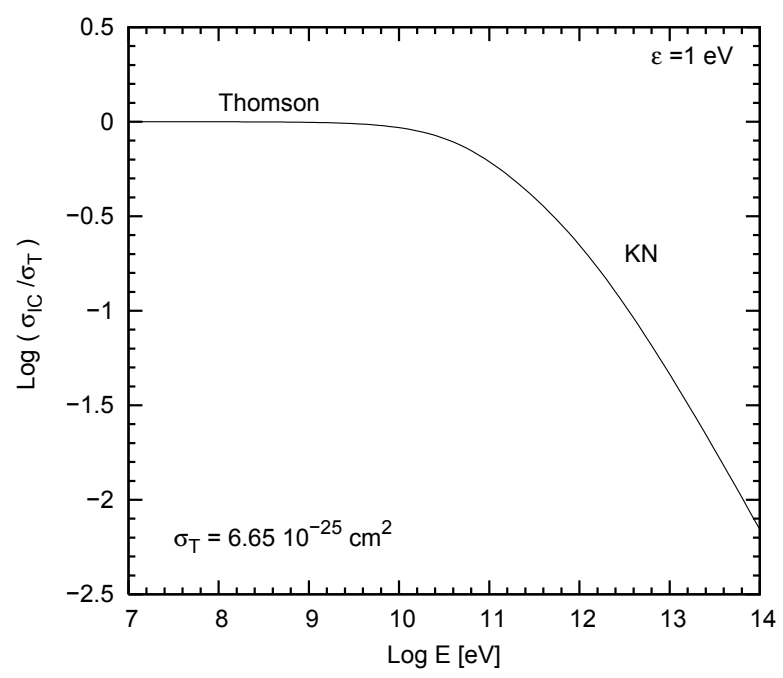

Figura 3.2: Sección eficaz de la interacción IC en los regímenes Th y KN para fotones semilla con energía $\tilde{E}_{\mathrm{phm}}=1 \mathrm{eV}$.

Para el caso en el cual los fotones semilla siguen una distribución (quasi)monoenergética con energía $\tilde{E}_{\mathrm{phm}}$, las pérdidas de un electrón pueden obtenerse a través de una aproximación $\delta\left(E_{\mathrm{phm}}-\tilde{E}_{\mathrm{phm}}\right)$. El tiempo de enfriamiento tanto en el régimen de Th como en el de KN queda determinado por la expresión (Bosch-Ramon \& Khangulyan, 2009)

$$
t_{\mathrm{IC}}=\frac{6.1 \times 10^{12} \tilde{E}_{\mathrm{phm}}}{u_{\mathrm{phm}}} \frac{(1+8.3 y)}{\ln (1+0.2 y)} \frac{\left(1+1.3 y^{2}\right)}{\left(1+0.5 y+1.3 y^{2}\right)} \mathrm{s},
$$

donde ahora $y=\tilde{E}_{\mathrm{phm}} E_{e} /\left(m_{e}^{2} c^{4}\right)$ y $u_{\mathrm{phm}}$ es la densidad de energía de los fotones ambientales. Considerando los límites a bajas energías de (3.23), es posible hallar $t_{\mathrm{IC}}$ en el régimen de Th, resultando

$$
t_{\mathrm{IC}}^{\mathrm{Th}} \sim \frac{24}{E_{e} u_{\mathrm{phm}}} \mathrm{s} .
$$

Si ahora inyectamos una distribución $Q_{e}\left(E_{e}\right) \propto E_{e}^{-p}$ de electrones relativistas en el gas de fotones, las pérdidas por IC en el régimen Th modifican el espectro de la misma manera que las pérdidas por radiación sincrotrón, esto es,

$$
N_{e}\left(E_{e}\right) \sim Q_{e}\left(E_{e}\right) t_{\mathrm{IC}}^{\mathrm{Th}} \propto E_{e}^{-p-1} .
$$

Dada la distribución $N_{e}\left(E_{e}\right)$, para obtener la distribución de fotones dispersados calculamos la emisividad de los mismos a través de

$$
J_{\mathrm{IC}}\left(E_{\mathrm{ph}}\right)=\frac{c}{4 \pi} \int_{E_{e}^{\min }}^{E_{e}^{\max }} \int_{E_{\mathrm{phm}}^{\min }}^{E_{\mathrm{phm}}^{\max }} \frac{\mathrm{d} \sigma_{\mathrm{IC}}\left(E_{\mathrm{ph}}, E_{e}\right)}{\mathrm{d} E_{\mathrm{ph}}} \frac{\mathrm{d} n_{\mathrm{phm}}\left(E_{\mathrm{phm}}\right)}{\mathrm{d} E_{\mathrm{phm}}} N_{e}\left(E_{e}\right) \mathrm{d} E_{\mathrm{phm}} \mathrm{d} E_{e} .
$$




\section{Auto Compton}

Si los fotones semilla son creados externamente a la fuente (es decir, fuera del volumen V) entonces el proceso se dice Compton externo (EC, por External Compton). Por otro lado, en el caso particular de que los fotones semilla sean producidos por radiación sincrotrón de la misma población de electrones $N_{e}\left(E_{e}\right)$ que interactúan por IC, entonces el proceso se llama auto Compton sincrotrón (SSC, por Synchrotron Self Compton). Bajo esta situación, la distribución de fotones semilla es una ley de potencias y para calcular el espectro de los fotones producidos debemos usar la ecuación (3.26), considerando que $\mathrm{d} n_{\mathrm{phm}} / \mathrm{d} E_{\mathrm{phm}}=$ $\mathrm{d} n_{\sin } / \mathrm{d} E_{\sin }$ es la densidad de fotones producidos por radiación sincrotrón.

\subsubsection{Bremsstrahlung relativista}

Un electrón relativista inmerso en un campo de materia (no relativista) será acelerado por el el campo coulombiano producido por los núcleos de los átomos que forman éste último. La materia puede estar constituída por núcleos desnudos (es decir, ionizada) o por átomos (núcleos apantallados por los electrones). En ambos casos, la interacción puede esquematizarse de la forma

$$
e+(e, N) \longrightarrow e+(e, N)+\gamma
$$

aunque la sección eficaz no es la misma. Esta viene dada por la expresión (Bosch-Ramon 2006)

$$
\frac{\mathrm{d} \sigma_{\mathrm{Brem}}\left(E_{e}, E_{\mathrm{ph}}\right)}{\mathrm{d} E_{\mathrm{ph}}}=\frac{4 \alpha_{\mathrm{ef}} r_{e}^{2} Z_{e}^{2}}{E_{\mathrm{ph}}} \phi\left(E_{e}, E_{\mathrm{ph}}\right),
$$

donde $\alpha_{\mathrm{ef}} \sim 1 / 137$ es la constante de estructura fina y $r_{e}=q_{e}^{2} / m_{e} c^{2}$ es el radio clásico del electrón. Para el caso de un núcleo desnudo, la función $\phi$ esta dada por

$$
\phi\left(E_{e}, E_{\mathrm{ph}}\right)=\left[1+\left(1-\frac{E_{\mathrm{ph}}}{E_{e}}\right)^{2}-\frac{2}{3}\left(1-\frac{E_{\mathrm{ph}}}{E_{e}}\right)\right]\left\{\ln \left[\frac{2 E_{e}\left(E_{e}-E_{\mathrm{ph}}\right)}{m_{e} c^{2} E_{\mathrm{ph}}}\right]-\frac{1}{2}\right\},
$$

mientras que para el caso en que el núcleo está completamente apantallado por todos los electrones

$$
\phi\left(E_{e}, E_{\mathrm{ph}}\right)=\left[1+\left(1-\frac{E_{\mathrm{ph}}}{E_{e}}\right)^{2}-\frac{2}{3}\left(1-\frac{E_{\mathrm{ph}}}{E_{e}}\right)\right] \ln \left(\frac{191}{Z_{e}^{1 / 3}}\right)+\frac{1}{9}\left(1-\frac{E_{\mathrm{ph}}}{E_{e}}\right) .
$$

El electrón entrega casi toda su energía a los fotones en estas interacciones, es decir, $E_{\mathrm{ph}} \sim E_{e}$, y por esto se dice que las pérdidas que sufre son catastróficas. Sin embargo, es posible hallar una expresión continua para el tiempo de enfriamiento. Para calcularlo, hacemos el mismo análisis que en el caso de las interacciones IC, sin embargo, debido a que hay dos parametrizaciones de $\sigma_{\mathrm{Brem}}$, para el caso en el que la materia está apantallada o aquel donde el medio está completamente ionizado, obtendremos dos expresiones diferentes 
para el tiempo de enfriamiento. Siendo $n_{\mathrm{m}}$ la densidad del campo de materia, en el primer caso tenemos

$$
t_{\mathrm{Brem}}^{\mathrm{neu}} \sim \frac{1.4 \times 10^{16}}{n_{\mathrm{m}} Z_{e}^{2}\left(\ln \left(\frac{183}{Z_{e}^{1 / 3}}\right)-\frac{1}{18}\right)} \mathrm{s},
$$

mientras que en el segundo

$$
t_{\mathrm{Brem}}^{\mathrm{ion}} \sim \frac{1.4 \times 10^{16}}{n_{\mathrm{m}} Z_{e}^{2}\left(\ln \left(\frac{E_{e}}{m_{e} c^{2}}\right)+0.36\right)} \mathrm{s} .
$$

Si ahora inyectamos una distribución $Q_{e}\left(E_{e}\right) \propto E_{e}^{-p}$ de electrones relativistas, las pérdidas por las interacciones con la materia casi no modifican $Q_{e}\left(E_{e}\right)$ ya que la dependencia con $E_{e}$ de $t_{\text {Brem }}$ es despreciable. Por esto,

$$
N_{e}\left(E_{e}\right) \sim Q_{e}\left(E_{e}\right) t_{\text {Brem }} \propto E_{e}^{-p} .
$$

La emisividad producida por una distribución de electrones $N_{e}\left(E_{e}\right)$ está dada por la ecuación (3.10), donde $n_{\mathrm{b}}$ es la densidad de materia y $\sigma=\sigma_{\text {Brem }}$, con lo cual hallamos

$$
J_{\mathrm{Brem}}\left(E_{\mathrm{ph}}\right) \sim \frac{c}{4 \pi} n_{\mathrm{m}} \int_{E_{\mathrm{ph}}}^{\infty} \frac{\mathrm{d} \sigma_{\mathrm{Brem}}\left(E_{e}, E_{\mathrm{ph}}\right)}{\mathrm{d} E_{\mathrm{ph}}} N_{e}\left(E_{e}\right) \mathrm{d} E_{e} .
$$

\subsubsection{Interacciones protón-protón}

Además de procesos asociados a cargas aceleradas, los fotones también pueden producirse por decaimientos de partículas. Por ejemplo, los piones neutros $\pi^{0}$ decaen en dos rayos gamma. Una manera de producir $\pi^{0}$ es a través de colisiones inelásticas protón-protón, en las cuales un protón relativista interactúa con un protón no relativista. El canal de interacciones $p p$ más importante para generar $\pi^{0}$ es el siguiente (Romero 2010):

$$
p+p \longrightarrow p+\Delta^{+}+\tilde{n}_{0} \pi^{0}+\tilde{n}_{ \pm}\left(\pi^{+}+\pi^{-}\right),
$$

donde $\pi^{+}$y $\pi^{-}$son piones cargados positiva y negativamente, respectivamente, y $\tilde{n}_{0}$ y $\tilde{n}_{ \pm}$ son las multiplicidades (números enteros positivos). En este proceso, el protón relativista pierde $\sim 50 \%$ de su energía, con lo cual la inelasticidad de la interacción es $f_{p p} \sim 0.5$. El decaimiento $\Delta^{+} \longrightarrow p+\pi^{0}$ entrega al $\pi^{0}$ aproximadamente un $17 \%$ de la energía $E_{p}$ del protón relativista. El $33 \%$ restante de $E_{p}$ es entregada al resto de los piones creados. Un $11 \%$ va a los piones neutros y un $22 \%$ a los cargados. Sin embargo, debido a que las multiplicidades $\tilde{n}_{0}$ y $\tilde{n}_{ \pm}$son grandes, la energía de cada una de estas partículas es poca. Es por esto que al $\pi^{0}$ con energía $\sim 0.17 E_{p}$ se lo llama "pión lider".

Para que la interacción $p p$ ocurra, la energía del protón relativista debe ser mayor que un valor umbral $E_{\mathrm{u}} \sim m_{p} c^{2}+2 m_{\pi} c^{2}\left(1+m_{\pi} / 4 m_{p}\right) \sim 1.22 \mathrm{GeV}$. La parametrización más reciente de la sección eficaz total de la interacción $p p$ es la dada por Kelner y colaboradores (2006):

$$
\sigma_{p p}\left(E_{p}\right)=\left(34.3+1.88 L+0.25 L^{2}\right)\left[1-\left(\frac{E_{\mathrm{u}}}{E_{p}}\right)^{4}\right]^{2} \mathrm{mb}
$$




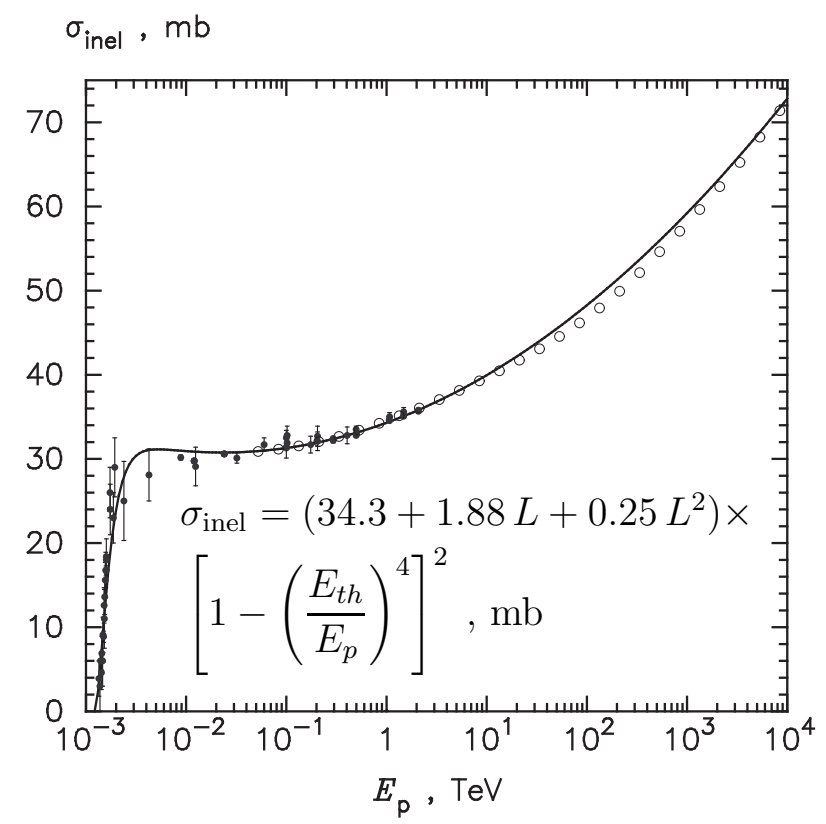

Figura 3.3: Sección eficaz de la interacción pp (Kelner et al. 2006).

donde $L=\ln \left(E_{p} / 1 \mathrm{TeV}\right)$ y su forma se muestra en la Figura 3.3

Como mostramos en la ecuación (3.7) el tiempo de enfriamiento depende de la sección eficaz de la interacción, con lo cual para diferentes parametrizaciones de ésta tendremos diferentes valores del tiempo de enfriamiento por $p p$. Sin embargo, debido a que todas las parametrizaciones halladas son parecidas y que la dependencia con $E_{p}$ es chica, usaremos una expresión sencilla pero útil para los fines de esta tesis. Considerando que $\sigma_{p p} \sim 30 \mathrm{mb}$ y que la inelasticidad de la interacción es $f_{p p} \sim 0.5$, el tiempo de enfriamiento para un medio de densidad $n_{\mathrm{m}}$ resulta

$$
t_{p p} \sim \frac{2 \times 10^{15}}{n_{\mathrm{m}}} \mathrm{s} .
$$

Como ocurre con el proceso Bremsstrahlung relativista, las pérdidas por $p p$ no modifican la forma del espectro de inyección. Si inyectamos protones relativistas con una distribución $Q_{p}\left(E_{p}\right) \propto E_{p}^{-p}$, al cabo de un tiempo $\gtrsim t_{p p}$ tendremos una distribución estacionaria de protones tal que

$$
N_{p}\left(E_{p}\right) \sim Q_{p}\left(E_{p}\right) t_{p p} \propto E_{p}^{-p} .
$$

Estos protones relativistas, al interactuar con el medio con densidad $n_{\mathrm{m}}$, producirán $\pi^{0}$ cuya emisividad está dada por:

$$
J_{\pi^{0}}\left(E_{\pi^{0}}\right)=\frac{c}{4 \pi} n_{\mathrm{m}} \int_{E_{p}^{\min }}^{E_{p}^{\max }} \frac{\mathrm{d} \sigma_{p p}\left(E_{\pi^{0}}, E_{p}\right)}{\mathrm{d} E_{\pi^{0}}} N_{p}\left(E_{p}\right) \mathrm{d} E_{p} .
$$

Para $E_{p} \lesssim 0.1 \mathrm{TeV}$, los piones creados tendrán una energía promedio $E_{\pi^{0}} \sim \kappa_{\pi} E_{p}$, donde $\kappa_{\pi} \sim 0.17$ (Gaisser 1990), y por lo tanto es posible considerar la aproximación $\delta\left(E_{\pi^{0}}-\right.$ 
$\left.\kappa_{\pi} E_{p}^{\text {cin }}\right)$ para calcular $J_{\pi^{0}}$. Siendo $E_{p}^{\text {cin }}=E_{p}-m_{p} c^{2}$ la energía cinética de los protones, se llega al siguiente resultado:

$$
J_{\pi^{0}}\left(E_{\pi^{0}}\right) \sim \frac{c}{4 \pi} \frac{\tilde{n}_{0}}{\kappa_{\pi}} n_{\mathrm{m}} N_{p}\left(m_{p} c^{2}+\frac{E_{\pi^{0}}}{\kappa_{\pi}}\right) \sigma_{p p}\left(m_{p} c^{2}+\frac{E_{\pi^{0}}}{\kappa_{\pi}}\right) .
$$

Sin embargo, la aproximación $\delta$ no es muy buena si $E_{p} \gtrsim 0.1 \mathrm{TeV}$ ya que en este caso la distribución de piones se ensancha en energía y la parte de más baja energía se hace más "blanda" para $E_{p}$ mayores, ya que se crean muchos piones de poca energía (es decir, la multiplicidad crece). En este rango de energía de los protones, las fórmulas adecuadas para calcular $J_{\pi^{0}}$ son las dadas por Kelner y colaboradores (2006). De esta manera, usando la aproximación $\delta$ (modificada) para $E_{p} \lesssim 0.1 \mathrm{TeV}$ y las parametrizaciones de Kelner para $E_{p} \gtrsim 0.1 \mathrm{TeV}$, la multiplicidad $\tilde{n}_{0}$ puede considerarse un parámetro libre y ajustarlo de tal manera que $J_{\pi^{0}}$ sea una función continua. Finalmente, calculada $J_{\pi^{0}}$, podemos obtener la emisividad de los rayos gamma a través de

$$
J_{\mathrm{ph}}\left(E_{\mathrm{ph}}\right)=2 \int_{E_{\pi^{0}}^{\min }}^{E_{\pi^{0}}^{\max }} \frac{J_{\pi^{0}}\left(E_{\pi^{0}}\right)}{\sqrt{E_{\pi^{0}}^{2}-m_{\pi}^{2} c^{4}}} \mathrm{~d} E_{\pi^{0}},
$$

donde $E_{\pi^{0}}^{\min }=E_{\mathrm{ph}}+m_{\pi}^{2} c^{4} /\left(4 E_{\mathrm{ph}}\right)$. El factor 2 en la expresión (3.41) tiene en cuenta que por cada $\pi^{0}$ se producen dos rayos gamma.

\section{Creación de pares electrón-positrón}

De acuerdo a (3.35), además de $\pi^{0}$ también se crean $\pi^{ \pm}$en las interacciones $p p$. Estos últimos luego decaen en muones que a su vez producen neutrinos y pares electrón-positrón $\left(e^{ \pm}\right)$. La mayoría de los pares producidos tienen energías $E_{e_{2}} \gg m_{e} c^{2}$ y por esto nos interesa conocer la distribución $N_{e_{2}}\left(E_{e_{2}}\right)$ de estas partículas ya que son una población adicional de leptones relativistas que se enfriarán de la misma manera que los electrones primarios acelerados, por ejemplo, en frentes de ondas de choque.

Si $N_{p}\left(E_{p}\right)$ es una ley de potencias, entonces la distribución de pares $e^{ \pm}$creados también será una ley de potencias $N_{e_{2}}\left(E_{e_{2}}\right)=K_{e_{2}}^{\prime} E_{e_{2}}^{-p_{2}^{\prime}}$, donde tanto $p_{2}^{\prime}$ como $K_{e_{2}}^{\prime}$ dependen de los parámetros de $N_{p}\left(E_{p}\right)$. Calculando $N_{e_{2}}\left(E_{e_{2}}\right)$ través de las fórmulas dadas por Kelner y colaboradores (2006) y considerando $K_{e_{2}}^{\prime}=1$ podemos hallar $p_{2}^{\prime}$ de hacer un ejuste gráfico del espectro calculado. Luego, conociendo el índice del espectro de los pares podemos hallar $K_{e_{2}}^{\prime}$ a través de la igualdad $u_{e_{2}}=f_{e^{ \pm}} u_{p}$, donde $f_{e^{ \pm}}$depende de $p_{2}$. Las cantidades $u_{e_{2}}$ y $u_{p}$ son las densidades de energía de los pares $e^{ \pm}$y de los protones relativistas, respectivamente.

\subsection{Absorción}

Anteriormente hemos visto diferentes procesos no térmicos que producen fotones. Sin embargo, éstos pueden a su vez ser absorbidos mediante interacciones con otras partículas. Estas interacciones pueden ocurrir con campos de materia, con campos magnéticos (Sturrock 1971) o con campos de fotones (Coppi \& Blandford 1990). 
Supongamos que un fotón de energía $E_{\mathrm{ph}}$ se propaga en un medio de densidad $n_{\mathrm{b}} \mathrm{y}$ contenido en una región de tamaño $L_{\mathrm{m}}$. Si la emisividad de los fotones es $J_{\mathrm{ph}}^{0}$, luego de atravesar la región de tamaño $L_{\mathrm{m}}$, la emisividad será reducida a $J_{\mathrm{ph}}$ de manera tal que

$$
J_{\mathrm{ph}}\left(E_{\mathrm{ph}}\right)=J_{\mathrm{ph}}^{0}\left(E_{\mathrm{ph}}\right) e^{-\tau} .
$$

La profundidad óptica del medio, $\tau$, se define de la siguiente manera:

$$
\tau\left(E_{\mathrm{ph}}\right)=\int_{0}^{L_{\mathrm{m}}} \int_{E_{\mathrm{b}}^{\min }}^{\infty} n_{\mathrm{b}}\left(E_{\mathrm{b}}, r\right) \sigma_{\mathrm{abs}}\left(E_{\mathrm{ph}}, E_{\mathrm{b}}\right) \mathrm{d} E_{\mathrm{b}} \mathrm{d} r
$$

donde $\sigma_{\text {abs }}$ es la sección eficaz del proceso y $E_{\mathrm{b}}^{\mathrm{min}}$ es la energía mínima de las partículas del medio para que ocurra la interacción entre una de ellas y un fotón. Si $\tau \ll 1$ entonces no hay absorción y el medio se dice ópticamente delgado. Por otro lado, si $\tau \geq 1$ la absorción puede ser significativa y el medio se dice ópticamente grueso.

\subsubsection{Absorción por creación de pares electrón-positrón}

En esta tesis sólo consideraremos un mecanismo de absorción; aquel en el cual dos fotones se aniquilan produciendo un par $e^{ \pm}$de la siguiente manera

$$
\gamma+\gamma \longrightarrow e^{+}+e^{-}
$$

Si consideramos que el fotón de energía $E_{\mathrm{ph}}$ producido en la fuente se propaga en un gas isotrópico y (quasi)monoenergético de fotones de energía $E_{\mathrm{ph}}^{0}$, para que la interacción ocurra, debido a que se crean dos partículas con masa $m_{e}$, la energía umbral de los fotones

debe ser tal que $E_{\mathrm{ph}} E_{\mathrm{ph}}^{0} \geq 2 m_{e}^{2} c^{4}$. La sección eficaz de esta interacción fotón-fotón es (Coppi \& Blandford 1990)

$$
\sigma_{\gamma \gamma}\left(E_{\mathrm{ph}}, E_{0}\right) \sim \sigma_{\mathrm{T}} \frac{\left(x_{\gamma \gamma}-1\right)^{1 / 3}}{x_{\gamma \gamma}^{5 / 2}}\left(\frac{1}{2 x_{\gamma \gamma}^{1 / 2}}+\frac{3}{4} \ln \left(x_{\gamma \gamma}\right)\right) H\left(x_{\gamma \gamma}-1\right)
$$

donde $x_{\gamma \gamma} \equiv E_{\mathrm{ph}} E_{\mathrm{ph}}^{0} / m_{e}^{2} c^{4}$ y $H\left(x_{\gamma \gamma}-1\right)$ es la función de Heaviside. Como se muestra en la Figura [3.4 $\sigma_{\gamma \gamma}$ tiene un máximo cuando $E_{\mathrm{ph}} E_{\mathrm{ph}}^{0} \sim 3.7 m_{e}^{2} c^{4}$.

Si la densidad del gas de fotones en el cual se propaga el rayo gamma es $n_{\mathrm{phm}}$, la profundidad óptica será:

$$
\tau_{\gamma \gamma}\left(E_{\mathrm{ph}}, E_{\mathrm{ph}}^{0}\right)=n_{\mathrm{ph}} \sigma_{\gamma \gamma}\left(E_{\mathrm{phm}}, E_{\mathrm{ph}}^{0}\right) L_{\mathrm{m}} .
$$




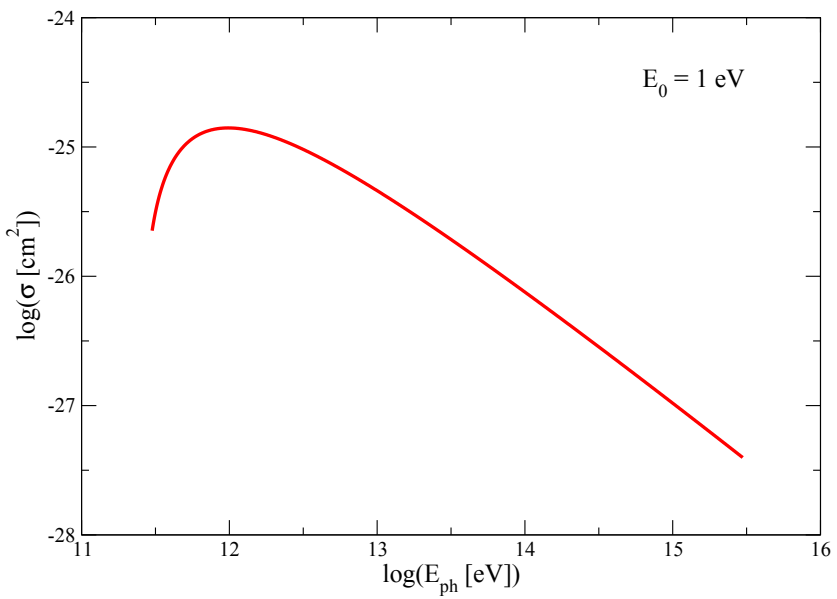

Figura 3.4: Sección eficaz isotrópica $\left(\sigma_{\gamma \gamma}\right)$ del proceso de creación de pares $e^{ \pm}$por aniquilación de fotones. El grafico corresponde a $E_{\mathrm{ph}}^{0}=1 \mathrm{eV}$. 


\section{Parte II}

\section{Estudios de fuentes a diferentes escalas}





\section{Capítulo 4}

\section{Objetos estelares jóvenes}

\subsection{Introducción}

Las estrellas se clasifican de acuerdo a su masa $M_{\star}$ en de gran masa $\left(M_{\star} \geq 8 M_{\odot}\right)$ y de baja masa $\left(M_{\star} \leq 8 M_{\odot}\right)$. Numerosas de éstas últimas están cerca y son fáciles de detectar. Por esto se conoce bastante de su formación y evolución. Sin embargo, no ocurre lo mismo con las estrellas de gran masa, las cuales se encuentran embebidas en grandes condensaciones de gas y polvo con lo cual la extinción de la luz que emiten es significativa y poco llega de ella a nuestros detectores. Si además tenemos en cuenta que el tiempo de vida de estas estrellas es muy corto $(\sim 1-10 \mathrm{Myr})$, la observación y detección de las estrellas tempranas (tipos espectrales $\mathrm{O}$ y B) en cada estado evolutivo es extremadamente difícil. Es por esto que el estudio de la formación de las estrellas de gran masa es uno de los grandes tópicos de la astrofísica actual.

La formación estelar comienza cuando una nube de gas en el espacio se vuelve inestable y colapsa bajo la acción de su propia gravedad. Durante el colapso, la nube se fragmenta. Esto es todo lo que puede decirse respecto de la etapa inicial de la formación de una estrella, ya que se sabe muy poco de como ocurre la fragmentación de la nube. Luego, la nube queda dividida en varias partes, cada una de ellas con una distribución de densidad inhomogénea la cual induce el proceso de acreción y así se forman núcleos más densos. Dependiendo de la masa de estos núcleos y de la tasa de acreción de materia, se formarán estrellas de diferentes masas.

Para los núcleos poco densos que luego darán lugar a las estrellas de baja masa, la secuencia de eventos hasta llegar a la formación de la estrella pareciera ser clara y la resumimos de la siguiente manera (Shu et al. 1987):

1. Un estado inicial en el cual la región central de los núcleos densos se contrae, intensificando así su campo gravitacional y formando una protoestrella en cada núcleo.

2. Un estado de acreción caracterizado por la formación de un disco alrededor de cada protoestrella y a través del cual la misma acreta materia del medio circundante.

3. La fase en la cual se producen los flujos bipolares (outflows o jets dependiendo del 


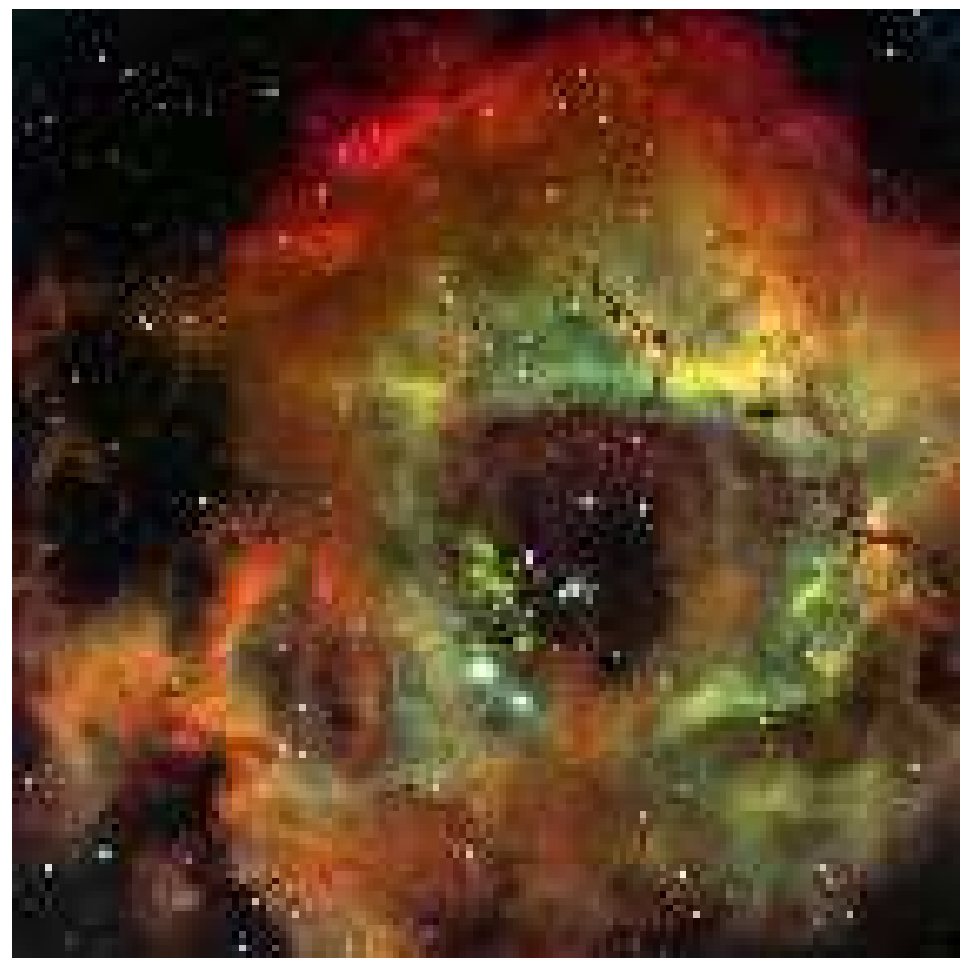

Figura 4.1: Región de formación estelar masiva conocida como Messier 20. Esta imagen ha sido tomada en el IR por el satélite Spitzer.

ángulo de colimación de los mismos), por los cuales la protoestrella deposita materia con momento angular y energía cinética en sus alrededores. Estos flujos bipolares, al chocar violentamente con el medio circundante, producen los llamados objetos Herbig-Haro (HH).

4. Finalmente, la etapa en la cual la protoestrella se sitúa en la secuencia principal de edad cero (ZAMS, por Zero Age Main Sequence). Es en este momento cuando comienza la combustión eficiente de hidrógeno en el núcleo de la estrella.

Por otro lado, el mecanismo de formación de las estrellas de gran masa no es tan claro hoy en día. Si bien no hay dudas de que las estrellas tempranas se formarían en nubes moleculares gigantes, el proceso de fragmentación de dicha nube para formar grumos autogravitantes de gran masa es aún desconocido, como así también los subsecuentes procesos dinámicos que darían origen a estas estrellas. Se han planteado dos modelos para explicar la formación de las estrellas de gran masa: uno es aquel en el cual el mecanismo de formación es similar al que opera en las estrellas de baja masa (Shu et al. 1987), mientras que el otro es un modelo tipo jerárquico donde la coalescencia de estrellas menos masivas daría origen a estrellas con más masa (Bonnell et al. 1998). Observaciones recientes de regiones de formación estelar gigantes y masivas (ver la Figura 4.1) han detectado la presencia de 
discos de acreción y de flujos bipolares emanando de protoestrellas de gran masa, como describiremos a continuación.

Cúmulos: Observaciones en radio de regiones de formación estelar masivas de la Galaxia y de galaxias cercanas muestran que las estrellas de gran masa se forman en grupos. Las fuentes IRAS1 más luminosas asociadas a regiones compactas de gas ionizado (y por ende a objetos estelares jóvenes), muestran una morfología compleja cuando se las observa en frecuencias radio. Garay y colaboradores (1993) sugieren que la presencia de estrellas embebidas en la región (un cúmulo de estrellas O y B) excitarían el gas de la misma y esto produciría la compleja estructura observada. Algunas de las regiones más estudiadas, que contienen estrellas masivas, son W3 Orion-Trapezium y NGC 2024. La densidad típica de estos cúmulos de estrellas jóvenes es de $\sim 10^{4}$ estrellas por $\mathrm{pc}^{-3}$ y el tama no es de $\sim 0.2-0.4 \mathrm{pc}$.

Discos de acreción: Los discos detectados en regiones de formación de estrellas de gran masa tienen un diámetro entre 0.1 y 1 pc, y sus masas pueden ir desde las 10 a las $2000 \mathrm{M}_{\odot}$. Una de las evidencias observacionales más importantes de la existencia de discos circumestelares en regiones de formación estelar masiva es la detección de la emisión de gas y polvo en el infrarrojo (IR).

Jets y outflows: La presencia de flujos bipolares en la formación de estrellas de gran masa fue determinada recientemente mediante observaciones en radio. Sobre una muestra de aproximadamente 120 regiones de formación de estrellas tempranas, en el $90 \%$ de los casos se observó gas moviendosé a velocidades altas (> $\left.100 \mathrm{~km} \mathrm{~s}^{-1}\right)$. Luego, si las velocidades elevadas del gas están asociadas a la presencia de outflows, puede concluirse que éstos son comunes también en la formación de las estrellas de gran masa. Algunas de las propiedades de los outflows detectados en regiones de formación de estrellas tempranas son las siguientes:

- Tienen una masa promedio de $130 \mathrm{M}_{\odot}$, aunque en algunos caso puede alcanzar valores de hasta $\sim 4800 \mathrm{M}_{\odot}$.

- La tasa de pérdida de masa abarca desde $\sim 3 \times 10^{-5} \mathrm{M}_{\odot} \mathrm{yr}^{-1}$ hasta $\sim 3 \times 10^{-2} \mathrm{M}_{\odot}$ $\mathrm{yr}^{-1}$.

- La energía cinética toma valores desde $\sim 10^{46}$ hasta $\sim 6 \times 10^{48} \mathrm{erg}$.

Estas cantidades son $\sim 100$ veces mayores que las correspondientes a los outflows presentes durante la formación de estrellas de baja masa. Luego puede concluirse que los outflows de alta masa inyectan mayor cantidad de energía al medio circundante que los de baja masa. En la Figura 4.2 se muestra el outflow detectado en la fuente AFGL 2591.

Los resultados observacionales descriptos anteriormente sugieren que las estrellas de gran masa se forman a través de un mecanismo similar al que opera para formar las estrellas de baja masa, pero con una tasa de acreción de materia a través de los discos y de eyección a través de los outflows $\sim 100$ veces mayor a la observada en estrellas de baja masa. Sin embargo, no se han detectado discos de acreción en estrellas de $M_{\star}>50 M_{\odot}$,

\footnotetext{
${ }^{1}$ Se conocen como fuentes IRAS a aquellas detectadas por el satélite IR que lleva dicho nombre.
} 


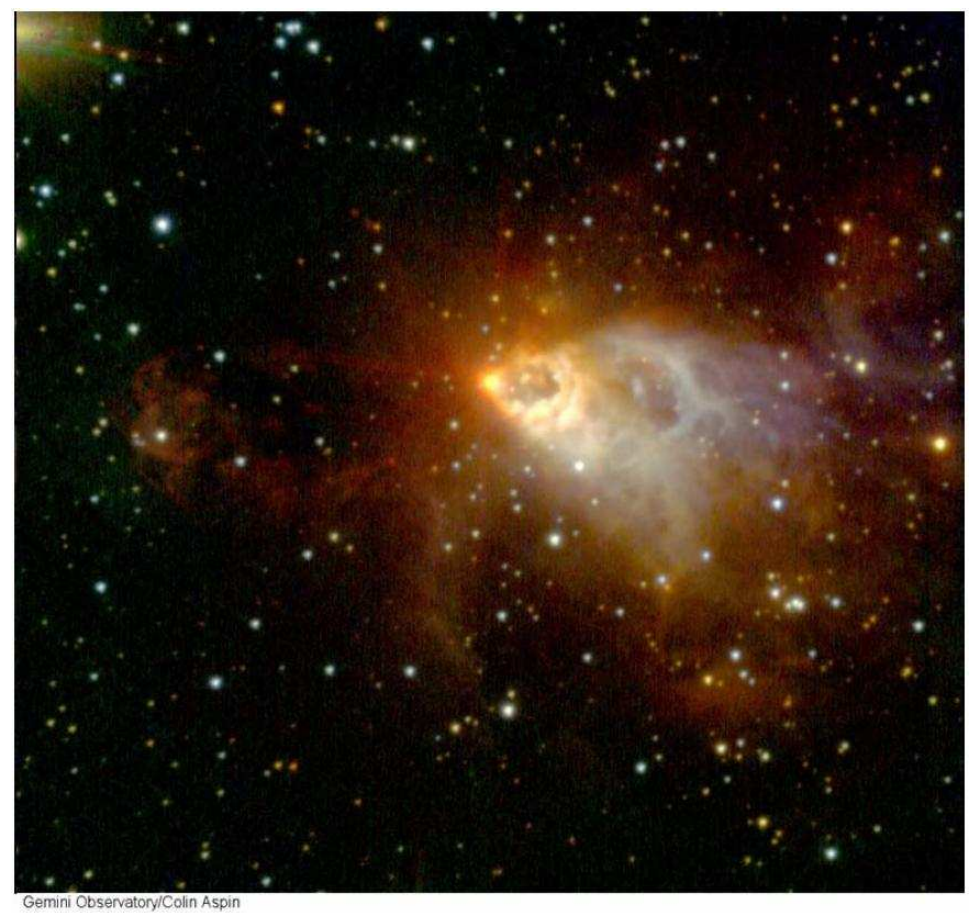

Figura 4.2: Outflow masivo asociado a la fuente AFGL 2591. La imagen ha sido tomada en el IR por el telescopio Gemini Norte (Zinnecker \& Yorke, 2007). 
las cuales al ser tan luminosas $\left(L_{\star}>10^{5} L_{\odot}\right)$ podrían evaporar los discos a través de un mecanismo conocido como "fotoerosión". Zinnecker \& Yorke (2007) sugieren que el mecanismo para formar a las estrellas más masivas $\left(M_{\star}>50 M_{\odot}\right)$ no sería un simple escaleo del proceso de formación de las de baja masa. Los fuertes vientos de las estrellas más masivas (junto a la fuerte radiación) disiparían el disco y favorecerían las interacciones entre protoestrellas cercanas, siendo entonces la coalescencia de estrellas o protoestrellas el mecanismo de formación.

Por todo lo expuesto anteriormente, es claro que todavía hay mucho para decir respecto de como se forman las estrellas de gran masa. Todos los estudios y observaciones de regiones de formación estelar masivas son en general en las bandas de radio, IR y rayos X, mientras que a más altas energías sólo se han observado algunos cúmulos y asociaciones de estrellas O y B. Hasta el momento, no se han detectado estrellas en formación en el rango de los rayos gamma, aunque posiblemente se han detectado rayos gamma de estrellas de gran masa evolucionadas, en general Wolf-Rayet (WR) o estrellas luminosas variables azules (LBV, por Luminous Blue Variable). Las regiones en las que se ha detectado emisión significativa en rayos gamma son Westerlund 2 (Aharonian et al. 2007), Cygnus OB2 y Carina (Tavani et al. 2009a). Las partículas relativistas que emiten esta radiación gamma detectada podrían acelerarse en las ondas de choque producidas por las explosiones de supernovas dentro de la región o bien en choques de vientos de estrellas de gran masa. Sin embargo, estos cúmulos o asociaciones de estrellas tempranas también pueden ser regiones de formación estelar, aunque nada se había dicho de la posible emisión a altas energías de las protoestrellas masivas. Los rayos gamma, de ser producidos en regiones de formación estelar, aportarían información adicional a la que se obtiene de otras frecuencias sobre como es el mecanismo de formación de las estrellas de gran masa, motivando esto el primer estudio realizado en esta tesis.

Sabemos que para emitir rayos gamma es necesaria la presencia de partículas no térmicas. Una de las evidencias de la existencia de electrones relativistas es la detección en frecuencias radio de emisión no térmica. Se han detectado en radio muchos YSOs de gran masa, pero sólo algunos con emisión no térmica. Para nuestro estudio hemos elegido una fuente muy particular detectada en radio (no térmico) por Garay y colaboradores (2003). Estos autores han detectado en frecuencias radio un sistema triple compuesto por una protoestrella de gran masa y dos lóbulos. Este sistema se asocia con la fuente infrarroja IRAS $16547-4247$.

\subsection{La fuente IRAS 16547-4247}

La fuente IRAS 16547-4247 es una región de formación estelar de gran masa ubicada a una distancia $d_{\mathrm{nm}} \sim 2.9 \mathrm{kpc}$ y tiene un diámetro angular de $\sim 27^{\prime \prime}$ (Garay et al. 2003), que a la distancia $d_{\mathrm{nm}}$ corresponde a un tamaño lineal $D_{\mathrm{nm}} \sim 0.38$ pc $\left(\sim 1.1 \times 10^{18} \mathrm{~cm}\right)$. La luminosidad bolométrica es $L_{\star} \sim 6.2 \times 10^{4} L_{\odot} \sim 2.4 \times 10^{38} \mathrm{erg} \mathrm{s}^{-1}$ donde $L_{\odot}=$ $4 \times 10^{33} \mathrm{erg} \mathrm{s}^{-1}$ es la luminosidad bolométrica del Sol. De la densidad columnar de hidrógeno estimada, resulta que la masa de la nube es $M_{\mathrm{nm}} \sim 9 \times 10^{2} M_{\odot} \sim 10^{36} \mathrm{gr}$, siendo $M_{\odot} \sim$ 
$2 \times 10^{33}$ gr una masa solar, y así la densidad numérica promedio de partículas resulta $n_{\mathrm{nm}} \sim 5.2 \times 10^{5} \mathrm{~cm}^{-3}$. Los parámetros conocidos y estimados de esta fuente están listados en la Tabla 4.1.

\begin{tabular}{ll}
\hline \hline Parámetro & Valor \\
\hline Distancia & $d_{\mathrm{nm}}=2.9 \mathrm{kpc}$ \\
Tamaño & $D_{\mathrm{nm}}=0.38 \mathrm{pc}$ \\
Masa & $M_{\mathrm{nm}}=9 \times 10^{2} M_{\odot}$ \\
Densidad & $n_{\mathrm{nm}}=5.2 \times 10^{5} \mathrm{~cm}^{-3}$ \\
Luminosidad & $L_{\star}=6.2 \times 10^{4} L_{\odot}$ \\
\hline
\end{tabular}

Tabla 4.1: Parámetros de la fuente IRAS 16547-4247.

Mediante observaciones llevadas a cabo con el interferómetro ATCA (Australia Telescope Compact Array), Garay y colaboradores (2003) detectaron un sistema triple embebido en la nube molecular asociada a la fuente IRAS 16547-4247. Este sistema está formado por una protoestrella de gran masa y dos lóbulos alineados con ésta y ubicados simétricamente a una distancia $\sim 0.14 \mathrm{pc}\left(\sim 10^{\prime \prime}\right)$ de la protoestrella central, como se muestra en la Figura 4.3. Con observaciones posteriores realizadas con el radiotelescopio VLA (Very Large Array) por Rodríguez y colaboradores (2005) a las frecuencias $\nu=8.46$ y $14.9 \mathrm{GHz}$, se calculó el índice espectral $\alpha$ del flujo observado $S_{\nu}\left(\propto \nu^{-\alpha}\right)$ de cada componente del sistema triple. Para la fuente central se estimó $\alpha=-0.33 \pm 0.05$ mientras que para los lóbulos norte y sur, $\alpha=0.17 \pm 0.39$ y $0.59 \pm 0.15$, respectivamente. Estos valores son muy similares a los calculados por Garay y colaboradores (2003) con los datos de ATCA.

Si la emisión continua detectada en radio es producida únicamente a través de interacciones libre-libre, entonces el espectro observado debe corresponder a un valor $\alpha<0.1$, independientemente de las características del emisor. Por otro lado, valores $\alpha \gg 0.1$ son debidos a radiación sincrotrón ópticamente delgada (Rodríguez et al. 1993). Con este criterio, la emisión radio de la fuente central sería térmica, asociada a la radiación libre-libre de la base del jet y que se conoce como jet térmico, mientras que la emisión proveniente de los lóbulos, en particular del sur, sería no térmica. Sin embargo, para confirmar que la emisión de los lóbulos es no térmica, deben hacerse estudios polarimétricos.

La detección de emisión no térmica proveniente de los lóbulos es una evidencia de la presencia de electrones relativistas en tal lugar (donde esta radiación es producida). Estas partículas serían aceleradas hasta energías relativistas en los choques producidos cuando el jet es frenado por el medio en el cual se está propagando, es decir, la nube molecular. En esta tesis modelamos a la fuente como se muestra en la Figura 4.4.

Además de radiación sincrotrón, los electrones relativistas podrían producir fotones a más altas energías por otros mecanismos radiativos como el Bremsstrahlung relativista y la dispersión Compton inversa. Por otro lado, debido a que el mecanismo de Fermi puede acelerar también protones, las interacciones $p p$ entre protones acelerados y protones fríos de la nube son también posibles. Como consecuencia de esto se producen, además de los fotones, pares $e^{ \pm}$secundarios los cuales se enfrían de la misma manera que los electrones primarios. 

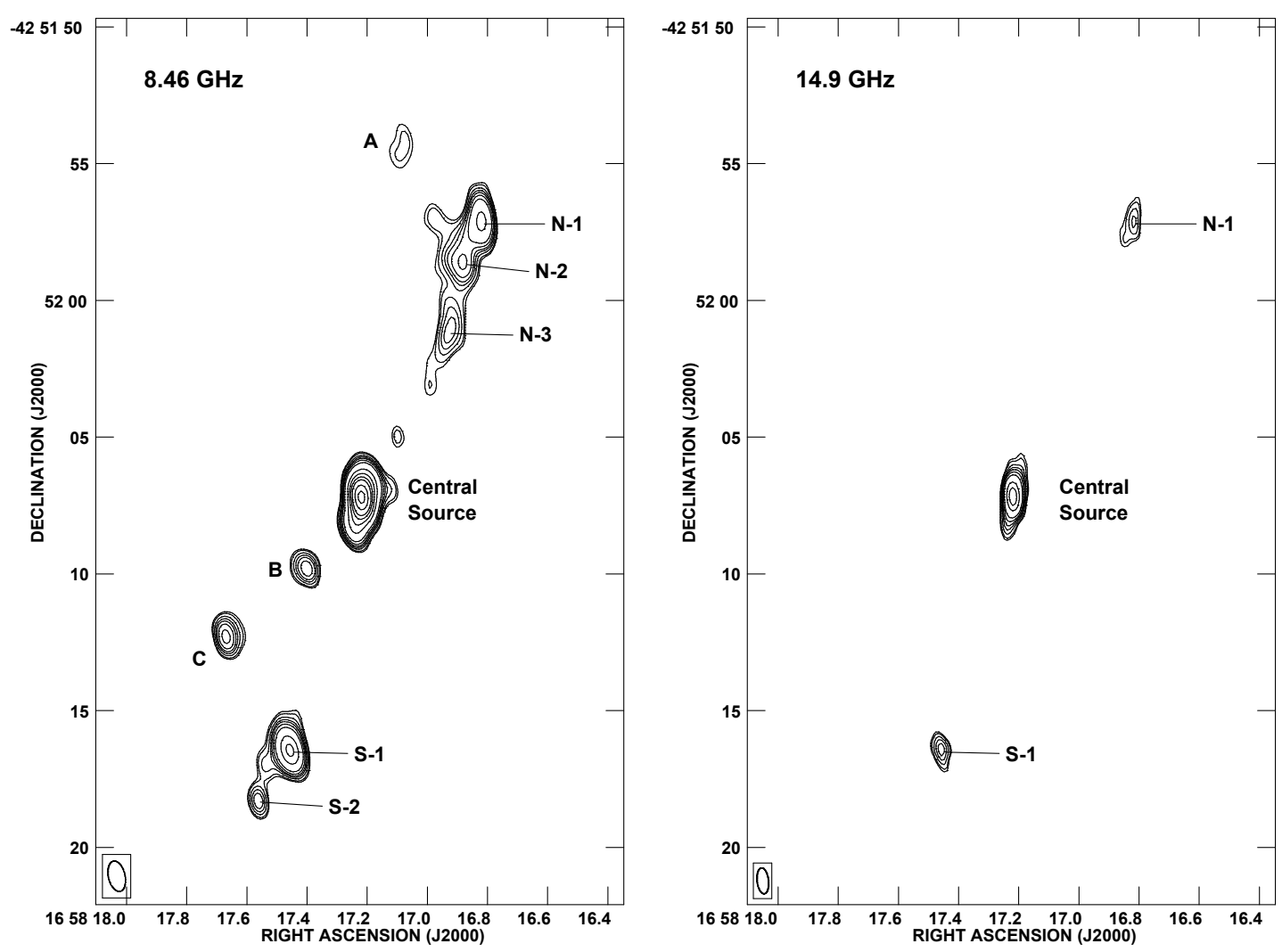

Figura 4.3: Imágenes tomadas con VLA de la fuente IRAS 16547-4247 en $\nu=8.46$ (izquierda) y $14.9 \mathrm{GHz}$ (derecha). A éstas frecuencias se ve claramente el sistema triple compuesto por la fuente central (central source) y los lóbulos norte (N) y sur (S) (Rodríguez et al. 2005).

De esta menera, podríamos tener tres poblaciones diferentes de partículas no térmicas: electrones y protones primarios y pares secundarios $e^{ \pm}$. A continuación estudiamos como se producen estas poblaciones de partículas relativistas.

\subsection{Población de partículas relativistas}

La emisión detectada en frecuencias radio proveniente de los lóbulos de la fuente IRAS 16547-4247 es no térmica (de acuerdo al criterio determinado por Rodríguez et al. 1993) y consideramos que dicha radiación es producida por electrones relativistas acelerados en los choques teminales de los jets del YSO o por pares $e^{ \pm}$producidos por protones relativistas acelerados en los mismos choques. Mientras los jets se propagan (libremente) por la nube molecular van chocando el material de ésta produciendo así un bow shock en el medio. Cuando los jets son frenados significativamente por este medio externo chocado, se forma un choque reverso en los jets, como se comentó en la sección 2.5.2. A continua- 


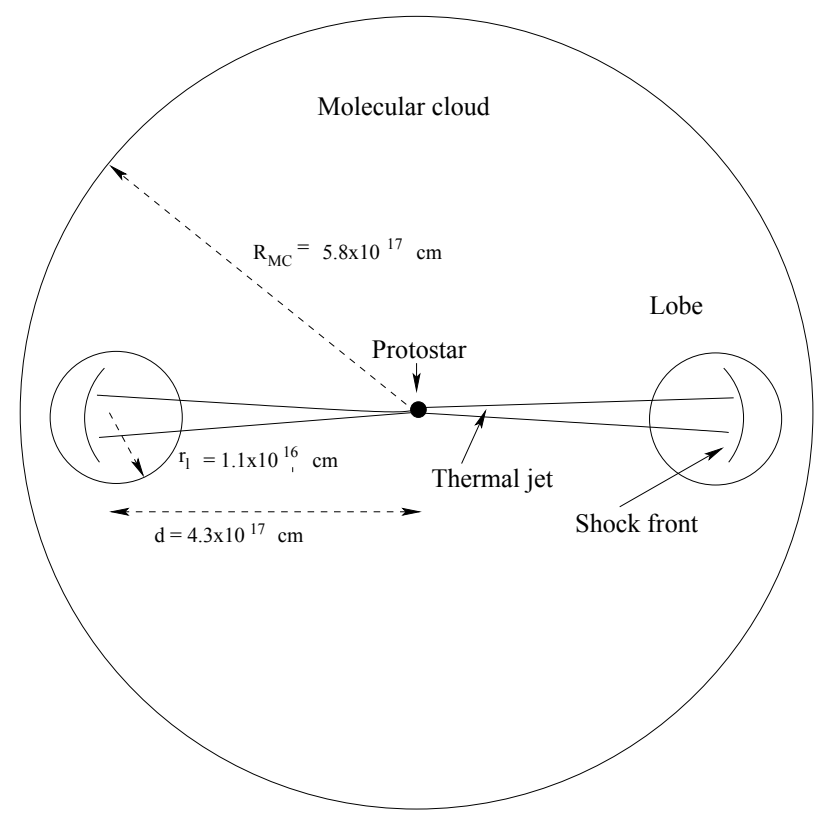

Figura 4.4: Esquema del modelo propuesto para la fuente IRAS 16547-4247 (Araudo et al. 2007).

ción haremos una descripción más detellada que la expuesta en el Capítulo 2, estudiando específicamente el caso de los YSOs, para luego estimar la población de partículas relativistas en los lóbulos de la fuente IRAS 16547-4247. En particular, nos concentramos en el estudio del lóbulo sur, ya que la emisión radio proveniente de éste tiene un indice espectral puramente no térmico, $\alpha \sim 0.6$, mientras que la emisión del lóbulo norte podría estar contaminada de radiación térmica, ya que el $\alpha(\sim 0.2)$ es más cercano al valor crítico $\alpha_{\mathrm{c}}=0.1$.

\subsubsection{Choques terminales}

Para determinar las características de los choques terminales, un parámetro importante es el contraste entre la densidad del jet y de la nube, $\chi_{\text {yso }} \equiv n_{\mathrm{j}} / n_{\mathrm{nm}}$. Además de $\chi_{\text {yso }}$ es necesario conocer la velocidad del $j e t, v_{\mathrm{j}}$, para poder estimar las velocidades del bow shock $\left(v_{\text {bs }}\right)$ y del choque reverso $\left(v_{\mathrm{r}}\right)$, de la siguiente manera (Bosch-Ramon et al. 2010):

$$
v_{\mathrm{bs}} \sim \frac{v_{\mathrm{j}}}{\left(1+\chi_{\mathrm{yso}}^{-1 / 2}\right)} \quad \text { y } \quad v_{\mathrm{r}} \sim v_{\mathrm{j}}-\frac{3}{4} v_{\mathrm{bs}},
$$

donde se ha supuesto que el bow shock es adiabático. Debido a que la velocidad de los jets de la fuente IRAS 16547-4247 no se conoce, adoptamos para esta magnitud un valor que sea concordante con aquellos medidos en otros YSOs de gran masa. Martí y colaboradores (1995) han determinado para el jet térmico de la fuente HH 80-81 una velocidad que ronda 
los 600 y $1400 \mathrm{~km} \mathrm{~s}^{-1}$. Luego, para la fuente que estamos estudiando en este capítulo consideramos $v_{\mathrm{j}}=1000 \mathrm{~km} \mathrm{~s}^{-1}$.

Si bien no conocemos el valor de $\chi_{\mathrm{yso}}$, porque no conocemos $n_{\mathrm{j}}$ en la localización de los lóbulos $\left(Z_{\mathrm{j}} \sim 4 \times 10^{17} \mathrm{~cm}\right)$, podemos determinar $v_{\mathrm{bs}}$ a través del tiempo de vida del jet, $\tau_{\mathrm{j}}$. Esta escala temporal se define como aquella en la cual el jet recorre la distancia $Z_{\mathrm{j}}$ a la que se detectan los lóbulos. Esto es, $\tau_{\mathrm{j}} \sim Z_{\mathrm{j}} / v_{\mathrm{bs}}$ y en el caso de la fuente IRAS 165474247, $\tau_{\mathrm{j}} \sim 10^{11} \mathrm{~s}$ (Garay et al. 2007) con lo cual, si $Z_{\mathrm{j}} \sim 4.3 \times 10^{17} \mathrm{~cm}$ obtenemos $v_{\mathrm{bs}} \sim 5 \times 10^{6} \mathrm{~cm} \mathrm{~s}^{-1}$. Con este valor de $v_{\mathrm{bs}} \mathrm{y}$ las ecuaciones (4.1) hallamos $\chi_{\mathrm{yso}} \sim 2.5 \times 10^{-3}$ y luego $n_{\mathrm{j}}\left(Z_{\mathrm{j}}\right) \sim 1.25 \times 10^{3} \mathrm{~cm}^{-3}$. Siendo $v_{\text {bs }} \ll v_{\mathrm{j}}$, resulta que $v_{\mathrm{r}} \sim v_{\mathrm{j}}$.

La región entre ambos choques puede tener un estructura muy compleja ya que estos choques pueden ser radiativos y en la superficie de discontinuidad entre ambos medios chocados (conocida como "superficie de trabajo") se pueden desarrollar inestabilidades de RT que mezclan material de ambos medios (Blondin et al. 1989). Para estimar si los choques son radiativos o no, comparamos el tiempo de enfriamiento por emisión térmica, $t_{\text {ter }}$, con el tiempo característico $t_{\text {carac }}$ en el cual el medio chocado recorre una distancia $\sim R_{\text {lob. }}$. Este último resulta $t_{\text {carac }} \sim 4 R_{\mathrm{lob}} / v_{\mathrm{ch}}$. Para estimar $t_{\text {ter }}$ consideramos la ecuación (2.55) $\mathrm{y}$ obtenemos (Bosch-Ramon et al. 2010):

$$
t_{\mathrm{rad}} \sim 2 \times 10^{-25} \frac{v_{\mathrm{ch}}^{2}}{n_{0} \Lambda(T)} \sim 7 \times 10^{-18} \frac{v_{\mathrm{ch}}^{3.2}}{n_{0}} \mathrm{~s},
$$

donde hemos considerado la función de enfriamiento $\Lambda(T)=7 \times 10^{-19} T^{-0.6}$ (Bosch-Ramon et al. 2010) y las condiciones de salto (2.27) y (2.28). Luego, comparando $t_{\text {rad }}$ con $t_{\text {carac }}$ tenemos que los choques son radiativos si $t_{\text {rad }}<t_{\text {carac }}$, es decir, si

$$
v_{\mathrm{ch}}<\left(\frac{4 R_{\mathrm{lob}} n_{0}}{7 \times 10^{-18}}\right)^{1 / 4.2} \sim 1.6 \times 10^{8} n_{0}^{1 / 4.2} \mathrm{~cm} \mathrm{~s}^{-1} .
$$

Considerando $v_{\mathrm{ch}}=v_{\mathrm{bs}} \mathrm{y} n_{0}=n_{\mathrm{nm}}$ para el bow shock y $v_{\mathrm{ch}}=v_{\mathrm{r}} \mathrm{y} n_{0}=n_{\mathrm{j}}\left(Z_{\mathrm{j}}\right)$ para el choque reverso, obtenemos que mientras que el primero resulta radiativo, el último es adiabático. Luego la densidad del material chocado de la nube aumenta más de un factor 4, pudiendo llegar hasta valores mucho más grandes que el valor adiabático. Con esto, si el factor de mezcla de ambos medios chocados es grande, podemos considerar que las partículas aceleradas en el choque reverso pueden interactuar, además de con el material del jet chocado, con material chocado de la nube, cuya densidad es mucho más alta y así las pérdidas por Bremsstrahlung relativista e interacciones $p p$ mucho más eficientes.

En resumen, tenemos que los lóbulos son regiones en donde hay choques fuertes que pueden acelerar partículas hasta energías relativistas. Estas partículas, además de producir la radiación sincrotrón observada, pueden también radiar por otros mecanismos no térmicos, como veremos a continuación.

\subsubsection{Aceleración de partículas y pérdidas radiativas}

Como vimos en el Capítulo 2, la eficiencia para acelerar partículas mediante el mecanismo de Fermi de tipo I es $\propto v_{\mathrm{ch}}^{2}$. Por esto, nos concentramos en la aceleración de partículas 
en el choque reverso, ya que $v_{\mathrm{r}} \gg v_{\mathrm{bs}}$.

El tiempo de aceleración depende, además de $v_{\mathrm{r}}^{2}$, del campo magnético en la región de aceleración, que coincide con el lóbulo sur, y denotaremos $B_{\text {lob. Para estimar } B_{\text {lob }}}$ consideramos que la densidad de energía magnética, $B_{\text {lob }}^{2} /(8 \pi)$, es igual a la densidad de energía $u_{n t}$ de las partículas no térmicas. Sabemos que hay leptones relativistas en el lóbulo que producen la emisión sincrotrón detectada en frecuencias radio. Estos leptones pueden ser electrones primarios $\left(e_{1}\right)$ acelerados en el choque reverso o bien pares $e^{ \pm}\left(e_{2}\right)$ producidos por interacciones inelásticas $p p$ de protones $(p)$ acelerados en el mismo choque con el material de la nube molecular. El caso más general que podemos plantear es

$$
\frac{B_{\mathrm{lob}}^{2}}{8 \pi}=u_{\mathrm{nt}}=u_{e_{1}}+u_{p}+u_{e_{2}}
$$

donde $u_{e_{1}}$ y $u_{p}$ son las densidades de energía de los electrones y protones primarios, respectivamente, y $u_{e_{2}}$ corresponde a los pares $e^{ \pm}$. En cada caso $\left(i=e_{1}, p, e_{2}\right)$ :

$$
u_{i}=\int_{E_{i}^{\min }}^{E_{i}^{\max }} E_{i} n\left(E_{i}\right) \mathrm{d} E_{i}
$$

siendo $n\left(E_{i}\right)=k_{i}^{\prime} E_{i}^{-p_{i}^{\prime}} \exp \left(-E_{i} / E_{i}^{\max }\right)$ la distribución de energía de las partículas $i$ por unidad de volumen, es decir, $\left[n\left(E_{i}\right)\right]=\mathrm{cm}^{-3} \operatorname{erg}^{-1}$ y $E_{i}^{\min }$ y $E_{i}^{\text {max }}$ las energías mínima y máxima de la distribución $n\left(E_{i}\right)$. Como no tenemos ningún indicio observacional de la presencia de protones relativistas en el lóbulo sur, la única manera de estimar $u_{p}$ es a través de un parámetro fenomenológico $a$, imponiendo que $u_{p}=a u_{e_{1}}$. En esta tesis consideramos 3 casos: $a=0,1$ y 100. El valor $a=100$ se toma porque el espectro de los rayos cósmicos galácticos pareciera indicar que la fracción de protones a electrones es $\sim 100$. Por otro lado $u_{e_{2}}=f_{e^{ \pm}} u_{p}$, donde $f_{e^{ \pm}}<1$ (Kelner et al. 2006).

El espectro observado en radio del lóbulo sur puede ajustarse con una ley de potencias $\left(\propto \nu^{-\alpha}\right)$ con índice $\alpha=0.59$. Luego, el índice espectral de los leptones relativistas que producen esta radiación sincrotrón resulta $p_{e}^{\prime}=2 \alpha+1=2.18$. En los casos $a=1$ y $a=$ 100 consideramos además una distribución $n_{p}\left(E_{p}\right)$ de protones relativistas cuya población de pares $e^{ \pm}$producidos en las interacciones $p p$ puede estimarse como se explicó en el Capítulo 3 En el caso con $a=1, u_{e_{2}}=f_{e^{ \pm}} u_{e_{1}}$ con lo cual la emisión de los primarios es también mayor que la de los secundarios y ajustamos el espectro observado con la emisión de los electrones acelerados en el choque reverso. Esto es, $p_{e_{1}}^{\prime}=2.18$ y fijando $p_{p}^{\prime}=p_{e_{1}}^{\prime}$ resulta $p_{e_{2}}^{\prime}=2.13$. Por otro lado, en el caso con $a=100$, resulta $u_{e_{2}} \sim 50 u_{e_{1}}$ y así el espectro observado se ajusta con la emisión sincrotrón producida por los pares. De esta manera, si $p_{e_{2}}^{\prime}=2.18$, entonces $p_{p}^{\prime}=2.27$ y por lo tanto $p_{e_{1}}^{\prime}=2.27$ también.

Fijados los valores de $p_{i}^{\prime}$ para cada caso y las relaciones entre las densidades de energía $u_{i}$, podemos determinar las constantes $k_{i}^{\prime}$. Para esto, nos valemos del flujo sincrotrón observado en radio, cuya expresión es la siguiente (Ginzburg \& Syrovatskii 1964):

$$
S_{\nu}=1.35 \times 10^{-22} a_{\mathrm{sin}}\left(p_{i}^{\prime}\right) \frac{k_{i}^{\prime} V_{\mathrm{lob}} B_{\mathrm{lob}}^{\left(p_{i}^{\prime}+1\right) / 2}}{d_{\mathrm{nm}}^{2}}\left(\frac{6.26 \times 10^{18}}{\nu}\right)^{\left(p_{i}^{\prime}-1\right) / 2},
$$




\begin{tabular}{ccccc}
\hline \hline $\mathrm{a}$ & $\begin{array}{c}B_{\mathrm{lob}} \\
{[\mathrm{G}]}\end{array}$ & $\begin{array}{c}k_{e_{1}}^{\prime} \\
{\left[\mathrm{erg}^{p_{e_{1}}^{-1}} \mathrm{~cm}^{-3}\right]}\end{array}$ & $\begin{array}{c}k_{p}^{\prime} \\
{\left[\mathrm{erg}^{p_{p}^{\prime}-1} \mathrm{~cm}^{-3}\right]}\end{array}$ & $\begin{array}{c}k_{e_{2}}^{\prime} \\
{\left[\mathrm{erg}^{p_{\mathrm{e}_{2}}^{-1}} \mathrm{~cm}^{-3}\right]}\end{array}$ \\
\hline 0 & $2.0 \times 10^{-3}$ & $2.6 \times 10^{-9}$ & - & - \\
1 & $2.5 \times 10^{-3}$ & $2.0 \times 10^{-9}$ & $6.7 \times 10^{-9}$ & $6.5 \times 10^{-10}$ \\
100 & $3.0 \times 10^{-3}$ & $2.7 \times 10^{-11}$ & $1.7 \times 10^{-8}$ & $1.5 \times 10^{-9}$ \\
\hline
\end{tabular}

Tabla 4.2: Campo magnético y constantes de normalización $\left(k_{i}^{\prime}\right)$ para los diferentes casos considerados en esta tesis.

donde $a_{\sin }\left(p_{i}^{\prime}\right)$ es una función complicada tabulada para diferentes valores de $p_{i}^{\prime}$. Para $p_{i}^{\prime}=2.18$ resulta $a_{\sin } \sim 0.1$, interpolando linealmente entre $a_{\sin }(2)$ y $a_{\sin }(2.5)$. El volumen del lóbulo sur es $V_{\text {lob }} \sim 5.6 \times 10^{48} \mathrm{~cm}^{3}$ y $d_{\mathrm{nm}}$ es la distancia a la fuente. Considerando que $S(\nu=8.46 \mathrm{GHz})=2.8 \mathrm{mJy}$ (Garay et al. 2003) y la ecuación (4.4) de equipartición de la energía es posible obtener $B_{\text {lob }}$ y $k_{i}^{\prime}\left(i=e_{1}\right.$ ó $\left.i=e_{2}\right)$ para cada valor del parámetro $a$. Luego, teniendo en cuenta las relaciones entre las densidades de energía $\left(u_{p}=a u_{e_{1}} \mathrm{y}\right.$ $\left.u_{e_{2}} \sim f_{e^{ \pm}} u_{p}\right)$, hallamos $k_{p}^{\prime}$ y la constante leptónica que falta. En la Tabla 4.2 se listan los valores de $B_{\mathrm{lob}}, k_{e_{1}}^{\prime}, k_{p}^{\prime}$ y $k_{e_{2}}^{\prime}$ calculados para los 3 valores de $a$ considerados.

Conociendo el valor de $B_{\text {lob }}$ podemos estimar el tiempo de aceleración tanto para protones como para electrones primarios. Aunque no tenemos información sobre la geometría de $\vec{B}_{\text {lob }}$ en los lóbulos, aquí adoptamos el valor más conservativo para la eficiencia acelerativa $\left(\vec{B}_{\text {lob }} \| \vec{v}_{\mathrm{ch}}\right)$ y es por esto que consideramos la fórmula (2.59) para calcular el tiempo de aceleración, resultando

$$
t_{\mathrm{ac}}=1.4 \times 10^{10} \frac{E_{e_{1}, p}}{B_{\mathrm{lob}} v_{\mathrm{r}}^{2}} \sim 1.4 \times 10^{-6} \frac{E_{e_{1}, p}}{B_{\mathrm{lob}}} \mathrm{s},
$$

si consideramos que $v_{\mathrm{r}} \sim v_{\mathrm{j}} \sim 1000 \mathrm{~km} \mathrm{~s}^{-1}$.

Como vimos en el Capítulo 2, la aceleración continúa hasta que las partículas o se escapan del acelerador o bien se enfrían por pérdidas radiativas. En el primer caso, el tiempo de escape $t_{\text {esc }}$ está dado por la ecuación (2.63), siendo el tiempo de convección

$$
t_{\mathrm{conv}} \sim \frac{R_{\mathrm{lob}}}{v_{\mathrm{bs}} / 4} \sim 4 \times 10^{8} \mathrm{~s},
$$

y el tiempo de difusión en el régimen de Bohm resulta

$$
t_{\mathrm{dif}} \sim 4.8 \times 10^{-20} \frac{B_{\mathrm{lob}} R_{\mathrm{lob}}^{2}}{E_{i}} \sim 5.8 \times 10^{6} \frac{B_{\mathrm{lob}}}{E_{i}} \mathrm{~s} .
$$

Además de acelerarse y/o escaparse, las partículas primarias y también los pares secundarios pueden perder energía a través de interacciones sucesivas con otras partículas o campos. 


\section{Pérdidas leptónicas}

Como vimos en el Capítulo 3, las interacciones de leptones relativistas con campos magnéticos producen radiación sincrotrón, cuyas pérdidas resultan (ver ecuación (3.15)):

$$
t_{\mathrm{sin}} \sim 4 \times 10^{2} \frac{1}{B_{\mathrm{lob}}^{2} E_{e_{1}, e_{2}}} \mathrm{~s} .
$$

Debido a que $B_{\text {lob }}$ cambia con $a$, para cada valor de este parámetro tenemos un valor diferente de $t_{\mathrm{sin}}$, al igual que de $t_{\mathrm{ac}}$.

Los leptones también pueden interactuar con los fotones IR producidos por la protoestrella masiva. La densidad de energía de estos fotones es $u_{\mathrm{ph}}=L_{\star} /\left(4 \pi R_{\mathrm{nm}}^{2} c\right)$, donde $L_{\star}$ es la luminosidad de la protoestrella y $R_{\mathrm{nm}}=D_{\mathrm{nm}} / 2$ es el radio de la nube. Resulta $u_{\mathrm{ph}} \sim 1.84 \times 10^{-9} \mathrm{erg} \mathrm{cm}^{-3}$ y los fotones correspondientes a esta densidad tienen una energía $E_{\mathrm{ph} \star} \sim 3 K_{\mathrm{B}} T_{\mathrm{nm}} \sim 6.6 \times 10^{-3} \mathrm{eV}$ al estar reprocesados por el polvo. Con fotones semilla de tan baja energía, las interacciones IC ocurrirán en el régimen de KN para energías tan altas como $E_{e_{1}, e_{2}}>\left(m_{e} c^{2}\right)^{2} / E_{\mathrm{ph} \star} \sim 3.8 \times 10^{13} \mathrm{eV}$. Tanto en el régimen de Th como en $\mathrm{KN}$ la fómula valida para el tiempo de enfriamiento es la ecuación (3.23), que en el caso del lóbulo sur nos queda

$$
t_{\mathrm{IC}} \sim 3.3 \times 10^{21} E_{\mathrm{ph} \star} \frac{(1+8.3 y)}{\ln (1+0.2 y)} \frac{\left(1+1.3 y^{2}\right)}{\left(1+0.5 y+1.3 y^{2}\right)} \mathrm{s},
$$

donde $y \equiv E_{\mathrm{ph} \star} E_{e_{1}, e_{2}} /\left(5.1 \times 10^{5} \mathrm{eV}\right)^{2}$.

Por otro lado, debido a que la nube tiene una densidad alta, $n_{\mathrm{nm}} \sim 5 \times 10^{5} \mathrm{~cm}^{-3}$, las pérdidas por Bremsstrahlung relativista serán importantes, con un tiempo característico (ver ecuación (3.32))

$$
t_{\mathrm{Brem}} \sim \frac{2.8 \times 10^{10}}{\ln \left(\frac{E_{e_{1}, e_{2}}}{m_{e} c^{2}}\right)+0.36} \mathrm{~s} .
$$

Para obtener la energía máxima que pueden alcanzar los electrones acelerados en el lóbulo sur, comparamos las ganancias por aceleración con las pérdidas radiativas. Para esto hemos graficado los tiempos definidos antes como se muestra en la Figura 4.5 (izquierda). Como vemos en el gráfico de la izquierda, los electrones alcanzan energías máximas $\sim 1 \mathrm{TeV}$, para el caso $a=1$, como consecuencia del balance entre la aceleración y las pérdidas por radiación sincrotrón. Como puede apreciarse también en la Figura 4.5 (izquierda), para $E_{e} \lesssim 3 \times 10^{10} \mathrm{eV}$, las pérdidas por sincrotrón dejan de dominar para hacerse más importantes aquellas por Bremsstrahlung relativista. A esta energía de quiebre, que llamamos $E_{\mathrm{q}}$, el espectro de electrones primarios sufre un cambio en su dependencia con $E_{e}$, ya que las pérdidas dominantes cambian. El valor de $E_{\mathrm{q}}$ puede determinarse igualando $t_{\text {sin }}=t_{\text {Brem }}$, con lo cual obtenemos $E_{\mathrm{q}} \sim 1.8 \times 10^{5} / B_{\text {lob }}^{2}$ eV. En la Tabla 4.4 listamos los valores de $E_{e_{1}}^{\max }$ y $E_{\mathrm{q}}$ para los tres valores de $a$ considerados.

Dado que el tiempo de vida de la fuente $\tau_{\text {vida }}\left(\sim t_{\mathrm{j}} \sim 10^{11} \mathrm{~s}\right)$ es mucho mayor que los tiempos de enfriamiento dominantes (sincrotrón y Bremsstrahlung relativista), el espectro 

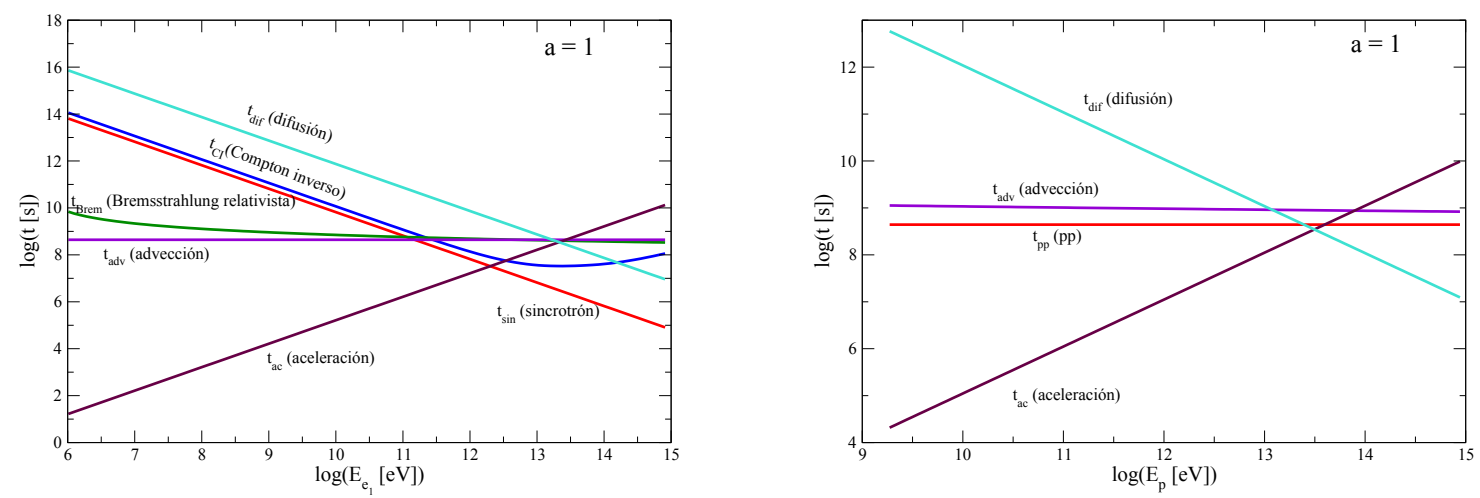

Figura 4.5: Tiempos de aceleración y de enfriamiento para los electrones primarios (izquierda) y para los protones (derecha). Estos gráficos corresponden al caso con $a=1$, pero al resto de los casos les corresponden figuras similares, ya que la única diferencia está en el valor de $B_{\text {lob }}$, que afecta a $t_{\mathrm{ac}}, t_{\mathrm{sin}} \mathrm{y} t_{\mathrm{dif}}$, y éste no cambia demasiado, como se muestra en la Tabla 4.2.

de energía de los electrones relativistas inyectados en el lóbulo se encuentra en el estado estacionario. Es decir, toda la energía de estas partículas relativistas se radía, básicamente, por emisión sincrotrón y Bremsstrahlung relativista dentro de la fuente (el lóbulo sur), ya que los tiempos radiativos son similares al tiempo de escape $\left(t_{\mathrm{esc}} \sim 4 \times 10^{8} \mathrm{~s}\right)$. Para mostrar esto hemos calculado el espectro de partículas resultante de la inyección $q_{e_{1}}\left(E_{e_{1}}\right)$, a diferentes tiempos de inyección $\tau_{\text {iny }}$. Considerando $\tau_{\text {iny }}=10^{7}, 10^{8}, 10^{9}$ y $10^{10}$ s obtuvimos los resultados que se muestran en la Figura 4.6. Finalmente, el espectro de electrones relativistas en el lóbulo sur resulta

$$
n_{e}\left(E_{e}\right)= \begin{cases}q_{e}\left(E_{e}\right) t_{\mathrm{Brem}} & \text { si } E_{e} \leq E_{\mathrm{q}} \\ q_{e}\left(E_{e}\right) t_{\mathrm{sin}} & \text { si } E_{e} \geq E_{\mathrm{q}}\end{cases}
$$

\section{Pérdidas hadrónicas}

En el caso de los protones, éstos pierden energía principalmente por interacciones con los protones fríos de la nube molecular, cuya densidad es $n_{\mathrm{nm}}$ y el tiempo de enfriamiento por $p p$ resulta:

$$
t_{p p} \sim 4.3 \times 10^{9} \mathrm{~s}
$$

Como se muestra en la Figura 4.5 (derecha), las pérdidas por difusión son importantes solo a energías muy altas, cercanas a $E_{p}^{\max }$. Esta última queda determinada entonces comparando el tiempo de aceleración con el de difusión, resultando $E_{p}^{\max } \sim 1.3 \times 10^{18} B_{\text {lob }}^{2} \mathrm{eV}$, como se muestra en la Tabla 4.4. 

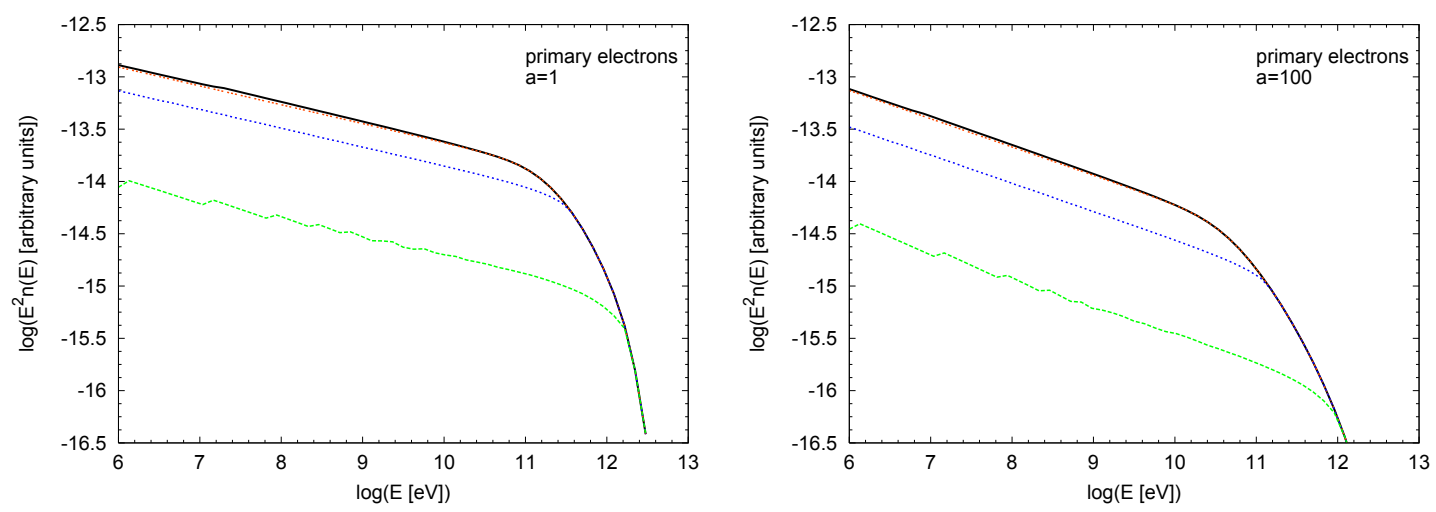

Figura 4.6: Distribuciones de energía de los electrones primarios. En cada panel mostramos las distribuciones para diferentes tiempos de inyección de partículas $\left(\tau_{\text {inj }}\right)$. Las diferentes curvas corresponden a los siguientes valores de $\tau_{\text {inj }}: 10^{7} \mathrm{~s}$ (línea de rayas largas, verde), $10^{8} \mathrm{~s}$ ( línea de rayas y puntos, azul), $10^{9} \mathrm{~s}$ (línea punteada, rojo) and $10^{10} \mathrm{~s}$ (línea sólida, negro). Notar que para edades $>10^{9} \mathrm{~s}$ el estado estacionario es alcanzado (Araudo et al. 2007).

\begin{tabular}{cccc}
\hline \hline $\mathrm{a}$ & $\begin{array}{c}E_{\mathrm{q}} \\
{[\mathrm{eV}]}\end{array}$ & $\begin{array}{c}k_{e_{1}, \mathrm{q}}^{\prime} \\
{\left[\mathrm{erg}^{p_{e_{1}}} \mathrm{~cm}^{-3}\right]}\end{array}$ & $\begin{array}{c}k_{e_{2}, \mathrm{q}}^{\prime} \\
{\left[\mathrm{erg}^{p_{e_{2}}^{\prime}} \mathrm{cm}^{-3}\right]}\end{array}$ \\
\hline 0 & $9.4 \times 10^{9}$ & $3.7 \times 10^{-11}$ & - \\
1 & $3.1 \times 10^{10}$ & $9.8 \times 10^{-11}$ & $3.2 \times 10^{-11}$ \\
100 & $2.2 \times 10^{10}$ & $9.5 \times 10^{-13}$ & $5.4 \times 10^{-11}$ \\
\hline
\end{tabular}

Tabla 4.3: Energía de quiebre del espectro de electrones para los diferentes casos discutidos en esta tesis. Las constantes de normalización $k_{i}^{\prime}\left(i=e_{1}, e_{2}\right)$ de las distribuciones de energía de los leptones para $E_{e_{1}, e_{2}}>E_{\mathrm{q}}$ están también listadas.

Considerando que el tiempo más relevante es $t_{p p}$, cuya dependencia con $E_{p}$ es despreciable (ver la Figura 4.5), el espectro de los protones relativistas resulta estacionario y con una dependencia con $E_{p}$ similar a la de la inyección, es decir

$$
n_{p}\left(E_{p}\right) \sim q_{p}\left(E_{p}\right) t_{p p} \propto E_{p}^{-p_{p}}
$$

Las interacciones $p p$ dan lugar a la producción de pares $e^{ \pm}$, los cuales radían luego por los mismos procesos leptónicos descriptos en la sección anterior. Como en el caso de los electrones primarios, hemos calculado la evolución temporal del espectro de los pares obteniendo que para $\tau_{\text {vida }} \sim 10^{11}$ s el espectro se encuentra en el estado estacionario. En la Figura 4.7 se muestran los resultados hallados. 


\begin{tabular}{cccc}
\hline \hline $\mathrm{a}$ & $\begin{array}{c}E_{\mathrm{e}_{1}}^{\max } \\
{[\mathrm{eV}]}\end{array}$ & $\begin{array}{c}E_{\mathrm{p}}^{\max } \\
{[\mathrm{eV}]}\end{array}$ & $\begin{array}{c}E_{\mathrm{e}_{2}}^{\max } \\
{[\mathrm{eV}]}\end{array}$ \\
\hline 0 & $3.1 \times 10^{12}$ & - & - \\
1 & $3.1 \times 10^{12}$ & $4.7 \times 10^{13}$ & $1.1 \times 10^{12}$ \\
100 & $2.8 \times 10^{12}$ & $5.7 \times 10^{13}$ & $1.8 \times 10^{12}$ \\
\hline
\end{tabular}

Tabla 4.4: Energías máximas obtenidas para los electrones y protones acelerados en el lóbulo sur. Además, las energías máximas de los pares secundarios se muestran en la última columna.
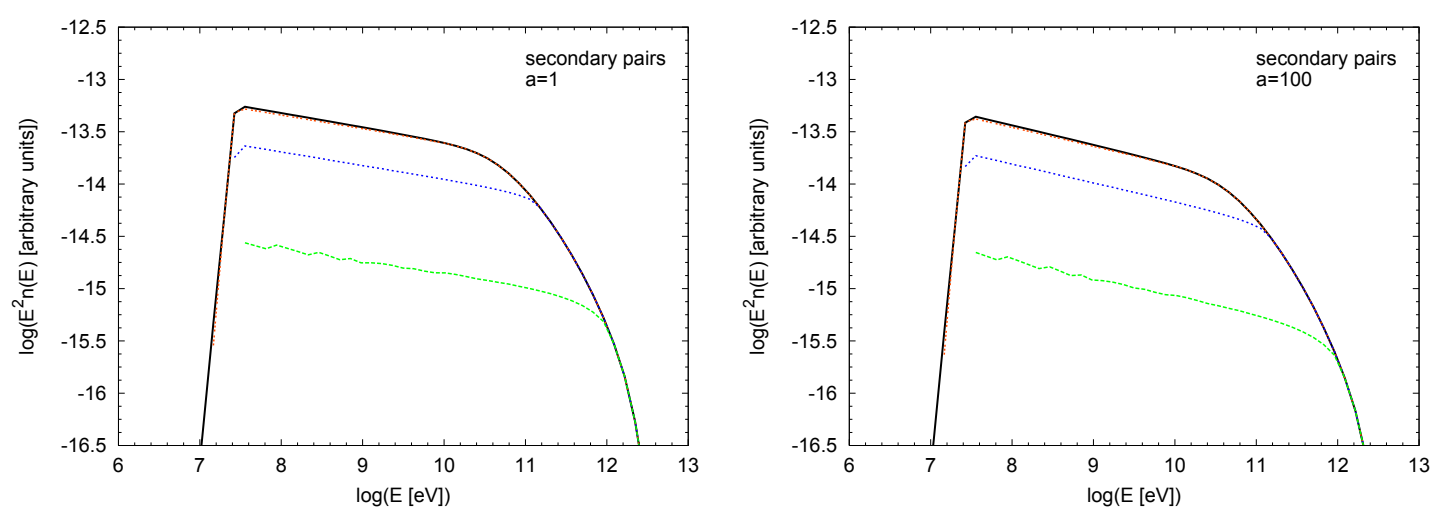

Figura 4.7: Distribuciones de energía de los pares secundarios. En cada panel mostramos las distribuciones para diferentes tiempos de inyección de partículas $\left(\tau_{\text {iny }}\right)$. Las diferentes curvas corresponden a los siguientes valores de $\tau_{\text {iny }}: 10^{7} \mathrm{~s}$ (línea de rayas largas, verde), $10^{8}$ $\mathrm{s}$ ( línea de rayas y puntos, azul), $10^{9} \mathrm{~s}$ (línea punteada, rojo) y $10^{10} \mathrm{~s}$ (línea sólida, negro). Notar que para edades $>10^{9} \mathrm{~s}$ el estado estacionario es alcanzado. La energía mínima de los pares $e^{ \pm}$inyectados en el lóbulo sur es $\sim 5 \times 10^{7} \mathrm{eV}$, como se aprecia en las figuras (Araudo et al. 2007). 


\subsection{Distribuciones espectrales de energía}

En las secciones previas vimos que en los choques terminales (en particular el choque reverso, en el caso de la fuente que estamos estudiando) se pueden acelerar partículas hasta energías $\gtrsim 10^{13} \mathrm{eV}$ y luego éstas se enfrían eficientemente por diversos procesos radiativos no térmicos. En esta sección calculamos las SEDs producidas por estos procesos radiativos, teniendo en cuenta las distribuciones $n_{i}\left(E_{i}\right)$ estimadas anteriormente y los campos magnéticos, de materia y de fotones presentes en el lóbulo sur de la fuente IRAS 165474247.

Asumiendo que el emisor es homogéneo y esférico con un radio $R_{\text {lob }}$ y volumen $V_{\text {lob }}$, consideraremos en todos los casos que la luminosidad específica es

$$
E_{\mathrm{ph}} L_{\mathrm{ph}}\left(E_{\mathrm{ph}}\right)=V_{\mathrm{lob}} E_{\mathrm{ph}} \int j_{\mathrm{ph}}\left(E_{\mathrm{ph}}, E_{i}\right) \mathrm{d} E_{i}
$$

donde $j_{\mathrm{ph}}\left(E_{\mathrm{ph}}, E_{i}\right)$ es la emisividad de cada proceso radiativo.

\subsubsection{Interacciones leptónicas}

Considerando los valores de $B_{\text {lob }}$ mostrados en la Tabla 4.2 calculamos la luminosidad específica emitida en el lóbulo sur por radiación sincrotrón. Por otro lado, con los valores de $u_{\mathrm{ph}} \mathrm{y} n_{\mathrm{nm}}$ calculamos las emisividades de los procesos IC y Bremsstrahlung relativista usando las fórmulas descriptas en el Capítulo 3. En la Figura 4.8 se muestra la SED correspondiente al caso puramente leptónico, $a=0$.

Como se muestra en la figura antes mencionada, la radiación a energías altas está dominada por la emisión producida por el mecanismo Bremsstrahlung relativista, con un pico de $\sim 10^{32} \mathrm{erg} \mathrm{s}^{-1}$ a $E_{\mathrm{ph}} \sim 1 \mathrm{MeV}$. A energías $E_{\mathrm{ph}} \gtrsim 1 \mathrm{GeV}$, la fuente presenta luminosidades de $\sim 10^{31} \mathrm{erg} \mathrm{s}^{-1}$, con un cut-off a $E_{\mathrm{ph}} \sim 10 \mathrm{GeV}$. En rayos X, en el rango 1-10 $\mathrm{keV}$, las luminosidades esperadas son de $\sim 10^{30} \mathrm{erg} \mathrm{s}^{-1}$, presentando un ablandamiento del espectro debido al cut-off exponencial $\exp \left(-E_{e_{1}} / E_{e_{1}}^{\max }\right)$ de la distribución de los electrones primarios. En las energías más bajas, el espectro calculado (sincrotrón) ajusta muy bien los puntos observados a las frecuencias $\nu=8.4$ y $14.9 \mathrm{GHz}$.

\subsubsection{Interacciones hadrónicas}

En los casos con $a=1$ y $a=100$ la emisión de los protones es significativa, ya que la densidad $n_{\mathrm{nm}}$ de la nube molecular es alta. Calculando la emisividad $p p$ a través de la fórmula (3.39) obtenemos las luminosidades específicas que se muestran en la Figura 4.9. además de las leptónicas (sincrotrón, IC y Bremsstrahlung relativista). En el caso con $a=1$ la luminosidad emitida por $p p$ es similar a la emitida por Bremsstrahlung relativista $\left(\sim 10^{32} \mathrm{erg} \mathrm{s}^{-1}\right)$ pero la primera se extiende hasta energías más altas, $\sim 1 \mathrm{TeV}$. Los leptones primarios dominan la emisión por sincrotrón y por Bremsstrahlung relativista en el caso $a=1$, mientras que en el caso con $a=100$ la contribución más importante a la SED es producida por los pares secundarios. En ningún caso la emisión por IC es significativa. 


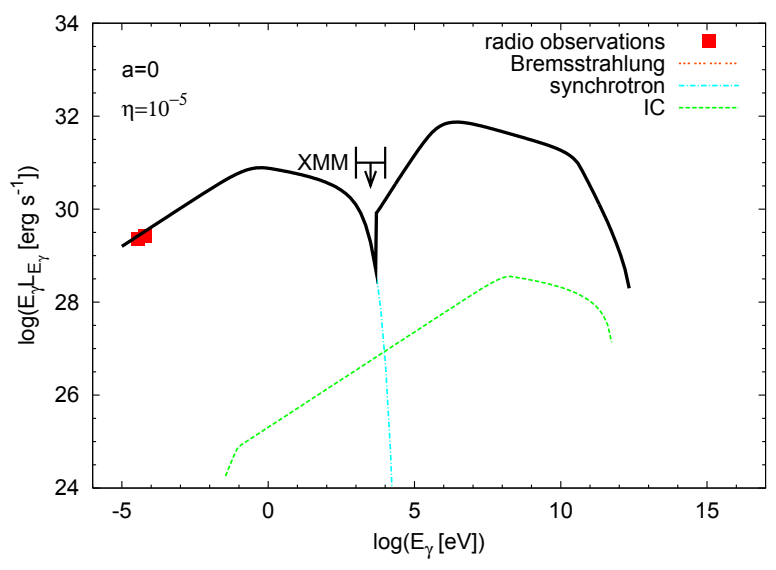

Figura 4.8: Distribución espectral de energía para el caso puramente leptónico, $a=0$. La línea negra representa la suma de las tres contribuciones (sincrotrón, IC y Bremsstrahlung relativista) a la SED total. En las frecuencias $\nu=8.4$ y $14.9 \mathrm{GHz}\left(E_{\mathrm{ph}} \sim 3.5 \times 10^{-5} \mathrm{y}\right.$ $6.2 \times 10^{-5} \mathrm{eV}$, respectivamente) se representa con cuadrados rojos la luminosidad observada con VLA a estas frecuencias. En el rango de los rayos X se muestra la sensibilidad del satélite $X M M$, mostrando que nuestra predicción teórica está de acuerdo con el hecho de que este instrumento no haya detectado a la fuente (Araudo et al. 2007).

Básicamente la emisión para $E_{\mathrm{ph}}>1 \mathrm{GeV}$ es debida al decaimiento de los $\pi^{0}$, mientras que la emisión en rayos $\mathrm{X}$ y en rayos gamma blandos es producida por Bremsstrahlung relativista. Para los casos $a=1$ y $a=100$, las componentes espectrales debidas a la radiación sincrotrón y Bremsstrahlung relativista de los pares secundarios tienen la misma forma que la emisión de los primarios (para los mismos casos) pero diferentes a bajas energías debido a que la energía mínima de cada distribución de partículas (electrones primarios y pares secundarios) es distinta. En el caso de los electrones primarios la energía mínima considerada es $\sim 2 m_{e} c^{2}$ mientras que los pares $e^{ \pm}$tienen una energía mínima determinada por la energía umbral para la creación de los piones cargados: $E_{\mathrm{u}} \sim 1.22 \mathrm{GeV}$.

\subsection{Discusión}

Las fuentes de rayos gamma del plano galáctico son usualmente asociadas con regiones de formación estelar (Romero et al. 1999). Sin embargo las fuentes detectadas por EGRET2 son mucho más luminosas que los YSOs de gran masa, de acuerdo a los cálculos realizados con nuestro modelo. Las fuentes EGRET no identificadas tendrían como contrapartidas a púlsares, remanentes de supernovas, estrellas tempranas (Romero 2001) y MQs (BoschRamon et al. 2005). La emisión producida por YSOs de gran masa está por debajo de

\footnotetext{
${ }^{2}$ El instrumento EGRET (a bordo del satélite Compton) funcionó en los años 1991-2000 y detectaba fotones con energías $30 \mathrm{MeV} \lesssim E_{\mathrm{ph}} \lesssim 300 \mathrm{GeV}$. Con los datos obtenidos se confeccionó el primer catálogo de fuentes de rayos gamma que permitió realizar estudios poblacionales.
} 

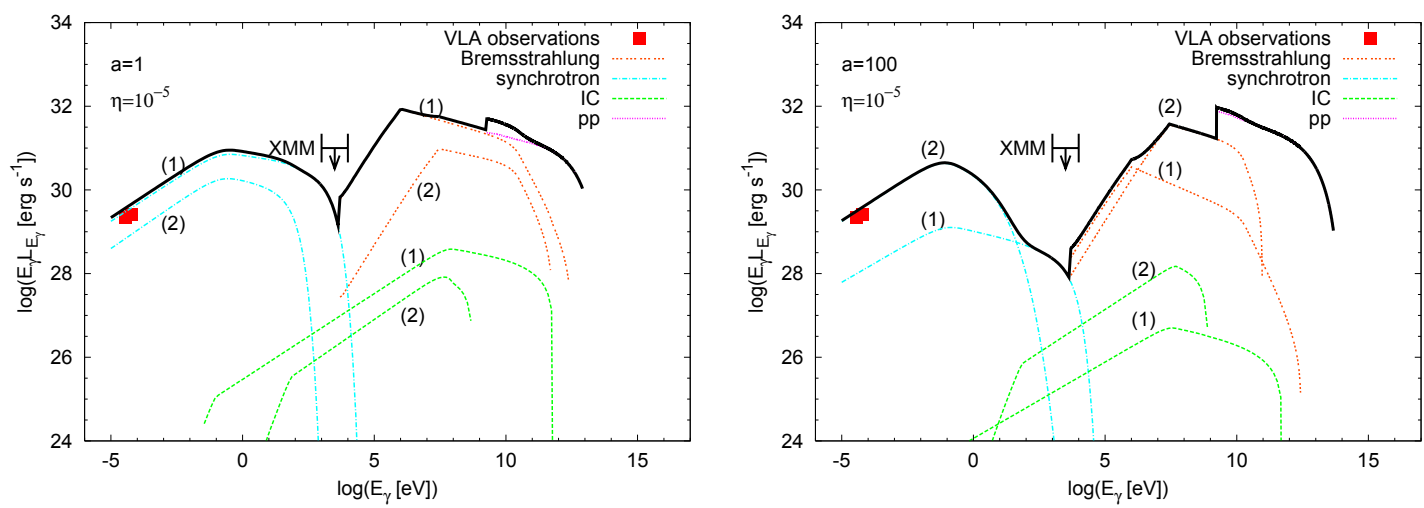

Figura 4.9: Distribuciones espectrales de energía para los casos con $a=1$ (izquierda) y 100 (derecha), en los cuales consideramos tanto una población leptónica como hadrónica de partículas primarias y la población de pares $e^{ \pm}$producida. Con (1) y (2) indicamos la contribución de los leptones primarios y secundarios, respectivamente. Como mencionamos en la Figura 4.8, la línea negra indica la SED total (suma de todas las contribuciones, leptónicas y hadrónica) y los cuadrados rojos indican la luminosidad observada con VLA. La sensibilidad de $X M M$ es también graficada mostrando nuevamente que los límites observacionales en rayos X tampoco se violan en los casos con $a=1$ y 100 (Araudo et al. 2007).

la sensibilidad de EGRET, pero éstos podrían ser detectados por Fermi. En el rango de las VHE, los futuros arreglos de telescopios Cherenkov como HESS II, MAGIC II y CTA 3 podrían detectar fuentes como IRAS 16547-4247. Ya que estos instrumentos deberían llegar a medir hasta el cut-off de altas energías, la medición de este valor daría una valiosa información acerca de la eficiencia de aceleración de partículas en los choques terminales de los jets de YSOs de gran masa.

En rayos X, la fuente debería ser detectable por Chandra y XMM-Newton, probablemente como una fuente puntual a través de una observación profunda en la región. En los datos de archivo de XMM-Newton, no se ve una emisión significativa por encima de la radiación de fondo en la región donde la fuente IRAS está localizada. Esto nos proporciona una cota máxima de $\sim 10^{31} \mathrm{erg} \mathrm{s}^{-1}$ (a la distancia $\sim 2.9 \mathrm{kpc}$ ) en el rango de 1 a $10 \mathrm{keV}$ a las luminosidades que obtenemos con nuestros modelos teóricos, como se muestra en las Figuras 4.8 y 4.9. Sin embargo, una observación más profunda (más de 30 ks) debería detectar radiación por encima de la emisión de fondo de esta región. Si las temperaturas del material chocado son suficientemente altas, la componente térmica debería ser también detectable en rayos X. En la banda ultravioleta (UV), esta emisión térmica podría ser aún

\footnotetext{
${ }^{3}$ Los instrumentos HESS II y MAGIC II serían los que reemplazarían a HESS y MAGIC, respectivamente. Por otro lado, los instrumentos que formarán parte del arreglo de telescopios Cherenkov CTA (por Cherenkov Telescope Array) ya comenzaron a contruirse, aunque el sitio donde estarán montados aún no esta definido.
} 
mayor que la producida por las partículas no térmicas, pero sería difícil de detectant

Un hecho relevante respecto de la factibilidad del escenario discutido en este capítulo es que las luminosidades no térmicas son bastante más bajas que la luminosidad cinética del jet de la fuente IRAS 16547-4247. La pérdida de masa del jet ha sido estimada en $\sim 10^{-5} M_{\odot} \mathrm{yr}^{-1}$ y con una velocidad $v_{\mathrm{j}} \sim 1000 \mathrm{~km} \mathrm{~s}^{-1}$ con lo que la luminosidad cinética del jet resulta $\sim 10^{36} \mathrm{erg} \mathrm{s}^{-1}$, más de tres órdenes de magnitud mayor que la luminosidad no térmica predicha por nuestro modelo. Esto es, nuestro modelo predice niveles de emisión que respetan los límites energéticos de la fuente. Por lo tanto, todavía sería posible un incremento de la eficiencia radiativa, incrementando así las posibilidades de detectección de este tipo de objetos. Además de la fuente IRAS 16547-4247, otras protoestrellas de gran masa son también potenciales candidatos a fuentes de rayos gamma. Entre ellas podemos mencionar HH 80-81 (Bosch-Ramon et al. 2010) y W3(OH) (Araudo et al. 2008a).

\footnotetext{
${ }^{4}$ Para una discusión más detallada sobre la emisión térmica, como así también un análisis más preciso del impacto del medio en la evolución de las partículas y en la radiación producida, ver Bosch-Ramon et al. (2010).
} 


\section{Capítulo 5}

\section{Microcuasares}

\subsection{Introducción}

Las estrellas en general no se encuentran aisladas, sino que la mayoría de ellas forman sistemas binarios o cúmulos. En los primeros, si ambas componentes tienen masas diferentes, la estrella más temprana evolucionará más rápido que la más tardía y así las estrellas estarán en diferentes estados evolutivos. Si la masa de la estrella más evolucionada que queda luego de la explosión de supernova es $3 M_{\odot} \gtrsim M_{\star} \gtrsim 1.4 M_{\odot}$, entonces el objeto remanente al final de la evolución será una estrella de neutrones, mientras que si $M_{\star} \gtrsim 3 M_{\odot}$ será un agujero negro.

Consideremos un sistema binario formado por una estrella no degenerada, la cual puede estar en diferentes estados de su evolución, y el objeto colapsado, el cual puede ser un agujero negro o una estrella de neutrones. Cuando la estrella compañera se convierte en gigante roja, las capas externas de la atmósfera de la misma llenan de material el lóbulo de Roche. La materia aquí dentro se escapa a través del punto lagrangiano L1 siendo acretada por el objeto compacto. La materia cae a la superficie del objeto a través de un disco de acreción debido a la conservación del momento angular. La materia en el disco rota a una velocidad que disminuye con la distancia $r_{\mathrm{d}}$ al objeto compacto. Anillos a diferentes $r_{\mathrm{d}}$ rotan a velocidades distintas lo que produce disipación de energía y calentamiento por roce de anillos contiguos. La materia del disco se puede calentar hasta temperaturas suficientemente altas como para radiar térmicamente en rayos $\mathrm{X}$.

Los sistemas binarios que emiten fuertemente en rayos $\mathrm{X}$ debido a la acreción, son llamados binarias de rayos X (XRB, por X-Ray Binaries). Si la estrella primaria es de gran masa (tipo espectral $\mathrm{O}$ ó $\mathrm{B}, M_{\star}>8 M_{\odot}$ ) entonces el sistema se dice de gran masa (HMXB, por High Mass X-ray Binary), mientras que si es vieja el sistema binario es una binaria de rayos X de baja masa (LMXB, por Low Mass X-ray Binary). Una subclase de las XRB son los MQs, los cuales presentan jets extendidos en la banda de radio (Mirabel \& Rodríguez 1999). Estos jets son flujos colimados de materia que se mueve a una velocidad de conjunto relativista. La materia que forma los jets puede ser materia arrancada del disco de acreción por las líneas de campo magnético (Blandford \& Payne 1981) o bien 


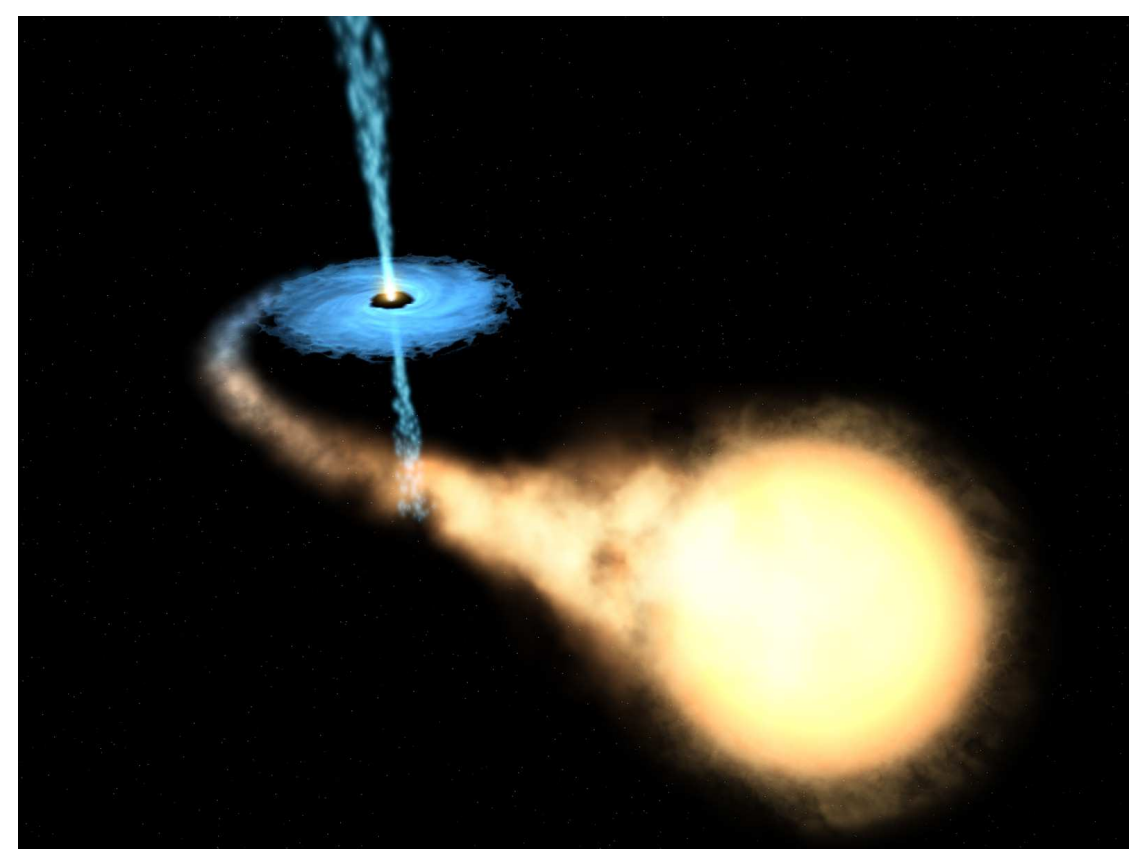

Figura 5.1: Esquema de un MQ. Se muestra una estrella evolucionada transfiriendo masa al objeto compacto a través del disco de acreción. Jets bipolares emergen del objeto compacto. (Crédito: ESA, NASA y Felix Mirabel.)

pares $e^{ \pm}$generados en la ergosfera del agujero negro (Blandford \& Znajek 1977) y lanzados en forma de chorros por procesos MHDs. El campo magnético juega un rol importante en la formación y colimación de los jets.

Se ha detectado emisión no térmica proveniente de los jets de los MQs. Esta emisión abarca desde las frecuencias radio (Ribó 2005) hasta los rayos X (Corbel et al. 2002), aunque emisión de más alta energía (rayos gamma) también puede producirse (Kaufmann Bernadó et al. 2002, Bosch-Ramon et al. 2006). Los telescopios MAGIC y AGILE han detectado emisión transitoria (flares) en HE y VHE asociada a los HMMQs Cygnus X-3 (Tavani et al. 2009b; Abdo et al. 2009a) y Cygnus X-1 (Albert et al. 2007; Sabatini et al. 2010), respectivamente. Eventos de emisión transitoria en rayos gamma también han sido observados en las HMXBs LS 5039 y LS I+61 303 por los telescopios HESS (Aharonian et al. 2005) y MAGIC (Albert et al. 2006), respectivamente, aunque la naturaleza de las fuentes no ha sido aún confirmada en estos casos. (Una discusión sobre el tema puede encontrarse en Romero et al. 2007.) Por otro lado, el satélite Fermi ha detectado emisión esporádica proveniente de fuentes no identificadas del plano galáctico (ATe1 1394, Abdo et al. 2009). Esta emisión altamente variable detectada en rayos gamma y generada en

\footnotetext{
${ }^{1}$ La sigla ATel (por Astronomical Telegrams) se utiliza para designar los reportes sobre descubrimientos observacionales. La página de internet en la cual pueden hallarse estos reportes es http://www.astronomerstelegram.org/.
} 
HMMQs podría tener un origen similar. Por ejemplo, la interacción entre el jet y el viento de la estrella compañera podría producir flares en el rango de las HE y VHE.

La pérdida de masa de las estrellas de gran masa forma un viento estelar que se propaga a velocidades supersónicas. Observaciones en rayos X de las líneas de emisión producidas en el viento indican que éste no tiene una estructura uniforme sino porosa, con clumps (o grumos de materia) que se forman a una distancia del orden de un radio estelar $\left(R_{\star}\right)$ de la superficie de la estrella (e.g. Puls et al. 2006, Owocki \& Cohen 2006). Sin embargo, las características de estos clumps no se conocen ya que la resolución espacial de los telescopios aún no es suficiente. Por esto las propiedades de los clumps, como el tama no, la densidad y el factor de llenado, no son bien conocidas y se estudian a través de métodos indirectos como el análisis de líneas espectroscópicas (Moffat 2008).

En este capítulo proponemos un modelo para explicar los flares en rayos gamma detectados en algunas binarias de gran masa, basado en la interacción de los jets del HMMQ con las inhomogeneidades del viento de la estrella compañera. Los clumps pueden eventualmente penetrar en el jet y producir emisión no térmica transitoria, generada al convertir parte de la energía cinética del jet en energía de partículas relativistas, las cuales emitirán radiación sincrotrón, IC y sufrirán colisiones $p p$.

\subsection{Escenario}

Para estudiar la interacción entre un clump del viento estelar con uno de los jets de un HMMQ adoptamos un escenario con características similares a las del sistema binario Cygnus X-1. Fijamos la separación $a_{\mathrm{mq}}$ entre el objeto compacto y la estrella masiva en $3 \times 10^{12} \mathrm{~cm}(\sim 0.2 \mathrm{UA})$. Para la luminosidad y la temperatura de la estrella adoptamos los siguientes valores: $L_{\star}=10^{39} \mathrm{erg} \mathrm{s}^{-1}$ y $T_{\star}=3 \times 10^{4} \mathrm{~K}$, respectivamente. Por otro lado suponemos que la pérdida de masa de la estrella es $\dot{M}_{\star}=3 \times 10^{-6} M_{\odot} \mathrm{yr}^{-1}$ con una velocidad terminal del viento $v_{\mathrm{v}} \sim 2.5 \times 10^{8} \mathrm{~cm} \mathrm{~s}^{-1}$. Un esquema gráfico de este escenario se muestra en la Figura 5.2 .

\section{Modelo para el clump}

Debido a las incertezas en la determinación de las características de los clumps, suponemos que éstos son esféricos y consideramos dos valores para el radio: $R_{\mathrm{c}}=10^{10}$ y $10^{11} \mathrm{~cm}\left(\sim 3 \times 10^{-3}-3 \times 10^{-2} a_{\mathrm{mq}}\right)$. A su vez adoptamos una distribución uniforme de densidad en el clump, $n_{\mathrm{c}}=10^{12} \mathrm{~cm}^{-3}$, que corresponde a un factor de llenado $f_{\mathrm{c}}=\dot{M}_{\star} / 4 \pi a_{\mathrm{mq}}^{2} m_{p} v_{\mathrm{c}} n_{\mathrm{c}} \sim 0.005$, donde $v_{\mathrm{c}}$ es la velocidad de los clumps y consideramos que éstos se mueven a la velocidad del viento, esto es, $v_{\mathrm{c}}=v_{\mathrm{v}}$ (Owocki et al. 2009 y referencias en ese artículo). La temperatura de los clumps, $T_{\mathrm{c}}$, es fijada en $10^{4} \mathrm{~K}$ (Krtička \& Kubát 2001), siendo moderadamente menor que la temperatura superficial de la estrella primaria. 


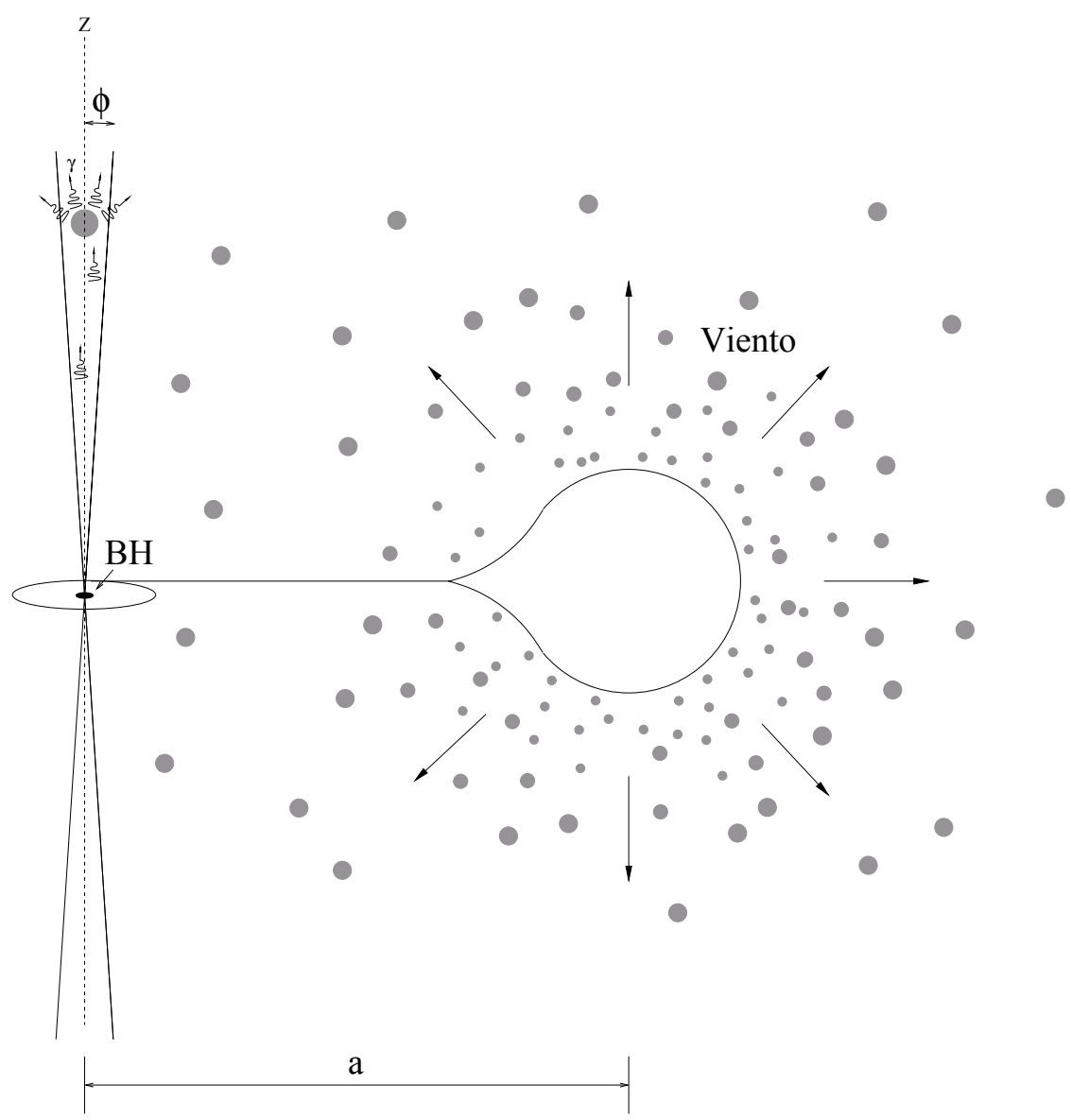

Figura 5.2: Esquema de un MQ. El viento de la estrella compañera presenta clumps, algunos de los cuales pueden llegar a penetrar en el jet (Romero et al. 2008). 
Tabla 5.1: Parámetros adoptados y estimados para el escenario en el cual desarrollamos el estudio presentado en este capítulo.

\begin{tabular}{lll}
\hline \hline Parámetro [unidades] & Clump & Jet \\
\hline Radio $[\mathrm{cm}]$ & $R_{\mathrm{c}}=10^{10}-10^{11}$ & $R_{\mathrm{j}}=1.5 \times 10^{11}$ \\
Velocidad $\left[\mathrm{cm} \mathrm{s}^{-1}\right]$ & $v_{\mathrm{c}}=2.5 \times 10^{8}$ & $v_{\mathrm{j}}=10^{10}$ \\
Densidad $\left[\mathrm{cm}^{-3}\right]$ & $n_{\mathrm{c}}=10^{12}$ & $n_{\mathrm{j}}=4.7 \times 10^{7}$ \\
\hline & Sistema binario & \\
\hline Tamaño del sistema $[\mathrm{cm}]$ & $a_{\mathrm{mq}}=3 \times 10^{12}$ & \\
Luminosidad de la estrella $\left[\mathrm{erg} \mathrm{s}^{-1}\right]$ & $L_{\star}=10^{39}$ & \\
Temperatura de la estrella $[\mathrm{K}]$ & $T_{\star}=3 \times 10^{4}$ & \\
Tasa de pérdida de masa $\left[\mathrm{M}_{\odot} \mathrm{yr}^{-1}\right]$ & $\dot{M}_{\star}=3 \times 10^{-6}$ & \\
Velocidad del viento $\left[\mathrm{cm} \mathrm{s}^{-1}\right]$ & $v_{\mathrm{v}}=2.5 \times 10^{8}$ & \\
\hline
\end{tabular}

\section{Modelo para el jet}

Para los jets, adoptamos un modelo hidrodinámico, es decir, consideramos un jet dominado dinámicamente por protones fríos con una velocidad de conjunto moderadamente relativista: $v_{\mathrm{j}}=0.3 c$, que corresponde a un factor de Lorentz $\Gamma_{\mathrm{j}}=1.05$. Observaciones en radio muestran que los jets de los MQs son extremadamente colimados (Miller-Jones et al. 2006). En esta tesis hemos supuesto que la relación entre el radio y la altura es $R_{\mathrm{j}}\left(z_{\mathrm{j}}\right)=0.1 z_{\mathrm{j}}$, lo que corresponde a un ángulo de apertura $\phi=6^{\circ}$. La velocidad de expansión del jet resulta $v_{\exp }=0.1 v_{\mathrm{j}}$, que para un jet hidrodinámico en expansión libre implica un número de Mach en la base de $\sim v_{\mathrm{j}} / v_{\exp } \sim 10$, con lo cual el jet es supersónico. La luminosidad cinética del jet es fijada en $L_{\mathrm{j}}=3 \times 10^{36} \mathrm{erg} \mathrm{s}^{-1}$, similar a la estimada para Cygnus X-1 (e.g. Gallo et al. 2005, Russell et al. 2007). Usando la ecuación (2.43) podemos estimar la densidad de partículas $n_{\mathrm{j}}$ del jet en el sistema de referencia del laboratorio. A la altura de la interacción jet-clump, que hemos fijado en $z_{\text {int }}=a_{\mathrm{mq}} / 2$, obtenemos $n_{\mathrm{j}}=4.7 \times 10^{7} \mathrm{~cm}^{-3}$. De esta manera, el cociente entre la densidad del clump y la del jet resulta $\chi_{\mathrm{mq}}=2.1 \times 10^{4}$. Finalmente mencionamos que despreciamos la curvatura del jet producida por la interacción con el viento estelar. En los jets de HMMQs con luminosidades cinéticas $>10^{36} \mathrm{erg} \mathrm{s}^{-1}$, la geometría de éstos no debería ser modificada considerablemente por el viento (Perucho \& Bosch-Ramon 2008), aunque este efecto puede ser importante en sistemas con jets tipo $\mathrm{HH}$ (protoestelares) interactuando con el viento de una estrella (Raga et al. 2009).

Los valores de los parámetros del jet y del clump que suponemos o estimamos en este capítulo están listados en la Tabla 5.1.

\subsubsection{Interacción jet-clump}

Los clumps se forman en la región de aceleración del viento de la estrella, aproximadamente a una distancia $\sim R_{\star}$ de la superficie de la misma (Puls et al. 2006). Algunos 
de estos clumps pueden llegar hasta el jet y penetrar en él, debido al gran contraste de densidades $\chi_{\mathrm{mq}}\left(\equiv n_{\mathrm{c}} / n_{\mathrm{j}}\right)$.

Habiendo especificado las características que adoptamos para los clumps y los jets de nuestro modelo, nos concentramos ahora en la interacción de uno de estos clumps con uno de los jets del HMMQ. De acuerdo a los parámetros considerados, las escalas de tiempo de los procesos dinámicos descriptos en la Sección 2.5.1 toman los valores que exponemos a continuación y que resumimos en la Tabla 5.2

El tiempo de penetración del clump en el jet es:

$$
t_{\mathrm{c}} \sim 80\left(\frac{R_{\mathrm{c}}}{10^{10} \mathrm{~cm}}\right)\left(\frac{v_{\mathrm{c}}}{2.5 \times 10^{8} \mathrm{~cm} \mathrm{~s}^{-1}}\right)^{-1} \mathrm{~s},
$$

mientras que el tiempo de cruce a una altura $z_{\text {int }}=a_{\mathrm{mq}} / 2=1.5 \times 10^{12} \mathrm{~cm}$ es

$$
t_{\mathrm{j}} \sim 1.2 \times 10^{3}\left(\frac{z_{\text {int }}}{1.5 \times 10^{12} \mathrm{~cm}}\right) \mathrm{s}
$$

El bow shock en el jet alcanza el estado estacionario en un tiempo muy corto. Para los parámetros de este sistema y considerando que $v_{\mathrm{j} 1} \sim v_{\mathrm{j}} / 4$ y que $Z=0.2 R_{\mathrm{c}}$ (van Dicke \& Gordon, 1959) tenemos que

$$
t_{\mathrm{bs}} \sim 0.8\left(\frac{R_{\mathrm{c}}}{10^{10} \mathrm{~cm}}\right)\left(\frac{v_{\mathrm{j}}}{10^{10} \mathrm{~cm} \mathrm{~s}^{-1}}\right)^{-1} \mathrm{~s} .
$$

El tiempo caracaterístico de este tipo de interacciones está dado, como mencionamos en el Capítulo 2. por aquel en el cual el choque que se propaga en el clump lo recorre completamente. Esta escala temporal está caracterizada de la siguiente manera en un HMMQ:

$$
\begin{aligned}
t_{\mathrm{cc}} \sim & 5 \times 10^{3}\left(\frac{R_{\mathrm{c}}}{10^{10} \mathrm{~cm}}\right)\left(\frac{n_{\mathrm{c}}}{10^{12} \mathrm{~cm}^{-3}}\right)^{1 / 2}\left(\frac{z_{\mathrm{int}}}{1.5 \times 10^{12} \mathrm{~cm}}\right) \\
& \times\left(\frac{\Gamma_{\mathrm{j}}-1}{0.06}\right)^{-1 / 2}\left(\frac{L_{\mathrm{j}}}{3 \times 10^{36} \mathrm{erg} \mathrm{s}^{-1}}\right)^{-1 / 2}\left(\frac{v_{\mathrm{j}}}{10^{10} \mathrm{~cm} \mathrm{~s}^{-1}}\right)^{-1 / 2} \mathrm{s.}
\end{aligned}
$$

La materia del jet acelera al clump aplicándole una fuerza $\sim M_{\mathrm{c}} \vec{g}$, donde $M_{\mathrm{c}}$ es la masa del clump y la aceleración $g \sim 3 \times 10^{4}$ y $3 \times 10^{3} \mathrm{~cm} \mathrm{~s}^{-2}$, para $R_{\mathrm{c}}=10^{10}$ y $10^{11} \mathrm{~cm}$, respectivamente. Esta fuerza puede acelerar al clump hasta la velocidad del jet en un tiempo

$$
\begin{aligned}
t_{\mathrm{g}} \sim & 1.3 \times 10^{6}\left(\frac{R_{\mathrm{c}}}{10^{10} \mathrm{~cm}}\right)\left(\frac{n_{\mathrm{c}}}{10^{12} \mathrm{~cm}^{-3}}\right)\left(\frac{z_{\text {int }}}{1.5 \times 10^{12} \mathrm{~cm}}\right)^{2} \\
& \times\left(\frac{\Gamma_{\mathrm{j}}-1}{0.06}\right)\left(\frac{L_{\mathrm{j}}}{3 \times 10^{36} \mathrm{erg} \mathrm{s}^{-1}}\right)^{-1} \mathrm{~s} .
\end{aligned}
$$


Tabla 5.2: Valores obtenidos para las escalas de tiempo descriptas en la sección 5.2.1, usando los valores de los parámetros listados en la Tabla 5.1 .

\begin{tabular}{c|cc}
\hline \hline Escala de tiempo $[\mathrm{s}]$ & $R_{\mathrm{c}}=10^{10} \mathrm{~cm}$ & $R_{\mathrm{c}}=10^{11} \mathrm{~cm}$ \\
\hline$t_{\mathrm{c}}$ & 80 & 800 \\
$t_{\mathrm{j}}$ & $1.2 \times 10^{3}$ & $1.2 \times 10^{3}$ \\
$t_{\mathrm{cc}}$ & $5 \times 10^{3}$ & $5 \times 10^{4}$ \\
$t_{\mathrm{bs}}$ & 0.8 & 8 \\
$t_{\mathrm{g}}$ & $7 \times 10^{10}$ & $7 \times 10^{11}$ \\
\hline
\end{tabular}

Sin embargo, antes de que el jet acelere al clump y éste comience a ser arrastrado por el flujo, el clump puede ser destruido por las inestabilidades de Rayleigh-Taylor y KelvinHelmholtz, las cuales crecen en un tiempo $t_{\mathrm{RT} / \mathrm{KH}} \sim t_{\mathrm{cc}} \ll t_{\mathrm{g}}$ hasta longitudes de escala $\sim R_{\mathrm{c}}$ (sin considerar el efecto estabilizador que puede llegar a tener el campo magnético).

A modo de resumen y de acuerdo a las escalas de tiempo estimadas en los párrafos previos, el clump puede penetrar completamente en el jet si $t_{\mathrm{c}}<t_{\mathrm{cc}}$. En nuestros cálculos radiativos no consideraremos los detalles de la penetración del clump en el jet, sino que para un tiempo $>t_{\mathrm{c}}$ el primero se encuentra completamente dentro del último (esto es, consideramos un sistema con simetría cilíndrica como se muestra en la Figura 5.31). El bow shock se forma rápidamente en un tiempo $t_{\mathrm{bs}}$ mucho menor que $t_{\mathrm{c}} \mathrm{y}$ que $t_{\mathrm{cc}}$. Por último, notamos que el clump podría no escapar del jet ya que para los parámetros considerados y el $z_{\text {int }}$ que hemos fijado, $t_{\mathrm{j}}>t_{\mathrm{RT} / \mathrm{KH}}$. Sin embargo, sólo podemos cuantificar el tiempo de las inestabilidades de una manera muy somera y no podemos afirmar que el clump se destruirá necesariamente antes de poder escapar del jet. Simulaciones numéricas muestran que las escalas de tiempo de las inestabilidades pueden ser varias veces el tiempo de cruce del choque en el clump, es decir, $t_{\mathrm{RT} / \mathrm{KH}}>t_{\mathrm{cc}}$ (Klein et al. 1994).

Respecto de las propiedades de los choques y dadas las características específicas de este escenario (HMMQ), el choque en el clump es fuerte, radiativo y lento, mientras que el bow shock es también fuerte pero adiabático y rápido. Por estas razones, el material chocado y calentado del clump radía una fracción significativa de la energía que el choque le ha transferido (pero debido a que $\chi_{\mathrm{mq}} \gg 1$, esta energía es baja comparada con la que transporta el jet).

\subsubsection{Emisión térmica del clump}

Para estimar la densidad $n_{\mathrm{c} 1}(x)$ y la temperatura $T_{\mathrm{c} 1}(x)$ del material del clump chocado a una distancia $x$ del choque usamos las relaciones (2.33) y (2.32) considerando que $\Lambda(T)=$ $7 \times 10^{-19} T^{-0.6} \mathrm{erg} \mathrm{cm}^{-3} \mathrm{~s}^{-1}$ y que la temperatura y la densidad en la zona adiabática de la región chocada del clump toman los valores $8.5 \times 10^{6} \mathrm{~K}$ y $4 \times 10^{13} \mathrm{~cm}^{-3}$, respectivamente. En la Figura 5.4 se muestran los gráficos de $n_{\mathrm{c} 1}(x)$ y $T_{\mathrm{c} 1}(x)$. Luego, el tiempo de enfriamiento 


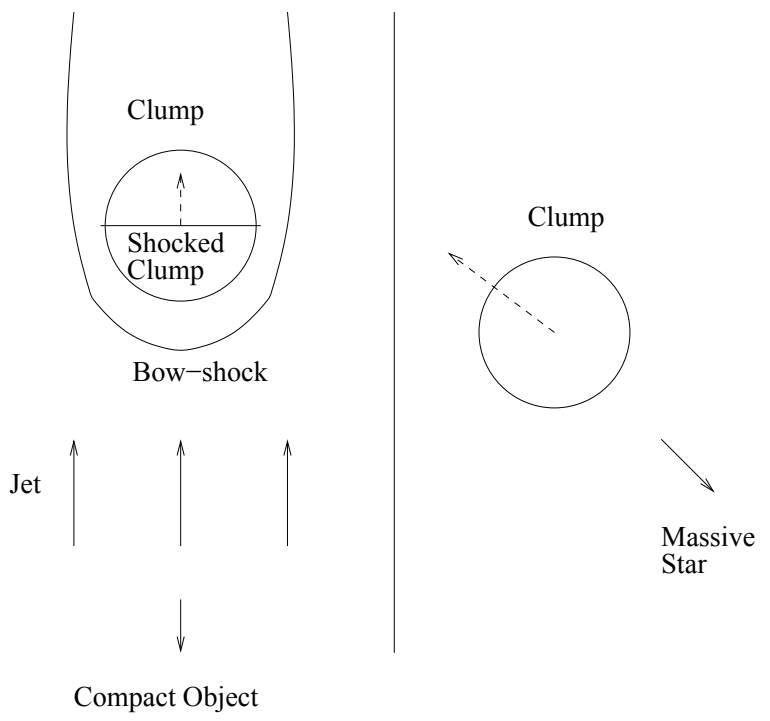

Figura 5.3: Esquema de la interacción jet-clump (Araudo et al. 2009).

por radiación térmica (de continuo y de líneas) resulta

$$
t_{\mathrm{ter}}=3 \times 10^{2} \frac{T_{\mathrm{c} 1}(x)^{1.6}}{n_{\mathrm{c} 1}(x)} \mathrm{s} \sim 10 \mathrm{~s} .
$$

Siendo $t_{\text {ter }}<t_{\text {cc }}$ podemos decir que el choque en el clump es radiativo. Este tiempo de enfriamiento corresponde a una distancia $x_{\mathrm{rad}} \sim t_{\text {ter }} v_{\mathrm{cc}} / 4 \sim 2 \times 10^{8} \mathrm{~cm}$, que es menor que $2 R_{\mathrm{c}}$. La velocidad $v_{\mathrm{cc}}$ de propagación del choque en el clump es calculada a través de la ecuación (2.47) y resulta $v_{\mathrm{cc}} \sim 7 \times 10^{7} \mathrm{~cm} \mathrm{~s}^{-1}$. A una distancia $>x_{\text {rad }}$ la temperatura del material chocado del clump es muy baja y la densidad crece hasta valores $\sim 10^{14} \mathrm{~cm}^{-3}$.

Aunque en este capítulo estamos interesados en la emisión de rayos gamma producida por la interacción jet-clump, hemos estimado por completitud la radiación libre-libre generada por el material chocado y calentado del clump. Considerando $n_{\mathrm{c} 1}(x)$ y $T_{\mathrm{c} 1}(x)$ estimamos la luminosidad por emisión libre-libre integrando la emisividad a lo largo del clump chocado (Lang 1999). Las luminosidades bolométricas obtenidas son $L_{\text {ter }} \sim 5 \times 10^{30}$ y $5 \times 10^{32} \mathrm{erg} \mathrm{s}^{-1}$ para $R_{\mathrm{c}}=10^{10}$ y $10^{11} \mathrm{~cm}$, respectivamente, con un máximo alrededor de los rayos $\mathrm{X}$ blandos $(\sim 1 \mathrm{keV})$. La luminosidad específica se muestra juntamente con la emisión no térmica en la Figura 5.8 .

Contrariamente al choque en el clump, el bow shock es adiabático y rápido. Por esta razón es un lugar propicio para la aceleración de partículas hasta energías relativistas, como veremos a continuación. 

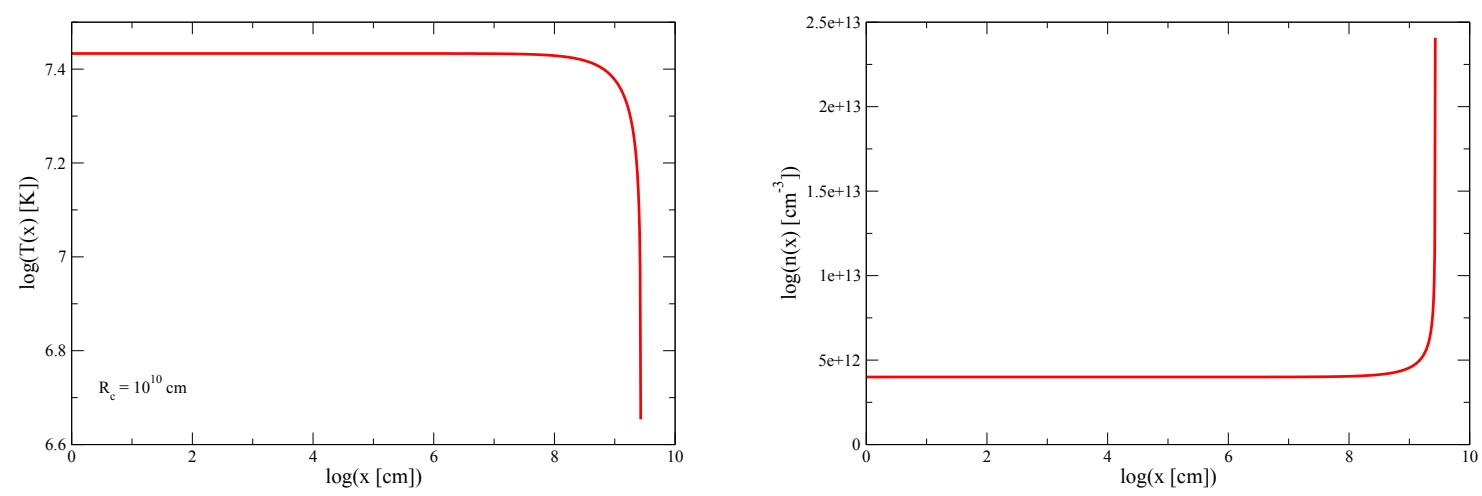

Figura 5.4: Variación de la temperatura (izquierda) y la densidad (derecha) en la región chocada del clump en función de la distancia $x$ al choque.

\subsection{Población de partículas relativistas}

En lo que sigue consideramos que las partículas relativistas son aceleradas en el bow shock únicamente. Los electrones y protones acelerados en este choque son inyectados siguiendo una distribución

$$
Q_{e_{1}, p}=K_{e_{1}, p} E_{e_{1}, p}^{-2} \exp \left(-\frac{E_{e_{1}, p}}{E_{e_{1}, p}^{\max }}\right) .
$$

Determinamos la constante de normalización $K_{e_{1}, p}$ suponiendo que el $25 \%$ de la luminosidad del jet inyectada en el bow shock, $L_{\mathrm{bs}} \sim\left(\sigma_{\mathrm{c}} / \sigma_{\mathrm{j}}\right) L_{\mathrm{j}}$, donde $\sigma_{\mathrm{c}}=\pi R_{\mathrm{c}}^{2}$ es la sección efectiva 2 del clump, se convierte en potencia de inyección de las partículas relativistas. Así, fijando que $L_{e, p}=0.25 L_{\mathrm{bs}}$ hallamos

$$
K_{e_{1}, p}=0.25\left(\frac{R_{\mathrm{c}}}{R_{\mathrm{j}}}\right)^{2} \frac{L_{\mathrm{j}}}{\ln \left(E_{e_{1}, p}^{\max } / E_{e_{1}, p}^{\min }\right)} .
$$

\subsubsection{Aceleración de partículas y pérdidas radiativas}

Para calcular el tiempo de aceleración $t_{\mathrm{ac}}$, necesitamos conocer el valor del campo magnético $B_{\mathrm{bs}}$ en la región del bow shock. Consideramos dos valores para esta magnitud. En primer lugar, estimamos el $B_{\text {bs }}$ resultante de imponer que la densidad de energía magnética $u_{\mathrm{B}}=B_{\mathrm{bs}}^{2} /(8 \pi)$ es el $10 \%$ de la densidad de energía del material chocado del jet, $u_{\mathrm{j} 1}$, cuya expresión es la siguiente:

$$
u_{\mathrm{j} 1}=\frac{3}{2} P_{\text {cin }}=\frac{9}{8} n_{\mathrm{j}} m_{p} v_{\mathrm{j}}^{2},
$$

\footnotetext{
${ }^{2}$ Despreciamos la región donde el bow shock se hace muy oblicuo y nos focalizamos en aquella en la cual el choque es más fuerte, es decir, en el frente del clump.
} 
donde $P_{\text {cin }}=\rho v^{2}$ es la presión cinética de un medio con densidad $\rho$ que se mueve con velocidad $v$. Fijando

$$
\frac{B_{\mathrm{bs}}^{2}}{8 \pi}=0.1 u_{\mathrm{j} 1}
$$

obtenemos $B_{\mathrm{bs}} \sim 150 \mathrm{G}$, lo que nos da un tiempo de aceleración para choques perpendiculares $t_{\mathrm{ac}}^{\perp} \sim 10^{-2} E_{e_{1}, p} \mathrm{~s}$ (ver la ecuación (2.60)). Por otro lado, hemos adoptado un valor mucho más bajo, $B_{\mathrm{bs}} \sim 1 \mathrm{G}$, para chequear el impacto de considerar un campo magnético mucho menos intenso que $150 \mathrm{G}$. Con $B_{\mathrm{bs}} \sim 1 \mathrm{G}$, el tiempo de aceleración resulta $t_{\mathrm{ac}}^{\perp} \sim 0.2 E_{e_{1}, p} \mathrm{~s}$.

Además de acelerarse, las partículas pueden escapar de la región chocada del jet ya sea por pérdidas difusivas o convectivas. El tiempo de convección por los costados del clump puede estimarse de la siguiente manera:

$$
t_{\text {conv }} \sim \frac{R_{\mathrm{c}}}{v_{\text {bs }} / 4} \sim 4\left(\frac{R_{\mathrm{c}}}{10^{10} \mathrm{~cm}}\right) \mathrm{s} .
$$

Por otro lado, el tiempo de difusión desde el bow shock hasta el clump considerando régimen de Bohm resulta

$$
t_{\mathrm{dif}}=\frac{Z^{2}}{D_{\mathrm{B}}} \sim 0.2\left(\frac{R_{\mathrm{c}}}{10^{10} \mathrm{~cm}}\right)^{2}\left(\frac{B_{\mathrm{bs}}}{1 \mathrm{G}}\right) \frac{1}{E_{e_{1}, p}} \mathrm{~s} .
$$

Notamos entonces que las partículas más energéticas pueden difundir hasta el clump antes de ser arrastradas por el material chocado del jet. Tanto en el clump como en la región del bow shock, los electrones y protones relativistas pierden energía a través de los diferentes procesos radiativos que hemos descripto en el Capítulo 3 y cuyas fórmulas aplicaremos a continuación.

\section{Pérdidas leptónicas}

En el caso con $B_{\mathrm{bs}}=150 \mathrm{G}$, la radiación sincrotrón es el mecanismo más eficiente de enfriamiento de los electrones en la región chocada del jet, con una escala de tiempo

$$
t_{\mathrm{sin}} \sim \frac{1.8 \times 10^{-2}}{E_{e_{1}}} \mathrm{~s},
$$

mientras que si $B_{\mathrm{bs}}=1 \mathrm{G}, t_{\mathrm{sin}} \sim 4 \times 10^{2} / E_{e_{1}}$ s. Para este último valor de $B_{b s}$ el proceso radiativo dominante resulta ser la dispersión IC.

A la altura del jet a la cual estamos considerando que ocurre la interacción jet-clump, $z_{\text {int }}=1.5 \times 10^{12} \mathrm{~cm}$, la densidad de energía de los fotones provenientes de la estrella compañera del HMMQ es $u_{\mathrm{ph} \star} \sim 2.4 \times 10^{-2} \mathrm{erg} \mathrm{cm}^{-3}$ y la energía promedio de estos fotones es $E_{\mathrm{ph} \star} \sim 3 K_{\mathrm{B}} T_{\star} \sim 10 \mathrm{eV}$. Para $y \equiv E_{\mathrm{ph} \star} E_{e} /\left(m_{e} c^{2}\right)^{2}>1$, esto es, $E_{e}>2.5 \times 10^{10} \mathrm{eV}$, la interacción IC ocurre en el régimen de $\mathrm{KN}$. El tiempo de enfriamiento tanto en el régimen de Th como en el de KN está dado por la expresión (ver (3.23)):

$$
t_{\mathrm{IC}} \sim 0.4 \frac{(1+8.3 y)}{\ln (1+0.2 y)} \frac{\left(1+1.3 y^{2}\right)}{\left(1+0.5 y+1.3 y^{2}\right)} \mathrm{s} .
$$



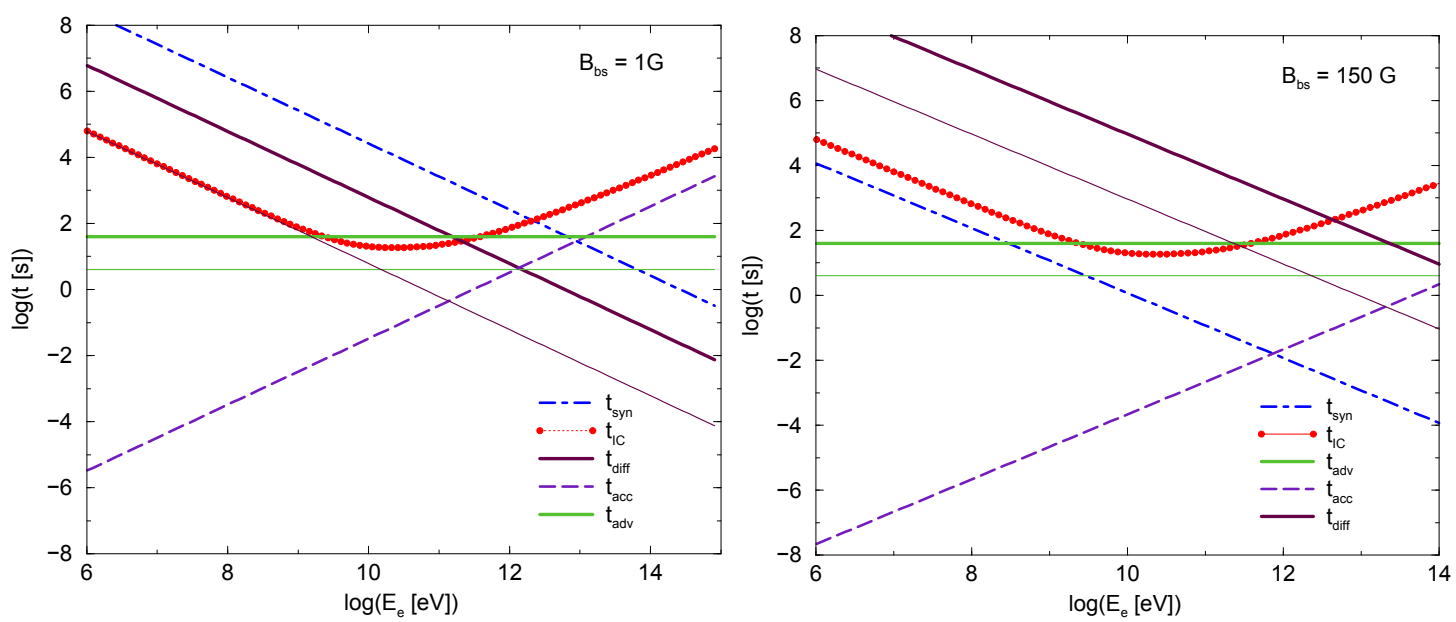

Figura 5.5: Tiempos de aceleración y de pérdidas radiativas (sincrotrón e IC) para electrones en la región del bow-shock para los casos $B_{\mathrm{bs}}=1 \mathrm{G}$ (izquierda) y $B_{\mathrm{bs}}=150 \mathrm{G}$ (derecha). Los tiempos de convección y difusión se muestran para $R_{\mathrm{c}}=10^{10}$ (línea fina) y $10^{11} \mathrm{~cm}$ (línea gruesa).

Por otro lado, las pérdidas por Bremsstrahlung relativista no son relevantes en ninguno de los dos casos (ni con $B_{\mathrm{bs}}=150 \mathrm{G}$ ni con $B_{\mathrm{bs}}=1 \mathrm{G}$ ), ya que la densidad del jet, $n_{\mathrm{j}}$, es muy baja a la altura $z_{\text {int }}$, con lo cual la densidad en la región del bow shock también resulta pequeña $\left(n_{\mathrm{bs}}=4 n_{\mathrm{j}} \sim 2 \times 10^{8} \mathrm{~cm}^{-3}\right)$. Con este valor de $n_{\mathrm{bs}}$ el tiempo de enfriamiento por Bremsstrahlung relativista resulta muy alto (ver (3.32) $)$

$$
t_{\mathrm{Brem}}=\frac{7 \times 10^{7}}{\ln \left(\frac{E_{e}}{m_{e} c^{2}}\right)+0.36} \mathrm{~s} .
$$

Teniendo en cuenta la ganancia por aceleración, las pérdidas radiativas y los tiempos de escape calculamos la energía máxima que pueden alcanzar los electrones acelerados en el bow shock. En el caso con $B_{\mathrm{bs}}=1 \mathrm{G}$, la energía máxima está determinada por las pérdidas difusivas, mientras que en el caso con $B_{\mathrm{bs}}=150 \mathrm{G}$ es la radiación sincrotrón el mecanismo de enfriamiento dominante a altas energías, como se muestra en la Figura 5.5. Como puede observarse en estos gráficos, la energía de quiebre $E_{\mathrm{q}}$ del espectro se obtiene igualando $t_{\text {conv }}=t_{\text {dif }}$, en el caso con $B_{\mathrm{bs}}=1 \mathrm{G}$ mientras que en el caso $B_{\mathrm{bs}}=150 \mathrm{G}$ el quiebre ocurre cuando $t_{\text {conv }}=t_{\sin }$. Los valores de las energías máximas y de quiebre obtenidos en cada caso se listan en la Tabla 5.3.

Teniendo en cuenta las pérdidas radiativas que sufren los electrones relativistas en la región del bow shock, calculamos la distribución espectral de energía de éstos, $N_{e_{1}}\left(E_{e_{1}}\right)$, a través de la ecuación (2.62) y teniendo en cuenta la constante de normalización del espectro de inyección dada por la ecuación (5.8). Consideramos un tiempo de duración de la interacción $\tau_{\text {vida }}>t_{\mathrm{cc}}$ y obtenemos un espectro en estado estacionario ya que los tiempos 
Tabla 5.3: Energías máximas alcanzadas por las partículas aceleradas en el bow shock. Los valores listados han sido calculados teniendo en cuenta los diferentes valores de $R_{\mathrm{c}}$ y $B_{\mathrm{bs}}$ considerados en este capítulo.

\begin{tabular}{c|cccc}
\hline \hline$R_{\mathrm{c}}[\mathrm{cm}]$ & $10^{10}$ & $10^{10}$ & $10^{11}$ & $10^{11}$ \\
$B_{\mathrm{bs}}[\mathrm{G}]$ & 1 & 150 & 1 & 150 \\
\hline$E_{e_{1}}^{\max }[\mathrm{eV}]$ & $1.5 \times 10^{11}$ & $8 \times 10^{11}$ & $1.5 \times 10^{12}$ & $8 \times 10^{11}$ \\
$E_{p}^{\max }[\mathrm{eV}]$ & $6 \times 10^{11}$ & $9 \times 10^{13}$ & $6 \times 10^{12}$ & $9 \times 10^{14}$ \\
$E_{\mathrm{q}}[\mathrm{eV}]$ & $3.1 \times 10^{10}$ & $2.8 \times 10^{9}$ & $3.1 \times 10^{11}$ & $2.8 \times 10^{8}$ \\
\hline
\end{tabular}
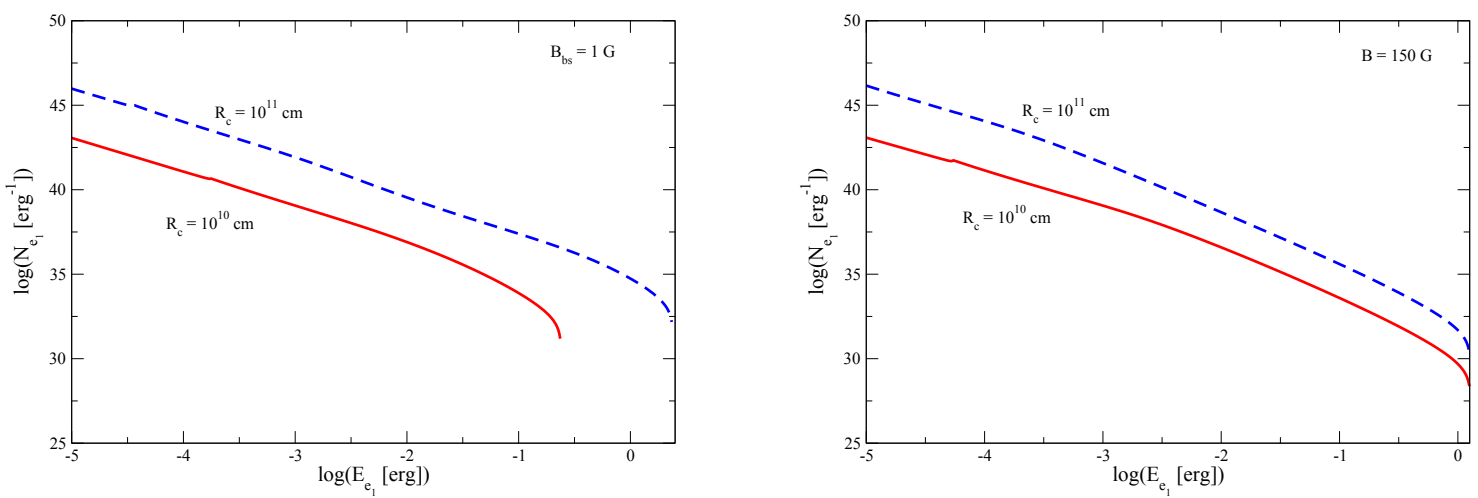

Figura 5.6: Distribución de energía de los electrones acelerados en el bow shock para los casos $B_{\mathrm{bs}}=1$ (izquierda) y $150 \mathrm{G}$ (derecha).

de pérdidas radiativas (sincrotrón e IC) y de escape son menores que $t_{\mathrm{cc}}$. Los resultados obtenidos se muestran en la Figura 5.6.

Finalmente, los electrones más energéticos $\left(E_{e_{1}}>0.3 E_{e_{1}}^{\max }\right)$ se podrían difundir hasta el clump. Esto ocurre si $t_{\text {dif }}<t_{\text {conv }}$ y $t_{\text {dif }}<t_{\text {rad }}$, donde la distancia que deben difundirse es el grosor del bow shock. Si los electrones llegan al clump, probablemente radíen allí toda su energía.

\section{Pérdidas hadrónicas}

De la misma manera que el Bremsstrahlung relativista es un canal de enfriamiento muy lento para los electrones en la región chocada del jet, el $p p$, con un ritmo de enfriamiento similar al Bremsstrahlung relativista, es también un proceso poco eficiente para los protones. En la región del bow shock $t_{p p}$ resulta $\sim 10^{7} \mathrm{~s}$, ya que la densidad del jet chocado es $n_{\mathrm{bs}} \sim 2 \times 10^{8} \mathrm{~cm}^{-3}$. La energía máxima de los protones relativistas acelerados en el bow 
shock queda determinada por el tiempo de difusión, dando

$$
E_{p}^{\max }=9 \times 10^{11}\left(\frac{R_{\mathrm{c}}}{10^{10} \mathrm{~cm}}\right)\left(\frac{B_{\mathrm{bs}}}{1 \mathrm{G}}\right) \mathrm{eV} .
$$

Los protones con energía $E_{p}>0.025 E_{p}^{\max }$ pueden difundir hasta el clump, ya que para estas energías $t_{\text {dif }}<t_{\text {conv }}$ en la dirección perpendicular al choque. Una vez allí, estos protones pueden interactuar con el material chocado del clump y emitir rayos gamma en un tiempo $t_{p p} \sim 500 \mathrm{~s}$. Para que los protones puedan estar confinados en el clump, el campo magnético de éste debe ser $B_{\mathrm{c}}>7.5 \times 10^{-3} B_{\mathrm{bs}}$, es decir, $B_{\mathrm{c}}>7.5 \times 10^{-3}$ ó $1.125 \mathrm{G}$, para el caso con $B_{\mathrm{bs}}=1$ y $150 \mathrm{G}$, respectivamente. Luego, los protones menos energéticos de aquéllos que lleguen al clump podrán ser confinados para valores de $B_{\mathrm{c}}$ razonables. Pero este límite de confinamiento es muy laxo, y en realidad el campo $B_{\mathrm{c}}$ necesario para confinar a los protones podría ser mucho mayor.

Aquí consideraremos el caso más conservativo para calcular la distribución de energía de los protones en el clump. Es decir, teniendo en cuenta que éstos permanecen en el clump sólo el tiempo que tardan en cruzarlo a una velocidad cercana a $c$, esto es, $t_{\text {cruce }} \sim R_{\mathrm{c}} / c \sim 0.3$ ó $3 \mathrm{~s}$ si $R_{\mathrm{c}}=10^{10}$ ó $10^{11} \mathrm{~cm}$, respectivamente. Siendo $t_{\text {cruce }}<t_{p p}$, la distribución de energía de los protones en el clump resulta

$$
N_{p}\left(E_{p}\right)=\frac{R_{\mathrm{c}}}{c} Q_{p}\left(E_{p}\right)
$$

donde $0.025 E_{p}^{\max }<E_{p}<E_{p}^{\max }$.

\subsection{Distribuciónes espectrales de energía}

\subsubsection{Emisión asociada al bow shock}

Como mostramos en la sección de pérdidas leptónicas, los mecanismos más eficientes de pérdidas radiativas son el sincrotrón y la dispersión IC. Sin embargo, debido a que la densidad de energía de la emisión sincrotrón es menor que la correspondiente al campo magnético $\left(u_{\mathrm{B}}\right)$ o a campos de radiación externos, la emisión por SSC no será importante y por lo tanto no la tenemos en cuenta en nuestros cálculos. Sólo consideramos los procesos sincrotrón e IC externo.

Calculamos $E_{\mathrm{ph}} L_{\mathrm{sin}}\left(E_{\mathrm{ph}}\right)$ y $E_{\mathrm{ph}} L_{\mathrm{IC}}\left(E_{\mathrm{ph}}\right)$ para los diferentes valores de $B_{\mathrm{bs}}$ y $R_{\mathrm{c}}$ considerados en este capítulo y los resultados se muestran en la Figura 5.7 Como puede verse en los gráficos, la componente debida a la radiación sincrotrón es más luminosa que la correspondiente al IC en los casos con $B_{\mathrm{bs}}=150 \mathrm{G}$, alcanzando luminosidades bolométricas $L_{\mathrm{sin}} \sim 10^{33}$ y $2 \times 10^{35} \mathrm{erg} \mathrm{s}^{-1}$ para $R_{\mathrm{c}}=10^{10}$ y $10^{11} \mathrm{~cm}$, respectivamente. Por el contrario, para $B_{\mathrm{bs}}=1 \mathrm{G}$, el proceso radiativo dominante es la interacción IC, con luminosidades bolométricas $L_{\mathrm{IC}} \sim 2 \times 10^{32}$ y $10^{35} \mathrm{erg} \mathrm{s}^{-1}$ para $R_{\mathrm{c}}=10^{10}$ y $10^{11} \mathrm{~cm}$, respectivamente. Las energías máximas alcanzadas por los fotones emitidos son $E_{\mathrm{ph}}^{\max } \sim 1 \mathrm{TeV}$. Notamos que tanto el espectro debido a la radiación sincrotrón como a la dispersión IC en el caso con 

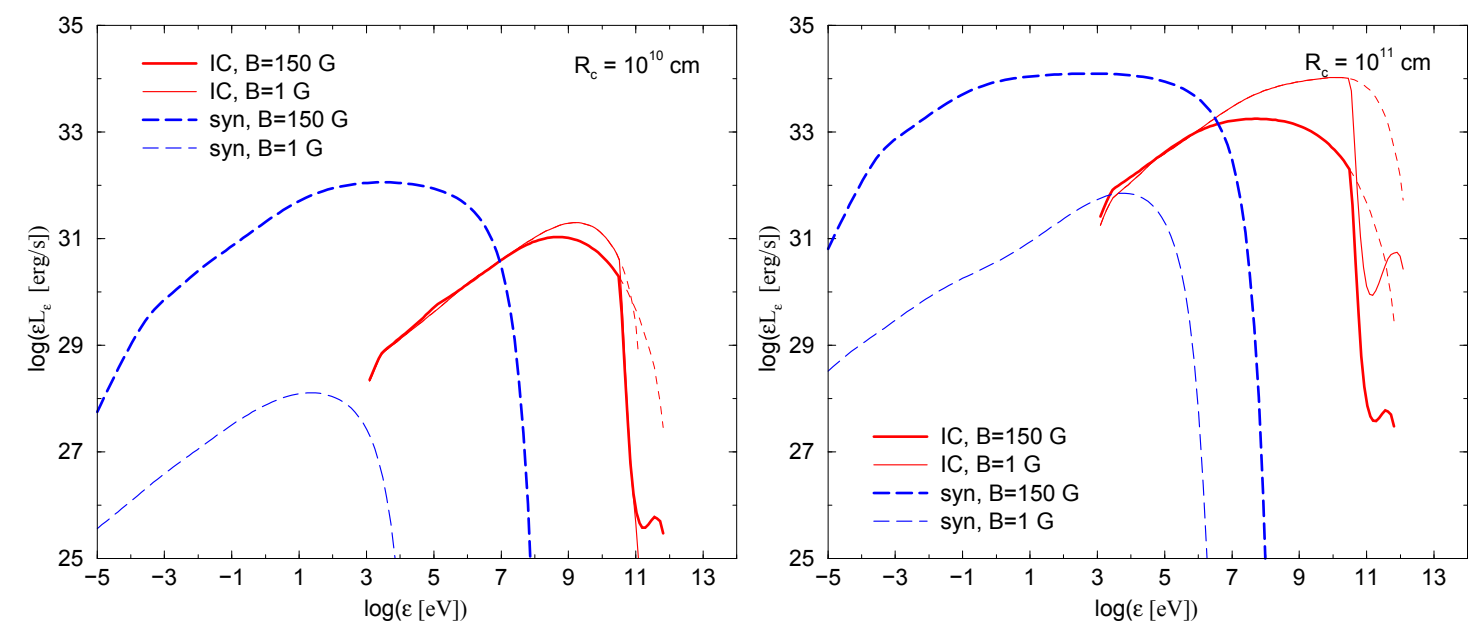

Figura 5.7: Radiación sincrotrón (línea segmentada azul) y IC (línea roja) producida en la región del bow-shock, para $R_{\mathrm{c}}=10^{10}$ (izquierda) y $R_{\mathrm{c}}=10^{10} \mathrm{~cm}$ (derecha), con $B_{\mathrm{bs}}=1$ (línea fina) y $150 \mathrm{G}$ (línea gruesa). Los efectos de la absorción gamma-gamma son mostrados (línea gruesa), juntamente con el espectro no absorbido (línea punteada fina).

$B_{\mathrm{bs}}=150 \mathrm{G}$ se quiebra de manera clara por efecto de las pérdidas por convección de las partículas de la región del bow shock. Además, el efecto de las pérdidas IC en el régimen de $\mathrm{KN}$ endurecen el espectro de los electrones en el caso con $R_{\mathrm{c}}=10^{11} \mathrm{~cm}$ y $B_{\mathrm{bs}}=1 \mathrm{G}$, lo cual se ve reflejado en el espectro de los fotones producidos por los mecanismos sincrotrón e IC. Sin embargo, notamos que los electrones relativistas aunque son arrastrados de la región del bow shock antes de que emitan significativamente en frecuencias radio, también pueden emitir en esta banda de energías en otras regiones del jet3.

En los cálculos de las SEDs hemos tenido en cuenta la absorción (gamma-gamma) por creación de pares $e^{ \pm}$en el campo de los fotones producidos por la estrella. Debido a que no nos focalizamos en la geometría del sistema HMMQ/observador, hemos supuesto que el campo de fotones semilla es isotrópico (también hemos despreciado los efectos angulares en el cálculo de la interacción IC). Como puede verse en la Figura 5.7, la absorción gammagamma reduce los niveles de emisión varios órdenes de magnitud a energías de cientos de $\mathrm{GeV}$. Solo en algunos casos, con geometrías específicas en la interacción gamma-gamma, la atenuación puede ser despreciable (Khangulyan et al. 2008).

\subsubsection{Emisión asociada al clump}

Las partículas más energéticas aceleradas en el bow shock pueden difundir hasta el clump y radiar allí. En el caso de los protones, aquellos con $E_{p}>0.025 E_{p}^{\max }$ pueden llegar

\footnotetext{
${ }^{3}$ El cálculo de esta emisión escapa a los intereses de nuestro estudio. Por esta razón, no hemos tenido en cuenta los efectos de la autoabsorción sincrotrón en el espectro en frecuencias radio y no haremos predicciones en este rango de energías.
} 
al clump. Por otro lado, en el caso $B_{\mathrm{bs}}=1 \mathrm{G}$, los electrones con $E_{e_{1}}>0.3 E_{e_{1}}^{\max }$ son los que pueden difundir una distancia $Z$ y perder toda su energía en el clump.

A la emisión $p p$ de los protones que difunden hasta el clump la calculamos usando las ecuaciones (3.39) y (3.41), y considerando que $N_{p}\left(E_{p}\right)$ está determinada por la fórmula (5.17). La emisión por $p p$ alcanza una luminosidad $\sim 10^{32} \mathrm{erg} \mathrm{s}^{-1}$ a una energía $E_{\mathrm{ph}} \sim 50 \mathrm{GeV}$, como se muestra en la Figura 5.8, junto con las contribuciones leptónicas de la región del bow shock. Además de rayos gamma, en las interacciones $p p$ también se producen pares $e^{ \pm}$y neutrinos de muy altas energías, siendo la luminosidad de estos últimos $\sim L_{p p}$ (Aharonian et al. 2006; Reynoso \& Romero 2009). Los pares $e^{ \pm}$radían casi toda su energía en el clump por los procesos sincrotrón, IC y Bremsstrahlung relativista, pero la contribución de los electrones primarios tanto en la región del bow shock como en el clump es mayor que la de los pares (Bosch-Ramon et al 2005; Orellana et al. 2007).

Para estimar la radiación producida en el clump por los electrones primarios acelerados en el bow shock y que difunden hasta allí, suponemos dos valores para el campo magnético del clump: $B_{\mathrm{c}}=1$ y $100 \mathrm{G}$, siendo el último similar al valor de equipartición entre la densidad de energía magnética y térmica. A las luminosidades específicas de cada proceso (sincrotrón, IC y Bremsstrahlung relativista) las calculamos con las fórmulas dadas en el Capítulo 3, Como se muestra en la Figura 5.9, la componente sincrotrón domina a la debida a las interacciones IC en el caso con $B_{\mathrm{c}}=100 \mathrm{G}$, alcanzando una luminosidad $\sim 10^{34} \mathrm{erg} \mathrm{s}^{-1}$ $\left(R_{\mathrm{c}}=10^{11} \mathrm{~cm}\right)$ a $E_{\mathrm{ph}} \gtrsim 1 \mathrm{MeV}$. Por otro lado, para $B_{\mathrm{c}}=1 \mathrm{G}$ la componente IC absorbida (por gamma-gamma) alcanza una luminosidad similar a la sincrotrónica y es $\sim 10^{33} \mathrm{erg} \mathrm{s}^{-1}$. La emisión por Bremsstrahlung relativista es despreciable en ambos casos. Las SEDs para el caso $R_{\mathrm{c}}=10^{10} \mathrm{~cm}$, y para ambos valores de $B_{\mathrm{c}}$, son morfológicamente similares a las correspondientes mostradas en la Figura [5.9. pero las luminosidades alcanzadas son aproximadamente dos órdenes de magnitud menores, ya que éstas son $\propto \sigma_{c}$.

Notamos finalmente que la emisión no térmica del clump es similar, aunque levemente menos intensa, a la producida en la región del bow shock, siendo el espectro más duro. Esto es debido a que las partículas inyectadas en el clump tienen energías $>0.3 E_{e_{1}}^{\max }$. Por claridad, no graficamos la emisión leptónica del clump jutamente con las demás componentes (leptónicas de la región del bow shock y hadrónica del clump).

\subsection{Interacciones simultáneas}

Hasta aquí solo hemos considerado la interacción de un clump con el jet a la altura $z_{\text {int }}=a_{\mathrm{mq}} / 2$. Sin embargo, muchos clumps pueden estar simultáneamente interactuando con el jet a diferentes $z_{\mathrm{j}}$ (Owocki et al. 2009).

La altura mínima $z_{\text {int }}^{\text {min }}$, a la cual los clumps pueden penetrar completamente dentro del jet sin ser destruidos en el proceso, es aquella para la cual $t_{\mathrm{c}}<t_{\mathrm{cc}}$. Esto determina un $z_{\text {int }}^{\text {min }} \sim 4 \times 10^{11} \mathrm{~cm}$. De esta manera, el modelo presentado en este capítulo para la interacción jet-clump es válido sólo para $z_{\text {int }}>4 \times 10^{11} \mathrm{~cm}\left(\mathrm{y}\right.$ para $R_{\mathrm{c}}<R_{\mathrm{j}}$ ). Considerando que el jet presenta una geometría cónica, calculamos el número de clumps $N_{\mathrm{c}}$ que pueden estar simultáneamente dentro del jet. Para esto integramos desde $z=z_{\text {int }}^{\text {min }}$ hasta $z=a_{\mathrm{mq}}$ 

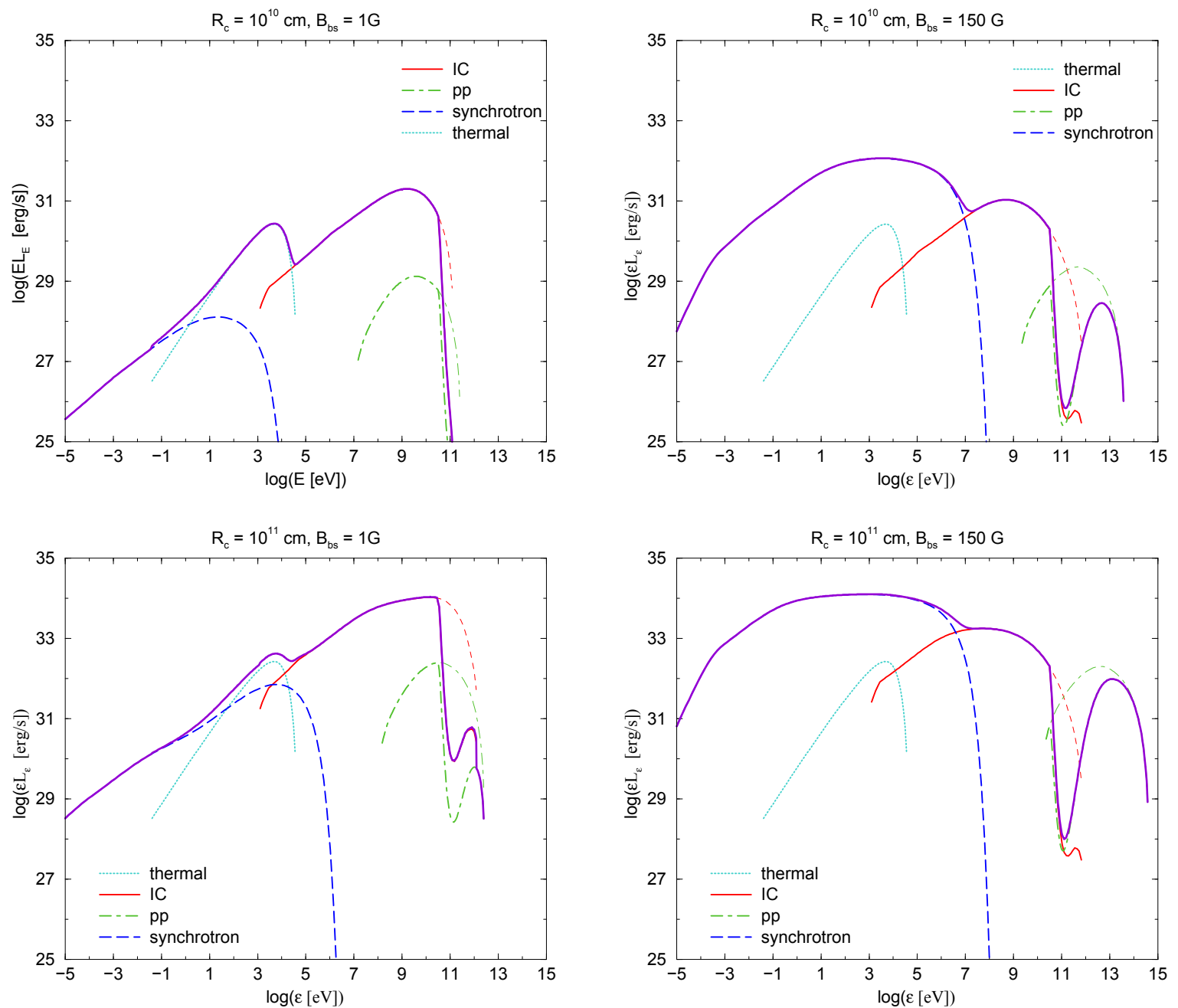

Figura 5.8: SEDs de la emisión sincrotrón, IC, $p p$ y térmica para diferentes valores de $B_{\mathrm{bs}}$ y $R_{\mathrm{c}}$; las curvas de la componente absorbida y no absorbida (líneas finas) de la radiación IC y $p p$ son graficadas. 


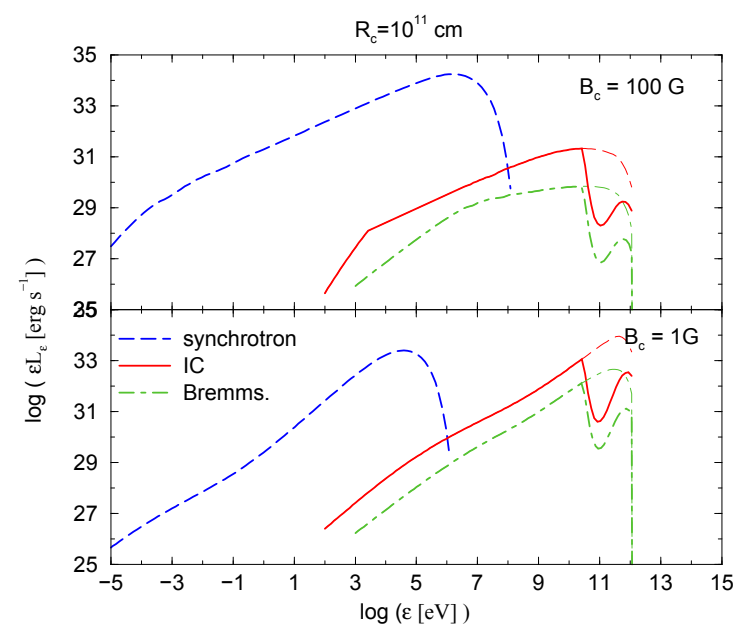

Figura 5.9: Emisión no térmica del clump, para el caso con $R_{\mathrm{c}}=10^{11} \mathrm{~cm}$ y para los dos valores de $B_{\mathrm{c}}$ asumidos. Las curvas correspondientes a la emisión por IC y Bremsstrahlung relativista se muestran absorbidas (líneas gruesas) y no absorbidas (líneas finas).

y asumimos que el factor de llenado de clumps en el jet es el mismo que en el viento, $f=0.005$. Así hallamos $N_{\mathrm{c}} \sim 350$ y 0.5 para $R_{\mathrm{c}}=10^{10}$ y $10^{11} \mathrm{~cm}$, respectivamente. Como consecuencia de estos resultados, los flares producidos por la interacción de un clump con el jet serán un fenómeno esporádico para $N_{\mathrm{c}}$ bajo $\left(R_{\mathrm{c}}=10^{11} \mathrm{~cm}\right)$, o será una modulación de la emisión continua de la fuente, para $N_{\mathrm{c}}$ alto $\left(R_{\mathrm{c}}=10^{10} \mathrm{~cm}\right)$ (ver Owocki et al. 2009). En el último caso, la SED resultante será morfológicamente similar a las mostradas en las secciones previas, pero multiplicada por $N_{\mathrm{c}}$, como se muestra en la Figura 5.10. Sin embargo, notamos que por un lado el jet puede verse dinámicamente afectado si muchos clumps están simultáneamente dentro de él. Por el otro, a diferentes alturas de interacción, la luminosidad no térmica disponible para radiar es distinta (disminuyendo con $z_{\mathrm{j}}$ ) y por lo tanto la SED resultante no es un simple escaleo con $N_{\mathrm{c}}$ como el mostrado en la Figura 5.10,

\subsection{Discusión}

En este capítulo exploramos los procesos físicos más relevantes y la naturaleza de la radiación producida por la interacción de un clump del viento de la estrella primaria con el jet de un HMMQ. La interacción jet-clump produce dos choques: uno en el jet y otro en el clump. El primero alcanza rápidamente el estado estacionario formando un bow shock en el jet mientras que el segundo se propaga a través del clump empujado por la presión del medio chocado, que se equilibra con la presión del material del jet chocado. El bow shock es adiabático y rápido, y partículas cargadas pueden acelerarse hasta energías muy altas allí mediante el mecanismo de Fermi. En la región del bow shock los electrones relativistas se enfrían eficientemente por radiación sincrotrón e IC. Por otro lado, el choque en el clump 

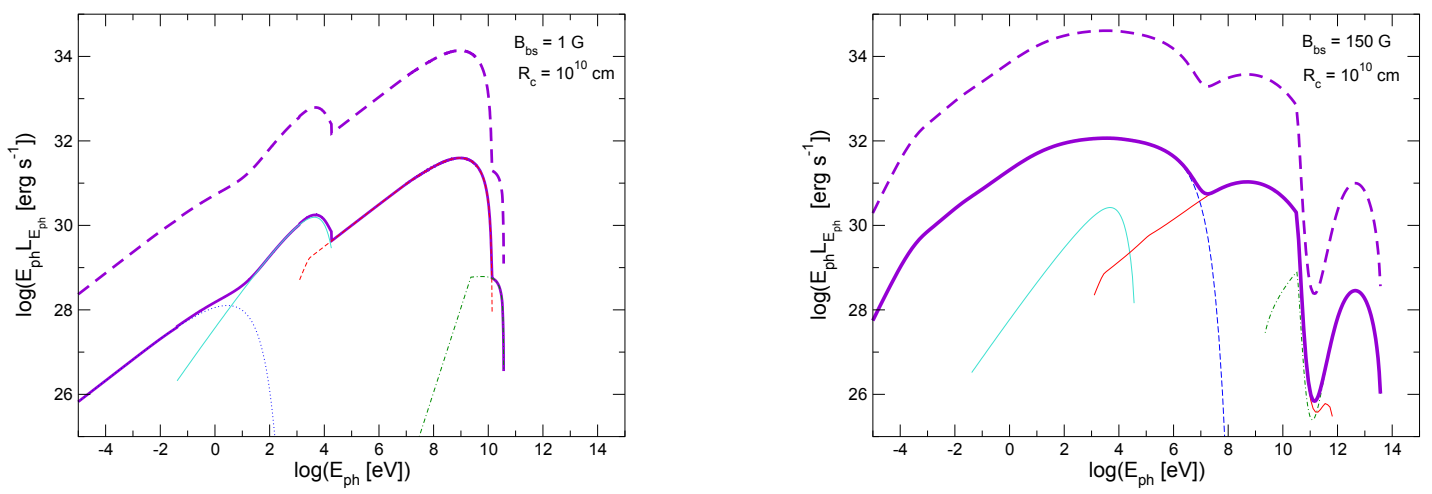

Figura 5.10: Distribuciones espectrales de energía de la emisión sincrotrón, IC, $p p$ y térmica para el caso con clumps de $R_{\mathrm{c}}=10^{10} \mathrm{~cm} \mathrm{y} B_{\mathrm{bs}}=1$ (derecha) y $150 \mathrm{G}$ (derecha). Hemos tenido en cuenta la contribución de todos los clumps que simultáneamente se encuentran dentro del jet.

es lento y radiativo, no siendo eficiente para acelerar partículas. Sin embargo, la emisión térmica del material chocado del clump podría ser significativa, como así también podrían serlo las interacciones $p p$ entre los protones relativistas acelerados en el bow shock que difunden hasta el clump y el material chocado de éste. Si los electrones acelerados en el bow shock llegan hasta el clump, éstos pueden a su vez radiar en el clump eficientemente a través de los procesos sincrotrón e IC. Las SEDs de las componentes radiativas mencionadas anteriormente han sido calculadas en el contexto de un HMMQ con parámetros similares a los del sistema Cygnus X-1 y los resultados se muestran en las Figuras 5.8 y 5.9.

En rayos X, la emisión es producida por radiación sincrotrón (en el bow shock y en el clump) y térmica (en el clump). En los casos con $B_{\mathrm{bs}} \sim 1 \mathrm{G}$, la emisión térmica alcanza luminosidades $L_{\text {ter }} \sim 10^{32} \mathrm{erg} \mathrm{s}^{-1}$ siendo así mayor que la sincrotrón emitida en la región del bow-shock, pero no mayor que la emitida en el clump si $R_{\mathrm{c}}=10^{11} \mathrm{~cm}$. La emisión sincrotrón de la región del bow-shock es dominante en rayos X para $B_{\mathrm{bs}}=150 \mathrm{G}$, alcanzando $L_{\text {sin }} \sim 10^{35} \mathrm{erg} \mathrm{s}^{-1}$. En una fuente como Cygnus X-1, estos niveles de emisión en rayos X serían superados por la radiación del disco de acreción. Sin embargo, en el caso de fuentes poco luminosas en rayos X, como LS 5039 y LS I +61 303 (Bosch-Ramon et al. 2007, Paredes et al. 2007), los rayos X producidos via el proceso sincrotrón durante la interacción jetclump deberían ser detectables, y aún la componente térmica debería ser detectable bajo determinadas condiciones (clumps grandes con densidades relativamente bajas).

Las dispersiones IC en la región del bow-shock y en el clump producen rayos gamma hasta VHE, dominando la SED en los casos con campos magnéticos relativamente bajos $\left(B_{\mathrm{bs}}=1 \mathrm{G}\right)$. En nuestros cálculos, la luminosidad más alta alcanzada es $L_{\mathrm{IC}} \sim 10^{35} \mathrm{erg} \mathrm{s}^{-1}$ para $R_{\mathrm{c}}=10^{11} \mathrm{~cm}$, aunque la absorción gamma-gamma puede reducir sustancialmente la emisión por encima de los $100 \mathrm{GeV}$ (Romero et al 2010). Las interacciones pp en el clump 
pueden también producir rayos gamma a energías tan altas como $\sim 10^{14} \mathrm{eV}\left(B_{\mathrm{bs}}=150 \mathrm{G}\right)$. La luminosidad máxima obtenida por $p p$ es sin embargo modesta, $L_{p p} \sim 10^{32} \mathrm{erg} \mathrm{s}^{-1}$ para $R_{\mathrm{c}}=10^{11} \mathrm{~cm}$, aunque clumps más densos y/o más grandes, y jets más poderosos producirían cantidades detectables de fotones fuera del rango (0.1-10 TeV) donde la absorción gamma-gamma es importante. Recordamos que una geometría específica del sistema binario/observador mas un emisor de altas energías lejos del objeto compacto puede dar una atenuación de los rayos gamma mucho menor (e.g. Khangulyan et al. 2008).

Como consecuencia de las características de la interacción, la emisión esperada es transitoria (tipo flare). La duración de esta emisión está relacionada con la permanencia del clump dentro del jet, lo cual depende fuertemente de las inestabilidades de RT y KH, las cuales pueden destruir el clump. Debido a que el clump puede ser acelerado dentro del jet, el tiempo de vida del primero es de algunas veces el tiempo característico $t_{\mathrm{cc}}$. Si el clump no ha sido destruido, éste puede eventualmente salir del jet luego de haber sido chocado y calentado. Dadas las escalas de tiempo dinámicas de la interacción, el evento tendrá una duración de entre unos pocos minutos y algunas horas.

Los flares producidos en las interacciones jet-clump pueden tener asociados componentes espectrales a bajas (radiación sincrotrón y emisión térmica) y altas energías (interacciones IC y $p p$ ), las cuales no tienen que estar correlacionadas con la actividad de acreción del disco. El nivel total de emisión, la importancia relativa de las diferentes componentes de la SED y la duración de los flares pueden proveer de información sobre la potencia del jet, como así también del tamaño y la densidad de los clumps y el valor del campo magnético en la región donde ocurre la interacción (Romero et al. 2007). Por lo tanto, además de las propiedades del jet mismo, las características de los clumps pueden ser testeadas a través de observaciones en HE y VHE (y probablemente también en frecuencias radio) de los flares producidos en HMMQs, abriendo una nueva ventana del espectro electromagnético para estudiar los vientos de las estrellas de gran masa.

Dependiendo del factor de llenado $f$ del viento (o de la densidad de los clumps) y de $R_{\mathrm{c}}$, el número de clumps $-N_{\mathrm{c}^{-}}$que simultáneamente pueden estar dentro del jet puede ser bajo $(<1)$ o alto, esto es, un clump eventualmente o muchos simultáneamente. Luego, los flares producidos por las interacciones de clumps con los jets en HMMQs pueden ser un fenómeno esporádico ( $N_{\mathrm{c}}$ pequeño) o pueden aparecer como una modulación estacionaria en el espectro (flickering) ( $N_{\mathrm{c}}$ alto). Sin embargo, notamos que el jet puede verse sustancialmente afectado si muchos clumps están simultáneamente dentro de él. Asumiendo que la ruptura del jet tiene lugar para $\sigma_{\mathrm{j}}<N_{\mathrm{c}} \times \sigma_{\mathrm{c}}$, para los parámetros del viento y del jet adoptados en este capítulo, el jet podría ser destruido si $R_{\mathrm{c}}<10^{10} \mathrm{~cm}$ con el valor de $f$ adoptado. Sin embargo, cálculos más detallados de la dínamica de la interacción jet-clump son requeridos para clarificar este hecho. En el próximo capítulo, haremos un estudio un poco más minucioso de la interacción simultánea de muchos obtáculos con jets, pero en el contexto de los AGNs. 


\section{Capítulo 6}

\section{Núcleos de galaxias activas}

\subsection{Introducción}

Los núcleos de las galaxias albergan agujeros negros supermasivos (SMBHs, por Super Massive Black Holes) los cuales abarcan un amplio rango de masas: $10^{6} \lesssim M_{\text {smbh }} \lesssim$ $10^{10} M_{\odot}$. El proceso de formación de estos SMBHs es a través de la captura de material del medio circundante, ya sean nubes de gas, estrellas o cúmulos de estrellas (Rees 1984). Dependiendo del momento angular del SMBH y de la materia, la acreción será esférica o no. Estos SMBHs pueden estar activos o no, dependiendo de la tasa de acreción de materia. Podemos decir que la actividad es una etapa en la vida de las galaxias que depende fuertemente de la cantidad de materia que haya en las cercanías del SMBH. Si la acreción es suficiente como para que se forme un disco y consecuentemente los jets, entonces se dice que tenemos una galaxia activa.

Actualmente, el modelo más aceptado para describir a los AGN es el que se conoce como modelo estándar. Este considera que las galaxias activas son un sistema compuesto por un SMBH rotante como objeto central, circundado por un disco de acreción el cual a su vez está rodeado por un toro de gas y polvo. Además el sistema se compone de dos jets paralelos al eje de rotación del SMBH y que se propagan en sentidos opuestos. En la Figura 6.1 se muestran las principales componentes de un AGN. De acuerdo al modelo estándar, la actividad de los AGN es un fenómeno intrínsecamente anisotrópico. Consecuentemente, la fenomenología observada dependerá del ángulo de inclinación entre la línea de la visual y algún eje de simetría de la fuente, como por ejemplo el eje de rotación del SMBH. Cuando este ángulo es cercano a $90^{\circ}$ estamos observando o bien una galaxia de líneas delgadas (NLRG, por Narrow Line Region Galaxy) o bien una galaxia Seyfert del tipo II; mientras que si dicho ángulo es prácticamente nulo, tenemos un blazar. En cualquier caso intermedio el objeto observado será un cuasar radio-silencioso, una galaxia de líneas anchas (BLRG, por Broad Line Region Galaxy) o una galaxia Seyfert del tipo I. Por otro lado, las radiogalaxias son AGN que presentan una fuerte emisión en frecuencias radio. Los jets de estas fuentes no están alineados con la línea de la visual, es decir, no son blazares, y se clasifican de acuerdo a la luminoisidad de los mismos. Las radiogalaxias 


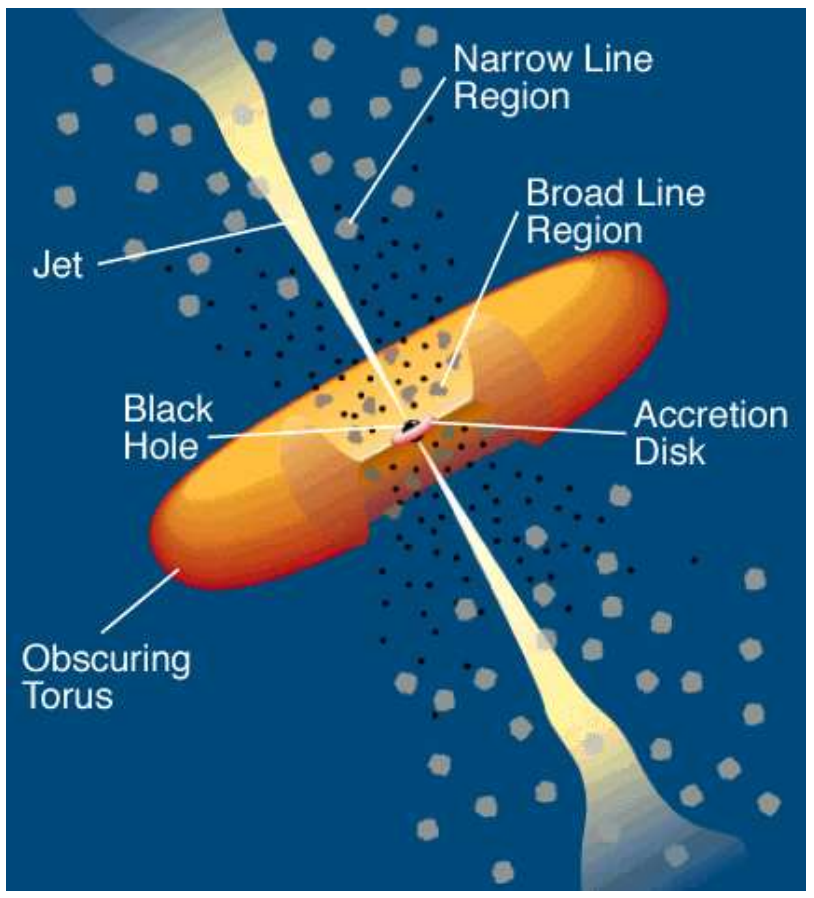

Figura 6.1: Modelo estándar de AGN. Se muestran las componentes más importantes de acuerdo a este modelo: el SMBH, el disco de acreción, el toro, los jets y las regiones donde se emiten las líneas anchas y delgadas (Urry \& Padovani 1995). 


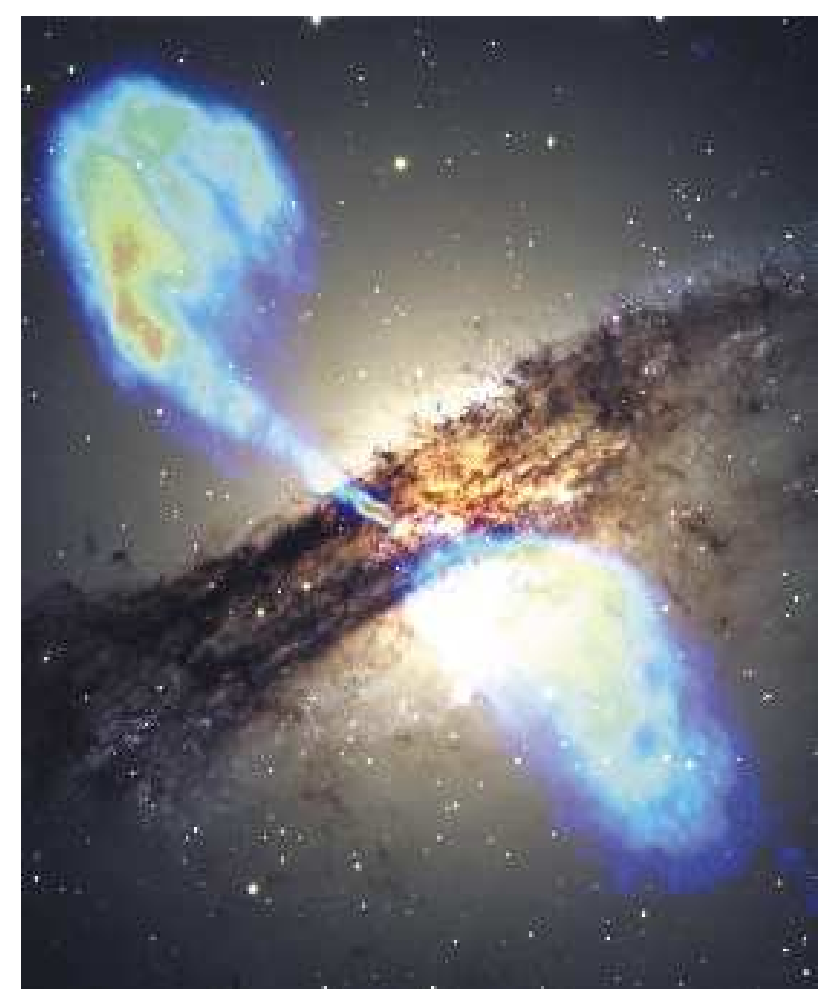

Figura 6.2: Imagen de la radiogalaxia Centaurus A. La imagen muestra esta galaxia activa en radio y en el óptico. La emisión a bajas frecuencias corresponde a los jets y a los lóbulos, y se piensa que es producida por el proceso sincrotrón. La emisión óptica corresponde a la radiación térmica del gas y del polvo que se encuentran tapando la región nuclear de la galaxia. (Crédito: NASA.)

Faranoff-Riley I (FR I) son menos luminosas que las FR II (Fanaroff \& Riley, 1974) por lo cual los jets de las primeras pueden propagarse una distancia mayor que los jets de las FR II antes de ser frenados por el medio externo.

Una de las características principales de los AGN es que la emisión continua abarca casi todo el espectro electromagnético, desde frecuencias radio hasta los rayos gamma. Esta radiación proviene básicamente del disco (térmica) y de los jets (no térmica). En la Figura 6.2 se muestra una imagen compuesta de la galaxia activa Centaurus A (Cen A) en radio y en óptico.

Además del espectro continuo, los AGN emiten líneas en óptico y en UV. El mecanismo más aceptado para explicar la emisión de estas líneas es que el medio cerca del SMBH no es homogéneo sino que tiene una estructura porosa en forma de nubes o estrellas. La región donde se forman las líneas de emisión más anchas (BLR, por Broad Line Region) está formada por nubes de material del disco o del medio circundante. Este material está confinado por el medio externo caliente, cuya temperatura es $\sim 10^{8} \mathrm{~K}$, (Krolik et al. 1981) o por campos magnéticos (Rees 1987). La materia que forma estas nubes puede ser ionizada 
por los fotones emitidos en el disco de acreción produciendo líneas de emisión. Luego estas líneas son ensanchadas debido a que las nubes se mueven en el pozo de potencial del SMBH con una velocidad $v_{\mathrm{n}}>1000 \mathrm{~km} \mathrm{~s}^{-1}$. Otro modelo para la producción de las líneas anchas detectadas en algunos AGN es aquel en el cual la BLR está compuesta por estrellas evolucionadas (gigantes rojas) cuyas cromósferas son fotoionizadas (Penston 1988).

La BLR rodea al agujero negro y por lo tanto la interacción de algunas de las nubes que la componen con la parte más interna de los jets es factible. En este capítulo estudiamos la interacción de nubes de la BLR con la base de los jets, realizando un tratamiento similar al desarrollado en el capítulo anterior para los HMMQ. Los choques producidos por la penetración de estas nubes en los jets pueden acelerar partículas hasta velocidades relativistas, las cuales luego pueden enfriarse por diferentes procesos no térmicos produciedo niveles detectables de emisión en rayos gamma. La detección de esta radiación nos proveería información valiosa sobre las condiciones ambientales en las cercanías de la base de los jets como así también de las propiedades de la BLR.

\subsection{Escenario}

Bajo ciertas relaciones entre la presión cinética del jet y la densidad y el tamaño de las nubes, la penetración de una nube en el jet es factible. Los detalles del proceso de penetración en si mismo son complejos, y en esta tesis no tratamos esto en detalle aunque supondremos que la penetración ocurre si se satisfacen ciertas condiciones. Un esquema de la interacción se muestra en la Figura 6.3.

A diferencia del tratamiento hecho en el capítulo anterior de la interacción jet-clump, donde consideramos un escenario similar a la fuente Cygnus X-1, en este capítulo no planteamos un escenario definido, asociado a un AGN específico, sino que hacemos un tratamiento más general de la interacción. Dejamos fijos a lo largo de todo el capítulo sólo aquellos parámetros estándares que, en principio, no varían sustancialmente de una galaxia a otra.

\section{Modelo para las nubes}

Supondremos en este estudio nubes esféricas, cuyo radio fijamos en $R_{\mathrm{n}}=10^{13} \mathrm{~cm}$ (Risaliti 2009), y con una densidad uniforme típica $n_{\mathrm{n}}=10^{10} \mathrm{~cm}^{-3}$. La velocidad de las nubes está fijada en $v_{\mathrm{n}}=10^{9} \mathrm{~cm} \mathrm{~s}^{-1}$ (Peterson 2006).

\section{Modelo para los jets}

Suponemos que los jets son hidrodinámicos y relativistas, con un factor de Lorentz $\Gamma_{\mathrm{j}}=10$, lo cual implica una velocidad $v_{\mathrm{j}} \sim c$. A su vez fijamos el ángulo de apertura $\phi \approx 6^{\circ}$, es decir, la relación entre el radio y la altura del jet resulta $R_{\mathrm{j}}=\tan (\phi) z_{\mathrm{j}} \sim 0.1 z_{\mathrm{j}}$. La densidad de los jets a una altura $z_{\mathrm{j}}$ y en el SR del laboratorio puede estimarse a través 
Tabla 6.1: Valores asumidos en este trabajo para las nubes de la BLR y para los jets.

\begin{tabular}{ll}
\hline \hline Descripción & Valor \\
\hline Tama no de las nubes & $R_{\mathrm{n}}=10^{13} \mathrm{~cm}$ \\
Densidad de las nubes & $n_{\mathrm{n}}=10^{10} \mathrm{~cm}^{-3}$ \\
Velocidad de las nubes & $v_{\mathrm{n}}=10^{9} \mathrm{~cm} \mathrm{~s}^{-1}$ \\
Temperatura de las nubes & $T_{\mathrm{n}}=2 \times 10^{4} \mathrm{~K}$ \\
Factor de Lorentz de los jets & $\Gamma_{\mathrm{j}}=10$ \\
Angulo de semi-apertura de los jets & $\phi \approx 6^{\circ}$ \\
\hline
\end{tabular}

de la relación (2.43), con la cual obtenemos

$$
n_{\mathrm{j}}=\frac{L_{\mathrm{j}}}{\left(\Gamma_{\mathrm{j}}-1\right) m_{p} c^{3} \sigma_{\mathrm{j}}} \approx 8 \times 10^{4}\left(\frac{L_{\mathrm{j}}}{10^{44} \mathrm{erg} \mathrm{s}^{-1}}\right)\left(\frac{\Gamma_{\mathrm{j}}-1}{9}\right)^{-1}\left(\frac{z_{\mathrm{j}}}{10^{16} \mathrm{~cm}}\right)^{-2} \mathrm{~cm}^{-3},
$$

donde $L_{\mathrm{j}}$ es la luminosidad cinética de los jets y $\sigma_{\mathrm{j}}=\pi R_{\mathrm{j}}^{2}$. En la Tabla 6.1 se listan los valores de los parámetros de las nubes y de los jets que quedarán fijos a lo largo de todo el capítulo.

\subsubsection{Interacción jet-nube}

El modelo de interacción de una nube de la BLR con uno de los jets del AGN es similar al descripto en el capítulo anterior pero en este escenario el jet es relativista.

Una de las condiciones que deben satisfacerse para que la nube pueda entrar entera en el jet es que la presión cinética de éste no la destruya en el proceso de penetración. Esto significa que el tiempo de penetración,

$$
t_{\mathrm{n}} \sim \frac{2 R_{\mathrm{n}}}{v_{\mathrm{n}}}=2 \times 10^{4}\left(\frac{R_{\mathrm{n}}}{10^{13} \mathrm{~cm}}\right)\left(\frac{v_{\mathrm{n}}}{10^{9} \mathrm{~cm} \mathrm{~s}^{-1}}\right)^{-1} \mathrm{~s}
$$

debe ser menor que el tiempo de vida de la nube dentro del jet. Para estimar esta escala temporal debemos conocer la velocidad del choque en la nube, $v_{\text {cn }}$, que se obtiene, como vimos en el Capítulo 2, igualando las presiones cinéticas del jet y de la nube: $\left(\Gamma_{\mathrm{j}}-1\right) n_{\mathrm{j}} m_{p} c^{2}=n_{\mathrm{n}} m_{p} v_{\mathrm{cn}}^{2}$, válido mientras $v_{\mathrm{cn}} \ll c$. Así obtenemos

$$
v_{\mathrm{cn}} \sim \frac{c\left(\Gamma_{\mathrm{j}}-1\right)}{\chi_{\mathrm{agn}}^{1 / 2}} \sim 3 \times 10^{8}\left(\frac{n_{\mathrm{n}}}{10^{10} \mathrm{~cm}^{-3}}\right)^{-1 / 2}\left(\frac{z_{\mathrm{j}}}{10^{16} \mathrm{~cm}}\right)^{-1}\left(\frac{L_{\mathrm{j}}}{10^{44} \mathrm{erg} \mathrm{s}^{-1}}\right)^{1 / 2} \frac{\mathrm{cm}}{\mathrm{s}},
$$

donde $\chi_{\text {agn }}=n_{\mathrm{n}} / n_{\mathrm{j}}\left(z_{\mathrm{j}}\right)$. Luego, el tiempo de cruce del choque a través de toda la nube resulta

$$
t_{\mathrm{cn}} \sim \frac{2 R_{\mathrm{n}}}{v_{\mathrm{cn}}} \simeq 7 \times 10^{4}\left(\frac{R_{\mathrm{n}}}{10^{13} \mathrm{~cm}}\right)\left(\frac{n_{\mathrm{n}}}{10^{10} \mathrm{~cm}^{-3}}\right)^{1 / 2}\left(\frac{z_{\mathrm{j}}}{10^{16} \mathrm{~cm}}\right)\left(\frac{L_{\mathrm{j}}}{10^{44} \mathrm{erg} \mathrm{s}^{-1}}\right)^{-1 / 2} \mathrm{~s} .
$$




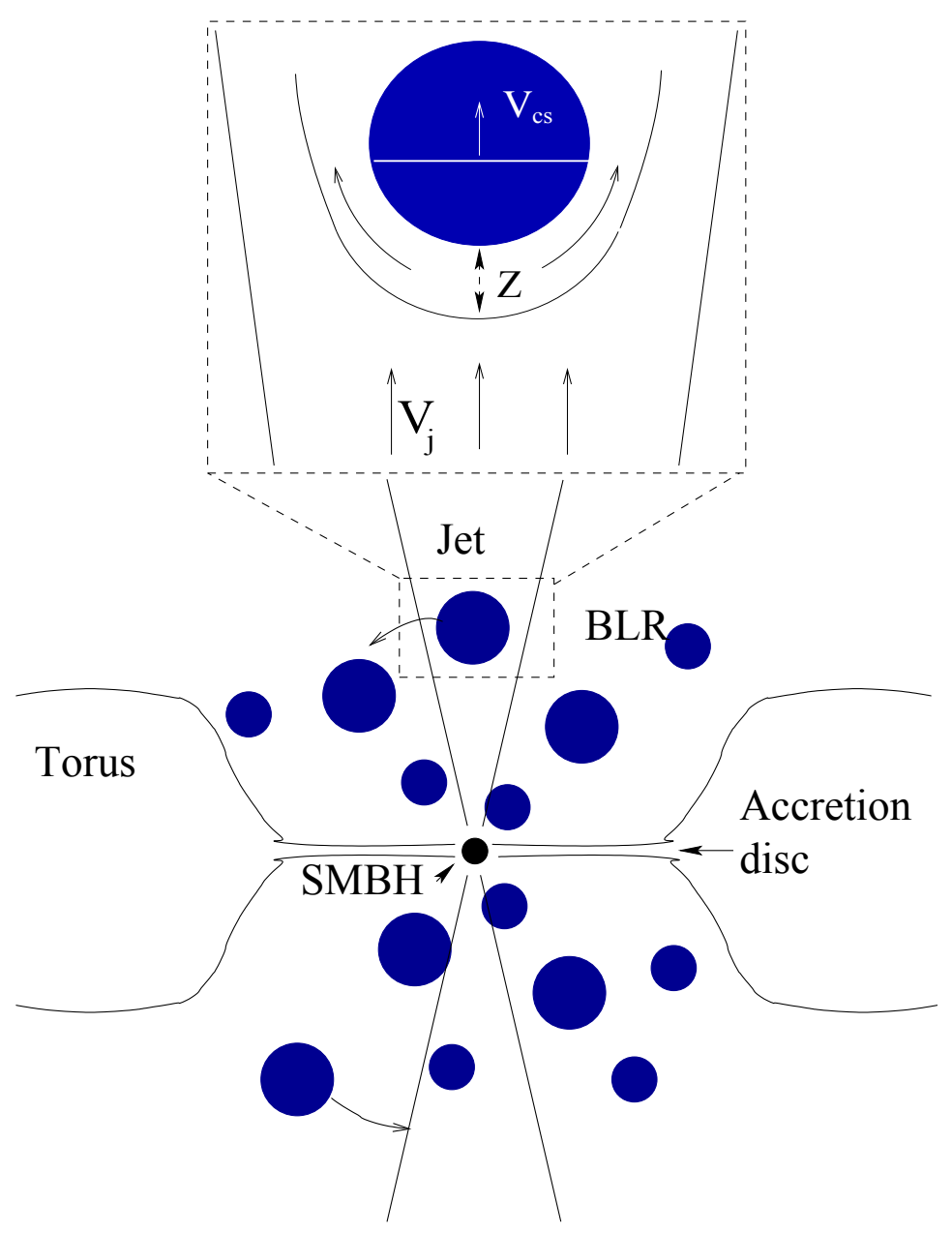

Figura 6.3: Esquema, no a escala, del modelo unificado de los AGN a las escalas espaciales de la BLR. La interacción entre una nube de la BLR y uno de los jets se muestra en la parte superior de la figura. El bow shock en el jet y el choque en la nube también son esquematizados. 
Para un tiempo tan corto como $\sim t_{\mathrm{cn}}$, la nube se comporta como un obstáculo efectivo para el material del jet. Fijando $t_{\mathrm{n}} \sim t_{\mathrm{cn}}$ nos permite obtener valores mínimos para $\chi_{\text {agn }} \mathrm{y}$ $z_{\mathrm{j}}$, por debajo de los cuales la nube no llega a penetrar efectivamente en el jet.

Debido a la interacción con el material del jet, inestabilidades hidrodinámicas afectan a la nube. El jet ejerce una fuerza en la nube a través de la superficie de discontinuidad. La aceleración aplicada a la nube puede estimarse a través de la ecuación (2.49) y en el caso que estamos estudiando resulta

$$
g=\frac{3}{2} \frac{v_{\mathrm{cn}}}{t_{\mathrm{cn}}}=\frac{3}{4} \frac{v_{\mathrm{cn}}^{2}}{R_{\mathrm{n}}} .
$$

Dada la aceleración $g$, las inestabilidades de RT se desarrollarán en la nube con una escala de tiempo

$$
t_{\mathrm{RT}} \sim \sqrt{\frac{l}{g}}=\sqrt{\frac{4 \chi_{\mathrm{agn}} l R_{\mathrm{n}}}{3 c^{2}}} .
$$

Para perturbaciones con longitudes de escala $l \sim R_{\mathrm{n}}$, que son aquellas asociadas a la fragmentación significativa de la nube, el tiempo de crecimiento de la inestabilidad resulta $t_{\mathrm{RT}} \sim t_{\mathrm{cn}}$. Por otro lado, las inestabilidades de $\mathrm{KH}$ también pueden crecer suficientemente como para destruir la nube. Dada la alta velocidad relativa entre el jet chocado y el material de la nube, $v_{\text {rel }} \sim v_{\mathrm{j}}$, se obtiene

$$
t_{\mathrm{KH}} \sim=\frac{\chi_{\mathrm{agn}} l}{c} .
$$

Para $l \sim R_{\mathrm{n}}$, obtenemos nuevamente que $t_{\mathrm{KH}} \sim t_{\mathrm{cn}}$. Notamos que, dada la ecuación (6.5), el tiempo necesario para acelerar la nube hasta la velocidad del choque $v_{\mathrm{cn}}$ es $\sim t_{\mathrm{cn}}$, mientras que para acelerar la nube hasta $v_{\mathrm{j}}$ el tiempo requerido es $\gg t_{\mathrm{cn}}$. Por esto, antes de que la nube comience a moverse conjuntamente con el jet, probablemente será fragmentada.

Finalmente, hay dos escalas de tiempo adicionales que también son relevantes en nuestro estudio: el tiempo de formación del bow shock, $t_{\mathrm{bs}}$, y el tiempo requerido para que la nube cruce el jet, $t_{\mathrm{j}}$. Considerando las ecuaciones (2.45) y (2.46), y que en el caso de un bow shock relativista éste se separa del obstáculo una distancia $Z \sim 0.3 R_{\mathrm{n}}$ (obtenido considerando que en un plasma relativista las partículas se escapan de la región chocada del jet a la velocidad del sonido $c / \sqrt{3}$ ), estas escalas de tiempo resultan

$$
t_{\mathrm{j}} \sim \frac{2 R_{\mathrm{j}}}{v_{\mathrm{n}}}=2 \times 10^{6}\left(\frac{z_{\mathrm{j}}}{10^{16} \mathrm{~cm}}\right)\left(\frac{v_{\mathrm{n}}}{10^{9} \mathrm{~cm} \mathrm{~s}^{-1}}\right)^{-1} \mathrm{~s},
$$

y

$$
t_{\mathrm{bs}} \sim \frac{Z}{c}=10^{2}\left(\frac{R_{\mathrm{n}}}{10^{13} \mathrm{~cm}}\right) \mathrm{s} .
$$

A modo de resumen del estudio anterior sobre la dinámica de la interacción jet-nube, graficamos en la Figura 6.4 $t_{\mathrm{cn}}$ (para diferentes valores de $L_{\mathrm{j}}$ ), $t_{\mathrm{j}}, t_{\mathrm{n}}$ y $t_{\mathrm{bs}}$ en función de $z_{\mathrm{j}}$. Como se muestra en la figura, para algunos valores de $z_{\mathrm{j}}$ y $L_{\mathrm{j}}$ la nube podría ser destruida por el jet antes de entrar completamente, es decir, $t_{\mathrm{cn}}<t_{\mathrm{n}}$. Esto nos provee de una 


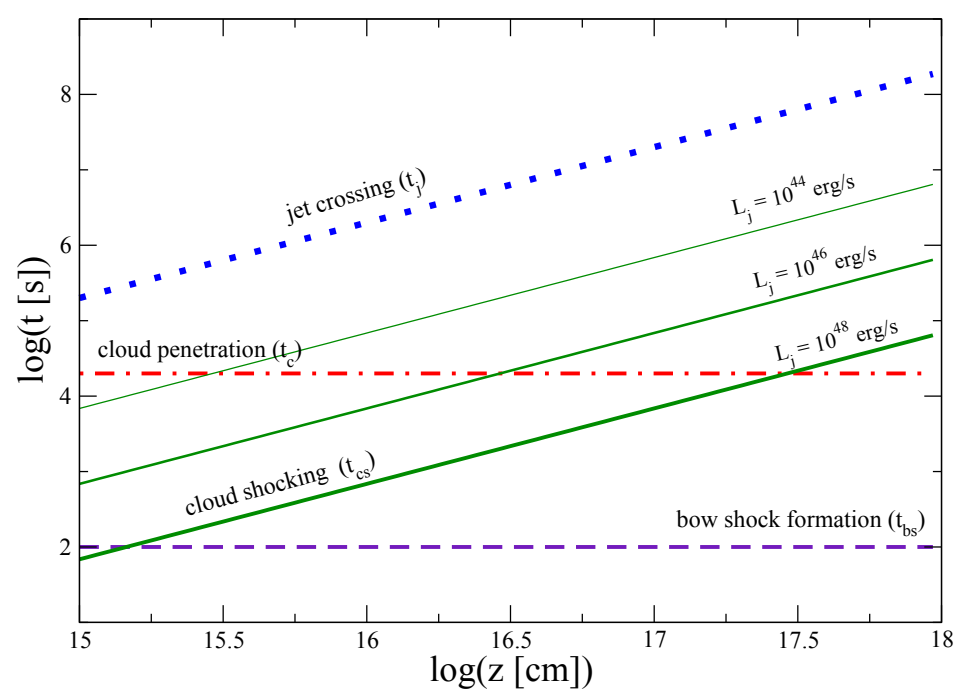

Figura 6.4: Los tiempos de cruce de la nube a través del jet (línea punteada azul), de penetración de la nube (línea punteada-rayada roja), de formación de bow-shock (línea rayada violeta) y el tiempo en el cual el choque en la nube la recorre entera (líneas verdes) son graficados. Todos ellos han sido calculados usando los valores dados en la Tabla 6.1.

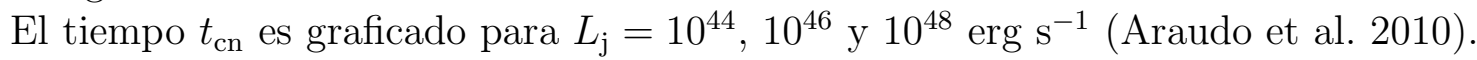

condición para determinar la altura del jet a la cual la nube puede penetrar entera dentro de él. Notamos también que, en general, $t_{\mathrm{bs}}$ es mucho más corto que las demás escalas de tiempo.

\section{Altura de la interacción}

La nube puede penetrar completamente en el jet si el tiempo de vida de la nube después del impacto con el jet es más largo que el tiempo de penetración. Además, la condición de que la presión lateral del jet sea $<n_{\mathrm{n}} m_{p} v_{\mathrm{n}}^{2}$ se satisface automáticamente. Esto determina la altura de interacción mínima, $z_{\text {int }}^{\min }$, que no permite la destrucción de la nube antes de la penetración completa. Por otro lado, la interacción no puede ocurrir más abajo que la región de formación del jet, que se da en $z_{0} \sim 100 R_{\mathrm{g}} \approx 1.5 \times 10^{15}\left(M_{\mathrm{smbh}} / 10^{8} M_{\odot}\right) \mathrm{cm}$ (Junor et al. 1999), donde $R_{\mathrm{g}}$ es el radio gravitacional del agujero negro. Finalmente, para que la interacción entre nubes de la BLR y los jets pueda occurrir, a una altura que llamaremos $z_{\text {int }}$, el tamaño de la BLR debe ser $R_{\text {blr }}>z_{\text {int }}>z_{0}$.

El tiempo de vida de la nube depende del tiempo de fragmentación (dado por el crecimiento de las inestabilidades), que está fuertemente relacionado con $t_{\mathrm{cn}}$. El valor de $z_{\text {int }}$ puede luego ser estimado fijando $t_{\mathrm{n}}<t_{\mathrm{cn}}$, ya que queremos que la nube entre completa- 
mente en el jet antes de ser sustancialmente distorcionada por el impacto con éste. Una vez chocada, la nube puede sufrir expansión lateral y calentamiento por conducción lo cual haría que las inestabilidades crezcan más rápidamente. Sin embargo no hemos tenido en cuenta estos dos últimos efectos en nuestro estudio y determinamos $z_{\text {int }}$ imponiendo que $t_{\mathrm{cn}}=2 t_{\mathrm{n}}$, con lo cual

$$
z_{\mathrm{int}} \approx 5 \times 10^{15}\left(\frac{v_{\mathrm{n}}}{10^{9} \mathrm{~cm} \mathrm{~s}^{-1}}\right)^{-1}\left(\frac{n_{\mathrm{n}}}{10^{10} \mathrm{~cm}^{-3}}\right)^{-1 / 2}\left(\frac{L_{\mathrm{j}}}{10^{44} \mathrm{erg} \mathrm{s}^{-1}}\right)^{1 / 2} \mathrm{~cm} .
$$

Notamos que la potencia disponible en el bow shock es $L_{\mathrm{bs}} \sim\left(\sigma_{\mathrm{n}} / \sigma_{\mathrm{j}}\right) L_{\mathrm{j}} \propto z_{\mathrm{j}}^{-2}$, donde $\sigma_{\mathrm{n}}=\pi R_{\mathrm{n}}^{2}$, por lo cual la interacción más luminosa tendrá lugar en $z_{\mathrm{j}} \sim z_{\text {int }}$. Ahora debemos verificar si la elección de $z_{\text {int }}$ que hemos hecho cumple con los requisitos geométricos $z_{0}<$ $z_{\text {int }}<R_{\text {blr }}$.

El tamaño de la BLR puede estimarse a través de las relaciones empíricas obtenidas para galaxias FR II, las cuales poseen una BLR bien determinada por las observaciones. Los ajustes que se obtienen a partir de las observaciones de numerosas galaxias son en general del tipo $R_{\mathrm{blr}} \propto L_{\mathrm{blr}}^{\alpha_{\mathrm{blr}}}$, donde $L_{\mathrm{blr}}$ es la luminosidad de la BLR y $\alpha_{\mathrm{blr}} \sim 0.5-0.7$ (Peterson et al. 2005; Bentz et al. 2006). En este capítulo usamos las siguientes relaciones:

$$
R_{\mathrm{blr}} \sim 6 \times 10^{16}\left(\frac{L_{\mathrm{blr}}}{10^{44} \mathrm{erg} \mathrm{s}^{-1}}\right)^{0.7} \mathrm{~cm},
$$

y

$$
R_{\mathrm{blr}} \sim 2.5 \times 10^{16}\left(\frac{L_{\mathrm{blr}}}{10^{44} \mathrm{erg} \mathrm{s}^{-1}}\right)^{0.55} \mathrm{~cm},
$$

obtenidas por Kaspi y colaboradores (2005, 2007). En galaxias FR I, en las cuales la detección de la BLR es muy imprecisa o ni siquiera se llega a detectar, las relaciones (6.11) y (6.12) deben tomarse con cautela.

En la Figura 6.5 mostramos como varían $z_{\text {int }}$ y $R_{\text {blr }}$ con $L_{\mathrm{j}}$, asumiendo que $L_{\mathrm{blr}}$ es un $10 \%$ de la luminosidad del disco, $L_{\mathrm{d}}$, y esta última es tomada igual a $L_{\mathrm{j}}$. Como puede verse en la figura, para valores razonables de los parámetros, la condición $z_{\text {int }}<R_{\text {blr }}$ se satisface en un amplio rango de valores de $L_{\mathrm{j}}$. En la misma figura también mostramos la relación entre $z_{0}$ y $M_{\mathrm{smbh}}$, de la cual se deduce que para $M_{\mathrm{smbh}}>10^{9} M_{\odot}$ el jet podría no estar (completamente) formado a las escalas de la BLR para valores bajos de $L_{\mathrm{j}}$.

\subsection{Población de partículas relativistas}

En el bow shock y en el choque en la nube se pueden acelerar partículas a través de un mecanismo de aceleración difusiva como el descripto en el Capítulo 2 (Sección 2.6). Sin embargo, debido a que el bow shock es más fuerte que el choque en la nube $\left(v_{\mathrm{bs}} \gg v_{\mathrm{cn}}\right)$, la aceleración de partículas será más eficiente en el primero. Además, la luminosidad del choque en la nube es menor que la luminosidad del bow shock por un factor $\sim 1 /\left(2 \chi_{\text {agn }}^{1 / 2}\right)$, con lo cual la energía disponible para entregar a las partículas aceleradas es menor también. Por 


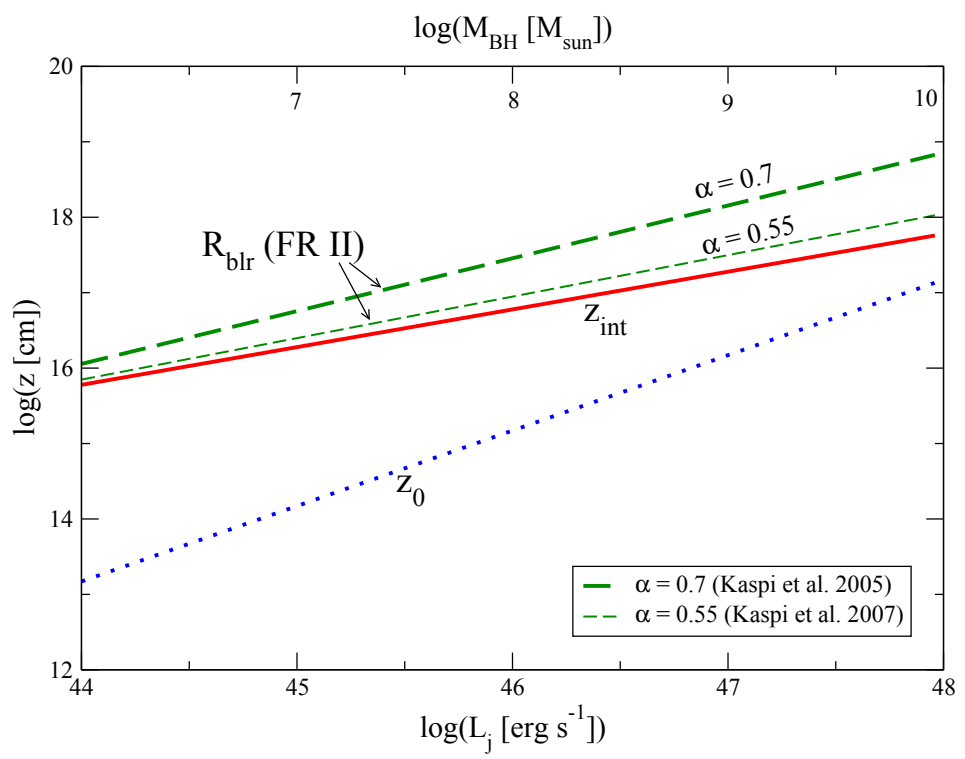

Figura 6.5: La altura de interacción, $z_{\text {int }}$ (línea sólida roja), y el tamaño de la BLR, $R_{\text {blr }}$ (línea rayada verde), se grafican para diferentes valores de $L_{\mathrm{j}}$ (eje horizontal inferior). Derivamos $R_{\mathrm{blr}}\left(L_{\mathrm{j}}\right)$ fijando que $L_{\mathrm{blr}}=0.1 L_{\mathrm{j}}$ y graficamos $R_{\mathrm{blr}}$ usando las ecuaciones (6.11) y (6.12). En la misma figura, la altura de la base del jet, $z_{0}$ (línea punteada azul), es graficada en función de $M_{\text {smbh }}$ (eje horizontal superior). 
estas razones, sólo consideraremos la aceleración de electrones y protones en el bow shock y en esta sección describiremos someramente la inyección y evolución de estas partículas no térmicas.

La luminosidad inyectada por el jet en el bow shock localizado en $z_{\mathrm{int}}$ es $L_{\mathrm{bs}}=\left(\sigma_{\mathrm{n}} / \sigma_{\mathrm{j}}\right) L_{\mathrm{j}}$. Una fracción $\eta_{\text {nt }}$ de esta cantidad será transferida a las partículas que se aceleran en este choque, con lo cual la luminosidad de éstas es

$$
L_{e, p}=\eta_{\mathrm{nt}} L_{\mathrm{bs}} \approx 4 \times 10^{39}\left(\frac{\eta_{\mathrm{nt}}}{0.1}\right)\left(\frac{R_{\mathrm{n}}}{10^{13} \mathrm{~cm}}\right)^{2}\left(\frac{L_{\mathrm{j}}}{10^{44} \mathrm{erg} \mathrm{s}^{-1}}\right) \frac{\mathrm{erg}}{\mathrm{s}} .
$$

La distribución de energía de la población de partículas relativistas inyectadas en la región del bow shock es una ley de potencias con un cut-off exponencial:

$$
Q_{e, p}=K_{e, p} E_{e, p}^{-2.2} \exp ^{-E_{e, p} / E_{e, p}^{\max }} .
$$

A la constante $K_{e, p}$ la determinamos a través de la ecuación (6.13) y considerando que

$$
L_{e, p}=\int_{E_{e, p}^{\min }}^{E_{e, p}^{\max }} Q_{e, p} E_{e, p} \mathrm{~d} E_{e, p},
$$

donde fijamos $E_{e, p}^{\min }=2 m_{e, p} c^{2}$ pero $E_{e, p}^{\max }$ es desconocida aún. Sin embargo, igualando las ecuaciones (6.14) y (6.15) obtenemos

$$
K_{e, p}=\eta_{\mathrm{nt}} L_{\mathrm{bs}}\left(\frac{0.2}{E_{e, p}^{\min -0.2}-E_{e, p}^{\max }-0.2}\right) \sim \eta_{\mathrm{nt}} L_{\mathrm{bs}} \frac{0.2}{E_{e, p}^{\min -0.2}},
$$

si $E_{e, p}^{\max } \gg E_{e, p}^{\min }$.

\subsubsection{Aceleración de partículas y pérdidas radiativas}

El campo magnético $B_{\text {bs }}$ en el acelerador/emisor puede determinarse relacionando las densidades de energía magnética y no térmica de la siguiente manera: $u_{\mathrm{B}}=\eta_{\mathrm{B}} u_{\mathrm{e}, \mathrm{p}}$, donde la densidad de energía de las partículas relativistas es $u_{e, p}=L_{e, p} /\left(\sigma_{\mathrm{n}} c\right)$. De manera tal que la radiación IC sea importante en rayos gamma, $\eta_{\mathrm{B}} \ll 1$ es requerido. En este contexto, si $\eta_{\mathrm{B}}=0.01, B_{\mathrm{bs}}$ puede se parametrizado de la siquiente manera:

$$
B_{\mathrm{bs}} \approx 10\left(\frac{\eta_{\mathrm{B}}}{0.01}\right)\left(\frac{v_{\mathrm{n}}}{10^{9} \mathrm{~cm} \mathrm{~s}^{-1}}\right)^{2}\left(\frac{n_{\mathrm{n}}}{10^{10} \mathrm{~cm}^{-3}}\right) \mathrm{G} .
$$

Debido a que el bow shock es relativista y el tratamiento de estos choques es complejo, adoptamos la prescripción $\dot{E}_{e, p}^{\mathrm{ac}} \sim 0.1 e B_{\mathrm{bs}} c \operatorname{erg~s}^{-1}$ (de Jager et al. 1996) para la tasa de aceleración, con la cual el tiempo de aceleración está dado por la relación

$$
t_{\mathrm{ac}} \sim 0.7 E_{e, p}\left(\frac{B_{\mathrm{bs}}}{10 \mathrm{G}}\right)^{-1} \mathrm{~s} .
$$


Las partículas están afectadas por diferentes tipos de pérdidas que compensan las ganancias por aceleración. Las pérdidas por escape de las partículas contemplan la convección del material chocado,

$$
t_{\mathrm{conv}} \sim \frac{3 R_{\mathrm{n}}}{c}=10^{3}\left(\frac{R_{\mathrm{n}}}{10^{13} \mathrm{~cm}}\right) \mathrm{s},
$$

y la difusión, la cual para que las partículas puedan difundir una distancia $Z=0.3 R_{\mathrm{n}}$ el tiempo requerido es

$$
t_{\mathrm{dif}} \sim 5 \times 10^{6}\left(\frac{R_{\mathrm{n}}}{10^{13} \mathrm{~cm}}\right)^{2}\left(\frac{B_{\mathrm{bs}}}{10 \mathrm{G}}\right) \frac{1}{E_{e, p}} \mathrm{~s} .
$$

\section{Pérdidas leptónicas}

Los electrones en la región chocada del jet radían principalmente por los procesos Bremsstrahlung relativista, radiación sincrotrón y dispersiones IC. En el último caso los fotones semilla pueden ser externos (EC), producidos en el disco o en la BLR, o generados en la misma fuente por radiación sincrotrón (SSC). Dada la baja densidad del jet en $z_{\text {int }}$ $\left(n_{\mathrm{j}} \sim 3.2 \times 10^{5} \mathrm{~cm}^{-3}\right)$ las pérdidas por Bremsstrahlung relativista serán despreciables, con una escala de tiempo $t_{\mathrm{Brem}} \sim 10^{10} \mathrm{~s}$. Considerando el valor de $B_{\mathrm{bs}}$ dado en la ecuación (6.17), las pérdidas por radiación sincrotrón resultan:

$$
t_{\mathrm{sin}} \sim 4.1\left(\frac{\eta_{\mathrm{B}}}{0.01}\right)^{-2} \frac{1}{E_{e}} \mathrm{~s} .
$$

Para estimar la relevancia de las interacciones IC (EC o SSC), necesitamos conocer la densidad $n_{\mathrm{ph}}$ de fotones semilla para así luego calcular $t_{\mathrm{EC}} \mathrm{y} t_{\mathrm{SSC}}$ a través de las fórmulas dadas en el Capítulo 3. A continuación describimos como calculamos $n_{\mathrm{ph}}$ de cada campo de fotones semilla considerado en este capítulo.

- SSC: Estimando la luminosidad de la radiación sincrotrón, $L_{\mathrm{sin}} \sim \int N_{e}\left(E_{e}\right) P_{\sin }\left(E_{e}, E_{\mathrm{ph}}\right) \mathrm{d} E_{e}$, donde $\mathcal{P}\left(E_{e}, E_{\mathrm{ph}}\right)$ está dada por la fórmula (3.12) y considerando que la distribución $N_{e}$ se encuentra en el estado estacionario, podemos hallar la densidad de energía de los fotones sincrotrón: $u_{\sin } \sim L_{\sin } /\left(\sigma_{\mathrm{n}} c\right)$. Como la distribución de estos fotones es una ley de potencia, la densidad de fotones es $n_{\mathrm{ph}}^{\mathrm{sin}}=\mathrm{d} U_{\mathrm{sin}} / \mathrm{d} E_{0}^{\mathrm{sin}}$. Para calcular $t_{\mathrm{SSC}}$ desarrollamos un código numérico que describiremos someramente más abajo.

- EC (disco): La densidad de energía $u_{\mathrm{d}}$ de los fotones emitidos por el disco puede estimarse de la siguiente manera: $u_{\mathrm{d}} \sim L_{\mathrm{d}} /\left(4 \pi z_{\text {int }}^{2} c\right)$. De acuerdo al modelo de disco delgado de Shakura \& Sunyaev (1973), la temperatura $T_{\mathrm{d}}$ del material acretado decrece con la distancia $r_{\mathrm{d}}$ al SMBH de acuerdo a $T_{\mathrm{d}}\left(r_{\mathrm{d}}\right)=T_{\text {in }}\left(r_{\mathrm{d}} / r_{\text {in }}\right)^{-3 / 4}$, donde $r_{\text {in }}=3 r_{\mathrm{g}}=4.5 \times 10^{5}\left(M_{\mathrm{smbh}} / M_{\odot}\right) \mathrm{cm} \mathrm{y} T_{\text {in }}=T_{\mathrm{d}}\left(r_{\text {in }}\right)$. Debido a que la mayor intensidad de radiación se produce en las partes más internas del disco, esto es, en $r_{\mathrm{d}} \sim r_{\text {in }}$, podemos considerar que $T_{\mathrm{d}} \sim T_{\text {in }}$ y luego $L_{\mathrm{d}} \sim 4 \pi \sigma_{S B} T_{\mathrm{in}}^{4} r_{\text {in }}^{2}$, donde $\sigma_{\mathrm{SB}}=5.67 \times 10^{-5} \mathrm{gr} \mathrm{K}{ }^{-4} \mathrm{~s}^{-2}$. Despejando de aquí la temperatura, la energía de los fotones emitidos por el disco resulta $E_{0}^{\mathrm{d}} \sim 2.4 \times 10^{2}\left(L_{\mathrm{d}} / 10^{44}\right)^{1 / 4}\left(M_{\mathrm{smbh}} / 10^{6} M_{\odot}\right)^{-1 / 2} \mathrm{eV}$ 
y los electrones con $E_{e}>\left(L_{\mathrm{d}} / 10^{44}\right)^{-1 / 4}\left(M_{\mathrm{smbh}} / 10^{6} M_{\odot}\right)^{1 / 2} \mathrm{GeV}$ interactúan con los fotones del disco en el régimen de $\mathrm{KN}$.

- EC (BLR): La densidad de energía de los fotones emitidos por la BLR es calculada mediante $u_{\mathrm{blr}} \sim L_{\mathrm{blr}} /\left(\pi R_{\mathrm{blr}}^{2} c\right) \sim 0.08\left(L_{\mathrm{blr}} / 10^{44}\right)^{-0.4}$, donde hemos considerado la relación (6.11). Para nubes con temperaturas $T_{\mathrm{n}} \sim 2 \times 10^{4} \mathrm{~K}$, la energía de los fotones resulta $E_{0}^{\mathrm{blr}} \sim 5.3 \mathrm{eV}$ y la interacción en el régimen de $\mathrm{KN}$ se dará para dispersiones con electrones cuya energía sea $E_{e}>48 \mathrm{GeV}$.

En la Figura 6.6 (izquierda) se muestran las escalas de tiempo de las pérdidas radiativas, junto con el tiempo de aceleración y de convección para un bow shock ubicado en $z_{\text {int }}$. Para $\eta_{\mathrm{B}}$ adoptamos un valor igual a 0.01 y las pérdidas por SSC son graficadas para el estado estacionario de $N_{E_{e}}\left(E_{e}\right)$. Notamos que tanto $B_{\mathrm{bs}}$ como $L_{e, p}$ y $z_{\text {int }}$ son constantes para diferentes valores de $L_{\mathrm{j}}$ y fijadas las fracciones $\eta_{\mathrm{B}}$ y $\eta_{\mathrm{nt}}$. Sólo $L_{\mathrm{blr}}$ y $L_{\mathrm{d}}$ se espera que varien con $L_{\mathrm{j}}$. Así, mientras los campos de fotones externos no sean relevantes, la energía máxima que pueden alcanzar los electrones en $z_{\text {int }}$ no cambia para diferentes potencias del jet.

Los procesos radiativos dominantes a las energías más altas de la población de electrones son el sincrotrón, el EC y el SSC. El Bremsstrahlung relativista es despreciable para todas las energías y el escape por convección es relevante en la parte de más bajas energías. Esto produce un quiebre en el espectro de los electrones, cuando las pérdidas por sincrotrón, EC o SSC se igualan a las de convección. La transición del régimen de Th al de KN se ve claramente en las curvas de enfriamiento por EC, pero es menos notorio en el caso del SSC debido a la naturaleza no térmica de los fotones semilla (sincrotrón). La energía máxima de los electrones es de $\sim 40 \mathrm{TeV}$ (para $\eta_{\mathrm{B}}=0.01$ ) y está determinada por la igualdad $t_{\mathrm{ac}}=t_{\mathrm{sin}}$.

A primer orden, la evolución de la distribución de los electrones relativistas en la región del bow shock puede ser calculada asumiendo condiciones homogéneas en el acelerador/emisor y los mecanismos de aceleración y escape mencionados anteriormente. Debido a que el SSC es un canal de enfriamiento importante, el cálculo de $N_{e}\left(E_{e}, t\right)$ debe realizarse numéricamente. Para esto desarrollamos un código en el cual para un tiempo de observación $t$, dividimos el período $[0-t]$ en intervalos $\Delta t$. En cada intervalo, $N_{e}$ se calcula teniendo en cuenta las pérdidas por SSC con los fotones sincrotrón radiados en el intervalo anterior. Así se continúa hasta que se alcanza el estado estacionario. La duración de cada intervalo debe ser menor que la duración de los intervalos previos de modo de calcular apropiadamente el crecimiento de $u_{\text {sin }}$ en el emisor. En la Figura 6.6 (derecha) se muestra el resultado obtenido considerando $\tau_{\text {vida }}>t_{\mathrm{cn}}$. El estado estacionario se alcanza cuando $t=t_{\mathrm{conv}}$.

\section{Pérdidas hadrónicas}

Debido a la baja densidad del jet en $z_{\text {int }}$, los protones no se enfrían eficientemente en la región del bow shock por interacciones $p p$. Considerando que $n_{\mathrm{j}}\left(z_{\text {int }}\right) \sim 3.2 \times 10^{5} \mathrm{~cm}^{-3}$, el tiempo de enfriamiento por $p p$ resulta muy largo, $t_{p p}^{\mathrm{bs}} \sim 2 \times 10^{9} \mathrm{~s}$. La energía máxima de 

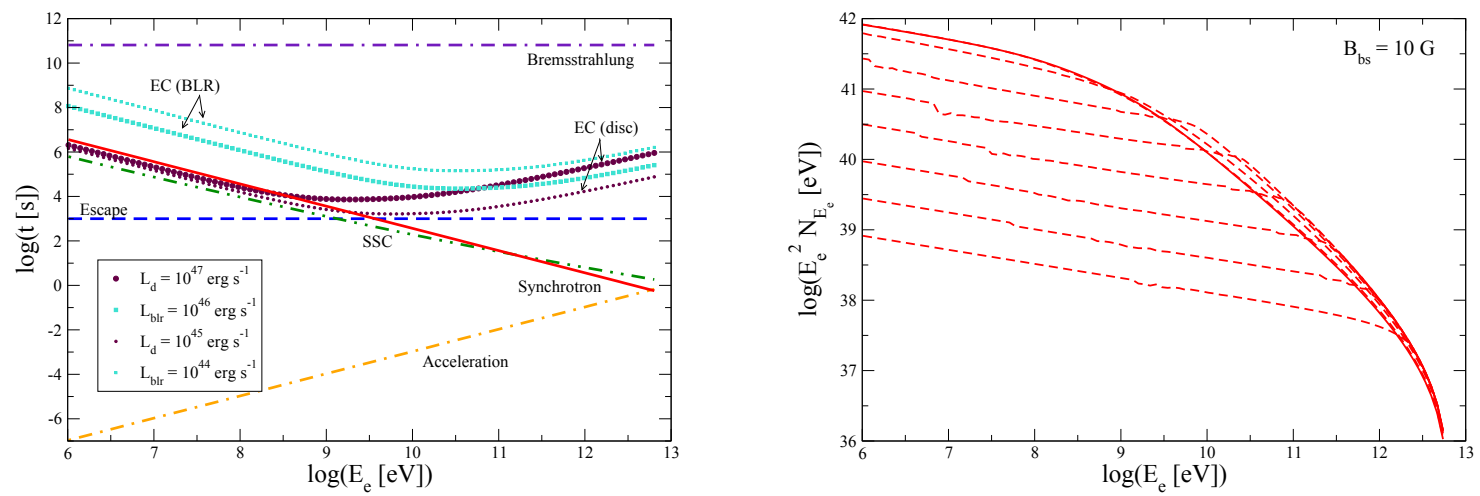

Figura 6.6: Izquierda: Las ganancias por aceleración (línea punteada-rayada anaranjada), convección (línea rayada azul) y los tiempos de enfriamiento radiativos son graficados. El tiempo SSC (línea verde de rayas y puntos) graficado corresponde al calculado con la distribución de energía estacionaria de los electrones. El tiempo de enfriamiento por EC con los fotones de la BLR (cuadrados turquesa) y del disco (línea punteada marrón) son graficados para fuentes débiles (BLR: $10^{44} \mathrm{erg} \mathrm{s}^{-1}$; disco: $10^{45} \mathrm{erg} \mathrm{s}^{-1}$ ) y brillantes (BLR: $10^{46} \mathrm{erg} \mathrm{s}^{-1}$; disco: $10^{47} \mathrm{erg} \mathrm{s}^{-1}$ ). Las pérdidas por radiación sincrotrón (línea roja) y Bremsstrahlung relativista (línea violeta) son también graficadas. Hemos fijado $\eta_{\mathrm{B}}=0.01$. Derecha: Evolución temporal de la distribución de los electrones relativistas en la región del bow shock, $N_{e}$. Las líneas de rayas indican el espectro a diferentes tiempos $t_{i}$, mientras que la línea llena indica el espectro una vez que el estado estacionario ha sido alcanzado. Como puede observarse, el quiebre en el espectro estacionario se produce en $E_{e} \sim 2 \times 10^{9} \mathrm{eV}$, como se deduce de la figura de la izquierda. 
los protones queda entonces determinada por el tiempo que tardan éstos en difundir una distancia $Z=0.3 R_{\mathrm{n}}$ desde el bow shock hasta la nube, lo cual nos da una energía máxima

$$
E_{p}^{\max } \sim 0.1 e B_{\mathrm{bs}} R_{\mathrm{n}}=5 \times 10^{3}\left(\frac{B_{\mathrm{bs}}}{10 \mathrm{G}}\right)\left(\frac{R_{\mathrm{n}}}{10^{13} \mathrm{~cm}}\right) \mathrm{TeV}
$$

Los protones con energías $E_{p}>0.4 E_{p}^{\max }$, es decir, $t_{\text {conv }}>t_{\text {dif }}$, podrán difundir hasta la nube antes de ser convectados por el material chocado del jet.

En la nube, el campo magnético necesario para confinar a estos protones tan energéticos es $B_{\mathrm{n}}^{\text {conf }} \geq 0.7\left(B_{\mathrm{bs}} / 10 \mathrm{G}\right)$. Si los protones estuviesen confinados en la nube, entonces radiarían una fracción significativa de su energía por interacciones $p p$ con el material chocado de la nube en un tiempo $t_{p p} \sim 2 \times 10^{15} /\left(4 n_{\mathrm{n}}\right) \sim 5 \times 10^{4} \mathrm{~s}$. Sin embargo, como no conocemos el valor del campo magnético de las nubes de la BLR, adoptamos que las partículas solo permanecen en la nube el tiempo que tardan en cruzarla a una velocidad $\sim c$, con lo cual el tiempo de cruce es $t_{\text {cruce }} \sim R_{\mathrm{n}} / c \sim 700 \mathrm{~s}<t_{p p}$. Luego, la distribución energética de los protones relativistas en la nube será

$$
N_{p}\left(E_{p}\right) \sim \frac{R_{\mathrm{n}}}{c} Q_{p}\left(E_{p}\right)
$$

donde $Q_{p}=K_{p} E_{p}^{-2.2} \exp \left(-E_{p} / E_{p}^{\max }\right)$ y $K_{p}$ está dada por la ecuación (6.16).

\subsection{Distribuciones espectrales de energía}

Una vez que las distribuciones de energía de las partículas relativistas $\left(N_{e}\left(E_{e}\right)\right.$ y $\left.N_{p}\left(E_{p}\right)\right)$ han sido calculadas (en el estado estacionario), estamos en condiciones de calcular las SEDs de la radiación no térmica.

\subsubsection{Emisión asociada al bow shock}

En el bow shock los electrones pierden energía basicamente por radiación sincrotrón, SSC y EC (con los fotones del disco y de la BLR), aunque este último no es el proceso dominante si $B_{\mathrm{bs}} \sim 10 \mathrm{G}$. La autoabsorción sincrotrón es tenida en cuenta, pero solo afecta a la parte menos energética de los fotones sincrotrón. En rayos gamma, la absorción (gamma-gamma) por creación de pares $e^{ \pm}$producida por la emisión del disco de acreción y de la BLR también debe ser considerada, pero la absorción interna debida a la radiación sincrotrón es despreciable y no la tenemos en cuenta. Dadas las energías típicas de los fotones emitidos por el disco y la BLR, $\sim 1 \mathrm{keV}$ y $\sim 10 \mathrm{eV}$, respectivamente, los rayos gamma con energías por encima de 1 y $100 \mathrm{GeV}$ pueden ser fuertemente afectados por la absorción gamma-gamma. Por otro lado, en la mayoría de los casos los fotones con energías $<1 \mathrm{GeV}$ van a escapar del denso campo de fotones emitidos por el disco.

En la Figura 6.7 se muestra la SED de los procesos sinctrotrón y SSC en la región del bow shock. Debido a que este cálculo fue hecho para un caso general, sin especificar las luminosidades ni del jet, ni de la BLR, ni del disco, la absorción gamma-gamma no 


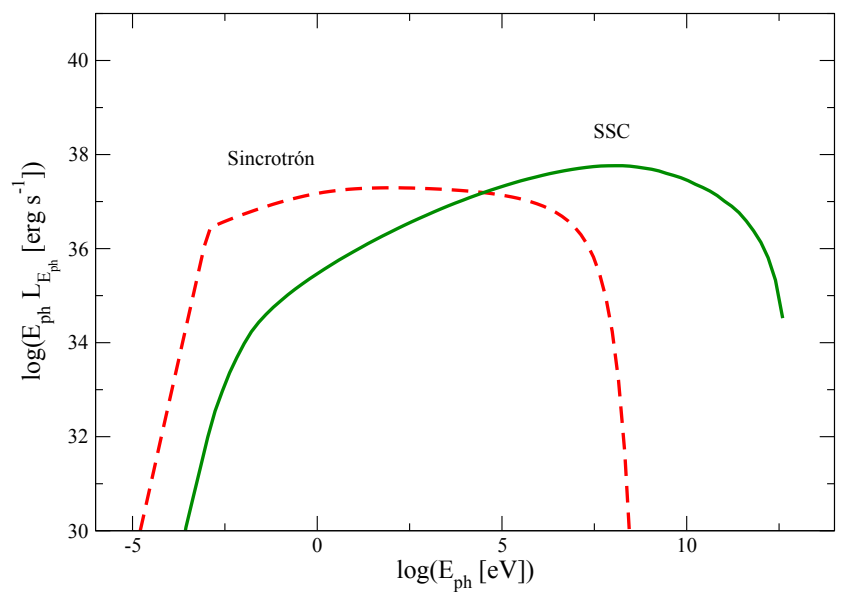

Figura 6.7: Distribución espectral de energía calculada en el caso general considerando los procesos sincrotrón y SSC. Puede observarse el efecto de la autoabsorción sincrotrón, a $E_{\mathrm{ph}}<10^{-3} \mathrm{eV}$.

fue tenida en cuenta, pero sí la autoabsorción sinctrotrón. Las luminosidades bolométricas alcanzadas por ambos procesos de emisión son $L_{\sin } \sim 4 \times 10^{38}$ erg s $^{-1}$ y $L_{\mathrm{SSC}} \sim 8 \times$ $10^{38} \mathrm{erg} \mathrm{s}^{-1}$.

\subsubsection{Emisión asociada a la nube}

Aunque los protones no se enfríen eficientemente en la región del bow shock, sí pueden hacerlo en la nube aquellos que con $E_{p}>0.4 E_{p}^{\max }$ llegan hasta allí. Sin embargo, debido a que estos protones son muy energéticos y el campo magnético necesario para confinarlos en la nube es muy alto, estos protones cruzarán la nube a la velocidad de la luz. Con lo cual, solo la pequeña fracción $\sim 0.17 t_{\text {cruce }} / t_{p p} \sim 10^{-3}$ de la energía promedio por protón será radiada por interacciones $p p$ en la nube y la luminosidad bolométrica emitida resulta $L_{p p} \sim 2 \times 10^{36} \mathrm{erg} \mathrm{s}^{-1}$. Debido a que no hemos considerado confinamiento, este valor es un límite inferior para la emisión por $p p$ de la nube.

\subsection{Interacciones múltiples}

El tamaño de la BLR determina la región en la cual pueden ocurrir interacciones jetnube. Debido a que hay muchas nubes en la BLR, es lícito pensar que algunas de ellas pueden interactuar simultáneamente con el jet a diferentes alturas $z_{\text {int }}^{\min }<z_{\mathrm{j}}<R_{\text {blr }}$ y cada una de las cuales produce una cierta cantidad de radiación no térmica. Esta luminosidad total puede ser mucho mayor que la producida por la interacción de una única nube con 


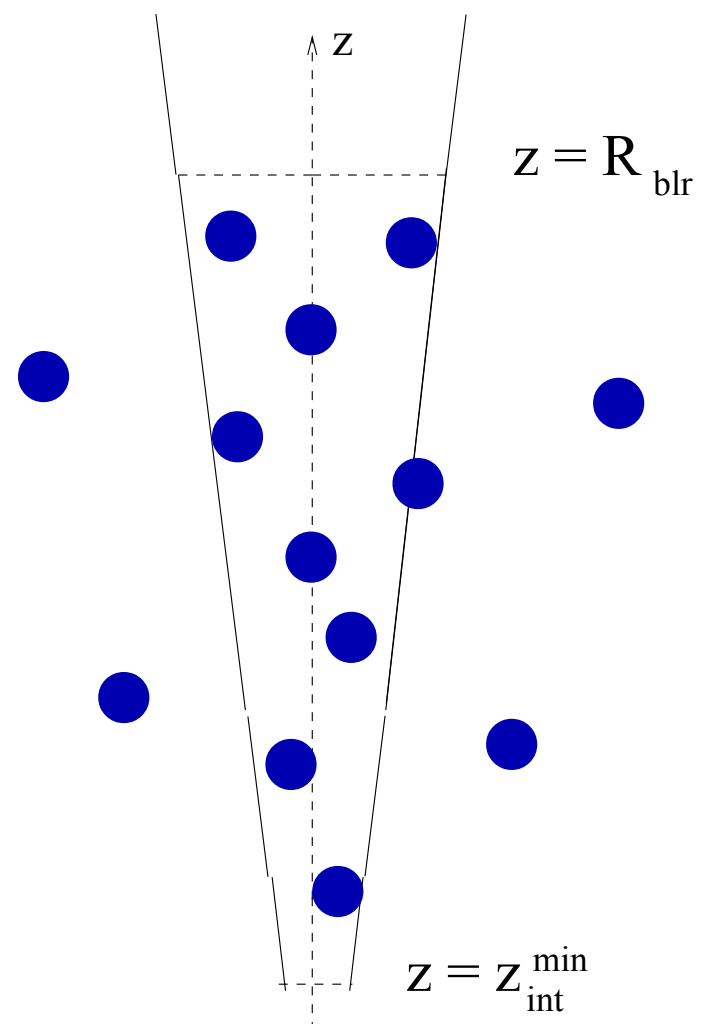

Figura 6.8: Esquema de la interacción simultánea de muchas nubes con el jet. 
el jet, que es $\sim L_{e, p}$. El número de nubes dentro de ambos jets, $N_{\mathrm{n}}^{\mathrm{j}}$, puede ser calculado a través del volumen de cada jet $\left(V_{\mathrm{j}}\right)$ y de cada nube $\left(V_{\mathrm{n}}\right)$, resultando

$$
N_{\mathrm{n}}^{\mathrm{j}}=2 f \frac{V_{\mathrm{j}}}{V_{\mathrm{n}}} \sim 9\left(\frac{L_{\mathrm{j}}}{10^{44} \mathrm{erg} \mathrm{s}^{-1}}\right)^{2}\left(\frac{R_{\mathrm{n}}}{10^{13} \mathrm{~cm}}\right)^{-3},
$$

donde el factor 2 es debido a la presencia de dos jets y $f \sim 10^{-6}$ es el factor de llenado de nubes en la BLR (Dietrich et al. 1999). Este cálculo de $N_{\mathrm{n}}^{\mathrm{j}}$ es correcto si no tenemos en cuenta que las nubes pueden ser destruídas dentro del jet y los fragmentos eventualmente diluídos. Por ejemplo, Klein y colaboradores (1994) estimaron que el tiempo de vida de una nube chocada es varias veces $t_{\mathrm{cn}}$, y Shin y colaboradores (2008) encontraron que aún un campo magnético débil en la nube puede incrementar significativamente el tiempo de vida de éstas. Finalmente, aún en el caso de que las nubes se fragmenten, bow shocks fuertes pueden formarse alrededor de cada fragmento antes de que éstos sean acelerados hasta una velocidad $\sim v_{\mathrm{j}}$. Todas estas consideraciones hacen que el número real de nubes que simultáneamente están interactuando con el jet sea difícil de estimar, pero este número debe estar entre $\left(t_{\mathrm{cn}} / t_{\mathrm{j}}\right) N_{\mathrm{n}}^{\mathrm{j}}$ y $N_{\mathrm{n}}^{\mathrm{j}}$. La presencia de muchas nubes dentro del jet, no solo en $z_{\text {int }}$ sino también a $z_{\mathrm{j}}$ más altos, implica que la luminosidad no térmica total disponible en la intersección entre el jet y la BLR es

$$
L_{e, p}^{\mathrm{tot}} \sim 2 \int^{R_{\mathrm{blr}}} \frac{\mathrm{d} N_{\mathrm{n}}^{\mathrm{j}}}{\mathrm{d} z} L_{\mathrm{nt}}(z) \mathrm{d} z \sim 1.7 \times 10^{40}\left(\frac{\eta_{\mathrm{nt}}}{0.1}\right)\left(\frac{R_{\mathrm{n}}}{10^{13}}\right)^{-1}\left(\frac{L_{\mathrm{j}}}{10^{44} \mathrm{ergs}^{-1}}\right)^{1.7},
$$

donde $\mathrm{d} N_{\mathrm{n}}^{\mathrm{j}}$ es el número de nubes localizadas en un volumen del jet $\mathrm{d} V_{\mathrm{j}}=\pi(0.1 z)^{2} \mathrm{~d} z_{\mathrm{j}}$. En ambas ecuaciones (6.24) y (6.25), $L_{\mathrm{blr}}$ ha sido fijada en $0.1 L_{\mathrm{j}}$, aproximadamente como en las galaxias FR II, y $R_{\mathrm{blr}}$ ha sido derivada usando la ecuación (6.11).

En la Figura 6.9 mostramos estimaciones de la luminosidad en rayos gamma predicha para el caso de la interacción simultánea de muchas nubes con el jet. Para esto hemos seguido un procedimiento muy simple, asumiendo que toda la luminosidad no térmica es radiada en forma de rayos gamma. Este puede ser el caso si los tiempos de escape y de enfriamiento por radiación sincrotrón son más largos que el tiempo de enfriamiento por IC $(\mathrm{EC}+\mathrm{SSC})$ para los electrones más energéticos. Dado que se conoce muy poco de la BLR en las galaxias FR I, no consideramos este tipo de fuentes en la figura.

\subsection{Aplicaciones}

En las próximas dos subsecciones presentamos los resultados obtenidos de aplicar el modelo expuesto en las secciones 6.2 y 6.3 a dos fuentes características: Cen A (FR I, única interacción) y 3C 273 (FR II, múltiples interacciones).

\subsubsection{Galaxias FR I: Cen A}

Cen $\mathrm{A}$ es la galaxia activa más cercana, ubicada a una distacia $d \approx 3.7 \mathrm{Mpc}$ (Israel 1998). Esta fuente ha sido clasificada como una radio-galaxia FR I y como un objeto 


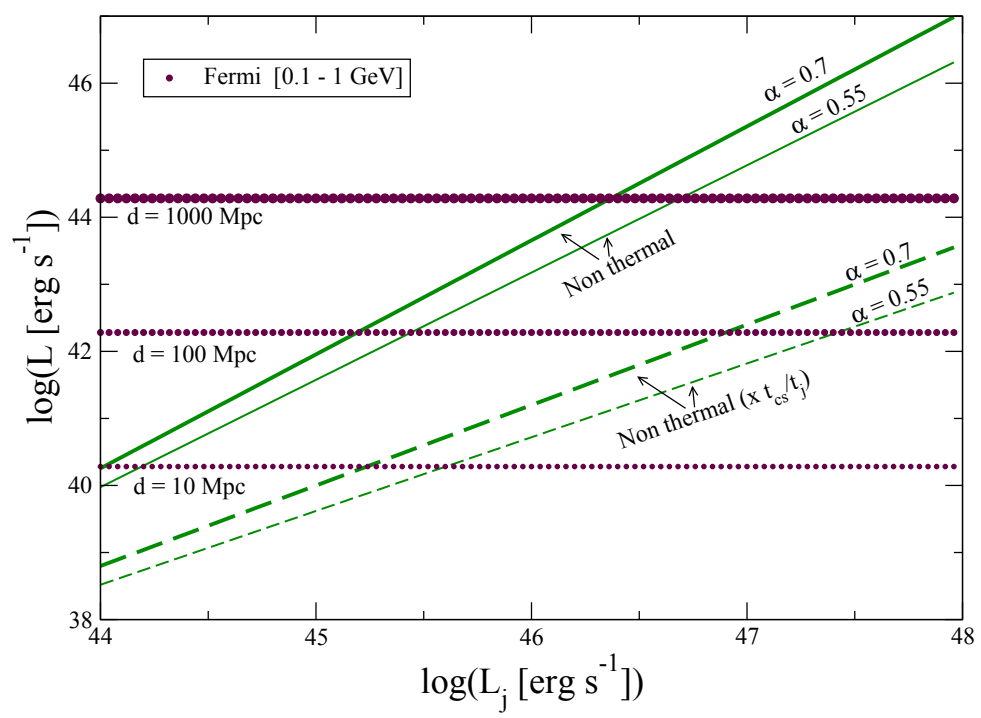

Figura 6.9: Límites superiores para la luminosidad de rayos gamma producida por $N_{\mathrm{n}}^{\mathrm{j}}$ nubes dentro del jet como una función de $L_{\mathrm{j}}$ en fuentes FR II. Dos casos son graficados, uno asumiendo que las nubes cruzan el jet sin ser fragmentadas (líneas sólidas verdes), y otro en en el cual las nubes son destruídas en un tiempo $\sim t_{\mathrm{cn}}$ (líneas rayadas verdes). Las líneas gruesas (sólidas y rayadas) y delgadas (sólidas y rayadas) corresponden a $R_{\mathrm{blr}} \propto L_{\mathrm{blr}}^{0.7}$ (Kaspi et al. 2007) y $R_{\mathrm{blr}} \propto L_{\mathrm{blr}}^{0.55}$ (Kaspi et al. 2005), respectivamente. Además, los niveles de sensibilidad de Fermi en el rango 0.1-1 GeV (líneas punteadas marrones) son graficadas para tres distancias diferentes $d=10,100$ y 1000 Mpc (Araudo et al. 2010). 
Seyfert 2 en el óptico. La masa del agujero negro es $M_{\mathrm{smbh}} \approx 6 \times 10^{7} M_{\odot}$ (Marconi et al. 2000). El ángulo entre los jets y la línea de la visual es grande, $>50^{\circ}$ (Tingay et al. 1998), de tal manera que la radiación producida en el jet no está significativamente corrida en frecuencia por efecto Doppler. Los jets de Cen A son frenados a una distancia $\sim \mathrm{kpc}$, formando dos radio lóbulos gigantes que se extienden $\sim 10^{\circ}$ en el cielo del hemisferio sur. En el óptico, la zona del núcleo de Cen A está oscurecida por una región densa de gas y polvo, formada probablemente en una colición reciente con otra galaxia (Thomson 1992, Mirabel et al. 1999). A energías más altas, los satélites Chandra y XMM-Newton detectaron emisión continua en rayos $\mathrm{X}$ proveniente de la región nuclear, con una luminosidad $\sim 5 \times 10^{41} \mathrm{erg} \mathrm{s}^{-1}$ entre 2-7 keV (Evans et al. 2004). Estos rayos X pueden haber sido producidos en el disco de acreción y en el jet más interno, aunque su origen es aún desconocido. En HE, Cen A ha sido detectada por encima de las $200 \mathrm{MeV}$ por Fermi, con una luminosidad bolométrica de $\sim 4 \times 10^{40} \mathrm{erg} \mathrm{s}^{-1}$ (Abdo et al. 2009b, y por encima de $\sim 200 \mathrm{GeV}$ por HESS, con una luminosidad bolométrica de $\approx 3 \times 10^{39} \mathrm{erg} \mathrm{s}^{-1}$ (Aharonian et al. 2009). En ambos casos, la emisión en HE es asociada a la región nuclear. Cen A ha sido propuesta como una fuente de rayos cósmicos de alta energía por Romero y colaboradores (1996).

Si bien no se han detectado aún líneas de emisión que indiquen la presencia de una BLR en Cen A (Alexander et al. 1999), esto puede ser una consecuencia de que la región nuclear de esta fuente está oscurecida en el óptico por la "estela de polvo". Es por esto que puede haber nubes circundando el SMBH de Cen A (Wang et al. 1986, Risaliti et al. 2002) pero, debido a que el disco de acreción de este AGN es débil, no se espera un nivel alto de fotoionización de estas posibles nubes y por lo tanto el proceso será ineficiente para producir líneas. Bajo esta hipótesis podemos considerar que existe una población de nubes oscuras en la región nuclear de Cen A y aplicar el modelo desarrollado a esta fuente, considerando interacciones EC solamente con los fotones emitidos por el material acretado.

Adoptando $L_{\mathrm{j}}=10^{44} \mathrm{erg} \mathrm{s}^{-1}$ para la luminosidad del jet de Cen A y aquellos valores que han sido listados en la Tabla 6.1 para el resto de los parámetros, $z_{\text {int }}$ resulta $\approx 5 \times 10^{15} \mathrm{~cm}$. A esta altura del jet, la emisión producida por la interacción con una nube es calculada suponiendo que $\eta_{\mathrm{B}}=0.01$. La correspondiente SED se muestra en la Figura 6.10 (izquierda). La parte de más baja energía del espectro sincrotrón es autoabsorbida a energías menores que $\sim 10^{-4} \mathrm{eV}$. En rayos gamma, la absorción gamma-gamma se desprecia debido a que la densidad de fotones ambiente es muy baja (e.g. Rieger \& Aharonian 2009, Araudo et al. 2009, 2010). En HE, la emisión por SSC domina la SED, siendo la luminosidad de este proceso a energías mayores que $100 \mathrm{MeV} \sim 2 \times 10^{39} \mathrm{erg} \mathrm{s}^{-1}$, y por encima de $100 \mathrm{GeV}$ aproximadamente 10 veces menor. Estos valores son un orden de magnitud menores que los correspondientes a los flujos detectados por los telescopios Fermi y HESS. Notamos sin embargo que $L_{e, p} \propto R_{\mathrm{n}}^{2}$, y para nubes apenas más grandes $L_{e, p}$ puede aumentar hasta niveles detectables. La penetración de una nube de tamaño $R_{\mathrm{n}}>10^{13} \mathrm{~cm}$ en el jet de Cen A podría producir un flare de aproximadamente un día de duración. 

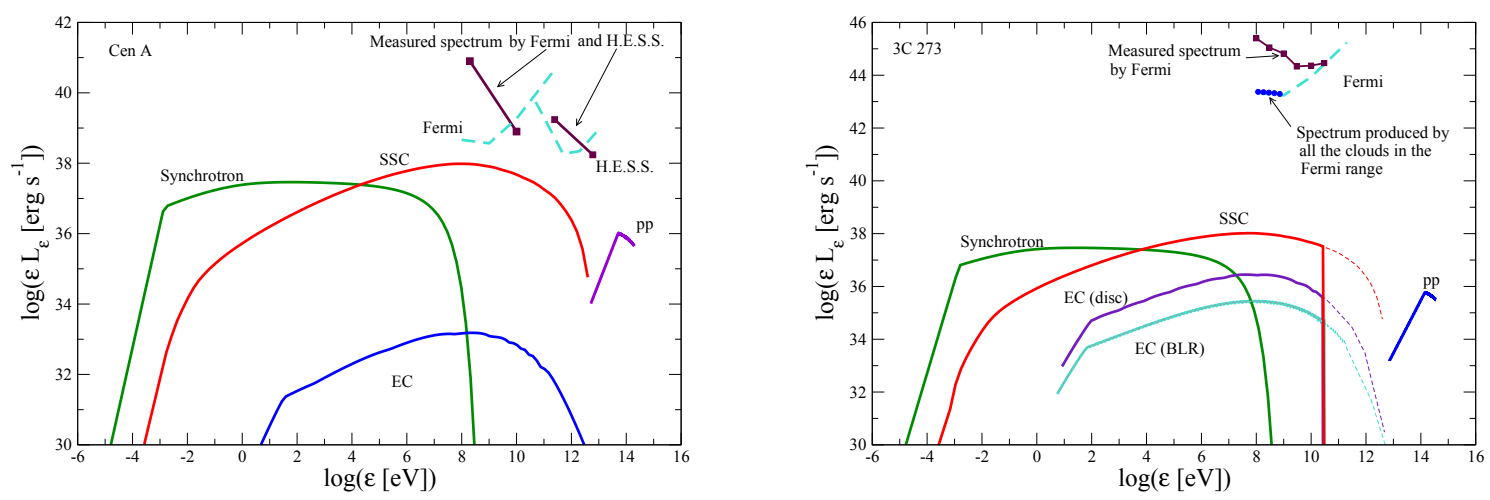

Figura 6.10: Distribuciones espectrales de energía calculadas para las fuentes Cen A (izquierda) y 3C 273 (derecha). En ambos casos se han graficado las luminosidades específicas producidas por los procesos sincrotrón, EC, SSC y $p p$ obtenidos de la interacción de una nube con uno de los jets. También mostramos la emisión detectada por Fermi y HESS y el espectro emitido considerando muchas nubes simultáneamente interactuando con ambos jets en el caso de $3 \mathrm{C} 273$.

Tabla 6.2: Parámetetros adoptados para Cen A y 3C 273.

\begin{tabular}{lcc}
\hline \hline & Cen A & $3 \mathrm{C} 273$ \\
\hline Distancia [Mpc] & 3.7 & $6.7 \times 10^{2}$ \\
Masa del SMBH $\left[M_{\odot}\right]$ & $6 \times 10^{7}$ & $7 \times 10^{9}$ \\
Angulo de inclinación del jet $\left[^{\circ}\right]$ & $>50$ & $\sim 15$ \\
Luminosidad del jet $\left[\mathrm{erg} \mathrm{s}^{-1}\right]$ & $10^{44}$ & $4 \times 10^{47}$ \\
Luminosidad del disco $\left[\mathrm{erg} \mathrm{s}^{-1}\right]$ & $5 \times 10^{41}$ & $2 \times 10^{46}$ \\
Densidad de energía de los fotones del disco $[\mathrm{eV}]$ & $\sim 5 \times 10^{3}$ & 54 \\
Luminosidad de la BLR $\left[\mathrm{erg} \mathrm{s}^{-1}\right]$ & - & $4 \times 10^{45}$ \\
\hline
\end{tabular}




\subsubsection{Galaxias FR II: $3 \mathrm{C} 273$}

A la distancia $d=6.7 \times 10^{2} \mathrm{Mpc}$, 3C 273 es el AGN activo en radio más potente (Courvoisier 1998) con un SMBH de masa $M_{\mathrm{smbh}} \sim 7 \times 10^{9} M_{\odot}$ (Paltani \& Türler 2005). El ángulo entre la dirección del jet con la línea de la visual es chico, $\approx 6^{\circ}$, lo que implica que 3C 273 es un blazar (Jolley et al. 2009). El espectro completo de esta fuente es variable (e.g. Pian et al. 1999) con períodos que van desde años (en radio) hasta unas pocas horas (en rayos gamma). En HE, 3C 273 fue el primer blazar detectedo en la banda $\mathrm{MeV}$ por el satélite COS-B, y luego por EGRET en la banda 0.1 - $10 \mathrm{GeV}$ (Hartman et al. 1999). Esta fuente fue también detectada en energías GeV por Fermi y AGILE, pero aún no ha sido detectada en TeV. Dada la luminosidad del jet de $3 \mathrm{C} 273, L_{\mathrm{j}} \approx 4 \times 10^{47} \mathrm{erg} \mathrm{s}^{-1}$ (Kataoka et al. 2002), $z_{\text {int }}$ resulta $\approx 3 \times 10^{17} \mathrm{~cm}$. La luminosidad de la BLR de esta fuente es $L_{\mathrm{blr}} \approx 4 \times 10^{45} \mathrm{erg} \mathrm{s}^{-1}$ (Cao \& Jiang 1999), y su tamaño $R_{\mathrm{blr}} \sim 7 \times 10^{17} \mathrm{~cm}$ (Ghissellini et al. 2010), lo que implica que las interacciones jet-nube pueden ocurrir por ser $z_{\text {int }}<R_{\mathrm{blr}}$. La luminosidad del disco es alta, $L_{\mathrm{d}} \approx 2 \times 10^{46} \mathrm{erg} \mathrm{s}^{-1}$, con energías típicas de los fotones de $\approx 54 \mathrm{eV}$ (Grandi \& Palumbo 2004).

La SED de la radiación no térmica generada por las interacciones jet-nube en 3C 273 es mostrada en la Figura 6.10 (derecha). A la altura $z_{\text {int }}$, los procesos radiativos más importantes son el sincrotrón y el SSC. Las luminosidades bolométricas generadas por estos procesos en una interacción en $z_{\text {int }}$ son $L_{\mathrm{sin}} \sim 6 \times 10^{38} \mathrm{erg} \mathrm{s}^{-1}$ y $L_{\mathrm{SSC}} \sim 2 \times 10^{39} \mathrm{erg} \mathrm{s}^{-1}$. Dados los fuertes campos de radiación del disco y de la BLR, la emisión por encima de $\sim 10 \mathrm{GeV}$ es absorbida por interacciones fotón-fotón, y el máximo de la emisión ocurre alrededor de $0.1-1 \mathrm{GeV}$. Siendo que el número estimado de nubes en la BLR de 3C 273 es $\sim 10^{8}$ (Dietrich et al. 1999), el factor de llenado que se deduce es $f \sim 3 \times 10^{-7}$ y el número de nubes en ambos jets resulta $\sim 2 \times 10^{3}$ y $5 \times 10^{5}$, para los valores mínimo y máximo dados en la Sección 6.5. En el caso más optimista, la luminosidad SSC podría alcanzar valores $\sim 2 \times 10^{44} \mathrm{erg} \mathrm{s}^{-1}$. Este valor está por debajo de la luminosidad detectada por Fermi en $\mathrm{HE}, \sim 3 \times 10^{46} \mathrm{erg} \mathrm{s}^{-1}$ en el estado estacionario y $\sim 1.7 \times 10^{47} \mathrm{erg} \mathrm{s}^{-1}$ en flares (Soldi et al. 2009). Sin embargo, la emisión detectada probablemente esté muy amplificada por efecto Doppler y esto tapa la emisión que no sufre tal amplificación. Sin embargo, para AGN que no son blazares (galaxias FR II, como Cygnus A), cuya emisión no sufre amplificación Doppler, la radiación en $\mathrm{GeV}$ producida por interacciones jet-nube podrían ser detectadas. En este caso, dado que muchas nubes pueden interactuar simultáneamente con el jet, la emisión será estacionaria.

\subsection{Discusión}

En este capítulo hemos estudiado la interacción de nubes de la BLR con la base de los jets en los AGN. Considerando valores razonables para los parámetros de las nubes y de los jets, estimamos las escalas de tiempo de los procesos dinámicos más relevantes en el escenario analizado, concluyendo que las nubes de la BLR pueden entrar en el jet sólo a $z_{\mathrm{j}} \geq z_{\text {int }}$. Para alturas menores que este valor, el jet es muy compacto y las presiones 
magnética y cinética de éste destruirán a la nube antes de que ésta entre completamente en el jet. Cuando la nube interactúa significativamente con el jet, choques fuertes son generados con la subsecuente emisión en rayos gamma de las partículas aceleradas en tales choques.

Valores del campo magnético en la región chocada del jet $\left(B_{\mathrm{bs}}\right)$ menores que el correspondiente a la equipartición con la energía de las partículas no térmicas permiten una emisión significativa de rayos gamma. Para valores más altos de $B_{\mathrm{bs}}$ (esto es, jets dominados por el flujo de Poynting), el tratamiento desarrollado aquí no es válido. En tal caso, $z_{\text {int }}$ puede aún ser definido si en vez de considerar la presión cinética del jet, consideramos la magnética. Si una nube entra al jet, la aceleración de partículas en el bow shock puede darse por reconeción magnética, por ejemplo. El estudio de este caso requiere de un tratamiento completamente diferente al expuesto en este capítulo. En general, para valores de $B_{\mathrm{bs}}$ mayores que el de equipartición la emisión de rayos gamma por IC es suprimida en favor de la radiación sincrotrón al menos que la disipación magnética reduzca la intensidad del campo magnético lo sufiente como para que el canal de pérdidas IC sea el dominante.

En fuentes cercanas, como por ejemplo Cen A, la interacción de nubes grandes con los jets se debería detectar como un evento esporádico, aunque el número de estas nubes más grandes y el duty cycld 1 de los flares son difíciles de estimar. Dado que los campos de fotones externos son débiles en estas galaxias, fotones de VHE pueden escapar del emisor sin ser absorbidos por interacciones con fotones ambientales. Las interacciones jet-nube en galaxias FR I cercanas serían detectables tanto en HE como en VHE como emisión esporádica con escalas de tiempo de aproximadamente un día. La detección de esta emisión nos proveería de información sobre las condiciones del medio de la región nuclear de los AGN (oscurecida en general en otras frecuencias por la absorción del polvo), como así también de la base de los jets.

En fuentes tipo FR II, muchas nubes pueden interactuar simultáneamente con el jet. El número de éstas $-N_{\mathrm{n}}^{\mathrm{j}}$ - depende fuertemente del tiempo de vida de las nubes dentro del jet, que es del orden de algunas veces $t_{\mathrm{cn}}$. Sin embargo, notamos que aún después de la fragmentación de las nubes, los bow shocks asociados a cada fragmento pueden todavía formarse y acelerar partículas eficientemente si los fragmentos se mueven lentamente, a una velocidad $\ll v_{\mathrm{j}}$. Debido a que las galaxias FR II tienen tasas de acreción altas, la radiación por encima de $1 \mathrm{GeV}$ producida cerca de la base del jet puede ser sustancialmente atenuada por los densos campos de fotones externos (del disco y de la BLR) aunque los rayos gamma de energías $<1 \mathrm{GeV}$ no deberían ser afectados significativamente. Ya que la emisión producida en las interacciones jet-nube es isotrópica, ésta puede ser tapada por la emisión producida en los jets de los blazares y amplificada por efecto Doppler. No obstante, los jets de las galaxias FR II cercanas no muestran un incremento muy importante del flujo y estas fuentes pueden emitir rayos gammas por interacciones jet-nube. En el contexto de la unificación de los AGN (Urry \& Padovani 1995), el número de AGN que no son blazares (intensos en radio) debe ser mucho mayor al de blazares para el mismo valor de

\footnotetext{
${ }^{1}$ Se define el duty cycle de una fuente como la fracción del tiempo de observación durante la cual se producen variaciones significativas de flujo.
} 
$L_{\mathrm{j}}$. Como se muestra en la Figura 6.9 fuentes cercanas e intensas podrían ser detectables por observaciones suficientemente profundas del satélite Fermi. Luego de unos pocos años de exposición, señales significativas de estas fuentes podrían ser encontradas, aportando esto una evidencia de que los jets están dominados por la materia a las alturas de las interacciones con las nubes, además de información sobre las características de la BLR. 


\section{Capítulo 7}

\section{Cúmulos de galaxias}

\subsection{Introducción}

Las galaxias no están aisladas en el Universo sino que se agrupan de a cientos en regiones de un tamaño $\sim 1-10 \mathrm{Mpc}$, donde forman parte de la estructura filamentosa del Universo. Para explicar la formación de estas estructuras a gran escala, el modelo actualmente aceptado contempla la existencia de materia oscura fría (CDM, por Cold Dark Matter). Bajo este paradigma, las galaxias se forman por la fusión de estructuras menores que dan origen a sistemas de mayor escala, como así también por la acreción de material en forma de gas. Durante el proceso de acreción y fusión, la materia se acumula en una red cósmica con sobredensidades moderadas en forma de filamentos, en cuyas intersecciones se ubican las galaxias y los cúmulos de galaxias.

El proceso de acreción contempla la caída de gas difuso proveniente del espacio circundante al cúmulo. Este mecanismo es inestable y anisotrópico, ya que ocurre a través de flujos de material que se propagan a lo largo de los filamentos que convergen en el cúmulo produciendo fuertes choques de acreción al impactar con el medio intra cúmulo (ICM, por Intra Cluster Medium). Por otro lado, la fusión de dos estructuras virializadas puede contemplar la colisión entre dos galaxias, como se cree que ha ocurrido en Cen A, como así también la fusión de dos cúmulos de galaxias. Tanto los procesos de acreción como los de fusión son mucho más intensos en los cúmulos de galaxias que en las galaxias individuales. Esto hace que se produzcan fuertes choques (de acreción y de fusión), los cuales calientan el ICM y aceleran partículas cargadas hasta velocidades relativistas. Tanto la emisión del gas caliente como de las partículas no térmicas caracterizan a los cúmulos de galaxias en diferentes bandas del espectro electromagnético.

En rayos X, los cúmulos de galaxias son los objetos más brillantes del cielo alcanzando una luminosidad $L_{\mathrm{X}} \sim 10^{43}-10^{45} \mathrm{erg} \mathrm{s}^{-1}$. Esta emisión es térmica, producida por el calentamiento del gas intra cúmulo que alcanza temperaturas $\sim 10^{8} \mathrm{~K}$. Sin embargo, la detección de estas estructuras en frecuencias radio como así también en el extremo UV $(0.07-0.4 \mathrm{keV})$ y en los rayos $\mathrm{X}$ duros $(20-80 \mathrm{keV})$ indica la presencia de actividad no térmica en estos sitemas. La emisión en frecuencias radio detectada en los cúmulos de 


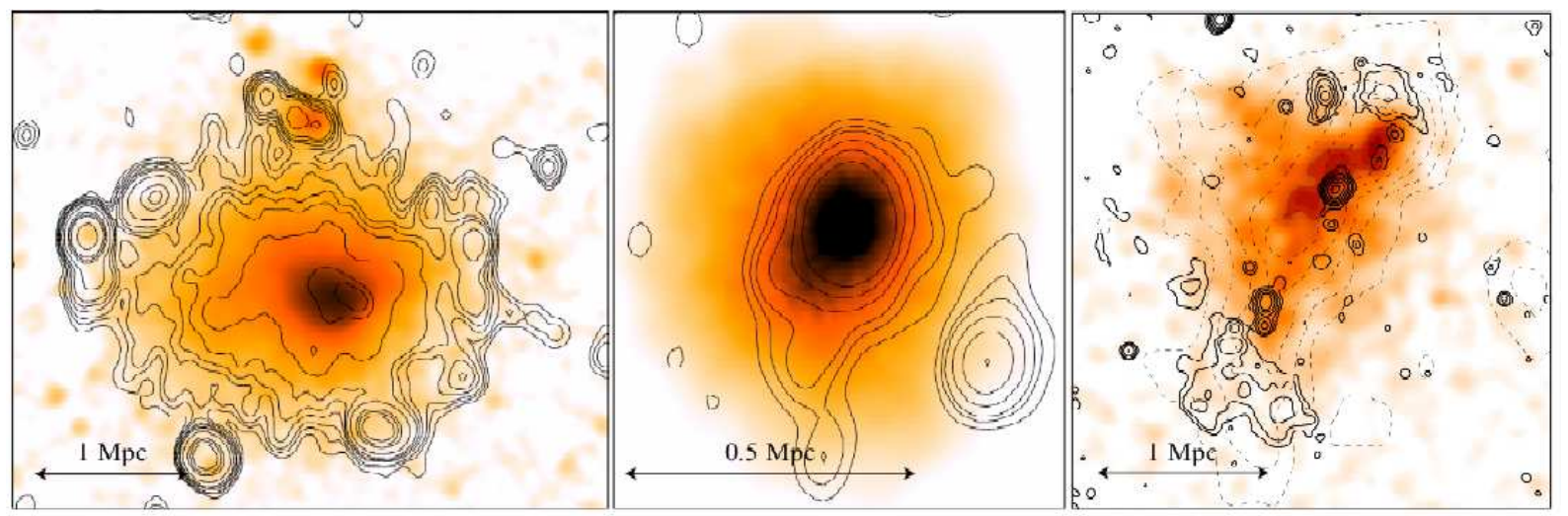

Figura 7.1: Los cúmulos de galaxias Abell 2163 (izquierda), RX J1347.5-1145 (medio) y ZwCl 2341.1 (derecha) observados en rayos X (amarillo-anaranjado-marrón) y en frecuencias radio (contornos de líneas llenas) son mostrados. Uno de los halos más luminosos que se han detectado es aquel asociado al cúmulo A2163. En el centro de la fuente RX J1347.51145 , la más luminosa en rayos $\mathrm{X}$, se ha detectado un halo pequeño, mientras que remanentes dobles han sido detectados en los bordes del cúmulo $\mathrm{ZwCl}_{w} 2341.1$ (Ferrari 2010).

galaxias es originada en la interacción de electrones relativistas con el campo magnético del cúmulo $(\sim \mu \mathrm{G})$. Por otro lado, la radiación en el extremo UV debe ser producida o bien por la componente térmica más fría o bien por interacciones IC con los fotones del fondo cósmico de radiación (CMB, por Cosmic Microwave Background) con la misma población de electrones que produce la emisión sincrotrón en radio. La radiación sincrotrón también podría explicar la emisión de los rayos X duros, aunque esto es todavía tema de debate.

Respecto de la emisión en radio, aunque su naturaleza no térmica está bien establecida por las observaciones, ésta puede ser debida a dos tipos de fuentes diferentes: halos o remanentes (relics). Los primeros están localizados en los centros de los cúmulos y la emisión en radio de éstos no está polarizada y se superpone a la emisión térmica en rayos X. Los últimos se encuentran en la periferia de los cúmulos y son regiones de emisión polarizada y con una estrucura más irregular. El modelo actualmente aceptado para la formación de estos halos y remanentes es que se producen por choques de acreción y de fusión, respectivamente. En la Figura 7.1 se muestran estos dos tipos de fuentes.

Los remanentes han sido observados en muchos cúmulos, aunque los más poderosos y extendidos han sido detectados en fuentes con halos centrales, como el cúmulo Coma (Giovannini et al. 1991) y las fuentes Abell 2163 (Feretti et al. 2001), Abell 2255 (Feretti et al. 1997), Abell 2256 (Rottgering et al. 1994) y Abell 2744 (Govoni et al. 2001). Sólo muy pocos cúmulos presentan dos remanentes (opuestos el uno al otro), siendo los ejemplos más prominentes aquellos encontrados en los cúmulos Abell 3667 (Rottgering et al. 1997) y Abell 3376 (Bagchi et al. 2006).

Dado que hay una población de partículas relativistas que emiten la radiación sincrotrón y quizas la emisión de rayos $\mathrm{X}$ duros por IC, estas mismas partículas pueden producir 
también emisión de más alta energía. La radiación gamma sería generada por interacciones $p p$ con el ICM si protones son acelerados (ver Völk et al. 1996), o por dispersiones IC entre los fotones del CMB y los electrones relativistas (Atoyan \& Völk, 2000). Las simulaciones cosmológicas hidrodinámicas son útiles para predecir los niveles de emisión de rayos gamma en objetos virializados a gran escala (Keshet et al. 2003; Pfrommer et al. 2007, 2008). Si embargo, los resultados de estos estudios no predicen una contribución significativa al fondo difuso de rayos gamma (Berrington \& Dermer 2003). Estos resultados teóricos son consistentes con el hecho de que no se hayan detectado cúmulos de galaxias en HE y VHE con los telescopios de rayos gamma que actualmente entán funcionando. Sólo han sido reportadas evidencias marginales de emisión de altas energías del cúmulo Abell 1758 dentro de la caja de error de la fuente 3 EG J1337+5029 (Fegan et al. 2005). Los cúmulos de galaxias Perseus y Abell 2029 fueron observados por Perkins y colaboradores (2006) con el telescopio Cherenkov Whipple, aunque no encontraron evidencias de fuentes de rayos gamma puntuales ni extendidas en el rango de los TeV. Por otro lado, observaciones recientes de los cúmulos Coma y Abell 496 llavadas a cabo con HESS (Domainko et al 2007) no detectaron emisión significativa en rayos gamma a partir de observaciones con tiempos de exposición moderados, de $\sim 10-20$ horas.

Todas las estimaciones hechas al momento de la posible emisión de rayos gamma de los cúmulos de galaxias han sido realizadas para fuentes que no son reales sino simuladas numéricamente. Por esto, en el presente capítulo estudiaremos los procesos no térmicos que tienen lugar en los bordes del cúmulo Abell 3376, en donde se han detectado en frecuencias radio dos remanentes gigantes y simétricos. En lo que sigue, analizaremos tanto el contenido de partículas relativistas en los remanentes de este cúmulo como así también la emisión no térmica producida por las partículas aceleradas, concentrandonós en el análisis del espectro de rayos gamma.

\section{2. $\quad$ El cúmulo Abell 3376}

El cúmulo de galaxias Abell 3376 está ubicado a una distancia $d \sim 187 \mathrm{Mpc}$, con un corrimiento al rojo (redshift) $z \sim 0.046$ si consideramos una cosmología estándar de materia oscura fría $\Lambda \mathrm{CDM}\left(\Omega_{\mathrm{m}}=0.3, \Omega_{\Lambda}=0.7, \mathrm{H}_{\mathrm{o}}=100 h^{-1} \mathrm{~km} \mathrm{~s}^{-1} \mathrm{Mpc}^{-1}\right.$, con $\left.h=0.7\right)$. La masa del cúmulo ha sido estimada aplicando el teorema del virial a las galaxias miembro del mismo. Asumiendo que la distribución de masa de la fuente se comporta de la misma manera que la distribución de masa de las galaxias miembro (Girardi et al. 1998), obtenemos una masa virial $M_{\mathrm{A} 3376} \sim 3.64 \times 10^{14} h^{-1} M_{\odot}$. El correspondiente radio virial resulta $R_{\mathrm{A} 3376} \sim 1.4 h^{-1} \mathrm{Mpc}$.

El cúmulo Abell 3376 ha sido detectado por los satélites ROSAT y XMM-Newton en rayos $\mathrm{X}$ siendo esto una evidencia de la actividad de fusión de subcúmulos. La luminosidad en $\mathrm{X}$ de esta fuente es $L_{\mathrm{X}}(0.1-2.4 \mathrm{keV}) \simeq 2.48 \times 10^{44} \mathrm{erg} \mathrm{s}^{-1}$, producida térmicamente por el ICM caliente, que tiene una temperatura promedio $T_{\mathrm{X}} \approx 5.8 \times 10^{7} \mathrm{~K}(\approx 5 \mathrm{keV})$.

Observaciones en radio llevadas a cabo por Bagchi y colaboradores (2006) con el arreglo de telescopios VLA muestran la presencia de dos estructuras gigantes en el borde del 


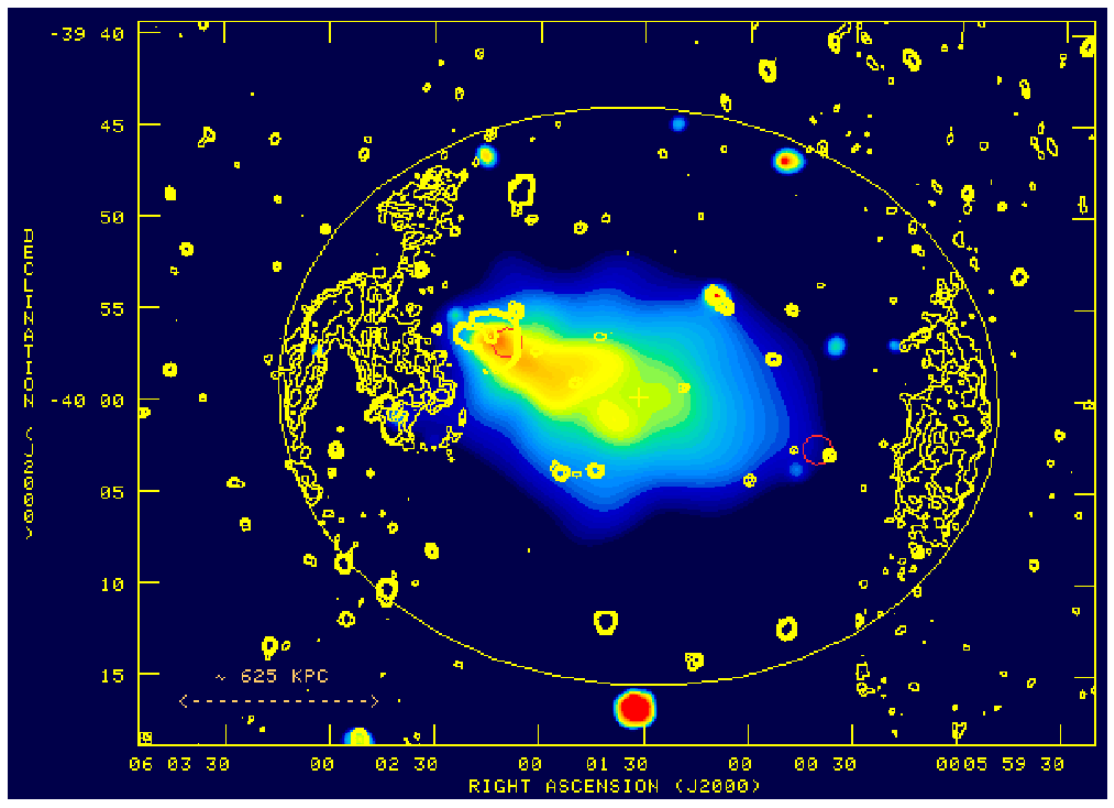

Figura 7.2: Composición de la emisión en frecuencias radio (contornos amarillos) y en rayos X (estructura central) del cúmulo Abell 3376. Con amarillo se dibuja una elipse dentro de la cual están la emisión de rayos X (en el centro) y los remanentes (en el borde) (Bagchi et al. 2006). 

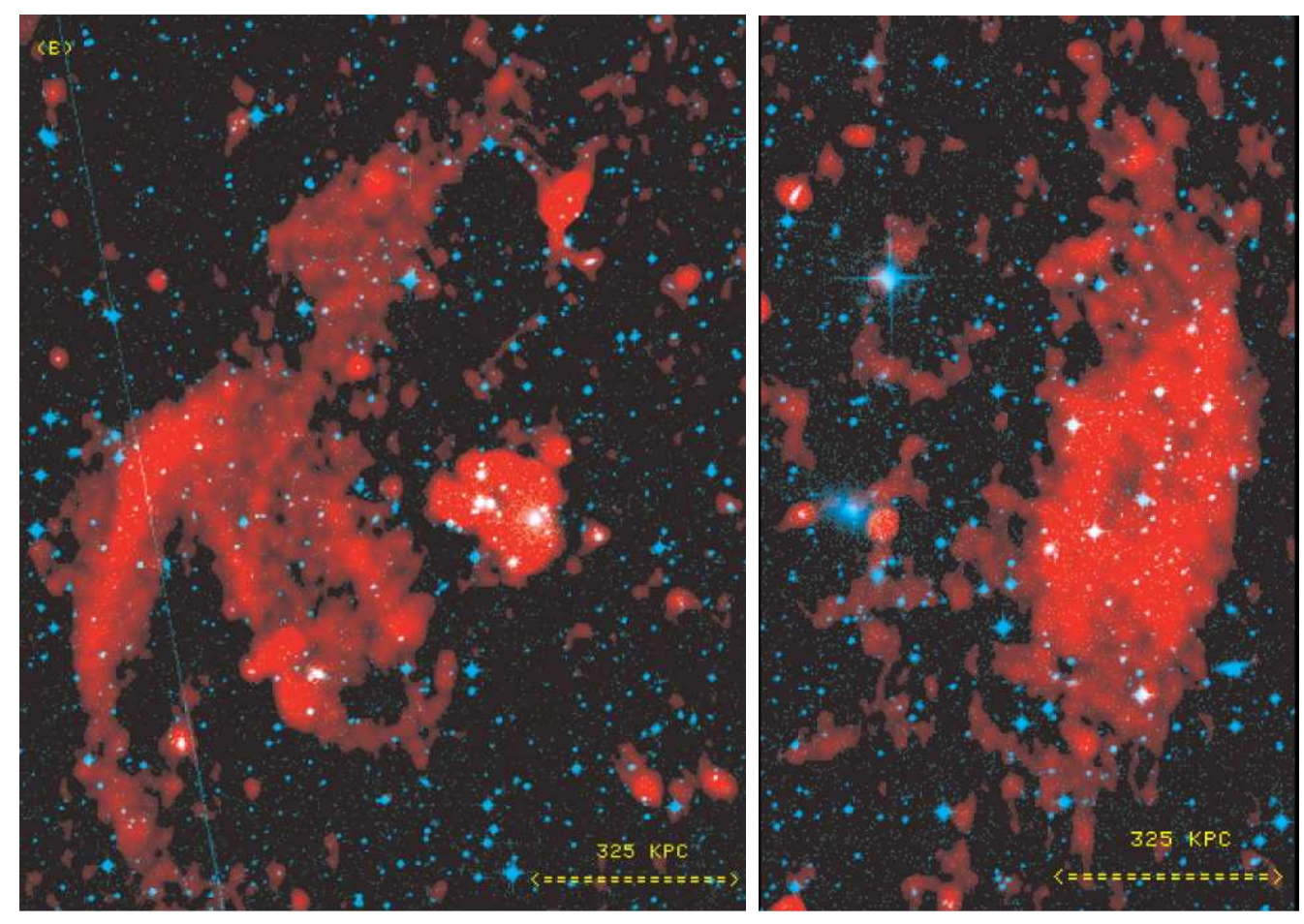

Figura 7.3: Mapas compuestos en frecuencias radio y ópticas de los remanentes este (izquierda) y oeste (derecha) del cúmulo Abell 3376. En rojo se muestra la emisión en radio y en azul la radiación detectada en óptico (Bagchi et al. 2006). 
cúmulo, a una distancia $\sim 1 \mathrm{Mpc}$ del centro. El flujo detectado en radio a $\nu=1.4 \mathrm{GHz}$ es $S_{\nu}=302 \mathrm{mJy}$, que corresponde a una luminosidad de $2.1 \times 10^{40} \mathrm{erg} \mathrm{s}^{-1}$. Estas estructuras tienen las características típicas de los radio remanentes (relics) (Giovannini \& Ferreti 2004). Ellas se ajustan bien con una elipse proyectada en el plano del cielo de semiejes menor y mayor $\sim 1.6 \mathrm{y} \sim 2 \mathrm{Mpc}$, respectivamente, como se muestra en la Figura 7.2 Adoptando una profundidad de la línea de la visual en la elipse de $\sim 270 \mathrm{kpc}$ (Bagchi et al. 2006), el volumen del elipsoide tridimensional resulta $\sim 0.45 \mathrm{Mpc}^{3}$. De la figura mencionada anteriormente inferimos que sólo el $20 \%$ de este volumen corresponde a los remanentes en radio, es decir, $V_{\mathrm{r}} \sim 0.09 \mathrm{Mpc}^{3}$. En la Figura 7.3 se muestra con más detalle la estructura y tamaño de cada uno de los remanentes.

Algunos parámetros del cúmulo que son relevantes para los propósitos de nuestro estudio, como la densidad del gas en los remanentes, $n_{\mathrm{r}}$, y la velocidad del choque, $v_{\mathrm{ch}}$, no son provistos por las observaciones y para hallarlos nos valemos de simulaciones numéricas. Considerando una simulación hidrodinámica de $N$-cuerpos/SPH (Smoothed Particle Hydrodynamics ) para un cúmulo cuya masa virial es $M_{\text {vir }} \sim 1.3 \times 10^{15} h^{-1} M_{\odot}\left(\sim M_{\mathrm{A} 3376}\right)$, del análisis de los resultados de esta simulación obtenemos que $n_{\mathrm{r}}=2 \times 10^{-5} \mathrm{~cm}^{-3} \mathrm{y}$ $v_{\mathrm{ch}}=1000 \mathrm{~km} \mathrm{~s}^{-1}$. Teniendo en cuenta que la temperatura del ICM, donde el choque se está propagando, es $T_{\mathrm{ICM}} \sim 0.1 T_{\mathrm{r}}$ (Hoeft et al. 2004) el número de Mach resulta $M \sim 4.2$ (Gabici \& Blasi 2003), donde $T_{\mathrm{r}}$ es la temperatura del ICM en la región de los remanentes. En el resto del capítulo, expresaremos los resultados numéricos adoptando $h=0.7$. En la Tabla 7.1 listamos los valores de los parámetros (observados y estimados) de la fuente Abell 3376.

\begin{tabular}{ll}
\hline \hline Parámetro & valor \\
\hline Corrimiento al rojo & $z=0.046$ \\
Distancia & $d=187 \mathrm{Mpc}$ \\
Masa & $M_{\mathrm{A} 3376} \sim 5.2 \times 10^{14} M_{\odot}$ \\
Tamaño & $R_{\mathrm{A} 3376} \sim 2 \mathrm{Mpc}$ \\
Volumen de los remanentes & $V_{\mathrm{r}} \sim 0.09 \mathrm{Mpc}^{3}$ \\
Densidad & $n_{\mathrm{r}}=2 \times 10^{-5} \mathrm{~cm}^{-3}$ \\
Velocidad de los choques & $v_{\mathrm{ch}}=1000 \mathrm{~km} \mathrm{~s}^{-1}$ \\
Luminosidad en X & $L_{\mathrm{X}}=2.48 \times 10^{44} \mathrm{erg} \mathrm{s}^{-1}$ \\
Luminosidad en radio & $2.1 \times 10^{40} \mathrm{erg} \mathrm{s}^{-1}$ \\
Temperatura del gas & $T_{\mathrm{X}} \approx 5.8 \times 10^{7} \mathrm{~K}$ \\
\hline
\end{tabular}

Tabla 7.1: Parámetros del cúmulo de galaxias Abell 3376. Los valores están dados considerando $h=0.7$. 

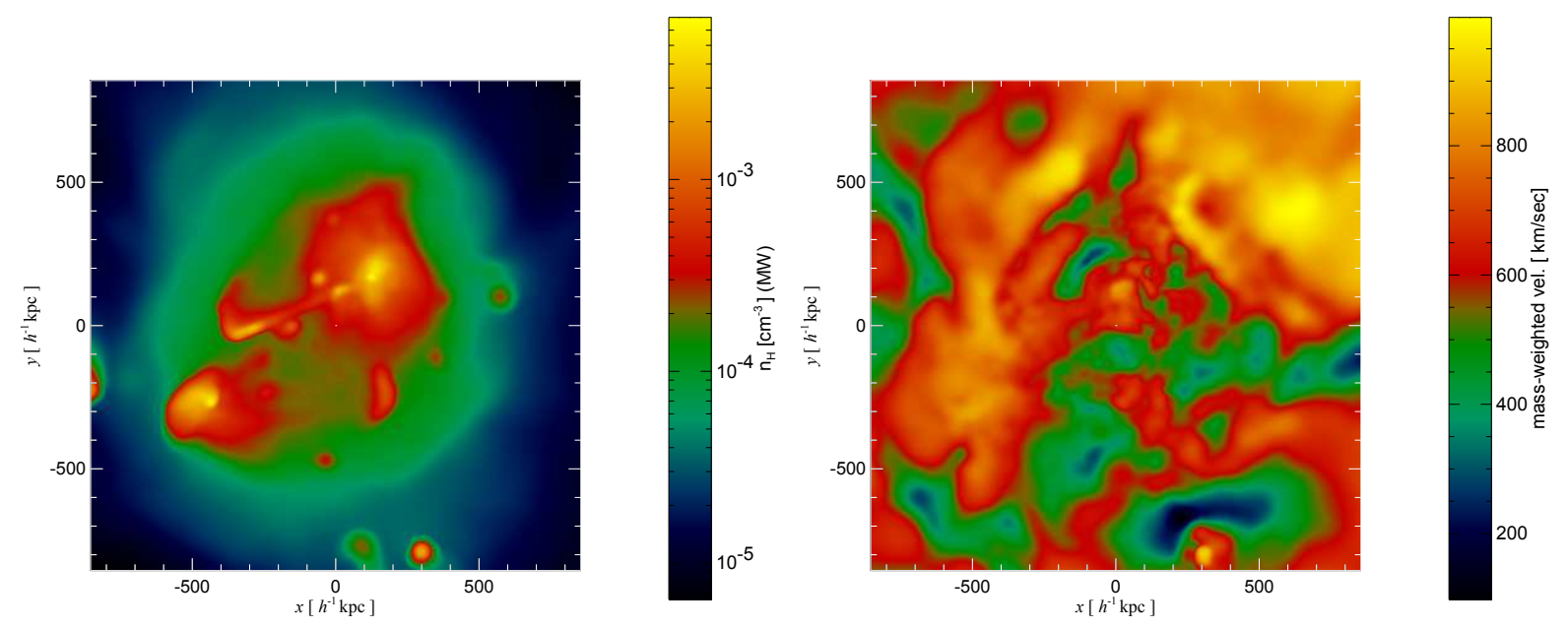

Figura 7.4: Mapas de densidad (izquierda) y de velocidad (derecha) de una simulación de un cúmulo de galaxias cuya masa virial es $\sim 1.3 \times 10^{15} h^{-1} M_{\odot}$. Del análisis de estas figuras podemos obtener una estimación de los valores de la densidad y de la velocidad de los choques a una distancia $\sim 1 \mathrm{Mpc}$ del centro (donde se localizan los remanentes en la fuente Abell 3376). Hallamos $n_{\mathrm{r}} \sim 2 \times 10^{-5} \mathrm{~cm}^{-3}$ y $v_{\mathrm{ch}} \sim 1000 \mathrm{~km} \mathrm{~s}^{-1}$.

\subsection{Población de partículas relativistas}

Las ondas de choque que se generan (por acreción y fusión) durante la formación y evolución de los cúmulos de galaxias son la fuente principal de la termalización del ICM y de la aceleración de partículas (Pfrommer et al. 2006). La actividad de las radiogalaxias inmersas en los cúmulos también contribuye a la población de partículas relativistas, inyectando un plasma fósil que se detecta como cavidades en los mapas de brillo superficial de rayos X (Churazov et al. 2000). En el cúmulo Abell 3376 se han detectado dos radiogalaxias. Una de ellas es la fuente MRC 0600-399, asociada con el segundo miembro más luminoso del cúmulo, y la otra radiofuente es originada posiblemente por una galaxia elíptica. Estas dos radiogalaxias se encuentran dentro de la región central del cúmulo, donde la emisión térmica es detectada. Por esto, consideramos que las estructuras que se detectan en frecuencias radio en el borde del cúmulo no están asociadas a estas radiogalaxias (que son fuentes puntuales).

La morfología de la emisión en frecuencias radio y en rayos $\mathrm{X}$ observada en el cúmulo Abell 3376 sugiere que éste está sufriendo una fusión con otro cúmulo de masa similar. Como muestran los cálculos numéricos que simulan la fusión de dos cúmulos de galaxias (Hoeft et al. 2004), las ondas de choque se propagan en ambas direcciones a lo largo de la línea que conecta los centros de ambos cúmulos con los remanentes casi exclusivamente observados en la localización de los frentes de choque. En los casos en los cuales sólo se detecta un único remanente, probablemente la fusión haya ocurrido entre dos cúmulos de 


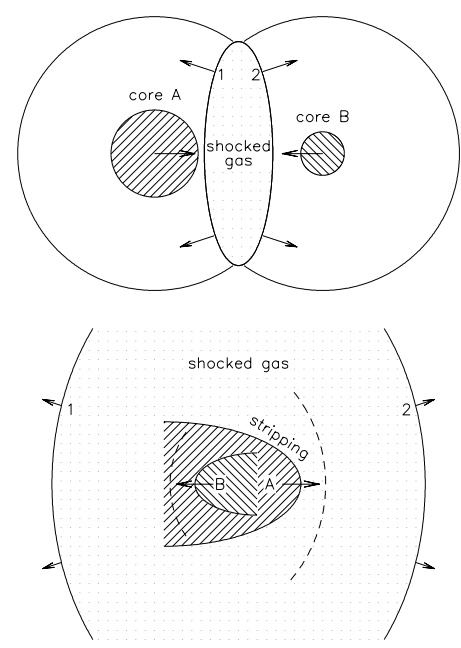

Figura 7.5: Esquema de la fusión de dos cúmulos (cores). En la figura de arriba se muestra el sistema antes de la fusión y en la de abajo después. Como puede observarse en el último gráfico, luego de la colisión queda un núcleo compuesto por la fusión de ambos cúmulos y dos choques que se propagan en direcciones opuestas y hacia afuera del sistema (Markevitch et al. 2000).

masas muy diferentes y sólo el choque que se propaga a través del cumulo más masivo perdura en el tiempo y se detecta. El tiempo de vida de estas estructuras suele estimarse como $\tau_{\text {vida }} \sim R_{\mathrm{r}} / v_{\mathrm{ch}}$, donde $R_{\mathrm{r}}$ es la distancia recorrida por los choques. En el caso de la fuente que estamos estudiando, $R_{\mathrm{r}} \sim 1 \mathrm{Mpc}$ y $v_{\mathrm{ch}} \sim 1000 \mathrm{~km} \mathrm{~s}^{-1}$, con lo cual $\tau_{\text {vida }}$ resulta $\sim 1$ Gyr. Notamos que estos choques no llegan a un estado estacionario ya que son el producto de un evento puntual en el tiempo: una fusión. La energía que llevan los choques de fusión se disipa a medida que éstos se propagan por el ICM.

Teniendo en cuenta las evidencias observacionales y los resultados numéricos, suponemos que el contenido de partículas relativistas en los remanentes es debido a la aceleración en los choques de fusión, despreciando la posible contribución de las radiogalaxias y de los choques de acreción. En las siguientes subsecciones describiremos los procesos de aceleración y enfriamiento tanto de electrones (de los cuales tenemos evidencia observacional) como de protones, que determinan las distribuciones de energía de estas partículas y su subsiguiente evolución.

\subsubsection{Aceleración de partículas y pérdidas radiativas}

La emisión difusa en radio producida en la localización de los remanentes es evidencia de la presencia de electrones relativistas en esa región. Por otro lado, aunque no tengamos evidencia observacional de la presencia de protones en los remanentes, estas partículas pueden ser aceleradas también de la misma manera que los electrones. Para estimar el tiempo en el cual estas partículas se aceleran hasta una energía dada necesitamos conocer 
Tabla 7.2: Campo magnético, $B_{\mathrm{r}}$, y constantes de normalización de la distribución de electrones primarios, $k_{e_{1}}^{\prime}$, y protones, $k_{p}^{\prime}$, acelerados en los remanentes para los tres casos considerados y caracterizados por el parámetro $a$.

\begin{tabular}{lccc}
\hline \hline$a$ & $\begin{array}{c}B_{\mathrm{r}} \\
{[\mathrm{G}]}\end{array}$ & $\begin{array}{c}k_{e_{1}}^{\prime} \\
{\left[\mathrm{erg}^{p_{e_{1}}-1} \mathrm{~cm}^{-3}\right]}\end{array}$ & $\begin{array}{c}k_{p}^{\prime} \\
{\left[\mathrm{erg}^{p_{p}-1} \mathrm{~cm}^{-3}\right]}\end{array}$ \\
\hline 0 & $9 \times 10^{-7}$ & $1.4 \times 10^{-15}$ & - \\
1 & $1.1 \times 10^{-6}$ & $1.1 \times 10^{-15}$ & $8.7 \times 10^{-16}$ \\
100 & $3.4 \times 10^{-6}$ & $3 \times 10^{-16}$ & $2.5 \times 10^{-14}$ \\
\hline
\end{tabular}

la magnitud del campo magnético $B_{\mathrm{r}}$ en los remanentes/acelerador.

Para estimar $B_{\mathrm{r}}$ suponemos que la energía se reparte de la misma manera tanto en el campo magnético, $u_{\mathrm{B}}$, como en las partículas relativistas, $u_{\mathrm{nt}}$ :

$$
\frac{B_{\mathrm{r}}^{2}}{8 \pi}=u_{\mathrm{nt}}=u_{e_{1}}+u_{p}+u_{e_{2}},
$$

donde $u_{e_{1}}$ y $u_{p}$ son las densidades de energía de los electrones y protones primarios, respectivamente, y $u_{e_{2}}$ la densidad de energía de los pares secundarios. De la misma manera que en el Capítulo 4. para estimar $u_{\mathrm{B}}$ a partir de (7.1) necesitamos conocer las distribuciones de partículas, es decir, las constantes de normalización $k_{i}^{\prime}$, para $i=e_{1}, p, e_{2}$, y los índices $p_{i}^{\prime}$ de las potencias $\left(n_{i}\left(E_{i}\right)=k_{i}^{\prime} E_{i}^{-p_{i}^{\prime}}\right)$. Para esto procedemos de la misma manera que se ha expuesto en la Sección 4.3.2, fijando $u_{p}=a u_{e_{1}}$ y considerando tres valores para el parámetro libre $a: 0,1$ y 100; además de tener en cuenta que $u_{e_{2}}=f_{e^{ \pm}} u_{p}$.

Para que la producción de pares secundarios sea eficiente, es necesario un medio con una densidad alta. Sin embargo, esta condición no se satisface en las regiones periféricas de los cúmulos, las cuales están caracterizadas por densidades muy bajas del gas $\left(\sim 10^{-5} \mathrm{~cm}^{-3}\right)$. Por esto, la contribución de $u_{e_{2}}$ en la ecuación (7.1) es despreciable y no la tenemos en cuenta en los cálculos de $B_{\mathrm{r}}$. Luego, aplicando la restricción de que el flujo observado en radio de la fuente Abell 3376 es $S(\nu=1.4 \mathrm{GHz})=302 \mathrm{mJy}$, podemos estimar el valor de $B_{\mathrm{r}}$ y de las constantes $k_{i}^{\prime}$ asumiendo que $p_{e_{1}}^{\prime}=p_{p}^{\prime}=2.1$. Los resultados obtenidos se muestran en la Tabla 7.2. Considerando estos valores obtenemos que $p_{e_{2}}=2.08$ y el cociente entre las densidades de energía de los pares $e^{ \pm}$y de los protones es muy chico, $u_{e_{2}} / u_{p} \sim 10^{-5}$, lo que justifica nuestra suposición previa sobre la contribución de los pares secundarios al flujo detectado en frecuencias radio.

Asumiendo que la velocidad del choque es $v_{\mathrm{ch}}=1000 \mathrm{~km} \mathrm{~s}^{-1}$, el tiempo de aceleración resulta

$$
t_{\mathrm{ac}} \sim 0.5\left(\frac{B_{\mathrm{r}}}{1 \mu \mathrm{G}}\right)^{-1}\left(\frac{E_{e}}{1 \mathrm{erg}}\right) \mathrm{s} .
$$

Las pérdidas por escape no son relevantes ya que las escalas espaciales que deben recorrer las partículas para escapar de los remanentes son muy grandes. Siendo el ancho del remanente 
$\sim 2 l_{\mathrm{r}} \sim 0.6 \mathrm{Mpc}($ ver la Figura 7.3 ) tenemos que el tiempo de convección a una velocidad $\sim v_{\mathrm{ch}} / 4$ es muy largo,

$$
t_{\text {conv }} \sim \frac{2 l_{\mathrm{r}}}{v_{\mathrm{ch}} / 4} \sim 7 \times 10^{16} \mathrm{~s} .
$$

Para estimar el tiempo de difusión consideramos que la escala espacial mínima que deben recorrer las partículas para difundir y escapar del remanente es $l_{\mathrm{r}} \sim 0.3 \mathrm{Mpc}$ y hallamos que

$$
t_{\text {dif }} \sim 2 \times 10^{22}\left(\frac{B_{\mathrm{r}}}{1 \mu \mathrm{G}}\right)\left(\frac{E_{i}}{1 \mathrm{erg}}\right)^{-1} \mathrm{~s} .
$$

\section{Pérdidas leptónicas}

Las pérdidas radiativas más importantes que sufrirán los electrones en los remanentes de fusiones son por radiación sincrotrón, por Bremsstrahlung relativista y por dispersiones IC. Las primeras están caracterizadas por un tiempo de enfriamiento (ver (3.15))

$$
t_{\mathrm{sin}} \sim 4.1 \times 10^{14}\left(\frac{B_{\mathrm{r}}}{\mu \mathrm{G}}\right)^{-2}\left(\frac{E_{e_{1}, p}}{1 \mathrm{erg}}\right)^{-1} \mathrm{~s} .
$$

Para las pérdidas por IC consideramos el campo de fotones provistos por el CMB como así también los rayos X emitidos térmicamente por el ICM caliente. En el primer caso, la densidad de energía del CMB es $u_{\mathrm{CMB}}=1.2 \times 10^{-13} \mathrm{erg} \mathrm{cm}^{-3}$ al redshift $z$ correspondiente al cúmulo Abell 3376. En el último caso $u_{\mathrm{X}}=L_{\mathrm{X}}\left(4 \pi R_{\mathrm{X}}^{2} c\right)^{-1}(1+z)^{4}$, donde $R_{\mathrm{X}}$ es el radio de la región emisora de los fotones $\mathrm{X}$ y es $\sim 0.15-0.3 R_{\mathrm{vir}}$ (Balestra 2007), con lo cual en la fuente que estamos estudiando resulta $R_{\mathrm{X}} \approx 0.3 \mathrm{Mpc}$, dando $u_{\mathrm{X}} \approx 1.3 \times 10^{-15} \mathrm{erg} \mathrm{cm}^{-3}$. Debido a que $u_{\mathrm{X}} \ll u_{\mathrm{CMB}}$ sólo consideraremos los fotones del CMB para estimar las pérdidas por IC. Teniendo en cuenta que la energía característica de los fotones del CMB es $E_{\mathrm{ph}}^{\mathrm{CMB}} \sim 1.9 \times 10^{-4} \mathrm{eV}$, sólo aquellos electrones con energías mayores que $1.6 \times 10^{15} \mathrm{eV}$ van a interactuar en el régimen de $\mathrm{KN}$. El tiempo de enfriamiento por IC con los fotones del CMB resulta

$$
t_{\mathrm{IC}}=15.25 \frac{(1+8.3 y)}{\ln (1+0.2 y)} \frac{\left(1+1.3 y^{2}\right)}{\left(1+0.5 y+1.3 y^{2}\right)} \mathrm{s},
$$

donde $y \equiv E_{\mathrm{ph}}^{\mathrm{CMB}} E_{e} /\left(5.1 \times 10^{5} \mathrm{eV}\right)^{2}$.

Finalmente, considerando que la densidad del medio en la localización de los remanentes es $n_{\mathrm{r}}=2 \times 10^{-5} \mathrm{~cm}^{-3}$, el tiempo de enfriamiento por Bremsstrahlung relativista es muy grande, $t_{\text {Brem }} \sim 2 \times 10^{22} \mathrm{~s}$, con lo cual este proceso no será relevante. En la Figura 7.6 se muestran los tiempos de enfriamiento calculados anteriormente juntamente con los tiempos de aceleración y de escape (convección) para el caso $a=1$. Como puede observarse, la energía máxima de los electrones primarios queda determinada por la igualdad $t_{\mathrm{ac}}=t_{\mathrm{IC}} \mathrm{y}$ da un valor $E_{e_{1}}^{\max } \sim 9 \times 10^{13} \mathrm{eV}$ para diferentes valores de $a$, como se muetra en la segunda columna de la Tabla 7.3. Para los electrones con energías $\sim E_{e_{1}}^{\max }$, encontramos que el radio de giro de estas partículas es $r_{\mathrm{g}} \sim 0.1 \mathrm{pc}\left(\ll l_{\mathrm{r}}\right)$, con lo cual están contenidas dentro de la región de aceleración. 


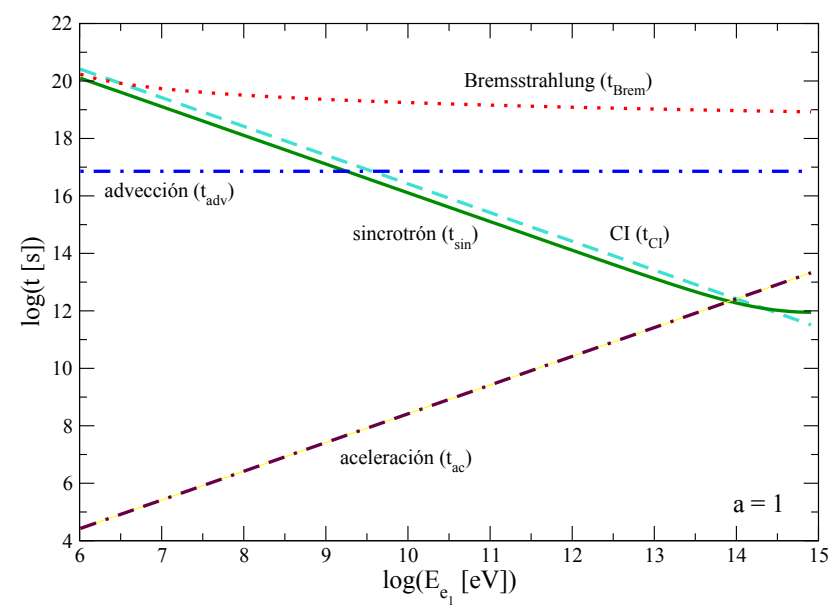

Figura 7.6: Tiempos de aceleración y de enfriamiento para los electrones primarios. Esta figura corresponde al caso con $a=1$. Al resto de los casos les corresponden gráficos similares, ya que la única diferencia está en el valor de $B_{\mathrm{r}}$ (que afecta a $t_{\mathrm{ac}}, t_{\mathrm{sin}}$ y $t_{\mathrm{dif}}$ ) y éste no cambia demasiado ( $B_{\mathrm{r}} \sim 10^{-6} \mathrm{G}$ para los 3 valores de $a$ considerados).

Para estimar el estado evolutivo de la población de electrones relativistas acelerados e inyectados en los remanentes, consideramos la ecuación (2.64) con un tiempo de vida de la fuente $\tau_{\text {vida }} \sim 1$ Gyr. Los resultados se muestran en la Figura 7.7para los casos $a=1$ y 100 y para diferentes tiempos de inyección $t$. Como se muestra en estos gráficos, los espectros presentan un quiebre en $E_{\mathrm{q}} \sim 5$ y $1 \mathrm{GeV}$, para los casos $a=1$ y 100 , respectivamente. Este quiebre se debe a que para $E_{e_{1}}<E_{\mathrm{q}}$, las pérdidas de energía por escape son más eficientes que el enfriamiento radiativo. Finalmente, dado que $t_{\text {conv }} \sim \tau_{\text {vida }}$, podemos considerar que el espectro de los electrones relativistas inyectados en los remanentes alcanza el estado presente después de un tiempo $\sim \tau_{\text {vida }}$.

Tabla 7.3: Las energías máximas obtenidas para los electrones primarios, $E_{e_{1}}^{\max }$, y protones, $E_{p}^{\max }$, acelerados en los remanentes de los choques de fusión del cúmulo Abell 3376. Las energias máximas $E_{e_{2}}^{\max }$ alcanzadas por los pares $e^{ \pm}$son también presentadas.

\begin{tabular}{cccc}
\hline \hline $\mathrm{a}$ & $\begin{array}{c}E_{e_{1}}^{\max } \\
{[\mathrm{eV}]}\end{array}$ & $\begin{array}{c}E_{p}^{\max } \\
{[\mathrm{eV}]}\end{array}$ & $\begin{array}{c}E_{e_{2}}^{\max } \\
{[\mathrm{eV}]}\end{array}$ \\
\hline 0 & $9 \times 10^{13}$ & - & - \\
1 & $9.3 \times 10^{13}$ & $5.0 \times 10^{17}$ & $4 \times 10^{16}$ \\
100 & $8.8 \times 10^{13}$ & $1.3 \times 10^{18}$ & $10^{17}$ \\
\hline
\end{tabular}



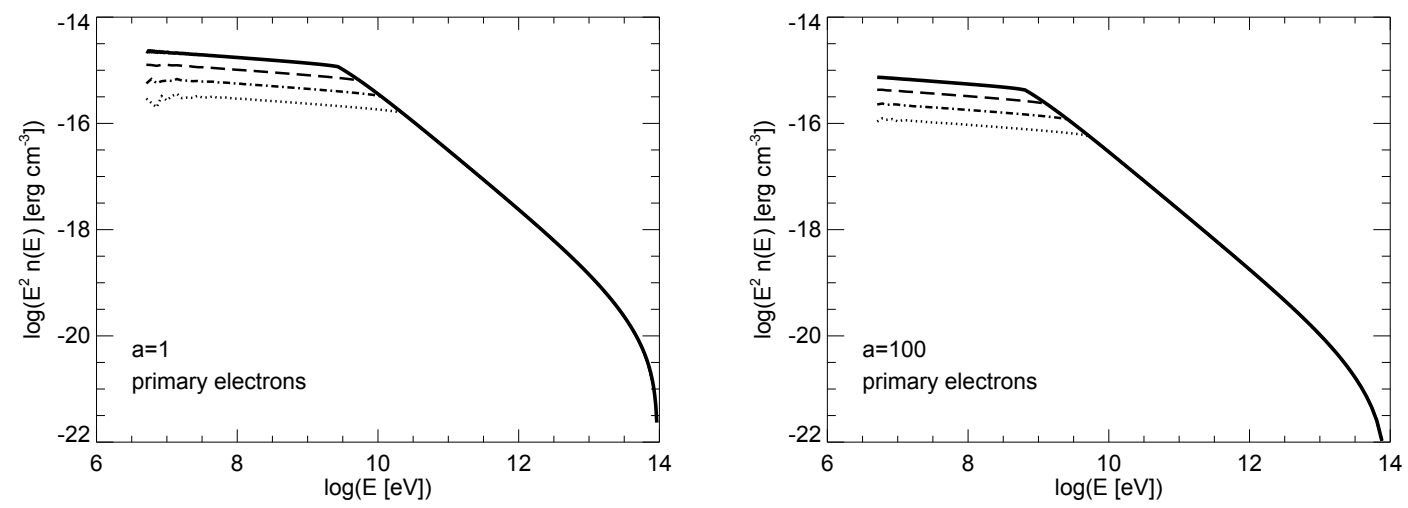

Figura 7.7: Distribuciones de energía $\left(\times E_{e_{1}}^{2}\right)$ de los electrones primarios para los casos $a=1$ (derecha) y 100 (izquierda). Se muestran los estados evolutivos a diferentes tiempos de vida de los remanentes $t_{\mathrm{r}}$ : 0.125 Gyr (línea punteada), 0.25 Gyr (línea de puntos y rayas), 0.5 Gyr (línea rayada) y 1 Gyr (línea llena). El estado presente se alcanza para edades $\sim \tau_{\text {vida. }}$.

\section{Pérdidas hadrónicas}

El tiempo de enfriamiento debido a las colisiones inelásticas $p p$ resulta muy largo, $t_{p p} \sim 10^{20} \mathrm{~s}$, debido a las bajas densidades del medio en los bordes del cúmulo. Por esto, la producción de pares $e^{ \pm}$no será muy eficiente en los remanentes. Considerando las pérdidas por $p p$ la energía máxima que se obtiene es $\sim 2 \times 10^{21}$ y $5 \times 10^{21} \mathrm{eV}$ para los casos con $a=1$ y 100, respectivamente. Sin embargo, para energías $E_{p}>5 \times 10^{18} \mathrm{eV}$ la producción de pares por la interacción protón-fotón del CMB es relevante, y para energías más allá de $\sim 5 \times 10^{19} \mathrm{eV}$ las pérdidas por producción de piones son las dominantes (Berezinsky \& Grigorieva, 1988; Kelner et al. 2008). Sin embargo, no es necesario tener en cuenta estos procesos ya que la energía máxima de los protones queda determinada por restricciones adicionales: el tamaño del acelerador y el tiempo de vida de la fuente.

Los valores de las energías máximas hallados tanto para los electrones como para los protones son válidos siempre y cuando éstos permanezcan dentro de la región de aceleración cuyo tamaño definimos como $l_{\mathrm{a}}$. Por esto, las partículas deben satisfacer el requerimiento $r_{\mathrm{g}}<l_{\mathrm{a}}$. Asumiendo que las partículas se aceleran en los choques trazados por los remanentes observados en frecuencias radio, adoptamos para el tamaño del acelerador $l_{\mathrm{a}} \simeq l_{\mathrm{r}} \sim 0.3 \mathrm{Mpc}$. Debido a que el valor de la energía máxima de los protones obtenido en el párrafo anterior a partir de las pérdidas radiativas corresponde a $r_{\mathrm{g}}>l_{\mathrm{r}}$, determinamos la energía máxima imponiendo que $r_{\mathrm{g}}=l_{\mathrm{r}}$, con lo cual obtenemos $E_{p}^{\max } \sim 10^{20} \mathrm{y}$ $2.6 \times 10^{20} \mathrm{eV}$, para $a=1$ y 100 , respectivamente. Sin embargo, el tiempo requerido para que los protones alcancen esta energía mediante el mecanismo de aceleración de Fermi es mayor que el tiempo de vida de los remanentes, $\tau_{\text {vida }} \sim 1$ Gyr. Así, la verdadera energía máxima hasta la cual pueden acelerarse los protones en los remanentes se obtiene igualando 

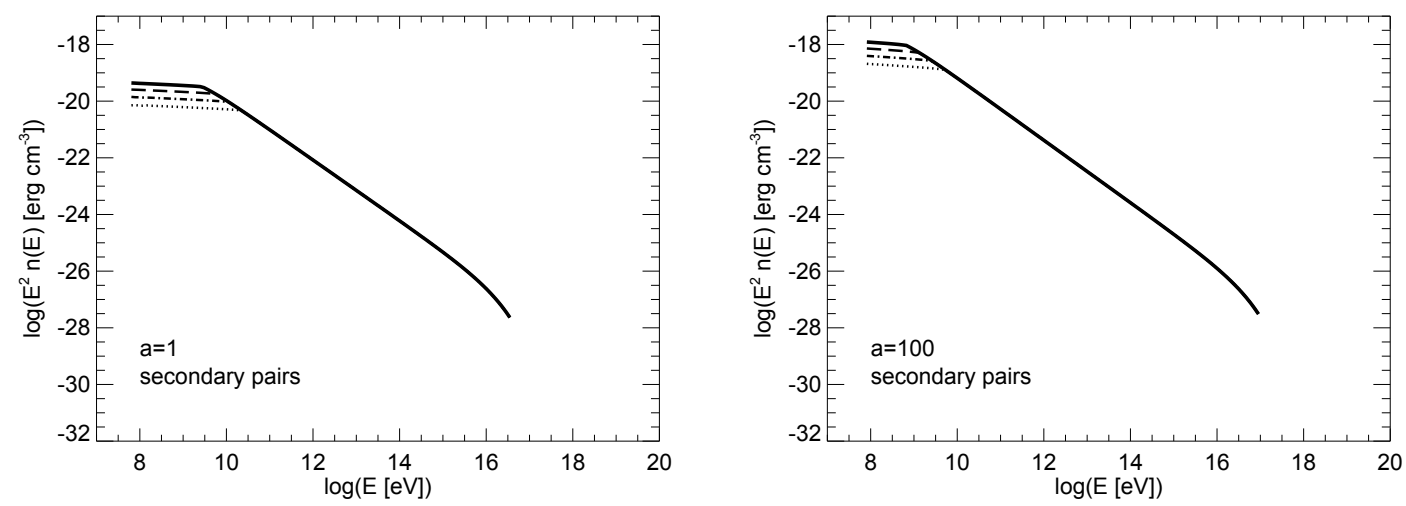

Figura 7.8: Distribuciones de energía $\left(\times E_{e_{2}}^{2}\right)$ de los pares secundarios para los casos $a=1$ (derecha) y 100 (izquierda). Se muestran los estados evolutivos a diferentes tiempos de vida de los remanentes $t_{\mathrm{r}}$ : 0.125 Gyr (línea punteada), 0.25 Gyr (línea de puntos y rayas), 0.5 Gyr (línea rayada) y 1 Gyr (línea llena). El tiempo máximo de evolución es $\sim \tau_{\text {vida }}$.

$t_{\text {acc }}$ y $\tau_{\text {vida }}$, dando $E_{p}^{\max } \sim 5 \times 10^{17}$ y $1.3 \times 10^{18} \mathrm{eV}$ para $a=1$ y 100 , respectivamente, como se muestra en la Tabla 7.3 .

La distribución de energía de los protones acelerados en los remanentes queda determinada por el tiempo de vida de los últimos $\tau_{\text {vida }} \sim 1$ Gyr y debido a que $\tau_{\text {vida }} \sim t_{\text {conv }}$ podemos considerar que $n_{p}\left(E_{p}\right)$ alcanza el estado presente después de un tiempo $\sim \tau_{\text {vida }}$. Por esto,

$$
n_{p}\left(E_{p}\right) \sim q_{p}\left(E_{p}\right) \tau_{\text {vida }} .
$$

Respecto de la producción de pares $e^{ \pm}$, si bien la contribución de éstos a la SED será despreciable, la calcularemos por completitud. Para esto necesitamos conocer como es la distribución energética de estas partículas. La evolución del espectro de inyección de los leptones secundarios en los remanentes queda determinada, al igual que en el caso de los electrones primarios, por las pérdidas radiativas (IC y sincrotrón) para $E_{e_{2}}>E_{\mathrm{q}}$ y por el tiempo de escape para $E_{e_{2}}<E_{\mathrm{q}}$, donde $E_{\mathrm{q}} \sim 5$ y $1 \mathrm{GeV}$, para $a=1$ y 100 , respectivamente. El espectro alcanza el estado de interés, como se muestra en la Figura 7.8, en un tiempo $\sim \tau_{\text {vida }}$.

\subsection{Distribuciones espectrales de energía}

Conociendo las distribuciones de energía $n_{i}$, podemos calcular la emisión que producen las poblaciones de partículas relativistas. Las luminosidades producidas en los remanentes están dadas por la ecuación

$$
E_{\mathrm{ph}} L_{\mathrm{ph}}=E_{\mathrm{ph}}^{2} j_{\mathrm{ph}}\left(E_{\mathrm{ph}}\right) V_{\mathrm{r}},
$$

donde $V_{\mathrm{r}} \sim 0.1 \mathrm{Mpc}^{3}$ es el volumen de la región emisora, es decir, de los remanentes. 


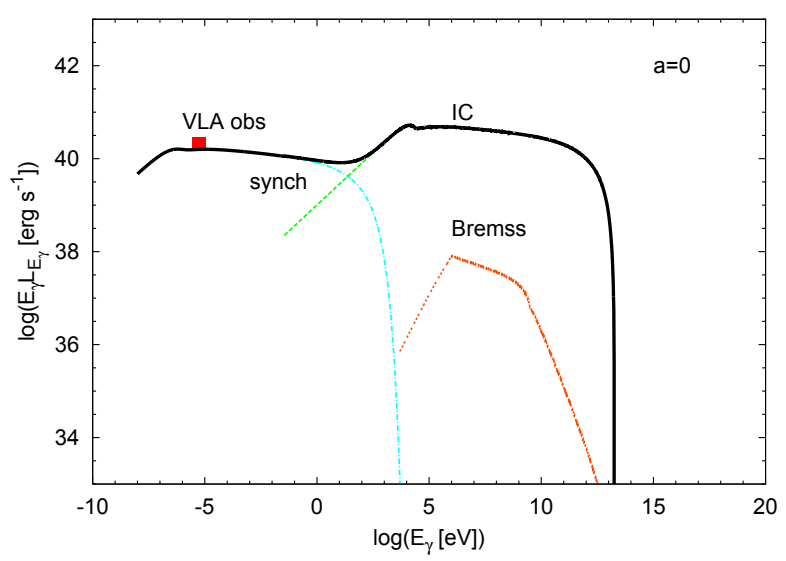

Figura 7.9: Distribución espectral de energía para el caso puramente leptónico, $a=0$. La línea negra representa la suma de las tres contribuciones (sincrotrón, IC y Bremsstrahlung relativista) a la SED total. La luminosidad observada con VLA a la frecuencia $\nu=1.4 \mathrm{GHz}$ del cúmulo Abel 3376 se representa con un cuadrado rojo.

\subsubsection{Interacciones leptónicas}

Las emisividades diferenciales $j_{\mathrm{ph}}\left(E_{\mathrm{ph}}\right)$ producidas por los leptones por radiación sincrotrón, dispersiones IC y Bremsstrahlung relativista son calculadas usando las fórmulas dadas en el Capítulo 3. Las luminosidades específicas son halladas a través de la ecuación (7.8). En los casos $a=1$ y 100, en los cuales se asume que existe una población de protones relativistas, las contribuciones de los pares secundarios a las SEDs también se estiman por completitud.

Los resultados de las luminosidades específicas producidas en el caso donde sólo se aceleran electrones, caracterizado por el parámetro $a=0$, se muestran en la Figura 7.9 , Podemos ver que las interacciones IC son el proceso radiativo más importante, con una luminosidad $L_{\mathrm{IC}} \sim 9.1 \times 10^{41} \mathrm{erg} \mathrm{s}^{-1}$, a energías $E_{\mathrm{ph}} \geq 0.1 \mathrm{MeV}$ y con un cut-off en $\sim 10 \mathrm{TeV}$. La luminosidad producida por Bremsstrahlung relativista es despreciable, en concordancia con los resultados mostrados en la Figura 7.6

\subsubsection{Interacciones hadrónicas}

La emisividad correspondiente a las interacciones $p p$ se calcula considerando las ecuaciones (3.39) y (3.41), y luego la luminosidad específica se calcula a través de la ecuación (7.8). Los resultados de nuestros cálculos se muestran en la Figura 7.10.

En el caso $a=1$, de la misma manera que como ocurre en el caso $a=0$, la SED está dominada por las interacciones IC, con una luminosidad $L_{\mathrm{IC}} \sim 7.4 \times 10^{41} \mathrm{erg} \mathrm{s}^{-1}$. La emisión a energías mayores que $\sim 1 \mathrm{GeV}$ es producida por el decaimiento de piones neutros, alcanzando una luminosidad $L_{p p} \sim 1.6 \times 10^{38} \mathrm{erg} \mathrm{s}^{-1}$ con un cut-off en $E_{\mathrm{ph}} \sim 10^{17} \mathrm{eV}$. Sin embargo, su contribución a la SED es evidente para energías $>10 \mathrm{TeV}$, como se muestra 

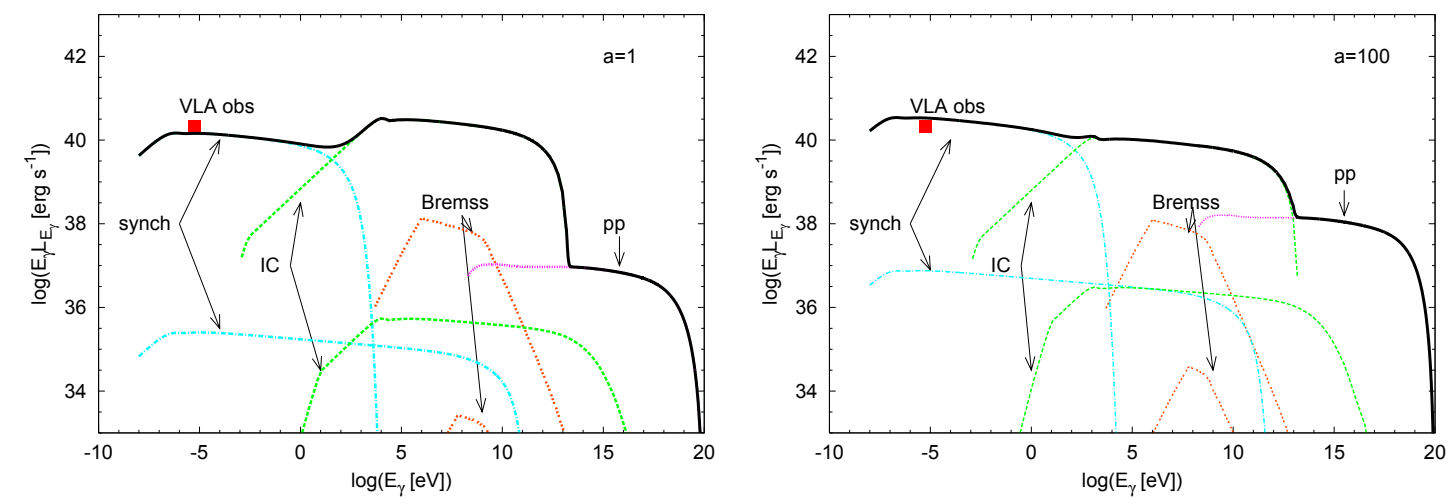

Figura 7.10: Distribuciones espectrales de energía para los casos con $a=1$ (izquierda) y 100 (derecha), en los cuales consideramos tanto una población leptónica como hadrónica de partículas primarias y la población de pares $e^{ \pm}$producida. La emisión producida por los pares secundarios es menor que la correspondiente a los electrones primarios. Como mencionamos en la Figura 7.9, la línea negra indica la SED total (suma de todas las contribuciones, leptónicas y hadrónica) y el cuadrado rojo indica la luminosidad observada con VLA.

en la Figura 7.10 (izquierda).

En el caso correspondiente al valor $a=100$, tenemos que $u_{p}>u_{e_{1}}$, pero esto no implica un flujo significativo de fotones producidos en las interacciones $p p$ ya que la densidad de partículas blanco es constante y muy baja en la localización de los remanentes. Por esto, el espectro de rayos gamma producidos por el decaimiento de los $\pi^{0}$ no domina la SED, como puede observarse en la Figura 7.10 (derecha). La luminosidad $L_{p p}$ es $\sim 4.2 \times 10^{39} \mathrm{erg} \mathrm{s}^{-1}$, siendo apenas mayor que la correspondiente al caso con $a=1$. Por otro lado, contrariamente a lo que ocurre en los casos con $a=0$ y $a=1$, la emisión por IC es menor que la radiación sincrotrón, con luminosidades $L_{\mathrm{IC}} \sim 7.1 \times 10^{40} \mathrm{erg} \mathrm{s}^{-1}$ y $L_{\mathrm{sin}} \sim 3.8 \times 10^{41} \mathrm{erg} \mathrm{s}^{-1}$, respectivamente. El hecho de que la radiación por dispersiones IC sea reducida en el caso con $a=100$ puede explicarse como una consecuencia de la equipartición asumida entre la densidad de energía magnética y de las partículas relativistas. Como puede verse en la Tabla 7.2 el campo magnético se hace más intenso cuando $a$ crece. Esto reduce la cantidad de energía de los electrones que se radía por interacciones IC en comparación con la que se pierde a través del proceso sincrotrón. Además, la densidad de energía en electrones relativistas también se reduce para poder ajustar el espectro con el flujo observado con un valor de $B_{\mathrm{r}}$ mayor. Esto último hace que $L_{\mathrm{IC}}$ sea menor en términos absolutos en el caso $a=100$.

Respecto de la emisión producida por los pares $e^{ \pm}$, encontramos que sus contribuciones a la SED por los diferentes procesos expuestos en la sección 7.3.1 son mayores en el caso con $a=100$ que con $a=1$, pero aún son irrelevantes. Esto es consistente con la baja eficiencia de las interacciones $p p$ que tienen lugar en la periferia del cúmulo. 


\subsection{Discusión}

En este capítulo hemos presentado el estudio realizado sobre el cúmulo de galaxias Abell 3376. Esta fuente presenta fuertes evidencias de una actividad de fusión, caracterizada por grandes estructuras detectadas en frecuencias radio en la periferia del cúmulo y que han sido asociadas con remanentes (Bagchi et al. 2006). Hemos modelado la emisión producida en estos remanentes en un amplio rango de frecuencias, desde radio hasta rayos gamma, considerando la contribución de diferentes procesos radiativos y diferentes relaciones entre las densidades de energía de los protones y los electrones relativistas.

Las pérdidas leptónicas más importantes que afectan la distribución de partículas son la radiación sincrotrón y las dispersiones IC con fotones del CMB. Los protones relativistas se enfrían por interacciones $p p$ con el ICM, produciendo rayos gamma e inyectando una población de pares $e^{ \pm}$los cuales se enfrían por los mismos procesos que los electrones primarios. Los parámetros involucrados en estos procesos no térmicos, como el campo magnético y las distribuciones de partículas relativistas, se han estimado a través del flujo observado en radio de la fuente y de suponer equipartición de la energía magnética y no térmica. Además, las observaciones en rayos X nos permiten extraer información sobre el volumen de los remanentes. Por otro lado, los resultados de simulaciones numéricas nos proveen de valores típicos para la densidad del medio en los bordes del cúmulo, como así también de la velocidad de los choques de fusión.

Las SEDs mostradas en las Figuras 7.9 y 7.10 se han calculado considerando la contribución de diferentes procesos radiativos no térmicos en un amplio rango de energías, desde radio hasta rayos gamma. En este capítulo hemos estudiado particularmente la detectabilidad del cúmulo Abell 3376 en HE y VHE. Encontramos que los procesos radiativos más importantes que contribuyen a la emisión de rayos gamma son las interacciones IC y el decaimiento de $\pi^{0}$, siendo la luminosidad de este último mucho menor que la alcanzada por el primero, aún en el caso en el cual $u_{p}=100 u_{e_{1}}$. La luminosidad por $p p$ obtenida en este caso es $L_{p p} \sim 4 \times 10^{39} \mathrm{erg} \mathrm{s}^{-1}$. La luminosidad por dispersiones IC de fotones del $\mathrm{CMB}$ es $L_{\mathrm{IC}} \sim 7.4 \times 10^{41} \mathrm{erg} \mathrm{s}^{-1}$. Así, los rayos gamma emitidos en el cúmulo Abell 3376, y bajo las suposiciones de nuestro modelo, podrían ser detectables con Fermi y HESS, que operan en las bandas (aproximadas) $100 \mathrm{MeV}-100 \mathrm{GeV}$ y $0.1-10 \mathrm{TeV}$, respectivamente. El futuro telescopio CTA podría permitir incluso la detección de la componente hadrónica a más altas energías.

Finalmente, la proximidad del cúmulo Abell 3376 y su posible contenido alto de partículas relativistas hacen esta fuente interesante para ser observada tanto con instrumentos que actualmente están funcionando como así también con futuros telescopios Cherenckov ya planeados. Notamos que hasta el día de hoy no se han detectado cúmulos de galaxias en rayos gamma y que la fuente Abell 3376 es uno de los mejores candidatos para ser observado en esta banda de energía. 


\section{Capítulo 8}

\section{Conclusiones y perspectivas}

A lo largo de esta tesis hemos estudiado los procesos no térmicos que tienen lugar en diferentes fuentes astrofísicas, tanto galácticas (YSOs, MQs) como extragalácticas (AGNs, cúmulos de galaxias). A continuación resumimos las investigaciones realizadas en cada tipo de fuente y luego damos las conclusiones generales de la tesis.

- YSOs: Algunas estrellas masivas en formación presentan jets y cuando éstos son frenados por el material de la nube molecular en la cual se hallan embebidos, se producen choques terminales fuertes que pueden acelerar partículas hasta energías relativistas. Estas partículas emiten en frecuencias radio por mecanismo sincrotrón y es así como se detectan lóbulos no-térmicos en las regiones terminales de los jets que emanan de protoestrellas de gran masa.

Hemos modelado uno de los lóbulos que forman parte el sistema triple en radio asociado a la fuente IRAS 16547-4247, la protoestrella más luminosa detectada a la fecha $\left(L_{\star} \sim 6.2 \times 10^{4} L_{\odot}\right)$. Suponiendo equipartición entre las densidades de energía magnética y de las partículas relativistas y valiendonós de los flujos observados en frecuencias radio hemos estimado el campo magnético y las distribuciones espectrales de energía para diferentes relaciones entre las densidades de energía de los protones y electrones acelerados en el lóbulo. Luego, calculamos las SEDs considerando diferentes procesos radiativos no térmicos.

Los procesos dominantes resultan ser Bremsstrahlung relativista y las interacciones $p p$, ya que la densidad de la nube molecular es muy grande, $\sim 10^{5} \mathrm{~cm}^{-3}$. Los niveles de emisión predichos son $\sim 10^{32}-10^{33} \mathrm{erg} \mathrm{s}^{-1}$ en altas y muy altas energías, con lo cual la fuente sería detectable con el satélite Fermi y con el futuro arreglo de telescopios Cherenkov CTA.

Las protoestrellas de gran masa, de ser detectadas en altas energías, serían un nuevo tipo de fuentes de rayos gamma. De esta manera, se abre una nueva ventana del espectro electromagnético a través de la cual se puede obtener información sobre como es el proceso de formación de las estrellas tempranas. Este proceso aún hoy no está claramente establecido. Específicamente, se podrán estudiar con nuevas herra- 
mientas las propiedades físicas (como la densidad y campo magnético) de los jets, así como las del medio cincundante.

- MQs: Existen evidencias observacionales de que los vientos de las estrellas de gran masa no son homogéneos sino que tienen una estructura porosa. En los HMMQs, algunos clumps del viento de la estrella compañera pueden llegar hasta los jets generados por el objeto compacto. Debido a la interacción del clump con el material del jet se producen dos choques: uno en el jet y otro en el clump. En el primero se pueden acelerar partículas que luego radiarán localmente por diferentes procesos no térmicos, y el segundo calienta el material del clump, que radía térmicamente. Las partículas aceleradas en el bow shock se enfrían tanto en el jet como en el clump, ya que las más energéticas se difunden hasta allí. Hemos calculado los procesos dinámicos y radiativos más importantes, considerando diferentes valores para el tamaño de los clumps y para el campo magnético en la región del jet donde ocurre la interacción $\left(\sim a_{\mathrm{mq}} / 2\right)$.

De acuerdo a un estudio de la dinámica de la interacción jet-clump, hemos podido estimar la velocidad del choque en el clump y predecimos que la nube no será destruída antes de que las partículas aceleradas en el bow shock se enfríen significativamente. Considerando diferentes valores para el tamaño de los clumps $\left(R_{\mathrm{c}}=10^{10}\right.$ y $\left.10^{11} \mathrm{~cm}\right)$ y del campo magnético en la región de interacción $\left(B_{\mathrm{bs}}=150\right.$ y $\left.1 \mathrm{G}\right)$, hemos calculado las SEDs, prediciendo emisión significativa a lo largo de todo el espectro electromagnético. Las luminosidades más altas obtenidas en rayos gamma son las producidas por interacciones IC con los fotones emitidos por la estrella compañera, $L_{\mathrm{IC}} \sim 10^{35} \mathrm{erg} \mathrm{s}^{-1}$, en HE, y en VHE se han alcanzado luminosidades tan altas como $L_{p p} \sim 10^{32} \mathrm{erg} \mathrm{s}^{-1}$ producidas por colisiones $p p$ en el clump.

De acuerdo al factor de llenado de clumps en el viento que hemos supuesto $(f \sim$ 0.005), el número de clumps que simultáneamente pueden interactuar con el jet es $\sim 350$ si $R_{\mathrm{c}}=10^{10} \mathrm{~cm} \mathrm{y} \sim 0.5$ si $R_{\mathrm{c}}=10^{11} \mathrm{~cm}$. Luego, la emisión será estacionaria en el primer caso, con una luminosidad total $\sim 350$ veces más alta que la de una interacción simple, con una escala de tiempo de los flares de $\sim 1$ hora, y recurrencia en el plazo de varias horas. De esta manera, las interacciones jet-clump son una posible explicación a la producción de los flares observados en algunas binarias de rayos gamma.

Los niveles de emisión alcanzados son detectables por intrumentos como Fermi en HE y los telescopios Cherenkov de nueva generación en VHE. Esta emisión, de ser detectada, nos proveería importante información sobre los jets de los HMMQs, como así también de los vientos de las estrellas de gran masa (e.g. Cygnus X-1, Cygnus X$3)$.

- AGNs: Un estudio similar al realizado sobre los HMMQs ha sido desarrollado en el escenario de los AGNs, considerando que nubes de la BLR pueden penetrar en los jets que emanan de las cercanías del SMBH. Debido a la interacción de una nube 
con uno de los jets se produce un bow shock fuerte en el jet, donde pueden acelerarse partículas eficientemente, y otro choque más débil en la nube. Como en el caso de los HMMQs, las partículas aceleradas en el bow shock se enfrían tanto en el jet como en la nube, ya que las más energéticas se difunden hasta allí.

Los procesos dinámicos que se desarrollan permiten que la nube entre y permanezca entera suficiente tiempo como para que las partículas relativistas aceleradas en el bow shock puedan radiar una fracción significativa de su energía. Las luminosidades obtenidas por la interacción de una sola nube con el jet son relativamente bajas, $\sim 10^{38} \mathrm{erg} \mathrm{s}^{-1}$, pero si muchas nubes se encuentran simultáneamente interactuando con ambos jets entonces la contribución total es significativa y detectable en fuentes no alineadas (e.g. radiogalaxias). Debido a que el emisor está practicamente quieto, la radiación no es amplificada por efecto Doppler como acurre en la emisión producida en los jets de los blazares. La interacción de nubes de la BLR con jets podría ser un mecanismo que explique la emisión observada en algunos AGNs.

Finalmente, hemos aplicado nuestro modelo a galaxias FR I y FR II. En las primeras, si bien no hay detecciones claras de la presencia de una BLR, es posible suponer una población de nubes oscuras aunque no podemos estimar el número de ellas. Por esto, consideramos aquí sólo la interacción de una nube con el jet. Debido a que estas fuentes son cercanas (Cen A, M 87) la interacción de una sola nube de tamaño $R_{\mathrm{n}} \sim 10^{14} \mathrm{~cm}$ podría producir flares detectables. Sin embargo, en las galaxias FR II la cantidad de nubes es grande, y la interacción de muchas de ellas con ambos jets del AGN puede producir emisión detectable si los jets no están alineados con la línea de la visual.

- Cúmulos de galaxias: Los cúmulos de galaxias son candidatos a ser fuentes de rayos gamma, ya que hay evidencias de la existencia de partículas relativistas que emiten en frecuencias radio. En particular, en esta tesis hemos estudiado la producción de rayos gamma en los remanentes (relics) detectados en el borde de la fuente cercana Abell 3376. Los remanentes son trazadores de choques de fusión. El cúmulo Abell 3376 presenta grandes estructuras en frecuencias radio, y que han sido asociadas a remanentes de choques producidos por la fusión entre dos cúmulos de galaxias. Estos remanentes sugieren un rico contenido de partículas relativistas del ICM en estas estructuras. Suponemos que la aceleración de partículas tiene lugar en los bordes de los cúmulos donde se detectan los remanentes radio. Estas partículas luego se enfrían por diferentes procesos no térmicos.

Además de los electrones primarios y protones acelerados en los choques, consideramos también una población de pares secundarios generados por interacciones $p p$ de los protones relativistas con el ICM. Los parámetros involucrados en los procesos no térmicos han sido estimados considerando los datos observacionales como así también suposiciones como la de equipartición de la energía magnética y no térmica. Por otro lado, hemos hecho uso de simulaciones numéricas para obtener aquellos parámetros del medio (velocidad del choque y densidad del ICM) no provistos por 
las observaciones.

La emisión no térmica más intensa producida en los bordes de este cúmulo es debida a las interacciones IC de electrones primarios con los fotones del CMB, alcanzando luminosidades $\sim 10^{41} \mathrm{erg} \mathrm{s}^{-1}$ en HE y VHE. Si bien al día de hoy no se han detectado cúmulos de galaxias en rayos gamma, la fuente Abell 3376 es un buen candidato para ser detectado por medio de exposiciones prolongadas de acuerdo a los resultados de nuestro modelo y a su proximidad.

Las ondas de choque se producen en diferentes tipos de fuentes astrofísicas y por diversos mecanismos. Los frentes de choque asociados a estas ondas tienen tamaños que van desde $\sim 10^{-2} \mathrm{pc}$ en los jets de los YSOs hasta $\sim 1 \mathrm{Mpc}$ en los cúmulos de galaxias. Sin embargo, las velocidades de estos choques no son proporcionales a su tamaño, ya que en YSOs y en cúmulos de galaxias $v_{\mathrm{ch}} \sim 1000 \mathrm{~km} \mathrm{~s}^{-1}$ mientras que en los jets relativistas de los MQs y AGNs $v_{\text {ch }} \sim c$. En todos estos choques se pueden acelerar partículas cargadas hasta velocidades relativistas. El tamaño de los frentes de choque influye en las pérdidas de escape, que se tornan muy lentas si aquéllos son muy grandes. El tamaño también es relevante para las densidades de energía de los campos ambientales. Si las densidades de energía magnética y de partículas (fotones y materia) son bajas, entonces las pérdidas radiativas tampoco son muy eficientes y las energías máximas serán altas, $>1 \mathrm{PeV}$. Sin embargo, si las densidades de energía de los campos ambientales son altas, es posible alcanzar energías máximas de $\sim 1-10 \mathrm{TeV}$ en el régimen de saturación.

La competencia entre los tiempos dinámicos y radiativos determina la eficiencia radiativa de la fuente. Si $t_{\mathrm{rad}}<t_{\text {din }}$ en algún rango de energías de la distribución de partículas relativistas, entonces las partículas con esas energías radiarán significativamente y se dice que han alcanzado el régimen de saturación. En todos los escenarios explorados en esta tesis, el régimen de saturación es alcanzado por los electrones en algún rango de energía y para algún conjunto de parámetros considerado. Sin embargo, sólo en aquellos casos en los cuales las partículas más energéticas son las que saturan, la emisión de la fuente resulta intensa y detectable. De ser confirmados los resultados hallados en cada tipo de fuente que hemos estudiado, se aportarían nuevos conocimientos en cada campo de investigación respectivo.

En lo que refiere a las estrellas de gran masa, por un lado modelamos la emisión de la protoestrella asociada a la fuente IRAS 16547-4247 obteniendo flujos detectables en rayos gamma, lo cual sólo había sido someramente sugerido anteriormente por Henriksen y colaboradores (1991), y siendo así estos objetos un nuevo tipo de posibles de fuentes de rayos gamma. Esto ha sido estadísticamente comprobado a través de la muy buena correlación encontrada entre las fuentes detectadas por Fermi y la localización en el plano del cielo de protoestrellas de gran masa (Munar y colaboradores, comunicación personal).

Por otro lado, las propiedades de las inhomegeneidades de los vientos de las estrellas tempranas pueden conocerse a través de observaciones en rayos gamma, ya que las interacciones de éstas con los jets de los MQs producirían efectos detectables. Para poder determinar la microestructura de la variabilidad es necesario el uso de telescopios con gran 
resolución temporal (gran sensibilidad). Un instrumento apropiado para esto será el arreglo de telescopios Cherenkov CTA.

Las interacciones de obstáculos con jets nos proveen un nuevo mecanismo de radiación, ya que además de acelerarse las partículas en los bow shocks que se forman en los jets, el obstáculo sirve de blanco para las interacciones de estas partículas relativistas. En el campo de las binarias de rayos gamma de alta masa, el proceso descripto puede ocurrir si los clumps del viento de la estrella compañera llegan hasta el jet generado por el agujero negro y así podrián producirse los flares detectados en algunas de estas fuentes. En el campo de las galaxias activas, las interacciones de nubes de la BLR con la base de los jets podrían explicar la emisión esporádica observada en galaxias FR II cercanas y la emisión estacionaria de los AGNs más lejanos que no son blazares. Como trabajo a futuro en este campo, haremos una aplicación del modelo expuesto en el Capítulo 6 a la fuente 3C 120, de la cual contamos con datos observacionales de alta resolución espacial. De estos datos es posible extraer información sobre la microestructura del jet, que presenta inhomogeneidades de $\sim \mathrm{cm}$ (Gómez et al. 2008). Como una consecuencia natural de los modelos desarrollados de interacciones de jets con obstáculos, nos proponemos estudiar que sucede cuando una estrella masiva interactúa con el jet de un AGN. Si bien aquí el choque en el jet se produciría por la colisión entre el viento de la estrella y el material del jet, gran parte del modelo ya desarollado puede aplicarse a este nuevo escenario.

En el tópico de los cúmulos de galaxias ofrecemos un candidato concreto para ser observado con los telescopios de rayos gamma. A través de una modelización de la fuente Abell 3376, estimamos que el flujo emitido por ésta sería detectable por los instrumentos actuales que observan en HE como así también por los futuros telescopios ya planeados. Sin embargo, el satélite Fermi no ha detectado ningún cúmulo de galaxias en tres años de observación. Esto muestra que alguno de los valores de los parámetros supuestos por los modelos actuales está siendo sobreestimado. Debido a que el efecto más importante es el IC sobre los fotones del CMB, y el valor de $u_{\text {cmb }}$ está bien determinado, es lícito pensar que quizás la población de partículas relativistas no es tan importante. Esto podría ocurrir si los choques de fusión no fuesen tan intensos como resulta de las simulaciones numéricas y la velocidad de éstos fuese menor que $1000 \mathrm{~km} \mathrm{~s}^{-1}$. Como trabajo a futuro en este campo, nos proponemos hacer un análisis del espacio de valores de los parámetros considerados en la modelización de la emisión de rayos gamma de los cúmulos de galaxias.

Finalmente, de ser detectadas las fuentes propuestas en esta tesis y de comprobarse los resultados obtenidos al estudiar las interacciones de jets con obstáculos, se incrementarían las clases de emisores de rayos gamma y se podrían explicar la emisiones esporádicas producidas en algunas fuentes, mediante mecanismos no explorados anteriormente. 



\section{Referencias}

Abdo, A.A. et al. (Fermi Collaboration) 2009a, ApJS, 183, 46

Abdo A. A. et al. (Fermi Collaboration) 2009b, ApJ, 707, 1310

Aharonian, F.A., Atoyan, A.M., 2000, A\&A, 362, 937

Aharonian, F.A., et al., 2005, Science, 309, 746

Aharonian, F.A., et al., 2007, A\&A, 467, 1075

Aharonian, F. A., Anchordoqui, L. A., Khangulyan, D., \& Montaruli, T. 2006, J.Phys.Conf.Ser., 39, 408

Aharonian, F.A. et al. (H.E.S.S. Collaboration) 2007, A\&A, 467, 1075

Aharonian, F.A. et al. (H.E.S.S. Collaboration) 2009, ApJ, 695L, 40

Albert, J., et al. 2006, Science, 312, 1771

Albert, J., et al., 2007, ApJ, 665, L51

Alexander, D.M., Hough, J.H., Young, S., Bailey, J.A., Heisler, C.A., Lumsden S.L.,

Robinson, A., 1999, MNRAS 303, L17

Araudo A., Romero G.E., Bosch-Ramon V., Paredes J.M. 2007, A\&A, 476, 1289

Araudo A., Romero G.E., Bosch-Ramon V., Paredes J.M., 2008a, IJMPD, 17, 1889

Araudo A., Cora S., Romero G.E. 2008b, MNRAS, 390, 323

Araudo, A. T., Bosch-Ramon, V., Romero, G. E. 2009, A\&A, 503, 673

Araudo, A. T., Bosch-Ramon, V., Romero, G. E. 2010, A\&A (en prensa) [arXiv:1007.2199]

Atoyan A.M., Völk H.J., 2000, ApJ, 535, 45

Axford, W. I.; Leer, E.; Skadron, G., 1977, ICRC 11, 132

Bagchi J., Durret F., Neto G.B.L., Paul S., 2006, Sci, 314, 791

Balestra I., Tozzi P., Ettori S., Rosati P., Borgani S., Mainieri V., Norman C., Viola M., 2007, A\&A, 462, 429

Bell, A.R., 1978, MNRAS, 182, 147

Bentz, M.C., Peterson, B.M., Pogge, R.W., Vestergaard, M., Onken, C.A., 2006, ApJ, 644, 133

Berezinsky V.S. \& Grigorieva S.I., 1988, A\&A, 199, 1

Berrington, R.C. \& Dermer C.D., 2003, ApJ, 594, 709

Blake, G.M., 1972, MNRAS 156, 67

Blandford, R.D. \& Znajek, R.L., 1977, MNRAS 179, 433

Blandford, R.D. \& Payne, D.G., 1982, MNRAS 199, 883

Blondin, J.M., Königl, A., Fryxell, B.A., ApJ, 1989, 337L

Blumenthal, G.R., Gould, R.J., 1970, Rev. Mod. Phys., 42, 237 
Bonnell, I.A., Bate, M.R., Zinnecker, H., 1998, MNRAS, 298, 93

Bosch-Ramon, V., Aharonian, F.A., \& Paredes, J.P., 2005, A\&A, 432, 609

Bosch-Ramon, V., Romero, G.E., Paredes, J.M., 2005, A\&A, 429, 267

Bosch-Ramon, V., Romero, G.E., \& Paredes, J.P., 2006, A\&A, 447, 263

Bosch-Ramon, V., 2006, Tesis doctoral: Broadband emission from high energy processes in microquasars

Bosch-Ramon, V., Motch, C., Ribó, M., Lopes de Oliveira, R., Janot-Pacheco, E.,

Negueruela, I., Paredes, J.M., \& Martocchia, A., 2007, A\&A, 473, 545

Bosch-Ramon, V. \& Khangulyan, D., 2009, IJMPD, 18, 347

Bosch-Ramon, V., Romero, G.E., Araudo, A.T., Paredes, J.M., 2010, A\&A, 511, 8

Bowyer S., Korpela E.J., Lampton M., Jones T.W., 2004, ApJ, 605, 168

Brooks, K., Garay G., Mardones, D., Bronfman, L., 2003, ApJ, 594, L131

Cao, X., Jiang, D.R., 1999, MNRAS, 307, 802

Churazov E., Forman W., Jones C., Böhringer H., 2000, A\&A, 356, 788

Coppi, P., Blandford R., 1990, MNRAS, 245, 453

Corbel, S., Fender, R.P., Tzioumis, A.K., Tomsick, J.A., Tomsick, J.A., Orosz, J.A., Miller

J.M., Wijnands, R., Kaaret, P., 2002, Sci, 298, 196

Courvoisier, T.J.-L. 1998, A\&A Rv, 9, 1

de Jager, O.C., Harding, A.K., Michelson, P.F., Nel, H.I., Nolan, P.L., Sreekumar, P., Thompson, D.J., 1996, ApJ, 457, 253

Dietrich, M., Wagner, S.J., Courvoisier, T.J.-L., Bock, H., North, P., 1999, A\&A, 351, 31

Dolag K., Vazza F., Brunetti G., Tormen G., 2005, MNRAS, 354, 753

Domainko W., Benbow W., Hinton J.A., Martineau-Huynh O., de Naurois M., Nedbal

D., Pedaletti G., Rowell G., for the H. E. S. S. Collaboration 2007, 30th International

Cosmic Ray Conference, Merida, Mexico, astro-ph/0708.1384v1

Drury, L.O.'C., 1983, RPPh, 46, 973

En $\beta$ lin T. A., Biermann P. L., 1998, A\&A, 330, 90

En $\beta$ lin T. A., Biermann P. L., Klein U., Kohle S. 1998, A\&A, 332, 395

En $\beta$ lin T. A., Gopal-Krishna, 2001, A\&A, 366, 26

Evans, D.A., Kraft, R.P., Worrall, D.M., Hardcastle, M.J., Jones, C., Forman, W.R.,

Murray, S.S., 2004, ApJ, 612, 786

Fanaroff, B.L.,Riley, J.M.: 1974, MNRAS 167, 31

Fegan S.J., Badran H.M., Bond I.H., Boyle P.J., Bradbury S.M., Buckley J.H.,

Carter-Lewis D.A., Catanese M., et al., 2005, ApJ, 624, 638

Feretti L., Givannini G., 1996. In R. Ekers, C. Fanti L̂. Padrielli (eds.) IAU Symp. 175,

Extragalactic Radio Sources. Kluwer Academic Publisher, p. 333

Feretti L., Böhringer H., Giovannini G., Neumann D., 1997, A\&A, 317, 432

Feretti L., Fusco-Femiano R., Giovannini G., Govoni F., 2001, A\&A, 373, 106

Feretti L., Burigana C., En $\beta$ lin T.A., 2004, New Astron. Rev., 48, 1137

Feretti L., Giovannini G., 2008, in Plionis M., Lopez-Cruz O., Hughes D. (eds.)

Panchromatic View of Clusters of Galaxies and the Large-Scale Structure, Lecture

Notes Physics 740, Springer, Dordrecht, p. 143

Fermi, E., 1949, Physics Review 75, 1169 
Ferrari, C., 2010 (en prensa) [arXiv:1005.3699]

Fragile, P.C., Murray, S.D., Anninos, P. \& van Breugel, W., 2004, ApJ, 604, 74

Fusco-Femiano R., dal Fiume D., Feretti L., Giovannini G., Grandi P., Matt G., Molendi S., Santangelo A., 1999, ApJ, 513, L21

Fusco-Femiano R., Orlandini M., Brunetti G., Feretti L., Giovannini G., Grandi P., Setti G., 2004, ApJ, 602, L73

Gabici S., Blasi P., 2003, ApJ, 583, 695

Gallo, E., Fender, R., Kaiser, C., Russell, D., Morganti, R., Oosterloo, T., \& Heinz, S., 2005, Natur, 436, 819

Garay, G.; Rodriguez, L.F.; Moran, J.M.; Churchwell, E., 1993, ApJ 418, 368

Garay, G., Brooks, K., Mardones, D., Norris, R.P., 2003, ApJ, 537, 739

Garay, G.; Mardones, D.; Bronfman, L.; Brooks, K.J.; Rodríguez, L.F.; Güsten, R.;

Nyman, L.-A.; Franco-Hernández, R.; Moran, J.M.; A\&A, 463, 217

Gaisser, T.K., 1990, Cosmic Rays and Particle Physics, Cambridge University Press, Cambridge

Ginzburg, V.L., Syrovatskii, S.I., 1964, The Origin of Cosmic Rays, Pergamon Press, New York

Giovannini G. Feretti L., Stanghellini C., 1991, A\&A, 252, 528

Giovannini G., Tordi M., Feretti L., 1999, New Astron., 4, 141

Giovanninii G., Ferreti L., 2004, JKAS, 37, 323

Girardi M., Giuricin G., Mardirossian F., Mezzetti M., Boschin W. 1998, ApJ, 505, 74

Ghisellini, G., Maraschi, L., Treves, A. 1985, A\&A, 146, 204

Ghisellini, G., Tavecchio, F., Foschini, L., Ghirlanda, G., Maraschi, L., Celotti, A. 2010, MNRAS, 402, 497

Gómez J.L., Marscher A.P., Jorstad S.G., Agudo I., Roca-Sogorb M., 2008, ApJL, 681, L69

Govoni F, Feretti L., Giovannini G., Böhringer H., Reiprich T.H., Murgia M. 2001, A\&A, 376,803

Govoni F, Feretti L., 2004, Int. J. Mod. Phys. D, 13, 1549

Grandi, P., Palumbo G.G.C., 2004, Sci, 306, 998

Hartman, R.C. et al., 1999, ApJS, 123, 79

Henriksen, R.N.; Mirabel, I.F.; Ptuskin, V.S.; 1991, A\&A, 248, 221

Hillas, A.M., 1984, ARA\&A, 22, 425

Hoeft M., Brüeggen M., Yepes G. 2004, MNRAS, 347, 389

Israel, F.P., 1998, A\&AR, 8, 237

Jaffe W.J., 1977, ApJ, 212, 1

Jokipii, J.R.; 1987, ApJ, 313, 842

Jolley, E.J., Kuncic, Z., Bicknell, G.V., Wagner, S., 2009, MNRAS, 400, 1521

Junor, W., Biretta, J.A., Livio, M. 1999, Nature, 401, 891

Kaspi, S., Maoz, D., Netzer, H., Peterson, B.M., Vestergaard, M., Jannuzi, B.T., 2005, ApJ 629,61

Kaspi, S., Brandt, W.N., Maoz, D., Netzer, H., Schneider, D.P., Shemmer, O., 2007, ApJ 659,997 
Kataoka, J., Tanihata, C., Kawai, N., Takahara, F., Takahashi, T., Edwards, P.G., Makino, F., 2002, MNRAS 336, 932

Khangulyan D., Hnatic S., Aharonian F., Bogovalov S., 2007, MNRAS, 380, 312

Kaufman Bernadó, M.M., Romero, G.E., Mirabel, I.F., 2002, 385, L10

Kelner, S.R., \& Aharonian, F.A., 2008, PhysRevD 78, 034013

Kelner, S.R., Aharonian, F.A., \& Vugayov, V.V., 2006, Phys.Rev.D 74, 034018

Keshet U., Waxman E., Loeb A., Springel V., Hernquist L., 2003, ApJ, 585, 128

Khangulyan, D., Hnatic, S., Aharonian F., Bogovalov S. 2007, MNRAS 380, 320

Khangulyan, D., Aharonian, F., Bosch-Ramon, V., 2008, MNRAS, 383, 467

Klein, R.I., McKee, C.F. \& Colella, P., 1994, ApJ, 420, 213

Krolik, J.H., McKee, C.F., \& Tarter, C.B., 1981, ApJ, 249, 422

Krtička, J., \& Kubát, J., 2001, A\&A, 377, 175

Landau, L.D. \& Lifshitz, E., 1951, The classical theory of fields, Addison Wesleey Press,

Cambridge

Landau, L.D. \& Lifshitz, E., 1959, Fluid Mechanics,

Lang, K.R., 1999, Astrophysical Formulae, Springer, Berlin

Lieu R., Mittaz J.P.D., Bowyer S., Lockman F., Hwang C.-Y., Schmitt J.H.M.M., 1996, ApJ, 458, L5

Lieu R., Axford W. I., Bonamente M., 1999, ApJ, 510, L25

Mannheim, K., Schlickeiser, R., 1994, A\&A, 286, 983

Marconi A., Schreider, E.J., Koekemoer, A., Capetti, A., Axon, D., Macchetto, D., \& Kaon, N., 2000, ApJ, 528, 276

Markevitch, M. et al.; 2000, ApJ, 541, 542

Martí, J., Rodríguez, L.F., Reipurth, B., 1995, ApJ, 449, 184

Miller-Jones, J.C.A., Fender, R.P. \& Nakar, E., 2006, MNRAS, 367, 1432

Mirabel, I.F. \& Rodríguez, L.F., 1999, ARA\&A 37, 409

Mirabel, I.F., Laurent, O., Sanders, D.B., Sauvage, M., Tagger, M., Charmandaris, V., Vigroux, L., Gallais, P., Cesarsky, C., Block, D.L. 1999, A\&A 341, 667

Mittaz J.P.D., Lieu R., Lockman F.J., 1998, ApJ, 498, L17

Moffat, A.F.J., 2008, Proceedings of the conference "Clumping in hot-star winds" Eds.: Hamann, W.R., Feldmeier, A. \& Oskinova, L., 17

Myasnikov, A.V.; Zhekov, S.A.; Belov, N.A., 1998, MNRAS, 298, 1021

Orellana, M., Bordas, P., Bosch-Ramon, V., Romero, G. E., \& Paredes, J. M. 2007, A\&A, 476,9

Orosz, J.A., Miller J.M., Wijnands, R., Kaaret, P., 2002, Sci, 298, 196

Owocki, S.P., \& Cohen D.H., 2006, ApJ, 648, 5650

Owocki, S.P., Romero G.E., Townsend, R. \& Araudo, A.T., 2009, ApJ 696, 690

Pacholczyk, A.G., 1970, Radio Astrophysics, Freeman, San Francisco

Paltani, S., \& Türler, M., 2005, A\&A, 435, 811

Paredes, J.M., Ribó, M., Bosch-Ramón, V., et al., 2007, ApJ, 664, L39

Paredes, J.M. 2008, Int. Jour. Mod. Phys. D, 17, 1849

Penston, M.V.,1988, MNRAS, 233, 601

Perkins J.S., Badran H.M., Blaylock G., Bradbury S.M., Cogan P., Chow Y.C.K., Cui W., 
Daniel M.K., et al., 2006, ApJ, 644, 148

Perucho, M., \& Bosch-Ramon, V, 2008, A\&A, 482, 917

Peterson, B.M.; Bentz, M.C.; Desroches, L.-B.; Filippenko, A.V.; Ho, L.C.; Kaspi, S.; Laor, A.; Maoz, D.; Moran, E.C.; Pogge, R.W.; Quillen, A.C., 2005, ApJ, 632, 799

Peterson, B.M., 2006, LNP 693, 77

Petrosian V., 2001, ApJ, 557, 560

Pfrommer C., Springel V., En $\beta$ lin T. A., Jubelgas M., 2006, MNRAS, 367, 113

Pfrommer C., Enßlin T.A., Springel V., Jubelgas M., Dolag K., 2007, MNRAS, 378, 385

Pfrommer C., En $\beta$ lin T. A., Springel V., 2008, MNRAS, 385, 1211

Pian, E. et al., 1999, ApJ, 521, 112

Platzek, A.M., Apuntes de la materia Introducción a la magnetohidrodinámica

Priest, E.R., 1982, Solar Magnetohydrodynamics, Reidel, Dordrecht

Protheroe, R.J., 1999, Topics in cosmic-ray astrophysics, eds. M. A. DuVernois (Nova

Science Publishing), p. 240 [astro-ph/9812055]

Puls, J., Markova, N., Scuderi, S., Stanghellini, C., Taranova, O.G., Burnley, A.W., Howarth I.D., 2006, A\&A, 454, 625

Raga, A.C., Cantó, J., Rodríguez-González, A. \& Esquivel, A., 2009, A\&A, 493, 115

Reimer O., Pohl M., Sreekumar P., Mattox J. R., 2003, ApJ, 588, 155

Rees, M., 1984, ARA\&A 22, 471

Rees, M.J., 1987, MNRAS, 228, 47

Reynoso, M. \& Romero, G. E. 2009, A\&A, 493, 1

Ribó, M., 2005, ASPC, 340, 269

Rieger, F.M., Aharonian, F.A., 2009, A\&A

Risaliti, G., 2009 [arXiv:0912.2118]

Risaliti, G., Elvis, M., Nicastro, F., 2002, ApJ 571, 234

Rodríguez, L.F., Marti, J., Canto, J., Moran, J.M., Curiel, S., 1993, RMxAA, 25, 23

Rodríguez L.F., Garay G., Brooks, K., Mardones, D., 2005, ApJ 626, 953

Romero, G.E., 1995, Ap\&SS 234, 49

Romero, G.E., Combi, J.A., Perez Bergliaffa S.E., Anchordoqui, L.A., 1996, APh, 5, 279

Romero, G.E., Benaglia, P., Torres, D.F., 1999, A\&A, 348, 868

Romero, G. E. 2001, The Nature of Unindentified Galactic High-Energy Gamma-Ray Sources, ed. A. Carraminana, O. Reimer, \& D. Thompson, Kluwer Academic Publishers, Dordrecht, 65

Romero, G. E., Torres, D. F., Kaufman Bernadó, M. M., \& Mirabel, I. F., 2003, A\&A, 410,1

Romero, G. E. \& Orellana, M. 2005, A\&A, 439, 237

Romero, G.E.; Okazaki, A.T.; Orellana, M.; Owocki, S.P., 2007, A\&A, 474, 15

Romero, G. E., Owocki, S. P., Araudo, A. T., Townsend, R. H. D., \& Benaglia, P., Actas del congreso Clumping in Hot Star Winds, W. R. Hamann, A. Feldmeier \& L. M.

Oskinova (eds.), Potsdam, Univ. Verl., 2008, p. 191

Romero, G.E., del Valle, M.V., Orellana, M., 2010, A\&A, 518, 12

Romero, G.E., 2010, Apuntes de la materia Introducción a la Astrofísica Relativista. (http://www.iar.unlp.edu.ar/garra/AR/apunte.html) 
Röttgering H.J.A., Snellen I., Miley G., de Jong J.P., Hanisch R.J., Perley R., 1994, ApJ, 436, 654

Röttgering H.J.A., Wieringa M.H., Hunstead R.W., Ekers R.D., 1997, MNRAS, 290, 577

Russell, D.M., Fender, R.P., Gallo, E., \& Kaiser, C.R., 2007, MNRAS, 376, 1341

Sabatini, S. et al., 2010, ApJ, 712, L10

Shakura, N.I., Sunyaev, R.A., 1973, A\&A 24, 337

Shin, M.-S., Stone, J. M., Snyder, G. F. 2008, ApJ, 680, 336

Shu, F.H, Adams, F.C., Lizano, S. 1987, ARA\&A, 25, 23

Soldi, S., Beckmann, V., Türler M., 2009 [arXiv:0912.2266v1]

Sturrock, P.A., 1971, ApJ 164, 529

Taub, A.H., 1948, PhRv 74, 328

Tavani, M. et al. 2009a (AGILE Collaboration), ApJ, 698, L142

Tavani, M. et al. 2009b (AGILE Collaboration), Nature, 462, 620

Thomson, R.C., 1992, MNRAS, 257, 689

Tingay S.j. et al., 1998, AJ, 115, 960

Urry, C. \& Padovani, P., 1995, PASP, 107, 803

van Dike \& Gordon, H., 1959, NASA TR R-1

Vila, G., Aharonian F., 2009, en 'Compact Objects and their Emission, Eds: Romero, G.E., Benaglia, P.

Völk H.J., Aharonian F.A., Breitschwerdt D., 1996, SSRv, 75, 279

Wang, B., Inoue, H., Koyama K., Tanaka, Y., 1986, PASJ, 38, 685

Zhekov, S.A. \& Palla, F., 2007, MNRAS, 382, 1124

Zinnecker, H.; Yorke, H.W., 2007, ARA\&A 45, 481 


\section{Apéndice A}

\section{Lista de acrónimos}

Debido al uso generalizado de las siglas inglesas en la jerga astronómica referida al tema de esta tesis, se han utilizado a lo largo de este trabajo una gran cantidad de acrónimos que se refieren a palabras en la lengua mencionada.

AGN: Active Galactic Nucleus

ATCA: Australia Telescope Compact Array

BH: Black Hole

BLR: Broad Line Region

CDM: Cold Dark Matter

CMB: Cosmic Microwave Background

CTA: Cherenkov Telescope Array

EC: External Compton

FR: Faranoff-Rayleigh

FSRQ: flat spectrum radio-quasar

GRB: Gamma-Ray Burst

HE: High Energy

HH: Herbig-Haro

HMMQ: High Mass Microquasar

IC: Inverse Compton

ICM:Intra Cluster Medium IR: Infra Red

KH: Kelvin-Helmholtz

MHD: Magnetohydrodynamics

MQ: Microquasar

RT: Rayleigh-Taylor

SED: Spectral Energy Distribution

SMBH: Super-Massive Black Hole

SPH: Smoothed Particle Hydrodynamics

SSC: Synchrotron Self Compton

VHE: Very High Energy

VLA: Very Large Array 
WR: Wolf-Rayet

YSO: Young Stellar Object 


\section{Apéndice B}

\section{Lista de publicaciones}

- Publicaciones en revistas internacionales con referato

1. Gamma-ray emission from massive young stellar objects

Anabella T. Araudo, Gustavo E. Romero, Valentí Bosch-Ramon \& Josep M. Paredes

A\&A, 476, 1289-1295, 2007

2. Gamma-ray emission from massive star forming regions

Anabella T. Araudo, Gustavo E. Romero, Valentí Bosch-Ramon \& Josep M.

Paredes

IJMPD, 17, 1889-1894, 2008

3. Non-thermal processes in the cluster of galaxies Abell 3376

Anabella T. Araudo, Sofía A. Cora \& Gustavo E. Romero

MNRAS, 390, 323, 2008

4. High-energy emission from jet-clump interactions in microquasars

Anabella T. Araudo, Valentí Bosch-Ramon \& Gustavo E. Romero

A\&A, 503, 673-681, 2009

5. Gamma-Ray Variability from Wind Clumping in High-Mass X-Ray Binaries with Jets

Stanley P. Owocki; Gustavo E. Romero; Richard H.D. Townsend; Anabella T. Araudo

ApJ, 696, 690-693, 2009

6. Massive protostars as gamma-ray sources

Valentí Bosch-Ramon, Gustavo E. Romero, Anabella T. Araudo\& Josep M. Paredes

A\&A, 511, 1-10, 2010

7. High-Energy Emission from Jet-Cloud Interactions in AGNs

Anabella T. Araudo, Valentí Bosch-Ramon \& Gustavo E. Romero

IJMPD, 19, 931-936, 2010 
8. Gamma rays from cloud penetration at the base of AGN jets Anabella T. Araudo, Valentí Bosch-Ramon \& Gustavo E. Romero A\&A, en prensa, 2010 [arXiv:1007.2199]

- Publicaciones en revistas nacionales con referato

1. High-energy emission from Abell 3376

Anabella T. Araudo, Sofía A. Cora \& Gustavo E. Romero

BAAA 1 , 50, 303-306, 2007

2. Gamma-ray emission from jet-clump interactions

Gustavo E. Romero, Anabella T. Araudo, Stanley P. Owocki \& Richard Townsend

BAAA, 50, 319-322, 2007

3. Interactions of jets and clumpy stellar winds in high-mass microquasars

Anabella T. Araudo, Valentí Bosch-Ramon \& Gustavo E. Romero

BAAA, 51, 305-308, 2008

4. Jet-cloud interactions in the BLR of Centaurus $A$

Anabella T. Araudo, Valentí Bosch-Ramon \& Gustavo E. Romero

BAAA, 52, 255-258, 2009

- Publicaciones en actas de congresos

1. Gamma-ray emission from massive young stellar objects: the case of IRAS $16547-4247$

Anabella T. Araudo, Gustavo E. Romero, Valentí Bosch-Ramon \& Josep M. Paredes

Revista Mexicana de Astron. Serie de Congresos, 33, 159, 2008

2. Using gamma-rays to probe the clumped structure of stellar winds

Gustavo E. Romero, Stanley P. Owocki, Anabella T. Araudo, Richard Townsend \& Paula Benaglia

Clumping in hot-star winds, Potsdam: Univ.-Verl., 191-194, 2008

3. Non-thermal emission from massive YSOs. Exploring the spectrum at high energies

Anabella T. Araudo, Gustavo E. Romero, Valentí Bosch-Ramon \& Josep M. Paredes

First La Plata International School: Compact Objects and their Emission, I. Andruchow \& G.E. Romero, eds., (2008) [arXiv:0806.2306]

4. Gamma-radiation from the galaxy cluster Abell 3376

Sofía A. Cora, Anabella T. Araudo \& Gustavo E. Romero

American Institute of Physics, Conference Proceedings (AIPC), 1085, 573-576, 2008

\footnotetext{
${ }^{1}$ Boletín de la Asociación Argentina de Astronomía
} 
5. Jet-Cloud Interactions in AGNs

Anabella T. Araudo, Valentí Bosch-Ramon \& Gustavo E. Romero

2009. [arXiv:0908.0926]

6. High-energy flares from jet-clump interactions

Anabella T. Araudo, Valentí Bosch-Ramon \& Gustavo E. Romero

Astron. Soc. of the Pacific Conference Series, Eds.: Josep Martí, Pedro L. Luque-

Escamilla and Jorge A. Combi, 422, 32-40, 2010

7. Gamma-rays from massive protostars

Gustavo E. Romero, Anabella T. Araudo, Valentí Bosch-Ramon \& Josep M. Paredes

Astron. Soc. of the Pacific Conference Series, Eds.: Josep Martí, Pedro L. LuqueEscamilla and Jorge A. Combi, 422, 100-108, 2010

8. Gamma-Ray Variability from Stellar Wind Porosity in Microquasar Systems Stanley P. Owocki; Gustavo E. Romero; Richard H.D. Townsend; Anabella T. Araudo

Astron. Soc. of the Pacific Conference Series, Eds.: Josep Martí, Pedro L. LuqueEscamilla and Jorge A. Combi, 422, 49-54, 2010

- Publicaciones no relacionadas a la tesis

1. Extreme microvariability of blazars: fact and fiction

Anabella T. Araudo, Sergio A. Cellone \& Gustavo E. Romero

BAAA, 48, 379, 2005

2. Multifrecuency variability of the blazar AO 0235+164

The WEBT campaign in 2004-2005 and long-term SED analysis

C.M. Raiteri, M. Villata, M. Kadler et al.

A\&A, 459, 731, 2006

3. Extremely violent optical microvariability in blazars: fact or fiction?

Sergio A. Cellone, Gustavo E. Romero \& Anabella T. Araudo

MNRAS, 374, 357, 2007

4. Detection of nonthermal emission from the bow shock of a massive runaway star Paula Benaglia, Gustavo E. Romero, Josep Martí, Cintia S. Peri \& Anabella T. Araudo

A\&A Letters, en prensa (2010) [arXiv:1007.3279] 


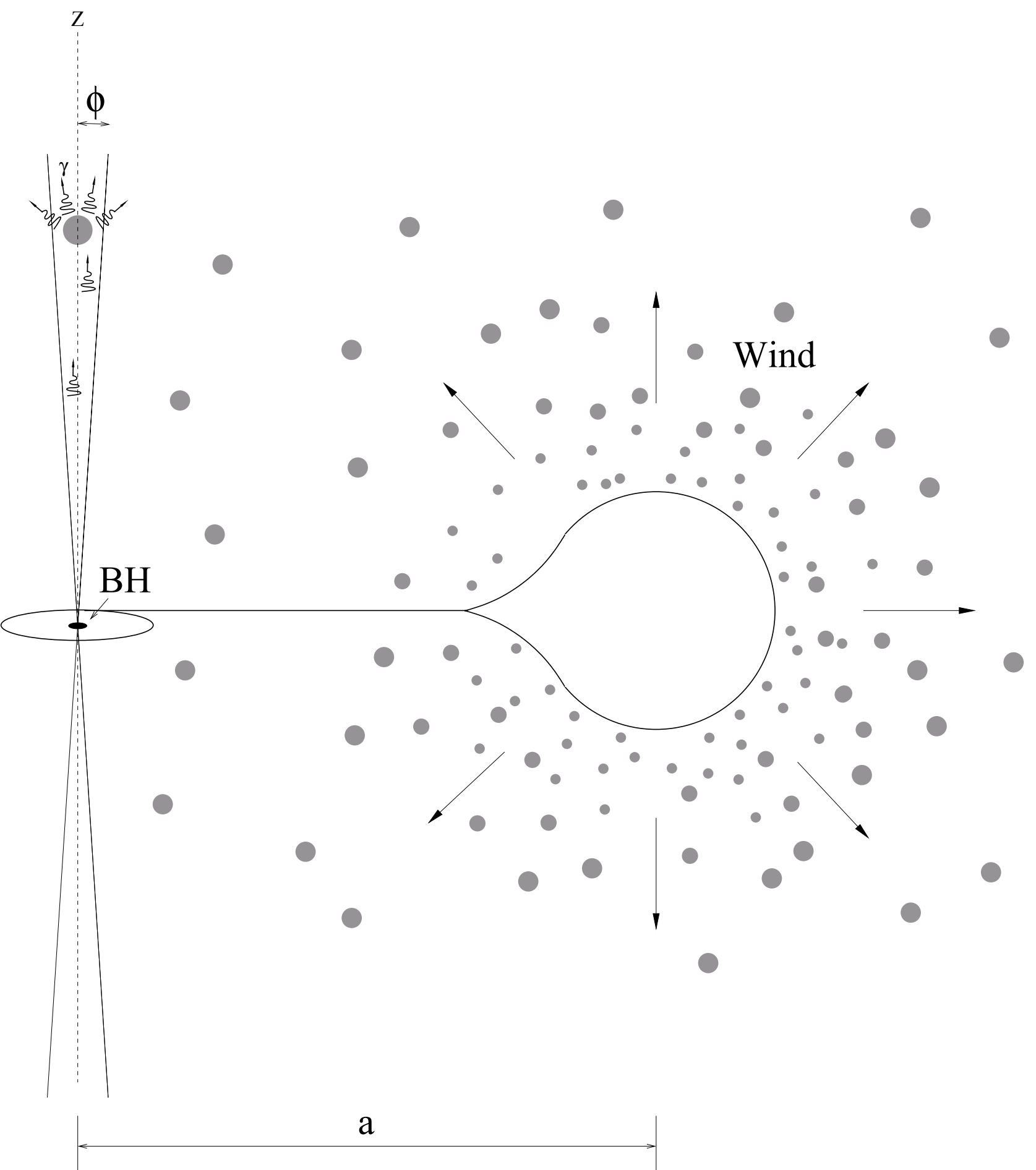




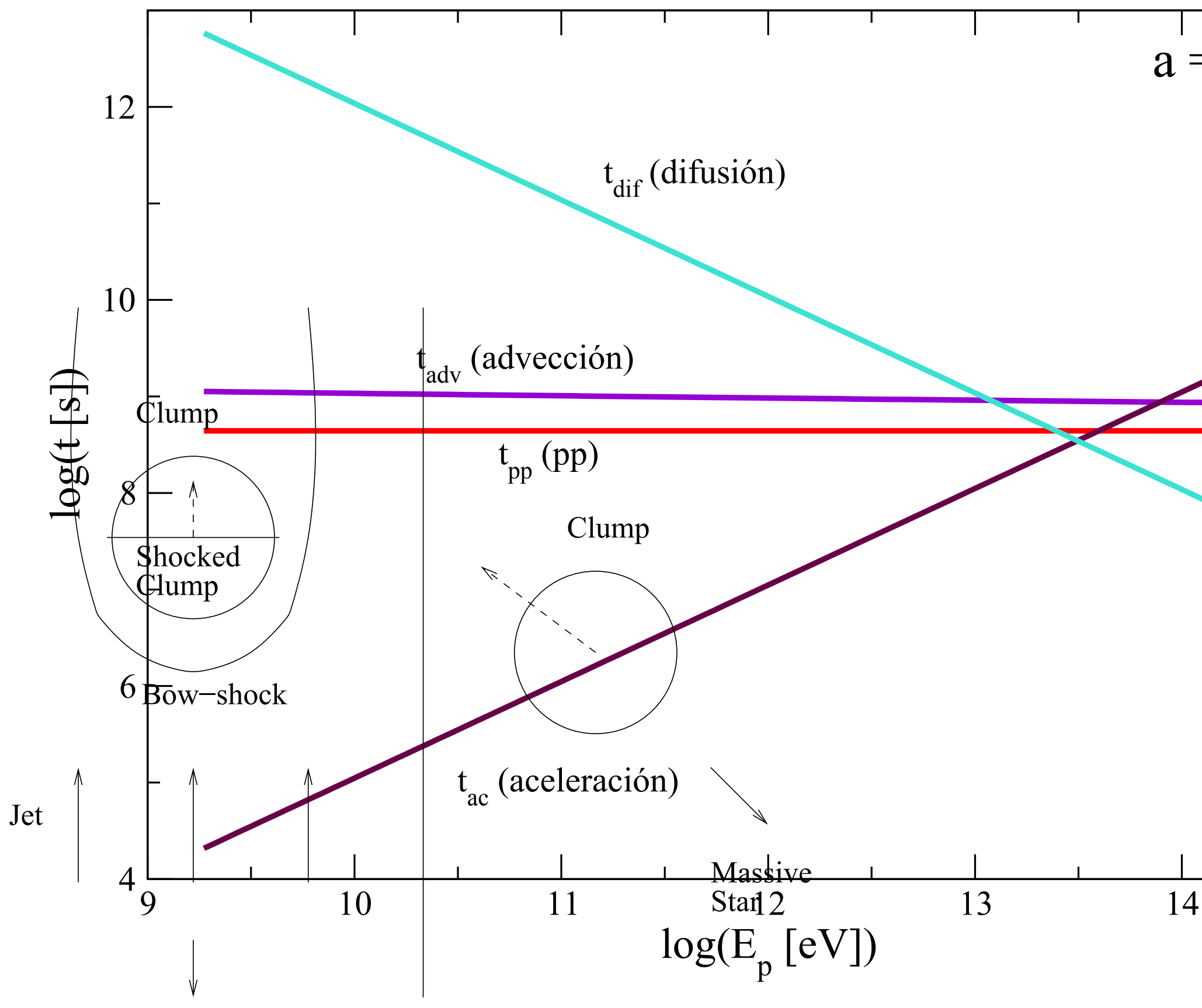

Compact Object 


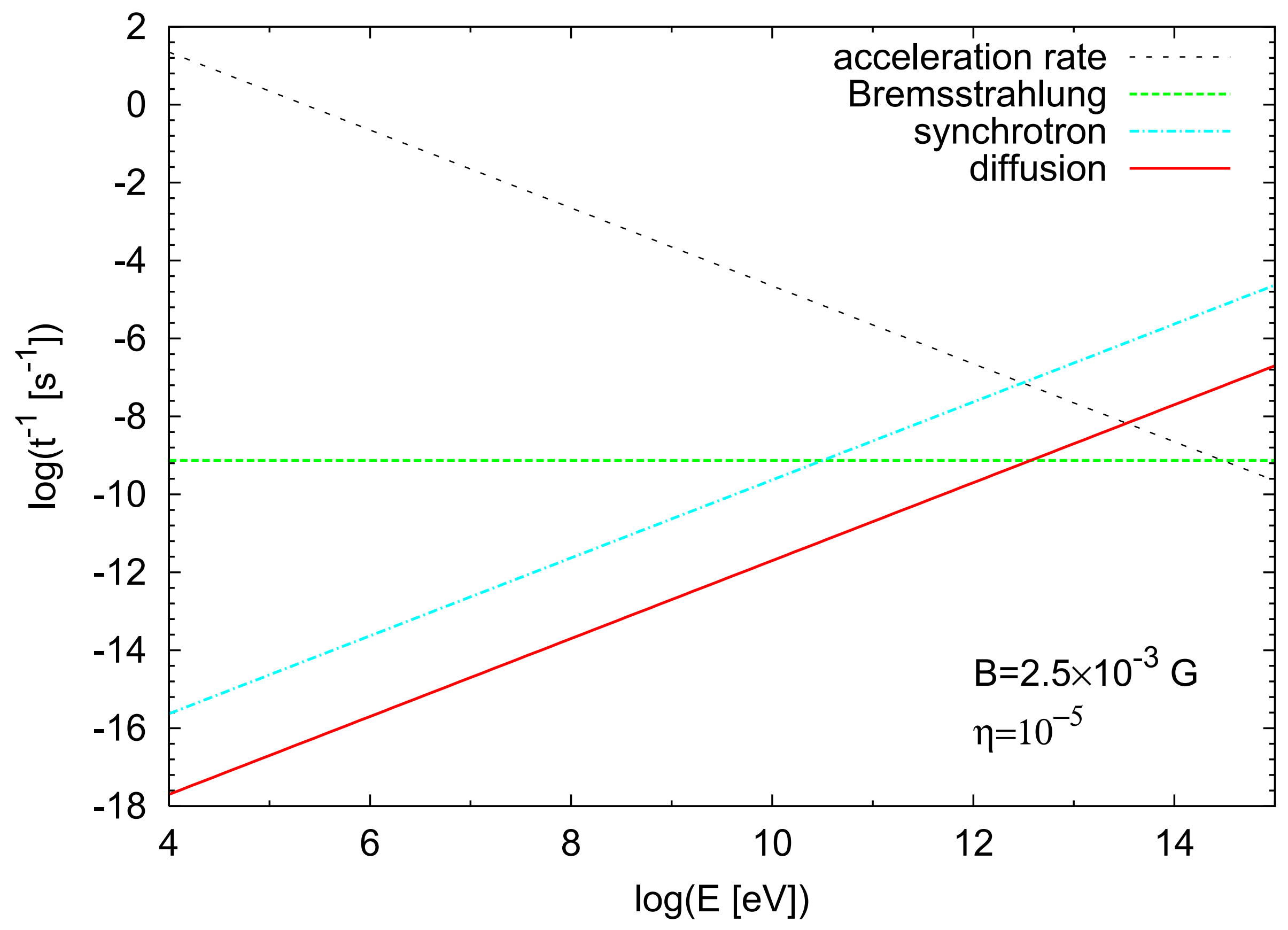

